\title{
DEVELOPMENT AND CHARACTERIZATION OF PHOSPHOLIPID ENCAPSULATED QUANTUM DOT CONSTRUCTS FOR BIOLOGIC APPLICATIONS
}

\author{
A Thesis Presented to the Faculty of \\ California Polytechnic State University, San Luis Obispo \\ In Partial Fulfillment of the Requirements for the Degree \\ Master of Science in Biomedical Engineering
}

By

Laura Christine Sparks

June 2012 
(C) 2012

Laura C. Sparks

ALL RIGHTS RESERVED 


\section{COMMITTEE MEMBERSHIP}

TITLE:

AUTHOR:

DATE SUBMITTED:

COMMITTEE CHAIR:

COMMITTEE MEMBER:

COMMITTEE MEMBER:
Development, Optimization, and

Characterization of Phospholipid Functionalized Quantum Dot

Constructs for Cell Targeting and Tissue Mapping

Laura Christine Sparks

June 22, 2012

Lily Laiho, $\mathrm{PhD}$

Richard Savage, $\mathrm{PhD}$

Rafael Jiménez-Flores, PhD 


\begin{abstract}
Laura Christine Sparks

DEVELOPMENT AND CHARACTERIZATION OF PHOSPHOLIPID ENCAPSULATED QUANTUM DOT CONSTRUCTS FOR BIOLOGIC APPLICATIONS
\end{abstract}

The American Cancer Society predicts that 577,190 cancer-related deaths and 1,638,910 newly diagnosed cases of cancer will occur in 2012. As these statistics show, cancer is a prevalent and devastating health issue; determined by the Mayo Clinic to be the second leading cause of death in the United States. Skin cancer is the most common form of cancer in the United States. In 2012 more than 68,000 Americans will be diagnosed with melanoma, 48,000 will be diagnosed with an early form of the disease that has not yet reached the lower levels of the epidermis, and more than 2 million people will be treated for basal cell or squamous cell skin cancer. Early and accurate detection is the most reliable way to ensure a positive outcome and the ultimate survival of the patient. As the most aggressive form of skin cancer, survival of melanoma is especially connected to early detection. Current methods for the initial detection of potential cancerous masses and lesions rely on visual examination, palpitation, and biopsy. Accurate determination of the presence of cancerous cells in a biopsy is especially difficult at the early stages when only a small percentage of cells in the biopsied mass show the morphological traits associated with being cancerous. This circumstance often results in a false negative (FN), delaying the necessary treatment until the cancer has reached a more developed stage. Developing more accurate methods for the detection of cancerous cells within a biopsy would aid in alleviating this problem. An improvement to the conventional method of visually examining biopsied tissues for the presence of cells with abnormal morphologies can be offered by utilizing the model of functionalized quantum dot (QD) constructs. 
Quantum dots are nano-particles composed of semi-conducting materials that fluoresce at discrete wavelengths when irradiated by a high energy UV source. QD constructs are cadmiumselenium/zinc-sulfide $(\mathrm{CdSe} / \mathrm{ZnS})$ quantum dots encapsulated within a bovine derived milk phospholipid micelle. QD constructs provide a potential mechanism for the identification of cancerous cells within a biopsy. Appreciating the scope of the clinical problem and understanding the potential of QDs, the objective of this thesis is to develop a primary model for the solubilization, encapsulation, and primary phospholipid functionalization of two distinct sizes of CdSe/ZnS QDs. The first stage of this thesis optimized the currently utilized protocol for synthesizing cadmium-selenium (CdSe) quantum dots to develop a set of parameters for consistently producing white fluorescing CdSe cores (WFCs) and CdSe/ZnS QDs of 505nm and $555 \mathrm{~nm}(+/-10 \mathrm{~nm})$. The application of synthesis times, temperatures, and quenching methods were employed to achieve this. The second stage developed a phospholipid encapsulation method for the initial functionalization and suspension of the hydrophobic QDs in aqueous media via encapsulation within phospholipid micelles. The final stage of this thesis focused on the successful introduction of the QD constructs into keratinocyte cells. Calcein and Ethidium homodimer-1 stains were applied to determine cell viability, Histochoice was applied as a fixative, and Hoechst staining was employed for cell nuclei identification. Analysis using confocal microscopy suggests successful attachment of QD constructs, in $0.1 \%$ w/v keratinocyte media, to the exterior of keratinocyte cell membranes with a 30\% average cell survival rate at 24 hours after sample introduction. Future research investigating the interaction of QD constructs with biologic mediums of greater physiological complexity, as well as application of a secondary functionalization, are the next steps on the path toward achieving a viable mechanism for targeting and identifying cancerous cells within a biopsy. 


\section{Acknowledgements}

I would like to thank Dr. Lily Laiho for her support both on and off the thesis field. Your work ethic, positive attitude, and seemingly endless energy are an inspiration. I value all of the conversations we have had, whether they were related to my thesis, introducing me to the wonder that is Grammar Girl, giving me objective and honest assessments throughout my search for employment, helping me to believe that lack of job experience doesn't equate to lack of merit, or simply sharing your views on some of the random idiosyncrasies of life. I sincerely thank you. I would also like to thank Dr. Savage, both as an advisor and professor. You made materials engineering an interesting and favorite subject; in my next life I plan on majoring in it. I especially appreciate your willingness to humor me and listen to my constant ramblings about lab organization. Dr. Jimenez, your excitement for trying something novel has been contagious. You never failed to provide support and words of encouragement, both for this scholastic life and the next, affording me a fun experience I will always treasure.

Many of my peers contributed their time, knowledge, and skills helping me to set up and prepare integral components of this thesis, I would like to particularly thank Carl Dargitz, the keratinocyte cell keeper and master culture custodian with unending patience, Davis Carlin, Patrick McBride, Josh Angell, and Susan Harada for their conversations and tutorials, and Andrea Lambausch for always providing me with phospholipids, fresh cream, and tails of sheep keeping shenanigans.

The continued support of my physics family over the last two years has been tremendous; the carnivals, brunches, baby showers, birthday parties, and department functions have all made me feel so very fortunate to be a part of such a tremendous group of people. Alison and Robin, from both near and far, you have been with me, filling my journey with laughter, sharp 
exclamations, truth, and absolute joy; Vi amo tanto. Roger and the Road Runners of SLO, you gave me a much needed outlet and a damn fun group of people, making the miles fly by and the ice baths completely worth it. Kari, my oldest and dearest friend, my sister in all ways but one, I thank you for everything that you have been for me; you always bring me back to my center. Jaime, forever my partner in conscientious objecting and wine drinking, thank you for giving me so many great escapes; Live Long and Prosper friend. My guys and gals in La Verne, you are the epitome of what friends and love and family should be, you keep me grounded and I am forever grateful that I have always had you to come home to. Mom and Dad, you are my rock, my soul mates, my shining example of what hard work, dedication, honor, and a deep soul can accomplish. I thank you endlessly. 


\section{TABLE OF CONTENTS}

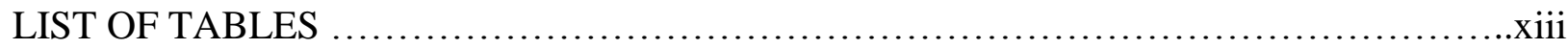

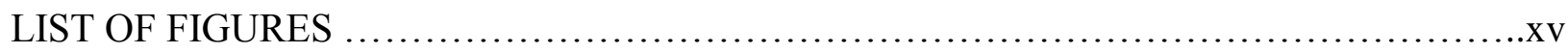

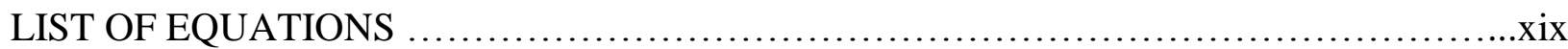

CHAPTER 1 - INTRODUCTION .......................................................

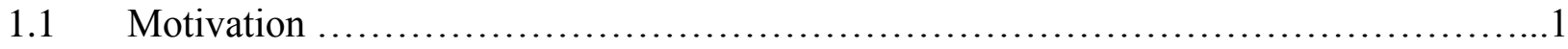

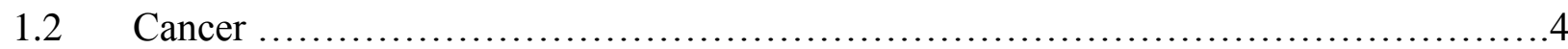

1.2.1 Diagnosis of Skin Cancer ................................................. 6

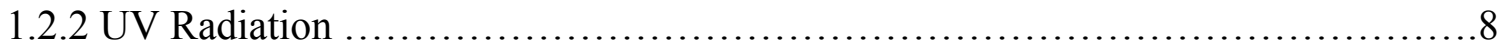

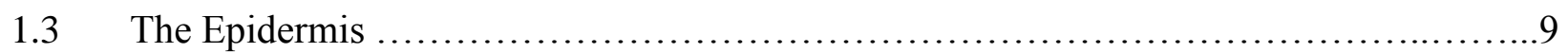

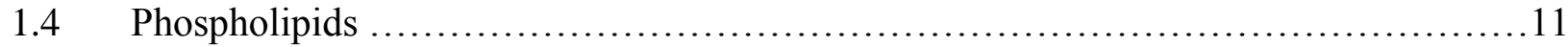

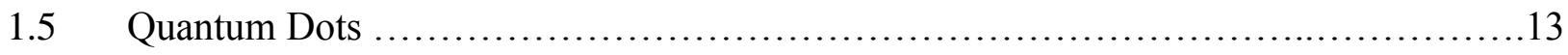

1.5.1 Quantum Confinement and the Bohr Exciton Radius ..........................18

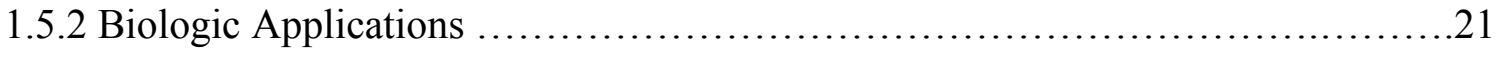

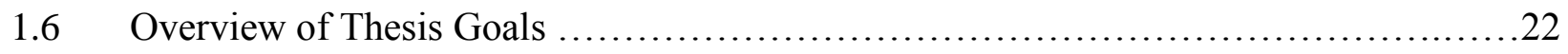

CHAPTER 2 - DEVELOPMENT AND EVALUATION OF CdSe/ZnS QUANTUM DOTS ..24

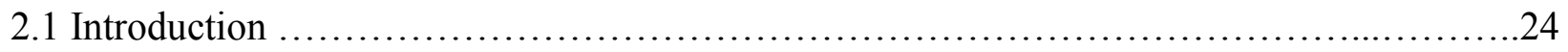

2.2 Method for CdSe Core and ZnS Quantum Dot Synthesis ...............................27

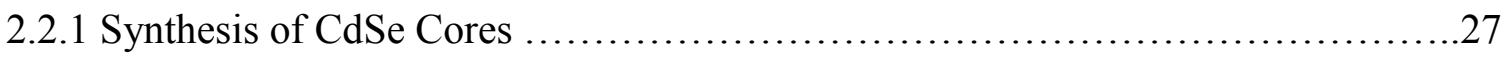

2.2.1.1 Experimental Variables Investigated for the General Synthesis of CdSe

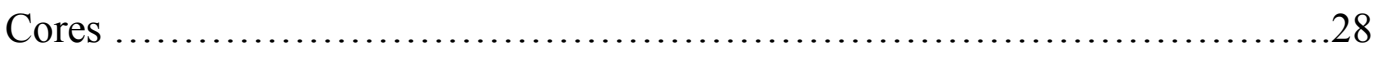

2.2.1.2 Synthesis of White Fluorescing CdSe Cores (WFCs) ..................29

2.2.2 Synthesis of ZnS Coated Quantum Dots ....................................30 


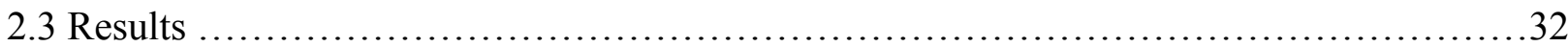

2.3.1 Synthesis of CdSe Cores ........................................................

2.3.1.1 Experimental Variables Investigated for the General Synthesis of CdSe

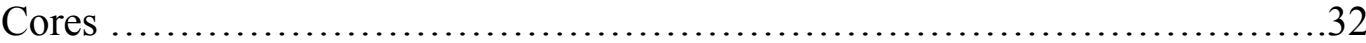

2.3.1.2 Temporal Effects on CMW, Intensity, and FWHM of CdSe Cores ......34

2.3.1.3 Temporal Wavelength Shifts in White Fluorescing CdSe Cores .........39

2.3.2 Synthesis of ZnS Coated Quantum Dots....................................42

2.3.2.1 ZnS Coating Synthesis Temperature Variation ......................42

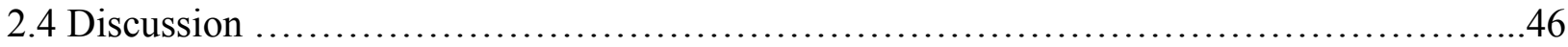

2.4.1 Synthesis of CdSe Cores ...............................................46

2.4.1.1 Experimental Variables Investigated for the General Synthesis of CdSe

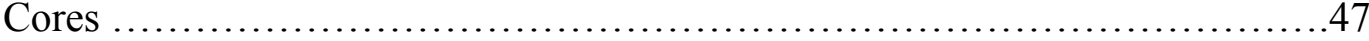

2.4.1.2 Synthesis of White Fluorescing CdSe Cores .........................48

2.4.1.3 Temporal Wavelength Shifts in White Fluorescing CdSe Cores .........48

2.4.2 Synthesis of ZnS Coated Quantum Dots ....................................48

2.4.2.1 ZnS Coating Synthesis Temperature Variation .......................49

CHAPTER 3 - QUANTUM DOT CONSTRUCT SYNTHESIS .............................50

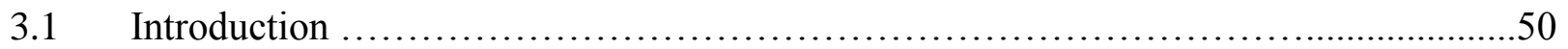

3.2 Method for Solubilization and Phospholipid Encapsulation of CdSe/ZnS QDs .........50

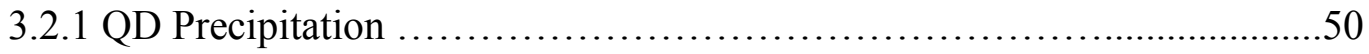

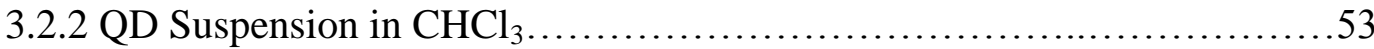


3.2.3 Phospholipid Concentration, Suspension, and Encapsulation .53

3.2.3.1 Experimental Trials - Round One and Two ..................53

3.2.3.2 Final Encapsulation Method ..............................58

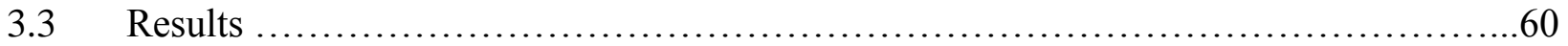

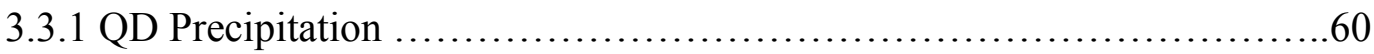

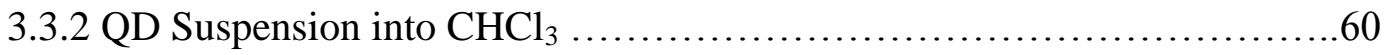

3.3.3 Phospholipid Concentration, Suspension, and Encapsulation ............61

3.3.3.1 Experimental Trials - Round One and Two .......................61

3.3.3.2 Final Encapsulation Method ..............................71

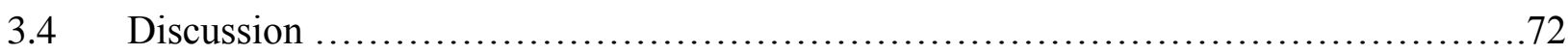

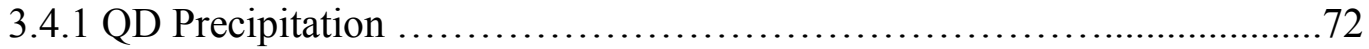

3.4.2 Phospholipid Concentration, Suspension, and Encapsulation .............73

3.4.2.1 Experimental Trials - Round One and Two .................73

3.4.2.2 Final Encapsulation Method ............................. 75

CHAPTER 4 - INTRODUCING QUANTUM DOT CONSTRUCTS INTO A

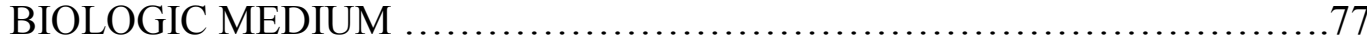

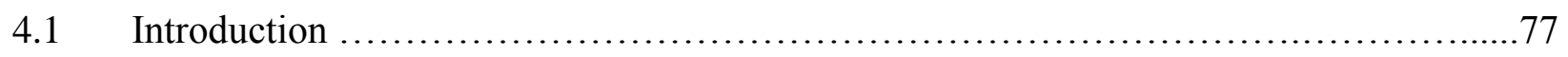

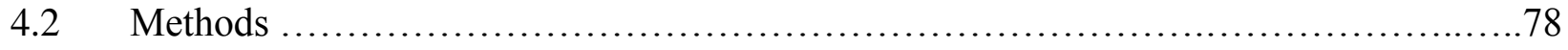

4.2.1 QD Construct Introduction into Keratinocytes Cell Cultures ..............78

4.2.1.1 QD Construct Control and Test Solutions - R1 ................79

4.2.1.2 QD Construct Control and Test Solutions - R2 ...............83

4.2.1.3 Duration of Keratinocyte Trial - R1 .....................86

4.2.1.4 Duration of Keratinocyte Trial - R2 ......................87

4.2.2 Implementation of White Light Optical Imaging $\ldots \ldots \ldots \ldots \ldots \ldots \ldots \ldots . . . .68$ 
4.2.2.1 Optical Imaging - R1 ...................................90

4.2.2.2 Optical Imaging - R2 .................................. 91

4.2.3 Fluorescence Staining ............................................. 92

4.2.3.1 Calcein/EthD-1 Live/Dead Cell Viability Stain - R1and R2 _....94

4.2.3.2 Histochoice/Hoechst Nucleus Stain - R2 .....................95

4.2.4 Confocal and DIC Imaging ....................................... 96

4.2.4.1 Keratinocyte Cell Viability - R1 ..............................96

4.2.4.2 Keratinocyte Cell Viability - R2 ….......................97

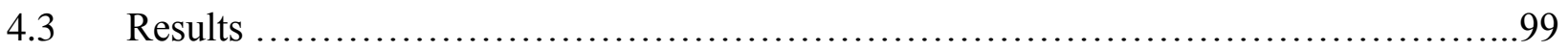

4.3.1 QD Construct Introduction into Keratinocyte Cell Cultures ...............99

4.3.1.1 QD Construct Control and Test Solutions - R1 .................99

4.3.1.2 QD Construct Control and Test Solutions - R2 ..............102

4.3.1.3 Optical Imaging - R1 ....................................103

4.3.1.4 Keratinocyte Cell Viability and Cell-QD Construct Interaction - R1 ......................................105

4.3.1.5 Optical Imaging - R2 ...................................113

4.3.1.6 Keratinocyte Cell Viability and Cell-QD Construct

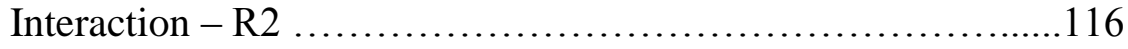

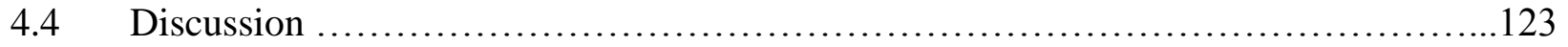

4.4.1 QD Construct Introduction into Keratinocyte Cell Cultures ..............123

4.4.1.1 QD Construct Control and Test Solutions - R1 ...............123

4.4.1.2 QD Construct Control and Test Solutions - R2 ..............124

4.4.1.3 Optical Imaging - R1 .....................................125

4.4.1.4 Optical Imaging - R2 ...................................126 
4.4.1.5 Keratinocyte Cell Viability and Cell-QD Construct Interaction - R1.

4.4.1.6 Keratinocyte Cell Viability and Cell-QD Construct Interaction $-\mathrm{R} 2$

CHAPTER 5 - DISCUSSION AND CONCLUSIONS ................................. 130

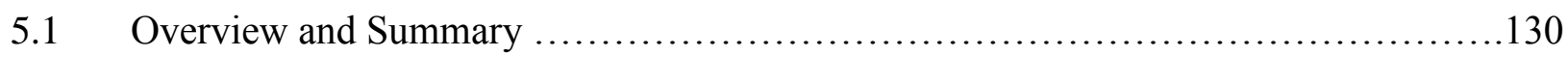

5.2 Challenges, Limitations, and Future Work .......................................

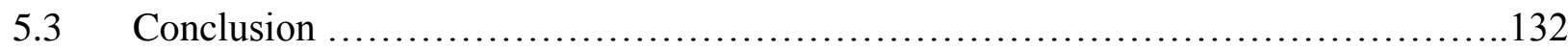

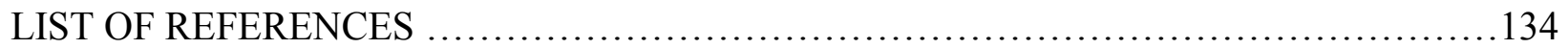

APPENDIX A - ABBREVIATIONS …...............................................143

APPENDIX B - PROTOCOLS AND EXPERIMENTAL DETAILS..........................144

Appendix B.1 Cal Poly Standard CdSe/ZnS Synthesis Protocol and Fluorescence

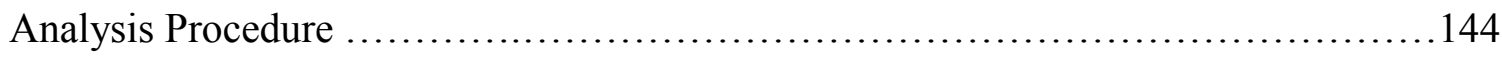

Appendix B.2 Absorbance Measurement Procedure ...................................164

Appendix B.3 Wavelength Specific CdSe and CdSe/Zn Synthesis ....................168

Appendix B.4 Precipitation and Resuspension of CdSe/ZnS Quantum Dots ............171

Appendix B.5 Quantum Yield Calculation......................................173

Appendix B.6 Solubilization and Phospholipid Encapsulation of CdSe/ZnS Quantum

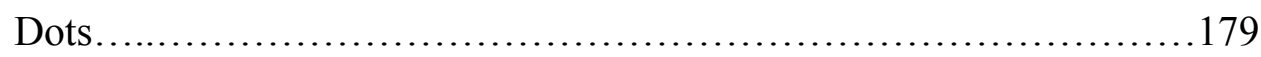

Appendix B.7 Introduction of QD Construct Test Solutions to Keratinocyte Cell

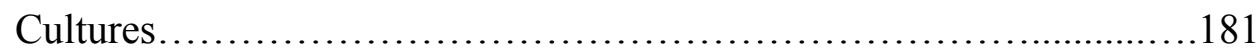

Appendix B.8 Fluorescent Staining - Histochoice and Hoechst .......................184

Appendix B.9 Fluorescent Staining - Calcein EthD-1 ............................186 


\section{LIST OF TABLES}

Table 2.1 Effects of Temperature on CMW, Intensity, and FWHM for 30, 60, and 90s Samples

Cooled in Air

Table 2.2 Two Sample t-test of Effects of Temperature on CMW, Intensity, and FWHM for

Each Reaction Time Point................................................... 34

Table 2.3 Averages of, and Standard Deviations for, the CMW, Intensity, and FWHM of CdSe samples for 30, 60, and 90s Reaction Times. Left: averages. Right: standard deviations .35

Table 2.4 Single Factor ANOVA Test p-values for CMW, Intensity, and FWHM of CdSe

Cores at Reaction Times of 30, 60, and 90s.

Table 2.5 Two Sample t-test p-values Corresponding to the Effects of Quenching in

Acetone vs. Air for Three Sets of CdSe Core Synthesis Reactions

Table 2.6 CMW, Intensity, and FWHM Values for WFCs Coated at $70^{\circ} \mathrm{C}$ and $160^{\circ} \mathrm{C}$ 43

Table 2.7 Averages and Standard Deviations of CMW, Intensities, and FWHMS of WFCs

Coated at $70^{\circ} \mathrm{C}$ and $160^{\circ} \mathrm{C}$ 43

Table 3.1 Concentration and Ratio Test Parameters Applied with 2.5mg/mL Trial ............54

Table 3.2 PL Encapsulation and Evaporation Parameters..................................55

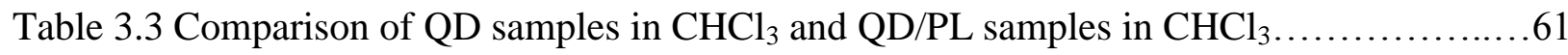

Table 4.1 Summary of Round One QD Construct Control and Test Solutions ................81

Table 4.2 Summary of Round Two QD Construct Control and Test Solutions..................84

Table 4.3 Keratinocyte Trial Time Points in Round Two.................................88

Table 4.4 Progression of Changes in Fluorescence Spectrum During Synthesis of QD Construct Solution 42612wEN.2a. 100 
Table B.5.1 Concentration and Dilution Calculation Table .175

Table B.5.2 Sample Data for Calculating the Area Under a Fluorescence Spectral Peak ......176 


\section{LIST OF FIGURES}

Figure 1.1 The eight stages of tumor growth and expansion leading up to metastasis.............5

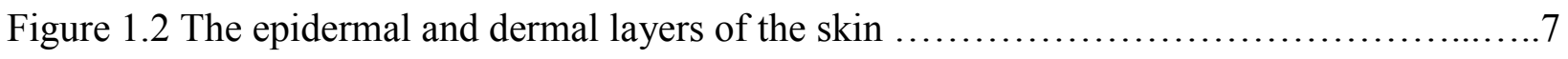

Figure 1.3 The electromagnetic spectrum.............................................

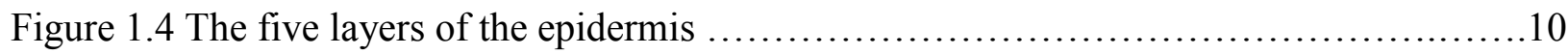

Figure 1.5 Phospholipid molecular structure and aqueous aggregation tendencies..............11

Figure 1.6 Phospholipid fatty acid chain attachments to QD surface........................13

Figure 1.7 Bands gaps of conduction and valence bands...............................

Figure 1.8 Atomic orbital electron lowest energy configuration for silicon..................15

Figure 1.9 Discrete energy levels to continuous energy bands $\ldots \ldots \ldots \ldots \ldots \ldots \ldots \ldots \ldots \ldots \ldots 17$

Figure 1.10 QD size, Bohr exciton radius, band gap and atomic energy levels.................19

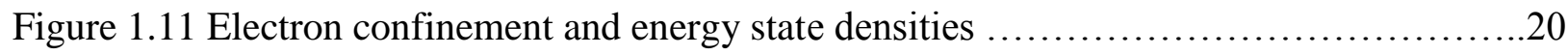

Figure 1.12 QD size, band gap energy and photon emission wavelength relationship...........21

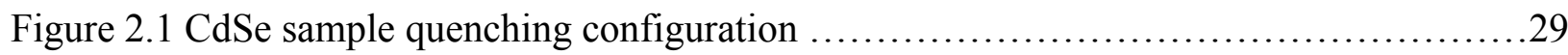

Figure $2.2 \mathrm{CdSe}$ core sample division for $\mathrm{ZnS}$ shelling temperature tests...................32

Figure 2.3 Comparison of reaction temperature on CdSe core synthesis ...........................33

Figure 2.4 Comparison of reaction time on CdSe core synthesis ..............................

Figure 2.5 Fluorescence spectrum of CdSe core solutions for 30, 60, amd 90s reaction times...36

Figure 2.6 Digital image of 0 and 60s averaged CdSe core sample sets under UV............37

Figure 2.7 Fluorescence spectrums of 0 and 60 s averaged CdSe core sample sets .............38

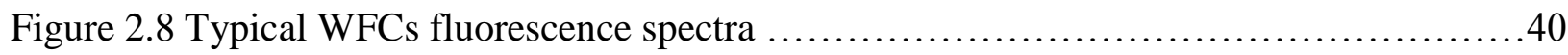

Figure 2.9 Fluorescence spectra of temporal shift of WFCs day $1 \ldots \ldots \ldots \ldots \ldots \ldots \ldots \ldots \ldots \ldots \ldots$

Figure 2.10 Fluorescence spectra of temporal shift of WFCs day $23 \ldots \ldots \ldots \ldots \ldots \ldots \ldots \ldots \ldots \ldots \ldots$ 
Figure 2.11 Fluorescence spectra of temporal shift of WFCs day 1 to day $23 \ldots \ldots \ldots \ldots \ldots \ldots 42$

Figure 2.12 Comparison of $70^{\circ} \mathrm{C}$ and $160^{\circ} \mathrm{C} \mathrm{ZnS}$ shelling temperatures .....................44

Figure 2.13 Qualitative comparison of $\mathrm{ZnS}$ shelling temperatures.......................45

Figure 2.14 Averaged fluorescence spectrum of white fluorescing starting CdSe cores ........45

Figure 2.15 Averaged fluorescence spectrum of green fluorescing starting CdSe cores ........46

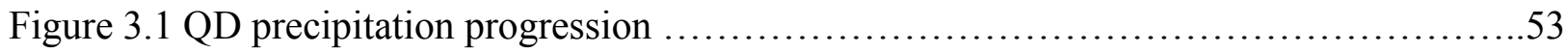

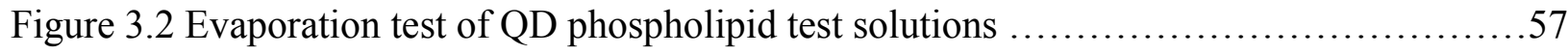

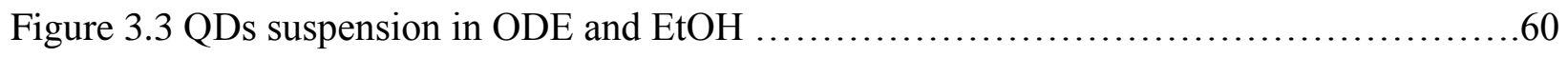

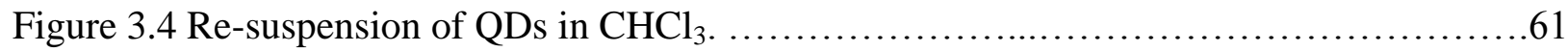

Figure 3.5 Phospholipid test solution fluorescence scattering ............................62

Figure 3.6 Fluorescence spectra of phospholipids test solutions $-100 \mathrm{~ms}$ integration...........63

Figure 3.7 Fluorescence spectra of phospholipids test solutions $-500 \mathrm{~ms}$ integration...........63

Figure 3.8 Fluorescence spectra of the filtered encapsulation test solutions ..................64

Figure 3.9 Image series QD: phospholipid ratio trials in suspension ......................65

Figure 3.10 Image series QD: phospholipid ratio trials pre-evaporation......................65

Figure 3.11 Image series QD: phospholipid ratio trials post-evaporation....................66

Figure 3.12 Confocal image QD phospholipid control test solutions........................67

Figure 3.13 Confocal image of 2.5:0.5mL QD phospholipid test solution ....................67

Figure 3.14 Confocal image of 2.5:1.0mL QD phospholipid test solution ....................68

Figure 3.15 Confocal image of 3.5:0.5mL QD phospholipid test solution ....................68

Figure 3.16 Encapsulation progression of test trial set D............................. 70

Figure 3.17 Encapsulation progression of test trial sets A, B, C, and D ...................70

Figure 3.18 Trial B2 encapsulation solution...................................... 70 
Figure 3.19 QD phospholipid mixture pre-evaporation................................ 71

Figure 3.20 Change in QD construct solution viscosity during synthesis.....................72

Figure 4.1 Four-well slide configurations. ....................................... 80

Figure 4.2 Fluoresence progression of $\mathrm{CdSe} / \mathrm{ZnS}$ sample $42612 \mathrm{wQD} .2 \mathrm{a} \ldots \ldots \ldots \ldots \ldots \ldots \ldots \ldots . \ldots 3$

Figure 4.3 Fluorescence progression of $\mathrm{CdSe} / \mathrm{ZnS}$ sample 42612 oEN.1a ....................84

Figure 4.4 Neonatal foreskin keratinocytes (NFK) colonization...........................89

Figure 4.5 Sequence of Cell Culture - Test Solution Interaction and Imaging ................91

Figure 4.6 Excitation emission spectra of Calcein (495/515) and EthD-1 (495/635).........93

Figure 4.7 Excitation emission spectra of Hoechst 34580 stain..........................94

Figure 4.8 Adaptation of four-well with thick specimen slide base $\ldots \ldots \ldots \ldots \ldots \ldots \ldots \ldots \ldots \ldots 9$

Figure 4.9 Fluorescence spectrum of CdSe combination core sample (42612wCMBO.2) .....100

Figure 4.10 Fluorescence spectrum of QD construct synthesis solution progression............101

Figure 4.11 Fluorescence spectrum: Effects of phospholipid concentration.................101

Figure 4.12 QD construct test solutions ....................................... 102

Figure 4.13 Fluorescence spectra of QD construct test solutions .........................103

Figure 4.14 Optical image series of R1 well a1.1 (fresh media control)....................104

Figure 4.15 Optical image series of R1 well b1.3 (keratinocyte media test).................105

Figure 4.16 Confocal image series of well b1.3 (processed krt media control)................106

Figure 4.17 Confocal image series of well b1.1 (fresh krt media control) ...................106

Figure 4.18 Image series of well b2.6 (PL krt filtered) pre-introduction of test solutions ......107

Figure 4.19 Image series of well b2.7 (PL krt NF) pre-introduction of test solutions..............108

Figure 4.20 Image series of well b3.10 (QD PL krt filtered) pre-introduction of test solutions.109

Figure 4.21 Digital image of well b3.10 as seen with wide-field fluorescence laser ...........110 
Figure 4.22 Confocal image of a seemingly burst cell in well b3.10 (QD PL krt filtered) .....110

Figure 4.23 Series of L/D filter confocal images of well a3.9 (QD PL krt filtered)............111

Figure 4.24 Digital image of well a3.9, representative of the series A1-4 in figure $4.23 \ldots . . .111$

Figure 4.25 Image series of b3.12 (QD PL krt NF) pre-introduction of test solutions............112

Figure 4.26 Multiple focal plane confocal images of well a3.11 (QD PL krt NF) ............113

Figure 4.27 Digital image of well a3.12 (QD PL krt NF) under wide-field fluorescence........113

Figure $4.28 \mathrm{R} 2$ cell culture pre-introduction of the test solutions..........................115

Figure 4.29 R2 cell culture post-introduction of the test solutions.........................116

Figure 4.30 Confocal image of keratinocyte control with L/D and DIC dilters ..............117

Figure 4.31 Comparison of control versus QD-construct test solution for QD signature.........118

Figure 4.32 Series of confocal images taken of R2 b1.4 (QD construct B 0.2 micron filter)...118

Figure 4.33 Series of DAPI QD filter images of R2 b2.5 (QD construct B 0.45 micron filter)..119

Figure 4.34 Series of DAPI QD filter images of R2 b2.6 (keratinocyte control media)..........120

Figure 4.35 Series of DAPI QD filter images of R2 b2.7 (QD construct B 0.45 micron filter)..120

Figure 4.36 Series of confocal filter images of R2 c2.5 (QD construct B 0.45 micron filter)...121

Figure 4.37 Series of confocal filter images of R2 c2.6, 7, 8 QD identification ..............122

Figure 4.38 Series of DAPI QD filter images of R2 c1.1 (keratinocyte control media)..........123

Figure 4.39 Digital image of well b3.10 (QD PL krt filtered) under wide-field fluorescence ...125

Figure B.5.1 Corresponding O.D. and wavelength values on an absorbance spectrum.........176 


\section{LIST OF EQUATIONS}

$$
Q=Q_{R}\left(\frac{m}{m_{R}}\right)\left(\frac{n^{2}}{n_{R}^{2}}\right)
$$

$$
Q=Q_{R}\left(\frac{I}{I_{R}}\right)\left(\frac{O D_{R}}{O D}\right)\left(\frac{n^{2}}{n_{R}^{2}}\right)
$$

Equation B.5.1 ............................177

Equation B.5.2 ….......................178 


\section{Chapter 1 - Introduction}

\subsection{Motivation}

According to the 2012 cancer statistics conducted by the American Cancer Society it is expected that $1,638,910$ new cases of cancer will be diagnosed in 2012 (1). Additionally it is expected that 577,190 deaths will occur within the diagnosed population currently living with cancer (1). As these statistics show, cancer is a prevalent and devastating health issue and the second leading cause of death in the United States (2). Cancerous tumors can grow quickly, increasing their rate of cell proliferation, and metastasize to other areas of the body. If gone undetected, until the later stages of development, it is often too late for the patient to survive (3). The most common type of cancer found in the United States is skin cancer (4). In 2012 more than 68,000 Americans will be diagnosed with melanoma, 48,000 will be diagnosed with an early form of the disease that has not yet reached the lower levels of the epidermis, and more than 2 million people will be treated for basal cell or squamous cell skin cancer (4).

Early and accurate detection is the most reliable way to ensure a positive outcome and the ultimate survival of the patient (5). As the most aggressive form of skin cancer, once metastasis has occurred, survival of melanoma is especially connected to early detection (6). Current methods for the initial detection of potential cancerous masses and lesions rely on visual examination, palpitation, and for the most prevalent forms of cancer, annual screenings (5). Once the presence of cancerous tissue has been ascertained by a physician a biopsy is performed for confirmation. Initial confirmation of cancerous cells is based on visual inspection of the cells under a microscope by a pathologist (7). In cases where an initial assessment is used as the basis for dictating which treatment options will be followed, it is important that the evaluation of the biopsy be accurate. Accurate determination of the presence of cancerous cells in a biopsy is especially difficult at the 
early stages when only a small percentage of cells in the biopsied mass show the morphological traits associated with being cancerous. This circumstance often results in a false negative (FN), delaying the necessary treatment until the cancer has reached a more developed stage (8).

This propensity for FNs in the early stages of cancer development indicates there is a need for an ulterior method of identification that would recognize even a single cancerous cell. An improvement to the conventional method of visually examining biopsied tissues for the presence of cells with abnormal morphologies can be offered by utilizing the model of functionalized quantum dot (QD) constructs. QD constructs provide a mechanism in which cancerous cells present within a biopsy sample could potentially be detected.

Quantum dots made soluble and functionalized to target enzymes associated with specific cellular receptors are currently being used as replacements for organic fluorphores in biologic applications of cellular tagging and tissue mapping (9-13). Applying this same methodology toward targeting enzymes that are associated with the initial stages of growth and proliferation specific to cancerous cells provides a platform for detecting the onset of cancer at the initial phase of cellular manifestation; developing the capability to detect the presence of cancerous cells at their earliest stages of development, before the tumor reaches the stage of metastization, would greatly increase patient outcome and survival. In the last decade there have been several approaches for targeting cancer cells utilizing QDs. One such approach explored by Gao et al. in 2004 involved the encapsulation of QDs with an amphiphilic tri-block polymer and multifunctionalization of the encapsulated QDs with a prostate-specific membrane antigen (PSMA), a cell surface marker associated with prostate epithelial cells (9). In the Gao approach anti-body conjugation of the polymer encapsulated QDs was used for in vivo active tumor targeting. In 2008 an investigation into the creation and use of proximity activated nanoparticles utilizing a QD base and a fluorescently labeled cleavable peptide substrate modeled in an in vitro environment was 
conducted by Smith et al. (10). Smith's group synthesized carboxylated CdSe/ZnS QDs bioconjugated with a cleavable peptide substrate specific to matrix metalloproteases (MMPs). The goal of each of these methods was to develop a method for cancer targeting, drug delivery, and imaging using QDs.

The purpose of this thesis was to develop a base model for the solubilization, encapsulation, and primary functionalization of QDs using phospholipids as both the encapsulation and functionalization component. The methods for phospholipid encapsulation of QDs described in the following chapters were developed using the phospholipid encapsulation methods outlined by Fan and Carion in their 2002 and 2007 papers detailing the use of such constructs in applications of in vivo imaging. It is proposed that the basic method for the phospholipid encapsulation of QDs described in this thesis can be further modified to target additional cellular components through a secondary functionalization. These secondary functionalized QD constructs would provide a useful method for targeting and imaging cells of interest for future in vitro and in vivo studies. Further, secondary functionalization could then be fine-tuned to effectively create cell specific targeting constructs with the potential for use as an efficient and consistent mechanism for seeking out and confirming the presence of cancerous cells in in vitro studies.

This introduction will begin by presenting a fundamental overview of the physiology and stages of the development of cancerous cells, focusing specifically on melanoma. General components of the characteristic properties of UV radiation, as well as particular aspects pertaining to the effects of UV radiation on epidermal tissue, will also be discussed as well as a brief overview of epidermal physiology. Additionally, the chemical properties and biological interactions of phospholipids, and the physics related to the behavior of quantum dot will be provided. Understanding the chemical and biological interactions characteristic of phospholipids will give meaningful insight into the purpose for each step of the QD construct development process. 
Although a detailed knowledge of the physics behind the behavior of quantum dots is not essential for their creation, it is, I believe, a valuable and thoroughly interesting aspect of the process and therefore should not be neglected for the sake of brevity. Finally, in this introduction a concise summary of some of the alternative biologic applications for which QDs are currently being used will be provided in order to give a proper appreciation for the diverse and complex environment into which this thesis attempts to delve.

\subsection{Cancer}

Tumor is the general term used to describe a number of diseases that are characterized by the rapid and uncontrolled division of mutated cells. The source of this abnormal growth of body tissue is called the neoplasm (14). Normal cell cycles contain stages of controlled cell growth and proliferation as well as coordinated cell death referred to as apoptosis. The natural proliferation cycle of cells exists to create replacements for old or damaged cells and to compensate for nonpathological deficits which may occur. Cells that are old or damaged undergo a natural and automatic cell death thereby letting newer cells take over their biologic processes (14). The control for each of these processes is encoded in the DNA within the cell's genes. The balance of cell proliferation and apoptosis is altered as a result of multiple mutations in the DNA code over time (15). As a consequence of this prolonged imbalance a tumor develops. Classifying the cell as cancerous pertains to it having the characteristic of being malignant. A malignant tumor will infiltrate adjacent tissues as well as exhibit cell detachment from its initial location of origin, the neoplasm, move through blood vessels and the lymphatic system, and migrate to tissues and organs throughout the rest of the body (16). Tumors that do not exhibit these invasive characteristics are referred to as benign. This process of migration and infection is called metastasis. Metastasis is the greatest threat to the health and survival of the patient and is subsequently the most detrimental 
characteristic of cancer (17). Although metastasis of a melanoma can occur quickly, it is not immediate. There are eight defined stages of metastasis beginning with the primary tumor.

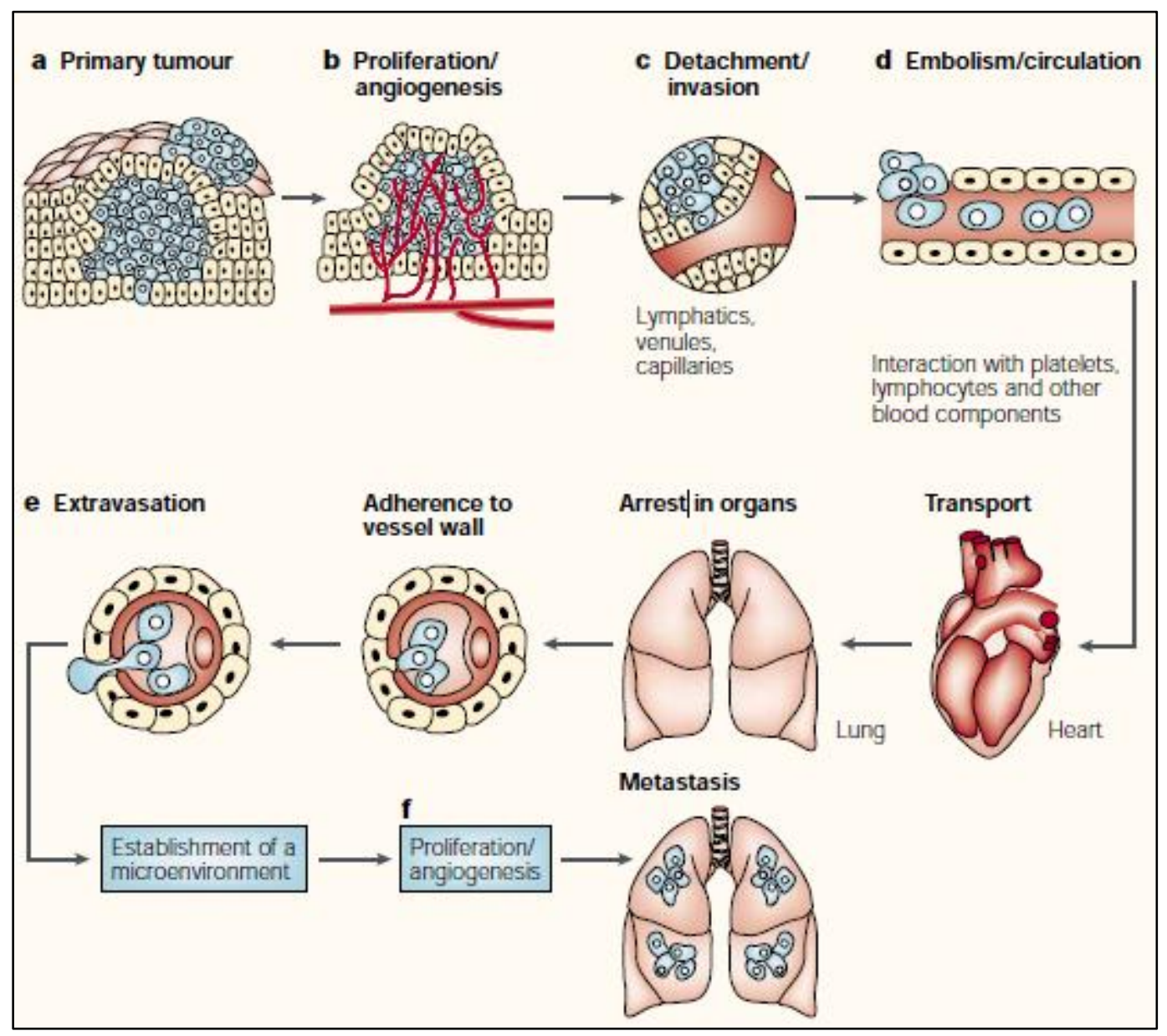

Figure 1.1 The eight stages of tumor growth and expansion leading up to metastasis (17).

The first stage is characterized by the primary growth of the neoplastic cells; at this stage the tumor expands. Proliferation and angiogenesis dominate the second stage. In order for the tumor to expand beyond a diameter of $2 \mathrm{~mm}$ vascularization must occur. For every increase in the amount of cellular proliferation a corresponding increase in new capillary formation must also occur (18). Following capillary development tumor cells will begin to detach from the primary mass and penetrate the surrounding healthy tissue eventually reaching a blood vessel (19). Once the blood vessel has been reached the cancer cells are transported throughout the body and proceed 
to attach to the capillary beds of other tissues and organs where the metastasizing tumor cell will breach the endothelial cell barrier and invade $(19,20)$.

There are a number of risk factors associated with developing cancer, they fall into two categories: environmental and genetic. Environmental factors encompass anything not strictly considered to be genetic. Some examples of environmental factors are chemicals, food, infection, radiation, obesity, cigarette smoking, stress, artificial tanning, and sunlight (21). Skin cancer is especially associated with UV damage as the result of long term sun exposure (4). Genetic factors include fair skin, previous melanoma, and a family history of cancer. Several genetic mutations in genes responsible for controlling cell growth can lead to tumor development. These mutations, such as BRCA1 and BRCA2 mutations related to breast and ovarian cancer, have been found to be hereditary (22).

\subsubsection{Diagnosis of Skin Cancer}

Current methods for assessing the type of tumor cell, benign or malignant, start with a biopsy. Skin biopsies are implemented in one of four ways. The choice of biopsy type is dependent upon the initial visual assessment of the lesion. Shave biopsies involve the use of a thin blade to shave off the uppermost layer of the area in question. Punch biopsies implement a sharp hollow circular tool to extract a thick section of the skin containing the first few top layers. An incisional biopsy employs a scalpel to remove a partial section of the growth and an excisional biopsy uses a scalpel to remove all of the growth including a portion of the surrounding tissue (4).

Each of the three types of skin cancer are a the result of mutations within a specific type or layer of the epidermis: squamous cell carcinoma develops from mutations in the keratinocytes of the stratum spinosum, basal cell carcinoma results from mutations of cells within the stratum basale, and cancerous melanoma develops from mutations within melanocyte cells. 


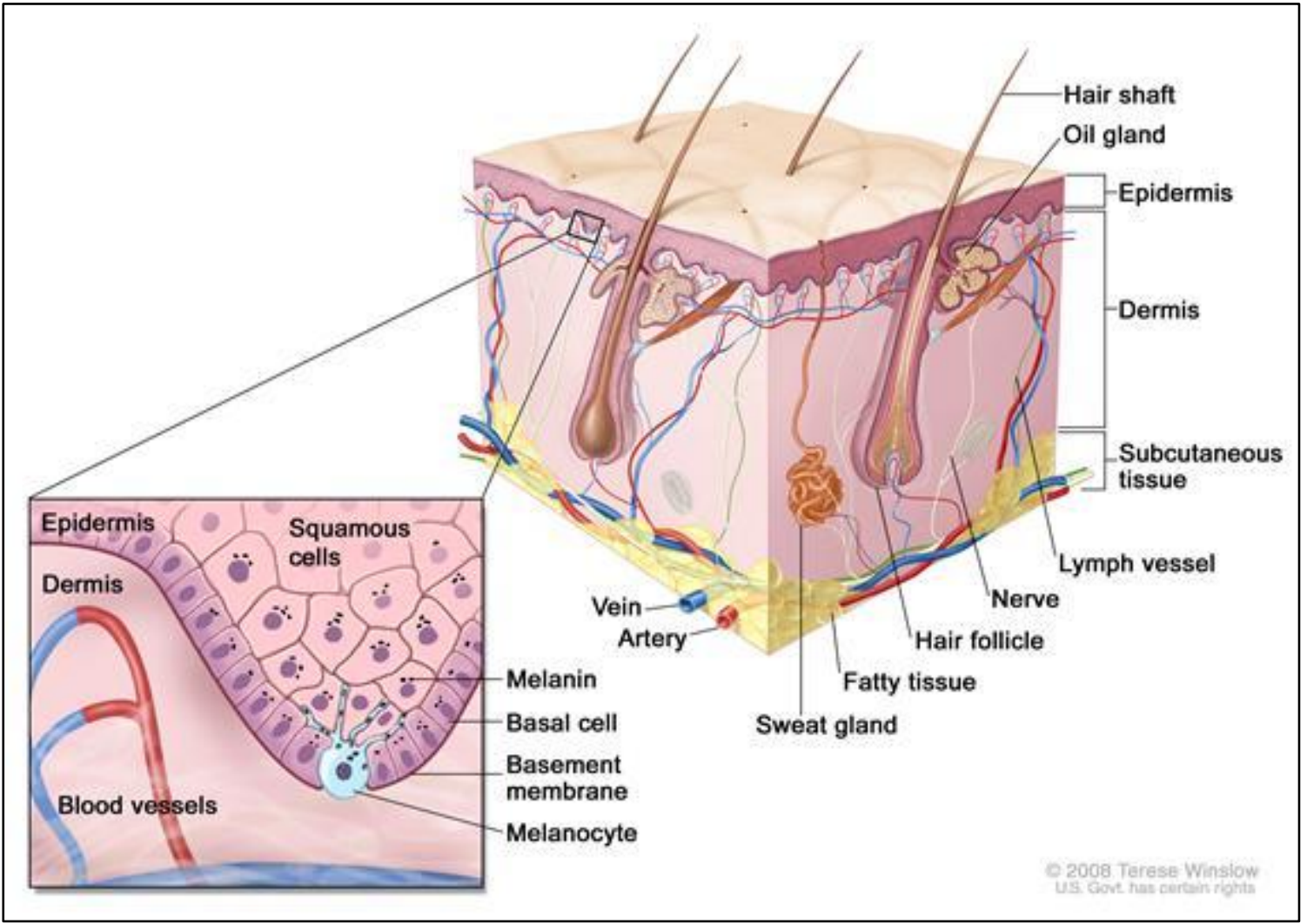

Figure 1.2 The epidermal and dermal layers of the skin with a magnified view of the epidermal layer in which the squamous cell, basal cell, and melanocyte reside (21).

Of these three, basal cell carcinoma (BSC) is the most common but also the least dangerous (23).

BSC is classified as a non-melanoma cancer as a result of its extremely low probability for

metastasis (24). BSC develops in the stratum basale, a single layer of stem cells and keratinocytes intermixed with melanocytes and tactile cells. The stratum basale layer is the deepest layer of the epidermis, resting on the basement membrane just above the dermis. In cases of BSC, the lesion begins as a "small shiny bump" and upon enlargement will acquire a depression at its center. If allowed to progress the lesion will eventually invade the dermis (24). Squamous cell carcinoma (SCC) presents as rough, scaly, flat, reddish patches and most often affects those of fair complexion and extended chemical or sun exposure over their lifetime (25). SCC develops in the keratinocytes of the stratum spinosum and without treatment may eventually metastasize to the lymph nodes (24). Melanoma is the deadliest skin cancer as a result of its high rate of metastasis 
and is therefore the leading cause of skin disease related death, but constitutes only $5 \%$ of the total cases of skin cancer $(26,24)$. Melanoma is caused by mutations in the melanocytes ordinarily responsible for producing the pigment melanin.

\subsubsection{UV Radiation}

The electromagnetic (EM) spectrum encompasses the entire range of known radiation energies starting with the most energetic forms, gamma, $\mathrm{x}$-ray, and $\mathrm{UV}$, and progressing to the least energetic forms such as infrared and radio waves (27). The energy radiated from a source is directly linked to the wavelength of the emitted radiation measured in units of nanometers (nm). The longer the wavelength of the emitted radiation, the lower the associated energy of the emitted radiation, conversely, a shorter wavelength of emitted radiation will result in a higher energy and therefore the more harmful the effects of the emitted radiation.

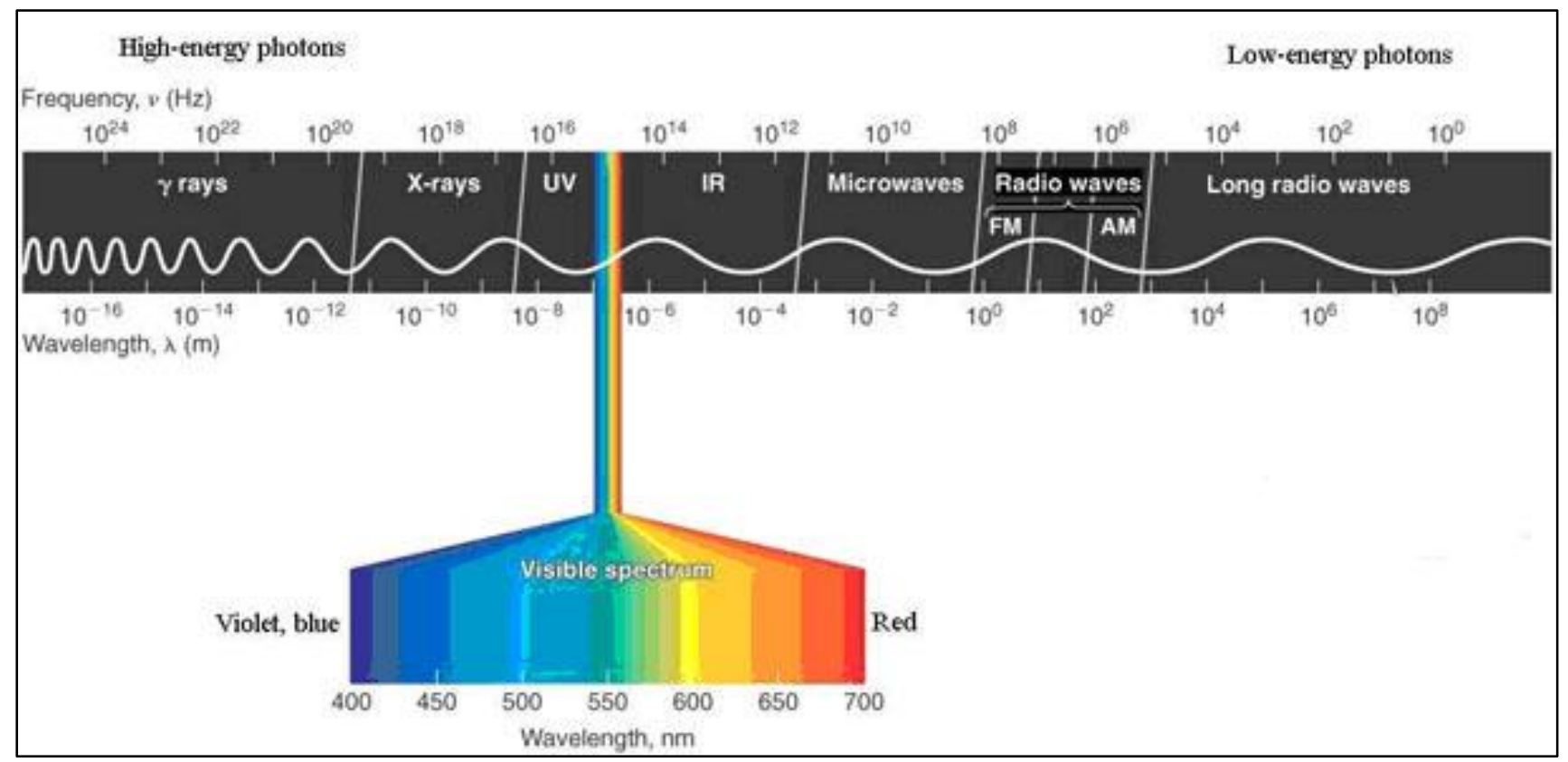

Figure 1.3 The electromagnetic spectrum (28).

The EM spectrum range, in order of lowest to highest energy, is: radio waves, microwaves, infrared, visible, ultraviolet (UV), x-rays, and gamma rays (27). The UV range of the spectrum can 
be further divided into near UV (UVA), far UV (UVB), and extreme UV (UVC) $(36,37)$. The most prevalent source of UV radiation is the sun. UVC radiation has a wavelength between $100-290 \mathrm{~nm}$ and is completely blocked from reaching the surface of the Earth as a result of the ozone layer of the atmosphere absorbing the radiation (37). UVB rays, 290-320nm, are partially absorbed by the ozone layer and are therefore partially transmitted to the surface. UVA, (320-400nm) rays pass through the ozone layer without any attenuation and are thereby fully transmitted to the surface of the Earth (37). Although UVB radiation has been determined to be the more carcinogenic of the two, extended exposure to both UVA and UVB radiation, results in damage to DNA thereby causing mutations which may lead to the formation of melanomas (38).

\subsection{The Epidermis}

The skin is the largest organ of the human body, making up roughly $15 \%$ of total body weight and covering a surface area of 1.5 to $2.0 \mathrm{~m}^{2}$ in adults (24). Skin is composed of two layers, the epidermis and the dermis; the epidermis being the outermost of the two. One of the main functions of the epidermis is to act as a barrier to the harmful effects of UV radiation (24). In this capacity the epidermis protects the deeper tissue layers form exposure to the damaging UVA and UVB rays. The epidermis consists of a series of cell layers, each containing keratinocyte cells in various states of growth and viability. Keratinocytes make up the majority of the cells found in the epidermis, approximately $90 \%$, and are named as a result of their primary function of synthesizing keratin $(24,39)$. 


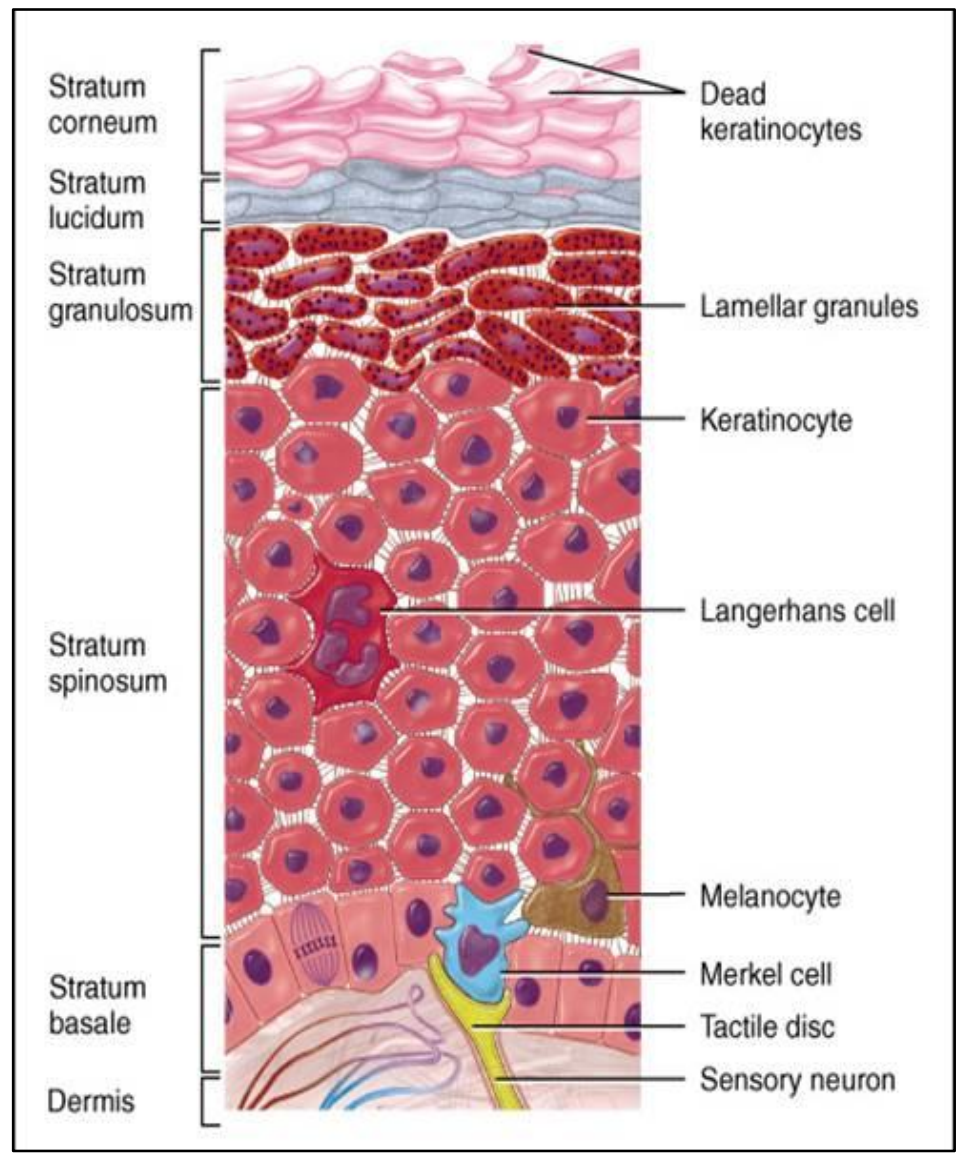

Figure 1.4 The five layers of the epidermis and the cells that comprise each layer (39).

Keratinocytes, originating in the stratum basale layer as a product of stem cell and keratinocyte cell mitosis, migrate upwards through each subsequent layer of the epidermis toward the surface where the replace damaged or dead epidermal cells in a 30 to 40 day cycle of regeneration (24).During the migration process the shape and function of the keratinocyte cells changes. As they move up through the layers they become flatter and produce a lipid-filled membrane-coating vesicle that aids in waterproofing the epidermal surface (24). Continued exposure of epidermal cells to UV radiation can lead to mutations, which over time can begin affecting the cells ability to control proliferation leading to the formation of cancerous lesions. 


\subsection{Phospholipids}

The plasma membranes of cells separate intracellular components from extracellular components as a means of maintaining the environment necessary for cellular function. The plasma membrane is composed of a phospholipid and phosphoglyceride bilayer integrated with a variety of associated proteins (40). A phospholipid is an amphiphilic molecule comprised of a polar hydrophilic head and two non-polar hydrophobic acyl fatty chains (40). The hydrophobic heads of the phospholipid are positioned outward creating both the intracellular and extracellular surfaces of the membrane. The hydrophilic acyl fatty chains are directed inward and embedded within the membrane layer.

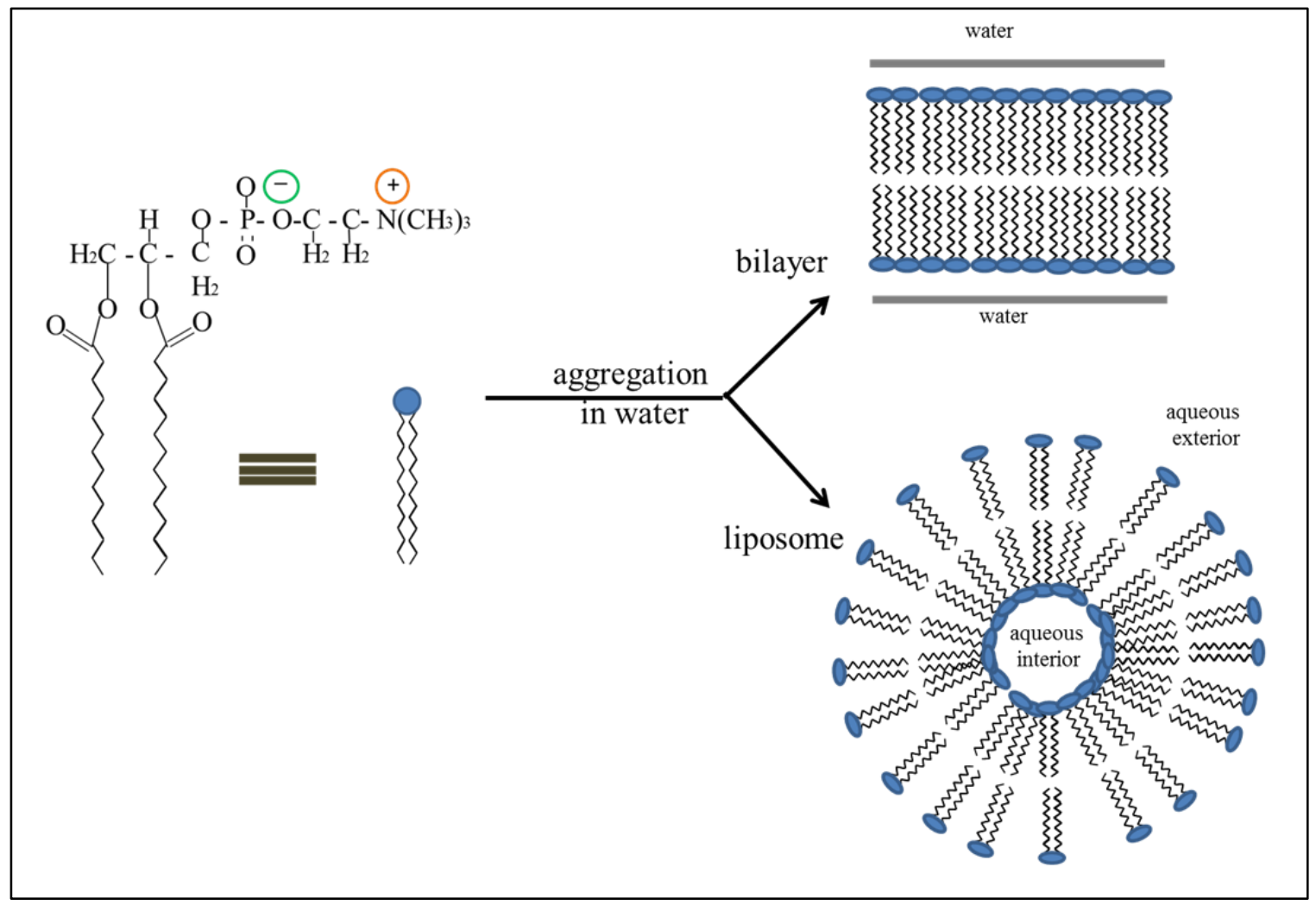

Figure 1.5 Phospholipid molecular structure and aggregation formation in the presence of water (41).

The species designation of phospholipid is dependent upon the particular alcohol either attached to or forming the primary backbone of the molecule. In the case of phosphatidylcholine 
(PC), phosphatidylserine (PS), phosphatidylinositol (PI) and phosphatidylethanolamine (PE), their alcohols are attached to the glycerol backbone of the molecule. Unlike PC, PS, PI, and PE, the alcohol of the phospholipid sphingomyelin (SP) is the backbone of the molecule (40). The phospholipid composition of the membranes allows for diffusion within the plane of the membrane (40). It is this quality, promoting the integration of other phospholipid based structures, that can be exploited in the effort to tag and map tissue using phospholipid encapsulated QDs constructs. Additionally phospholipids can be altered to contain specific functional headgroups such as poly ethylene glycol (PEG) and amines $\left(-\mathrm{NH}_{2}\right)$ to increase the biocompatibility of the phospholipids and provide additional sites for further bioconjugation (11). As a result of the amphiphilic nature of the phospholipids, one hydrophobic end and one hydrophilic end, they are especially useful as a material for encapsulating the normally toxic and hydrophobic inorganic CdSe/ZnS QDs. The amphiphilic characteristic inherent to phospholipids makes it possible for them to attach to the QDs on one end and interact with an aqueous environment on the other end. Typically when phospholipids are suspended in a polar nonorganic substance such as water they will spontaneously associate to form bilayers like those illustrated in Figure 1.6 (42). When suspended in an organic substance, such as chloroform, phospholipids become soluble at room temperature and their hydrophobic fatty acid chains can readily attach to the like-wise hydrophobic surface of the QD. Multiple attachments of phospholipids in this manner result in a QD that is completely encapsulated and therefore able to become soluble in aqueous solvents. 


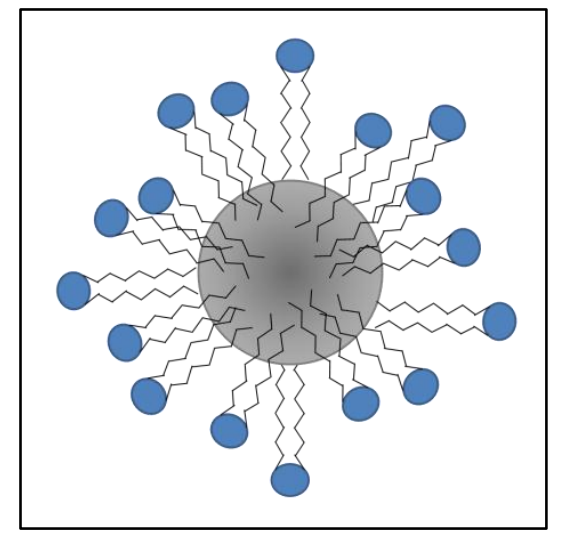

Figure 1.6 Phospholipid fatty acid chain attachments to QD surface.

\subsection{Quantum Dots}

All materials can be characterized by their tendency to either promote the movement of electrons through their mass, or to inhibit the movement of electrons through their mass; their degree of electrical conductivity (43). Those that promote the movement of electrons are referred to as conductors and those that inhibit the movement of electrons are referred to as insulators. Intermediary between insulators and conductors are materials classified as semi-conductors. The distinguishing characteristic between insulators, conductors, and semi-conductors is the size of their respective band gaps.

The band gap describes the energy needed to be absorbed by an electron in order for that electron to be excited to the conduction band, as well as the energy released by the electron when it de-excites back to its ground state in the valence band. The relative ease of promoting an electron from the valence band to the conduction band determines the electrical conductivity of the material (43). The Fermi energy denotes the highest energy level occupied by an electron when at absolute zero. Understanding the connection between atomic orbitals, electron energies, valence shells, and band gaps is integral to understanding the fundamental phenomenon responsible for the unique emission behavior exhibited by quantum dots. 


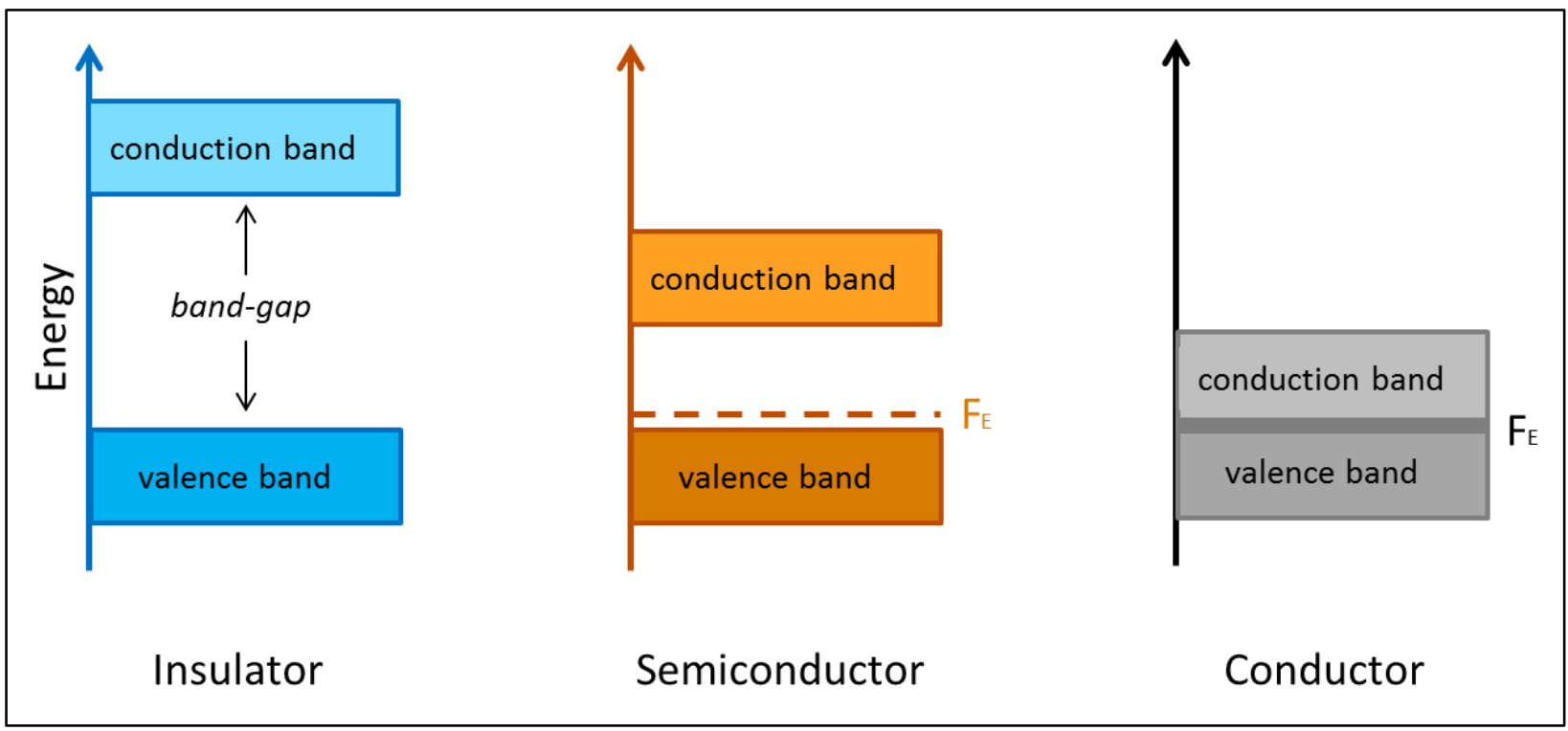

Figure 1.7 Variation of band gaps between the conduction and valence bands of insulators, semiconductors, and conductors.

Insulators have empty conduction bands and a valence band full of electrons tightly bound to their atoms by ionic or covalent bonds (44). Covalent bonding, the sharing of valence electrons between neighboring atoms, occurs in materials composed of a single element or similar elements; ionic bonding is the complete transfer of an electron from one atom, an electron donating metal, to another atom, an electron accepting non-metal (45). The presence of these strong bonds between the electron and the atom result in a large band gap, an inhibition to electrical conduction, necessitating the need for a significant amount of energy to induce an electron to transition to the higher energy conduction band. In the case of pure diamond 6 to $7 \mathrm{eV}$ is required to excite a single electron into the conduction band (44). Conductors possess little to no band gap between their valence and conduction bands and often the highest available, but unoccupied, energy levels of the valence band will overlap with the lowest empty energy levels of the conduction band. This overlap causes the electrons within the partially filled valence band to move freely within the material promoting conductivity (44). 
Semiconductors are a type of material characterized by their inhibition of electron movement at low temperatures and their promotion of electron movement as more energy is put into the material, often in the form of an increase in temperature from absolute zero, but also as the result of incident radiation (45). The central property that defines a material as conducting, semiconducting, or insulating is the band gap of the material. The band gap of the material is an intrinsic property of the bulk material. The change in this intrinsic material property relative to the number of constituent atoms, bulk state or nano-scale, is key to the fluorescent property of quantum dots.

The Aufbau principle describes the characteristic preference of electrons to occupy the lowest energy state possible (46). As such, electrons will fill the inner most atomic energy levels of an atom first. Each electron occupies a specific atomic orbital, within a specific energy shell, and the further out the energy shell is from the nucleus of the atom the greater the energy attributed to the electrons occupying that shell (47).The highest energy level occupied by an electron, when it is in its non-excited ground state, is the valence shell. Electrons occupying the valence shell are called valence electrons.

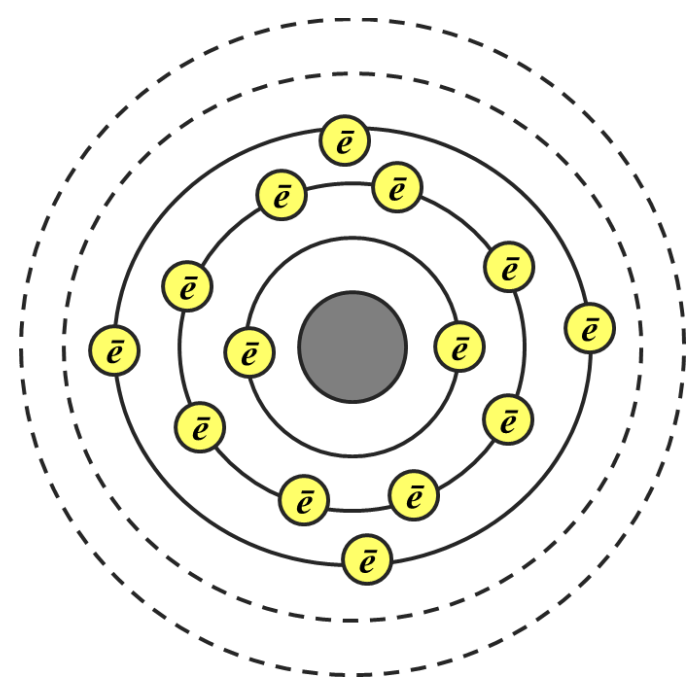

Figure 1.8 Electron atomic orbital lowest energy configuration for silicon (48). 
Valence electrons have the greatest probability of being promoted to a higher energy level (45). It is from the position of lowest energy, the valence shell, that an electron may be excited into a higher, and as yet unoccupied, energy. Excitation will occur if the electron is acted upon, and absorbs, an amount of energy equal to or greater than the difference in energy between the valence shell and the next higher energy level. The exact number of electrons permitted in each energy level and the minimum possible energy of an atom can be determined by coupling the aufbau principle with Pauli Exclusion Principle which states, "No two electrons in a quantum system can occupy the same quantum state" (49). Combining these two principles, unperturbed electrons will always occupy states of lowest energy and no more than two electrons can simultaneously occupy an atomic orbital, provides information on the exact energy of the highest energy valence electron. Knowing the exact energy of the highest energy valence electron, while also knowing the energy of the nearest unoccupied orbital, yields the effective band gap energy. In bulk form, the discrete energy states of the individual atom are replaced by the continuous valence and conduction energy bands (50). The Pauli Exclusion Principle prevents any two electrons form occupying the same quantum energy state, therefore, when multiple atoms are in close proximity, or bounded to one another, the discrete energy levels of the individual atoms form a band of multiple degenerate orbitals of the same energy for which the corresponding electrons can reside (47). Figure 1.9 shows an illustration of this progression from discrete energy levels to continuous energy bands. 


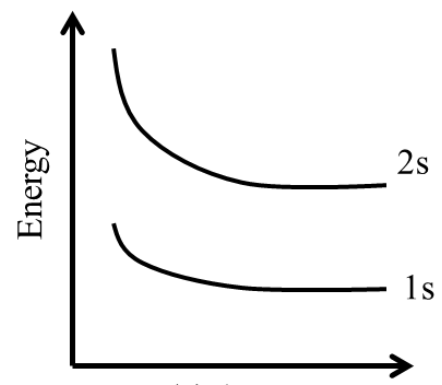

(a) 1 atom

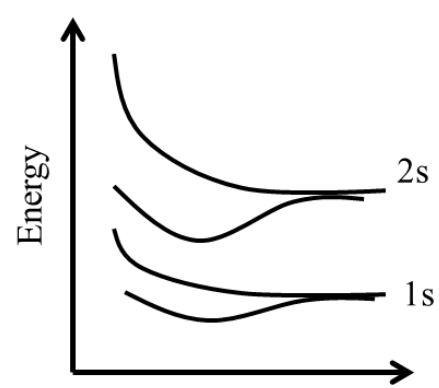

(b) 2 atoms

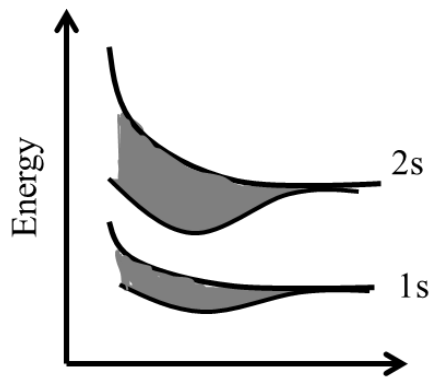

(c) $\mathrm{N}$ atoms

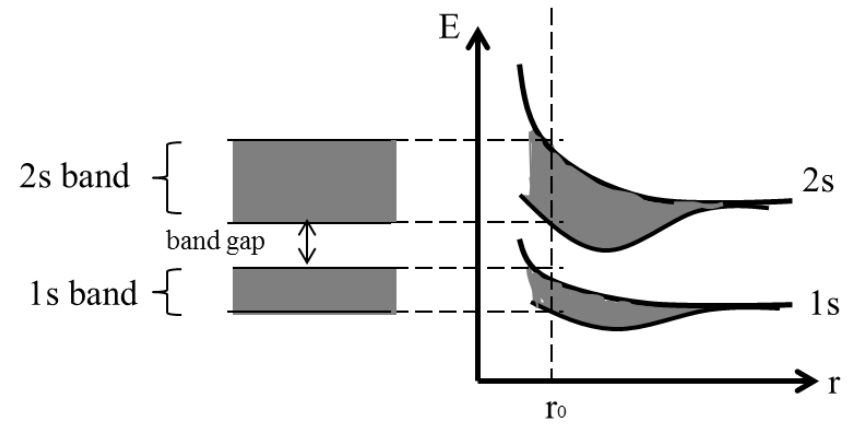

Figure 1.9 Progression from discrete energy levels to continuous energy bands during aggregation of individual atoms to form bulk material (47).

For a material to be considered a nano-material its largest dimension must be no bigger than $100 \mathrm{~nm}$, conversely, a material that is larger than $100 \mathrm{~nm}$ with respect to its smallest dimension will, for the purposes of this thesis, be considered a bulk material (45). Quantum dots are nano-crystals composed of semiconducting material, which as the result of their small nano-scale size, 1-10nm, exhibit properties that are not observed in the larger bulk material. QDs have the same elemental composition and atomic arrangement as the corresponding bulk material; however, their size causes the number and arrangement of atomic energy levels available to electrons to be altered.

The valence and conduction bands found in the bulk material are the result of the interaction of the electron energy levels in the constituent atoms from which the bulk material is comprised. In the case of a bulk material, where thousands or millions of atoms are bound to one another, energy levels of individual atoms are forced to interact with energy levels of their 
surrounding neighbors. Interaction between the electron energy levels of each atom and its nearest neighbors are what create the continuous valence and conduction bands found in bulk materials rather than the discrete energy levels in atoms (47). Understanding the parallels between the energy bands that exist in the bulk material and the energy shells that exist in individual atoms will facilitate a general understanding of the quantum mechanical effects that are a consequence of the diminished size of QDs.

\subsubsection{Quantum Confinement and the Bohr Exciton Radius}

QDs have the same elemental composition and atomic arrangement (1) on the nano scale as the corresponding bulk material, however, decreasing the physical size of a material has several interrelated consequences: the number of atoms within the material is decreased, less bulk material means less space for the electron-hole pair to move in thus causing confinement of the electron, and the number of energy levels available to the electrons decreases and discrete atomic energy levels form rather than continuous energy bands.

In semiconducting bulk materials, when a photon possessing an energy greater than or equal to the band gap energy is absorbed, an electron will be excited in to the conduction band. The promotion of the electron from the ground state into a higher energy state results in a positively charged hole. Until the electron is de-excited and recombines with the hole, the pair can move about the bulk material but will remain within a prescribed distance of one another as a result of the Coulomb attraction between them (40). The distance between the electron and the hole is a unique characteristic intrinsic to each material referred to as the Bohr exciton radius.

In the case of $\mathrm{CdSe}$ cores the average distance between the electron and corresponding hole is 5.6nm (51). If a CdSe crystal smaller than this distance is synthesized the energy is constrained and quantum confinement of the energy will develop. A continued decrease in the size of the QD relative to the natural electron-hole distance causes an increase in the amount of confinement and 
subsequently an increase in the band-gap energy. Increases in the band gap energy result in a transition from the continuous energy bands of the bulk material back toward the discrete energy levels seen in atoms. This atom-like resemblance of the discrete energy levels of QDs has promoted use of the term "artificial atom" when referring to QDs (51).

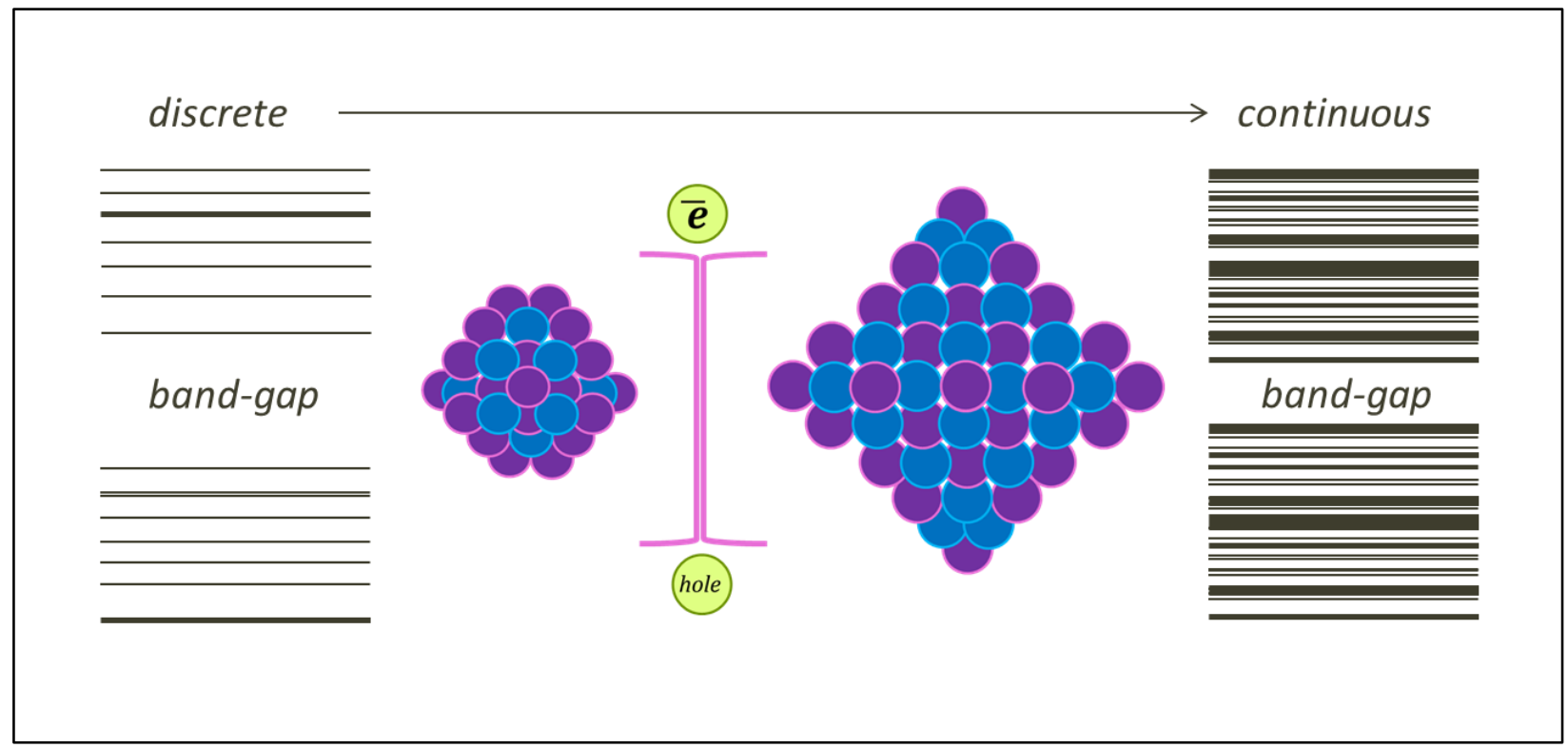

Figure 1.10 Relationship between size, Bohr exciton radius, band gap and composition of atomic energy levels.

The progression of electron movement restriction leading to quantum confinement can be accomplished in two ways: physical reduction in the size of the material or trapping the material between layers of a second material with a larger band gap energy. As the physical size of the material is decreased, composed of fewer and fewer atoms, the energy states of the atoms are no longer as compressed, they lose the continuous quality found in the band gaps of the bulk material, they start to spread out and become more discrete. A standard illustration for the change in energy states of a material as the size of the material decreases is given in Figure 1.11. In the bulk material, referred to as $3 \mathrm{D}$, the energy state is continuous, the electron can move in all three 
dimensions. By restraining the electron transitions in one dimension, either by the physical removal of the bulk material or by using a secondary material with a larger band gap, the electrons are now confined to two dimensions. This confinement alters the manner in which the electron energy states interact, creating distinctions between each energy state. As we continue on, the states become more well-defined until finally they exhibit the same discrete quality as individual atoms.

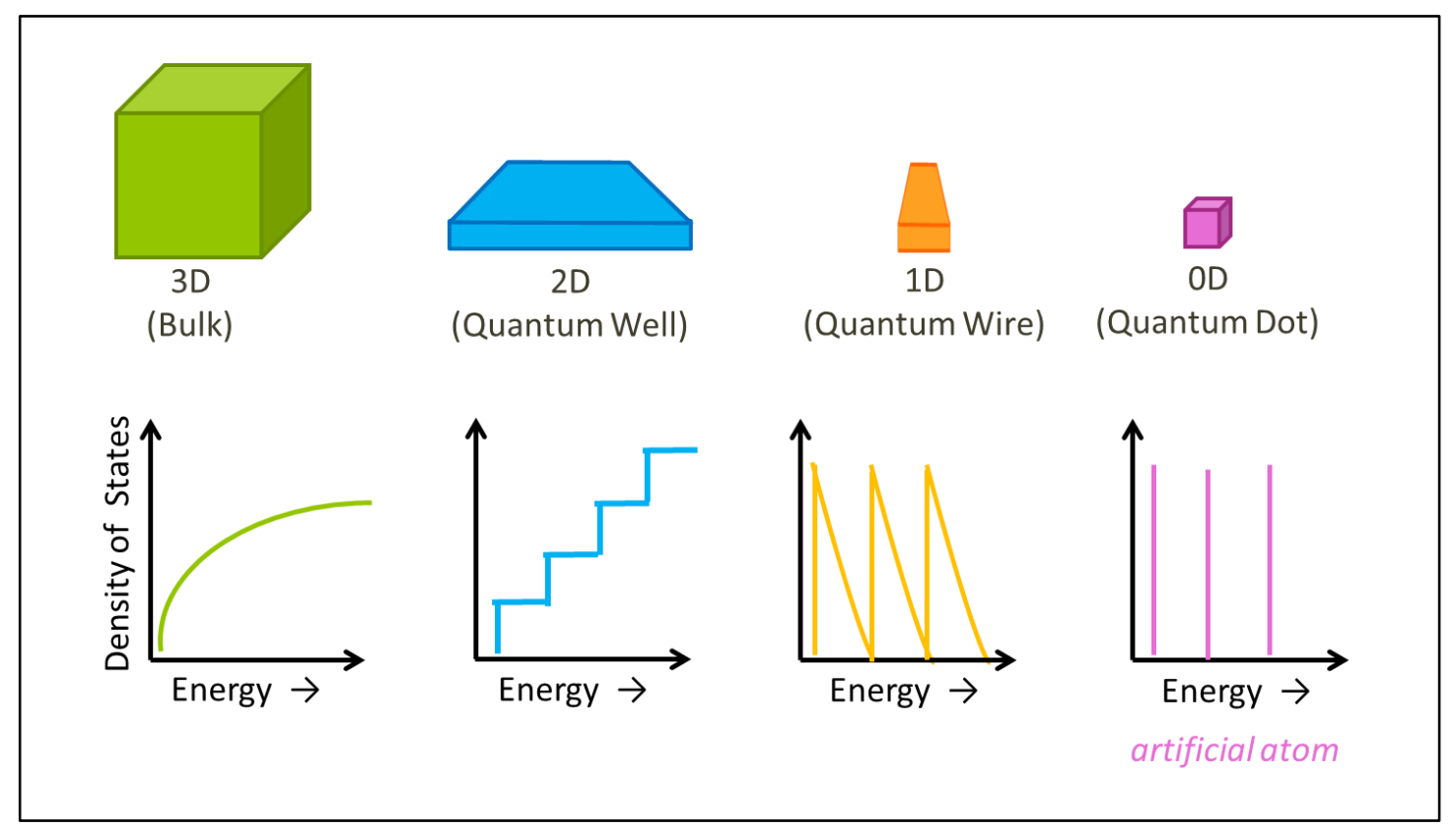

Figure 1.11 Progression of energy state densities as a result of increased electron energy confinement.

This transition toward discrete energies associated with a reduction in the size of the material is directly related to the aforementioned Bohr exciton radius. When the size of the material is less than the distance of the exciton radius, the electron's wave-function is constrained and the subsequent effect is an increase in the band gap (52). As the size decreases, the electron wave function becomes ever-more confined, as the electron wave function becomes ever-more confined the band gap energy increases, and as the band gap energy increases the energy needed to excite an electron from the valence to the conduction band also increases; each of which result in the 
increased energy of the emitted photon. Keeping these relationships in mind, the connection between QD size and photon emission wavelength can be made. Relative to the electromagnetic spectrum, the higher the energy of the emitted photon the more shifted toward the blue end of the spectrum and the lower energy more toward the red. Figure 1.12 illustrates this connection between QD size and emission wavelength. The basic break down being, small QDs have bigger band gap energies, bigger band gap energies result in higher energy emitted photons, therefore the energy of the emitted photon is directly related to the size of the QD; by synthesizing QDs of a specific size you can tune the wavelength of emitted light.

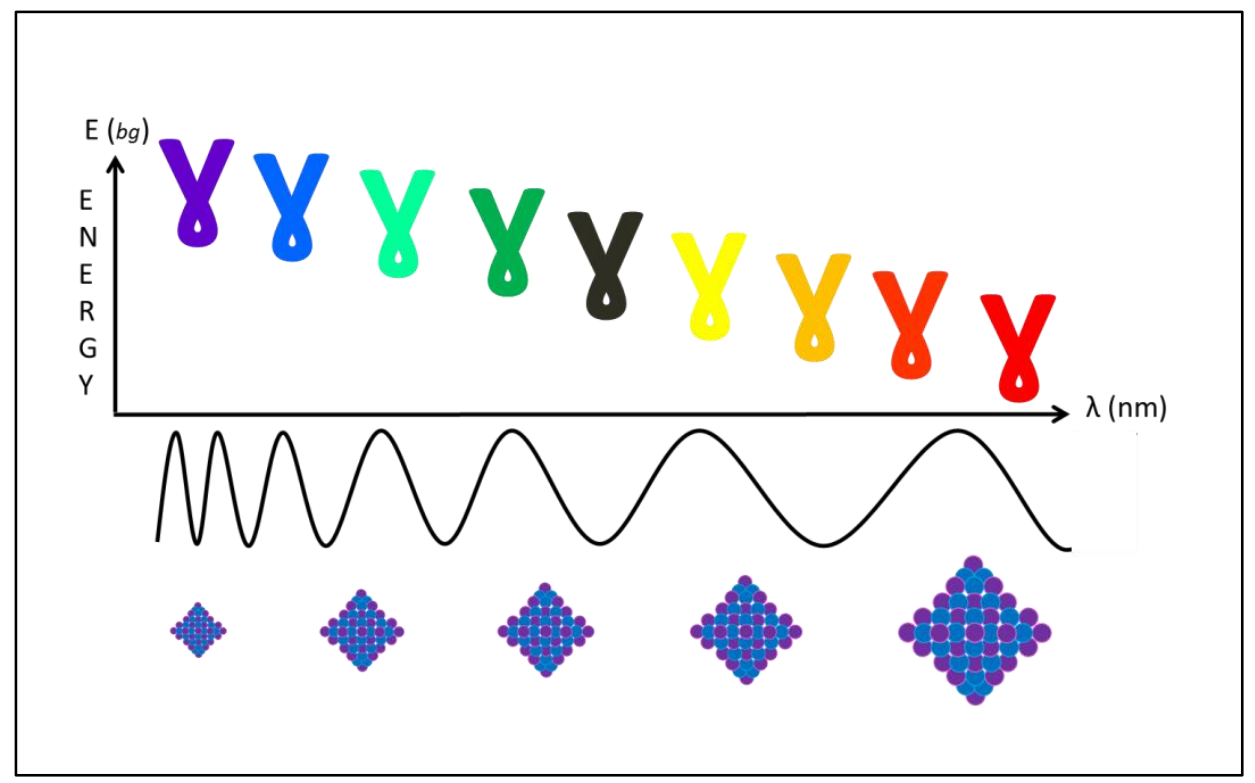

Figure 1.12 Relationship of QD size band gap energy and photon emission wavelength.

\subsubsection{Biologic Quantum Dot Applications}

Current Biomedical QD applications for QDs include membrane labeling, fluorescent microscopy, antibody tagging, genomic analysis, metabolic analysis, and diagnosis of metabolic processes $(10-13,53,54)$. QDs are promising substitutes for organic fluorophores in many of the 
biologic applications involving membrane labeling, fluorescent microscopy, and antibody tagging. One of the most prominent advantages of using QDs over their organic counterparts is the QDs reduced tendency to photo-bleach and the possibility for exciting multiple QDs of different emission wavelengths with the same excitation source. This capability to use a single excitation source for multiple QDs facilitates imaging of samples in which there are several components of interest. QDs are being used in genomic applications as well. Samples containing different combinations of QDs with distinct emission spectra conjugated to different DNA probes are mixed in a solution with a target DNA containing complementary sequences (55). The hope is that this process can be fine-tuned for detecting the presence of genetic defects. Similarly, viral analysis can be undertaken using what is referred to as QD bar codes - ten different QD colors could conceptually provide 45 different QD pair combinations $(56,57)$. The discrete wavelengths of QD emission make this possible, specifically tailoring them through functionalization to target multiple sites upon a tumor increasing the probability of detecting even the smallest amounts of cancerous cells. QD-fluorescent dye conjugates are also proposed as tools in the diagnosis of metabolic processes. They provide a way to monitor the proteolytic activity of extracellular matrix metalloproteinases $(58,59)$. These examples are a small sample of the variety of biologic applications in which the use of QDS has started to become common place; a range of applications that is increasing every day.

\subsection{Overview of Thesis Goals and Deliverables}

This introductory chapter has served as a vehicle for providing the foundational knowledge and background pertinent to this thesis. The clinical problem of detecting and diagnosing cancer at the earliest stages of development is, in cases of the most aggressive forms of cancer, paramount to patient survival. False negatives which may occur during the examination of biopsied lesions are 
counterproductive to the goal of early detection. Current methods relying on visual examination of biopsied samples viewed under a microscope are inadequate for detecting small populations of the abnormal cells indicative of cancer. A more adequate method of detection is required; functionalized QD constructs are a mechanism which can be employed to fill this need. QD constructs have the potential to not only identify and target cancerous cells, but eventually to also be used simultaneously as drug delivery devices.

The purpose of this thesis was to develop a foundational method for the solubilization and primary functionalization of QDs to be used in biologic tagging. This was accomplished through encapsulation of QDs of different emission wavelengths within phospholipid micelles. To achieve this, CdSe QDs were synthesized using the standard Cal Poly QD synthesis protocol with minor modifications in synthesis temperature and reaction time to achieve QDs of the desired emission wavelengths. These CdSe QDs were then coated in $\mathrm{ZnS}$ shells at a variety of synthesis temperatures to once again produce QDs with an emission wavelength specific to the necessary testing parameters. Fluorescence spectra were used to assess the center max wavelengths (CMWs), intensities, and full width at half max associated with each batch of synthesized QDs. Choice of QDs to be encapsulated and used in the keratinocyte cell culture trials was dictated by the emission wavelengths of the fluorescent stains applied to the keratinocyte cultures: Calcein at 517nm and Ethidium homodimer-1 at $635 \mathrm{~nm}$. The QDs were then encapsulated in phospholipid micelles and introduced to the keratinocyte cultures of varying periods of time. This thesis offers a first method for the solubilization and primary phospholipid functionalization of QDs for introduction to keratinocyte cells that can be further modified for future applications. 


\section{Chapter 2 - Development and Evaluation of CdSe/ZnS Quantum Dots}

\subsection{Introduction}

In 1970 Japanese physicists Esaki and Tsu were the first to produce crystalline superlattices possessing near perfect match of lattice constants leading to the creation of a two dimensional structure with previously unseen electronic properties $(60,61)$. Also in the early 1970s researchers at AT\&T Bell Laboratories and IBM began making 2D quantum wells using semiconducting materials (62). In 1984 Miller et. al. at AT\&T Bell Laboratories in New Jersey published research on the shifts in optical absorbance experimentally found in GaAs-AlGaAs quantum well structures contrasting the effects with bulk semiconductors as a "consequence of quantum confinement carriers” (63). Louis E. Brus of AT\&T Bell Laboratories discovered quantum dots in colloidal solutions, at the same time Efros and Ekimov at the A.F. Ioffe Physical-Technical Institute in St. Petersburg Russia were also developing semiconducting nanocrystal technology, first discovered in a glass matrix, which was later christened "quantum dots" by Mark A. Reed in 1988 (64, 65). In the early 1990s the field nanocrystal research was expanding and synthesis using both top down and bottom up techniques were being employed by multitudes of researchers.

The top down approach involves working with a macroscopic volume of material and gradually and precisely decreasing its size, either through mechanical or chemical means, until the nanoscale, approximately $100 \mathrm{~nm}$, has been reached. The top down process is akin to that of a whittler slowly shaving down a hunk of wood into a delicately carved miniature rocking chair (66). The top down approach to synthesizing QDs, though effective, is time consuming and expensive (67).

Bottom up approaches include self-assembly, colloidal synthesis, and guided attachment of colloidally synthesized QDs through plasma lithography (67). Self-assembly of QDs can be 
achieved by electron beam lithography, a physical mechanism by which an electron beam etches grooves into a substrate that are then filled by the semiconducting material, molecular beam epitaxy (MBE) where the semiconducting materials are heated to the point of vaporization and condensed and reacted on a substrate, or metal-organo chemical vapor deposition (MOCVD), a chemical mechanism in which the atomic deposition of one semiconducting material is applied on to a substrate of a second semiconducting material (68). Through natural processes involving the energetic stability associated with lower energy states the deposited material forms nanometer sized beads on the surface of the substrate. The final QD nanocrystals are produced by layering the beads with a semiconducting material possessing a band gap larger than that of the bead material (69). An added benefit to self-assembled or colloidally synthesized QDs using a bottom up approach, aside from faster synthesis time and decreased cost of materials, is that the resulting QDs have smaller diameters, and subsequently stronger quantum confinement potentials, than QDs synthesized using one of the top down approaches (69).

By the late 1990s the synthesis had shifted away from using the initial toxic and air-reactive chemicals toward "green synthesis" utilizing still biologically toxic but less hazardous and more stable reagents. Interest in the field of nanocrystals and quantum dots and their varied applications has grown exponentially over the last three decades as is evident from the number of related research published in journals; almost none pre-1980 to over 3500 per year by 2009 (64). Although research continues in both the top down and bottom up synthesis of quantum dots, the bottom up approach has overtaken in popularity.

As mentioned in Chapter 1, a variety of different starting materials are available and have been utilized for the synthesis of QD nanocrystals. Both the top down and the bottom up methods create QD nanocrystals that are composed of atoms from groups II - IV or III - V elements that are found in the periodic table, such as CdSe, CdTe, CdS, and ZnSe for groups II - IV, and InP and 
InAs for groups III - V (70). Of the many available semiconducting materials, the CdSe/ZnS core/shell combination has been in use in the colloidal synthesis of QDs the longest, since 1996 (71). The most well-known and commonly used protocols for the colloidal synthesis of quantum dots use the "green synthesis" variety of $\mathrm{Cd} / \mathrm{Se}$ compounds as the semiconducting starting material (72). The number of readily accessible articles outlining the numerous variations on the original protocol developed by Murray et al (73) is evidence of the interest and developing usefulness of QD involved applications.

A standard CdSe core synthesis procedure from the 2005 paper "A Safer, Easier, Faster Synthesis for CdSe Quantum Dot Nanocrystal" written by Nordell et. al. had been previously developed for use at Cal Poly by Aarron Lichtner in 2009 (74). The procedure was later expanded upon to include a $\mathrm{ZnS}$ coating step for increased quantum yield and stability. The currently used $\mathrm{ZnS}$ coating protocol was initially developed from literature by Cal Poly graduate student Sabrina Bruce-Akman in 2010 and later refined by Josh Angell in 2011. The effects of altering the nonreagent related parameters for synthesizing CdSe cores and applying the standard ZnS coating procedure were further investigated and modified for the purposes of this thesis. The previously developed Cal Poly standard synthesis procedure, in its entirety, can be found in appendix B.1 or Josh Angell's 2011 thesis Synthesis and Characterization of CdSe - ZnS Core - Shell Quantum Dots for Increased Quantum Yield. The previously developed bottom up colloidally synthesized CdSe/ZnS QD Cal Poly procedure was modified to achieve the QDs with the necessary emission wavelengths for tagging and mapping a biologic using phospholipid encapsulated QD constructs. For the purpose of tagging and mapping a biologic it was essential to have at least two sizes of QDs; to have QDs with two distinct and distinguishable emission wavelengths. For this reason knowledge of, and familiarity with, the QD synthesis process was necessary for maintaining a continuous supply of QDs for use in the phospholipid encapsulation procedure. Likewise it was 
important that the synthesis of the QDs be consistent and well documented. From the initial procedure, optimized protocols for the synthesis of white CdSe cores and shelled CdSe/ZnS QDs with emission wavelengths of $505 \mathrm{~nm}(+/-10 \mathrm{~nm})$ and $555 \mathrm{~nm}(+/-) 10 \mathrm{~nm}$ were developed. The resulting synthesized CdSe/ZnS QDs were used in the encapsulation procedure described in chapter 3.

\subsection{Methods}

\subsubsection{Synthesis of CdSe cores}

Synthesis of the CdSe cores applied the same reagents and amounts of reagents as described in the standard Cal Poly protocol. Each of the precursors, selenium trioctylphosphine (Se-TOP) and cadmium (Cd), were synthesized following the steps in the protocol, with only slight modifications pertaining to the method for measuring out appropriate quantities of each of the dry reagents. The standard procedure weighed out $33 \mathrm{mg}$ of selenium powder or $13 \mathrm{mg}$ of the cadmium oxide powder, into a plastic weigh-boat and then transferred the powder into a $50 \mathrm{~mL}$ round bottom flask. The method used in this thesis was to directly transfer the powder into the two neck 50mL round bottom reaction flask. The standard synthesis of the Se-TOP precursor yields a $5.4 \mathrm{~mL}$ volume of the precursor. The Se-TOP precursor for this work was synthesized in quintuple batches to reduce the variation across the multiple CdSe core synthesis procedures. Se-TOP precursor older than five days was discarded for use in core synthesis beyond the initial experimental trials. The size of the Cd precursor batch was dependent on the size and number of samples to be extracted. For one or two samples, of $5 \mathrm{~mL}$ each, a single batch was made; for extractions of three or more samples a double batch was made. During the initial testing of synthesis parameters, a quintuple batch of Cd precursor was made and divided up into $10 \mathrm{~mL}$ volumes for synthesis of the CdSe cores using a stock solution. This was done in an effort to eliminate additional variables during testing of 
experimental parameters. For both the Se-TOP and Cd precursor synthesis, all reagents were purged with nitrogen gas and vented simultaneously for a period of ten minutes. After combination of all reagents into the reaction flask the Nitrogen and vent needles were removed to prevent evaporation of the solution. For synthesis of the CdSe cores the $\mathrm{Cd}$ precursor was heated to the desired reaction temperature and upon reaching the reaction temperature the room temperature SeTOP precursor was added. The ratio of $\mathrm{Cd}$ to Se-TOP precursor used in each synthesis was the $10 \mathrm{~mL}$ of $\mathrm{Cd}$ to $1 \mathrm{~mL}$ of Se-TOP prescribed in the standard protocol. At the desired reaction times of $0,30,60$, or 90 seconds CdSe samples were extracted, using a non-disposable glass syringe, and deposited into the appropriate sample vials. After each CdSe sample had cooled qualitative examination of the sample was performed by exposure of the sample to a generic black light. Quantitative data was then obtained using an Ocean Optics USB4000 Spectrometer to take fluorescence readings of each sample.

\subsubsection{Experimental Variables Investigated for the General Synthesis of CdSe Cores}

Four variables were manipulated in the synthesis of the CdSe cores: temperature, reaction time, receptacle volume, and quenching. The temperature of the $\mathrm{Cd}$ precursor at the time of SeTOP injection was altered from the standard $225^{\circ} \mathrm{C}$ typically used, to 220,215 , and $210^{\circ} \mathrm{C}$. Two consequences resulted from the change in temperature; the obvious and primary effect was a decrease in the temperature at the time of Se-TOP injection. A secondary effect was a reduction in the time that the $\mathrm{Cd}$ precursor was exposed to heat before injection. It took less time for the $\mathrm{Cd}$ precursor to heat up to 220,215 , or $210^{\circ} \mathrm{C}$, approximately 4 minutes less for each five degree interval, providing a reduction in the overall time of exposure to heat. The reaction time of the CdSe core synthesis was defined as the time from complete injection of the Se-TOP into the heated $\mathrm{Cd}$ until the beginning of extraction into the glass syringe. Samples were extracted at times of 0 , 30,60 , and 90 seconds. The receptacle volume ranged from $2 \mathrm{~mL}$ to $15 \mathrm{~mL}$ with vial diameters of 1 , 
$1.5,2$, and $2.5 \mathrm{~cm}$. Variation of sample vial size was investigated to determine if heat dissipation could be improved. A variety of vial arrangements and configurations were designed that would provide alternate sample distributions for cooling. Along with altering the volumes of samples as a method to assist in heat dissipation, quenching methods were investigated. Comparisons of the differences in center max wavelength (CMW), peak intensity, and full width at half max (FWHM) of the fluorescence spectrums for samples cooled in air, the standard, and acetone were made for three time points: 30, 60, and 90 seconds. Quenching with acetone was carried out by flushing the outside of the vials with acetone. For a period of one minute the sample vials were lowered into and raised out of an acetone bath in 5 second intervals and then submerged for 3 minutes. On occasion if the vials were submerged longer than 3 minutes the CdSe solution inside would develop a slushy consistency.

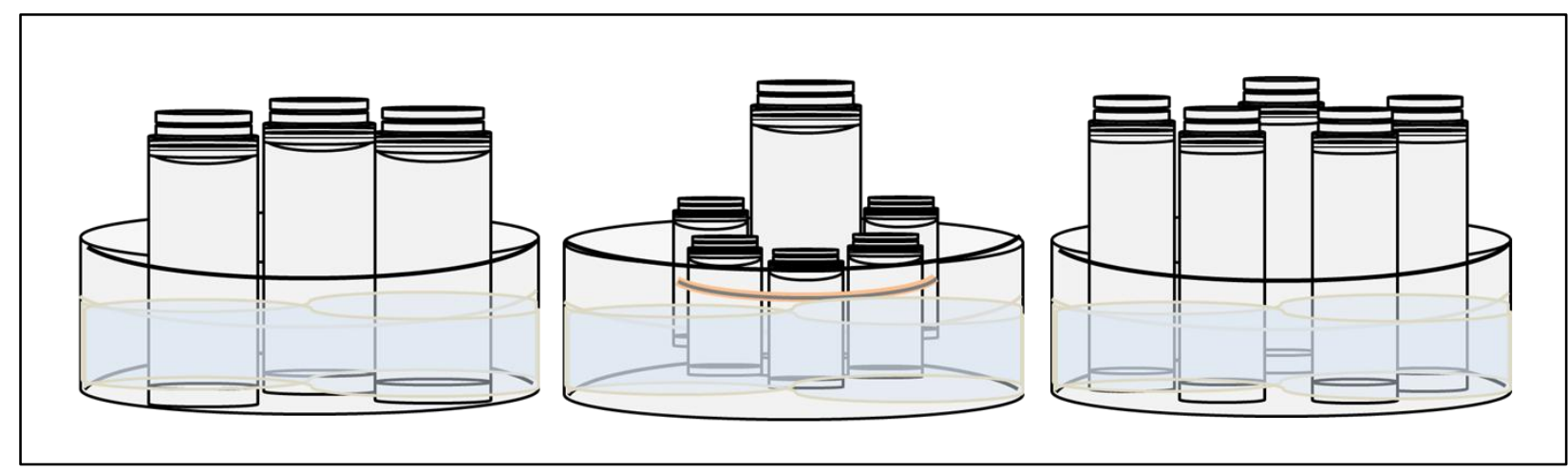

Figure 2.1 Three sample vial arrangement used to test the effects of volume size on CdSe fluorescence spectra. From left to right: $15 \mathrm{~mL} 2.5 \mathrm{~cm}, 2 \mathrm{~mL} 1 \mathrm{~cm}$, and $10 \mathrm{~mL} 1.5 \mathrm{~cm}$ diameter glass sample vials in an acetone bath.

\subsubsection{Synthesis of White Fluorescing CdSe Cores (WFCs)}

The same four variables were manipulated in the synthesis of the white fluorescing CdSe cores (WFCs). The two parameters particularly focused on were reaction time and quenching. 
The entire process of extracting the sample from the reaction flask to injecting it into the glass sample vial averaged 10 seconds. Reaction times varied between 0 and 15 seconds.

A 5cc glass syringe was used for extraction of the WFC samples. Practice and patience was required to remove the sample as quickly as possible. The glass extraction syringe was positioned in the second neck of the reaction flask and upon full injection of the Se-TOP precursor into the heated Cd precursor a 3-5mL sample was extracted and injected into 1-5 sample vials. After one minute of flushing with acetone, the vials were left submerged in an acetone bath for 3 minutes.

\subsubsection{Synthesis of ZnS Coated Quantum Dots}

The process used for the coating of the CdSe cores with $\mathrm{ZnS}$ shells applied the same reagents and amounts of reagents as described in the standard Cal Poly protocol. The ZnS precursor was synthesized following the steps in the standard protocol. The standard protocol did not specify a specific $\mathrm{CdSe}$ core solution to $\mathrm{ZnS}$ precursor ratio to be used. For the purposes of this thesis a standard ratio of $5 \mathrm{~mL} \mathrm{CdSe}$ to $1 \mathrm{~mL}$ of $\mathrm{ZnS}$ was applied. In the modified procedure $5 \mathrm{~mL}$ of CdSe core solution was added to a $50 \mathrm{~mL}$ round bottom reaction flask; a magnetic spin vane and temperature probe were also placed in the reaction flask. The CdSe core solution was mixed at the synthesis heat, either 160 or $110^{\circ} \mathrm{C}$, in a silicon ( $\mathrm{Si}$ ) oil bath for ten minutes. $1 \mathrm{~mL}$ of the $\mathrm{ZnS}$ precursor was added in a slow consistent drop-wise fashion over a period of 2 minutes. The reaction was then let to proceed for an additional 8 minutes. At the conclusion of the reaction time the solution was removed from the Si oil bath and cooled to $60^{\circ} \mathrm{C}$ at which point $1 \mathrm{~mL}$ of butanol was quickly added. The flask was then agitated side to side for 2 minutes. After each CdSe/ZnS sample had cooled, qualitative examination of the sample was performed by exposure of the sample to a generic black light. Quantitative data was then obtained using an Ocean Optics USB4000 spectrometer to take fluorescence and absorbance readings of each sample. 


\subsubsection{Temperature Variation of CdSe/ZnS Synthesis}

The effect of temperature on CMW during the synthesis of CdSe/ZnS QDs was investigated by comparing QDs synthesized at $70^{\circ} \mathrm{C}$, and $110^{\circ} \mathrm{C}$ in addition to the standard protocol synthesis temperature of $160^{\circ} \mathrm{C}$. In the comparison of the $\mathrm{CMW}$ of QDs synthesized at $70^{\circ} \mathrm{C}$ relative to those synthesized at $160^{\circ} \mathrm{C}, 4 \mathrm{WFC}$ samples were coated at $70^{\circ} \mathrm{C}$ and $4 \mathrm{WFC}$ samples were coated at $160^{\circ} \mathrm{C}$. The final CMWs, intensities, and FWHMs of the samples coated at $70^{\circ} \mathrm{C}$ were compared to those coated at $160^{\circ} \mathrm{C}$ by employing a two sample t-test. Figure 2.2 gives a schematic division of the ten previously synthesized CdSe core samples used for the comparison of the effects of QDs shelled at $110^{\circ} \mathrm{C}$ and at $160^{\circ} \mathrm{C}$. The ten samples consist of $5 \mathrm{CdSe}$ samples fluorescing white and five CdSe samples fluorescing green at 541nm with an intensity of 5800 counts and a FWHM of $44 \mathrm{~nm}$, that were combined within each set, creating two subsets each. The resulting sets provided two WFCs shelled at $110^{\circ} \mathrm{C}$ and at $160^{\circ} \mathrm{C}$, and two $541 \mathrm{~nm}$ samplesshelled at $110^{\circ} \mathrm{C}$ and at $160^{\circ} \mathrm{C}$ using the aforementioned shelling process. The CMWs of the resulting eight samples were then compared to the original corresponding CdSe core solution and to each other. 


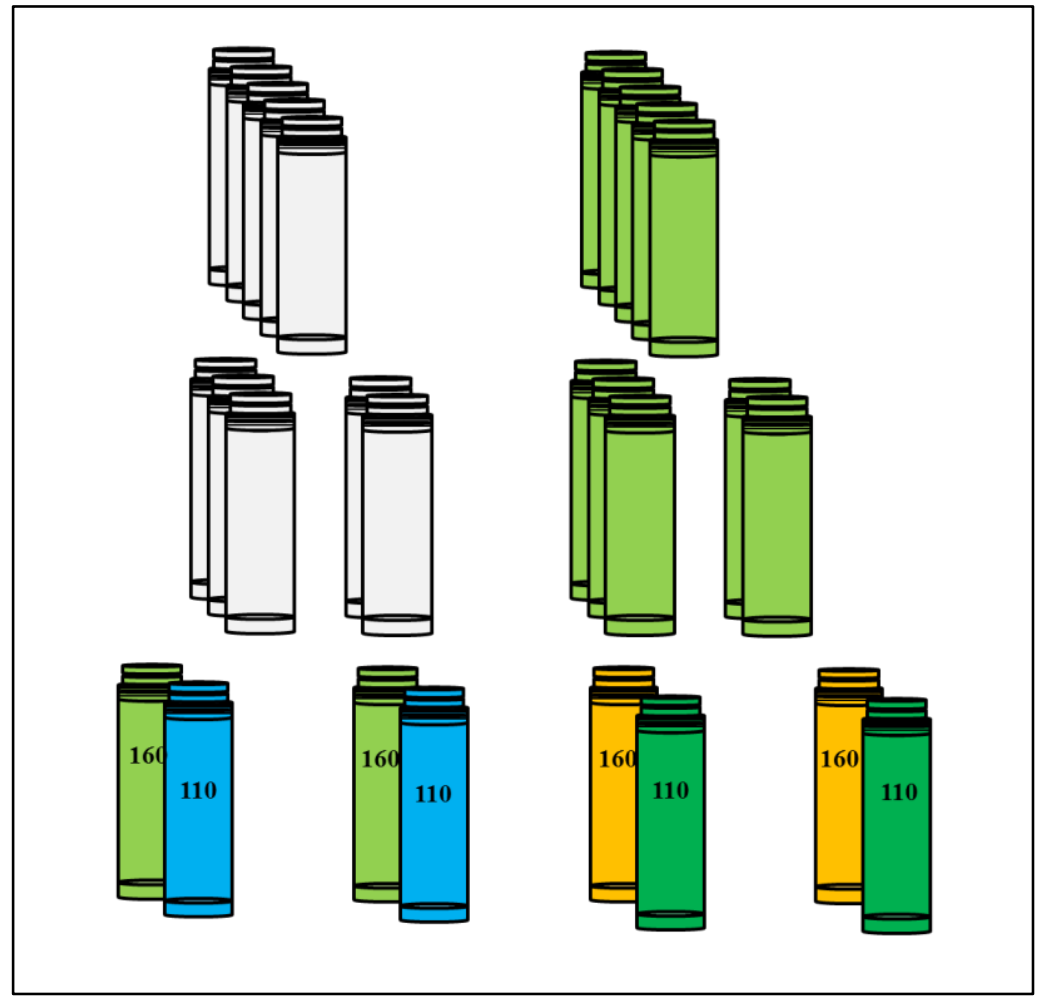

Figure 2.2 Schematic of the division of the ten previously synthesized CdSe core samples used for the comparison of the effects of QDs shelled at $110^{\circ} \mathrm{C}$ and at $160^{\circ} \mathrm{C}$.

\subsection{Results}

\subsubsection{Synthesis of CdSe Cores}

\subsubsection{Experimental Variables Investigated for the General Synthesis of CdSe Cores}

The receptacle volume only affected the outcome of the WFCs when the smallest and greatest volumes were compared and as a result of other possible contributing factors, could not be quantitatively demonstrated. The data in Table 2.1 are the CMW, Intensity, and FWHM averages of 23 different CdSe core samples used to compare the effects of the reaction temperature. Each average was taken using a minimum of three samples and a maximum of five samples. The visual representation of the data is given in Figure 2.3. Using the values from all of the samples for each time point, the standard deviation was calculated, using a p-value of 0.05 , and applied as a comparison of the averages for each time point in the form of error bars. Using standard deviation 
as a measure of the degree to which each parameter was affected by the synthesis temperature, it was initially concluded that for CdSe cores synthesized at 30,60, and 90s time points, and cooled in air, there was no statistically significant difference in the CMW, intensity, or FWHM.

Table 2.1 Effects of Temperature on CMW, Intensity, and FWHM for 30, 60, and 90s Samples Cooled in Air.

\begin{tabular}{|c|c|c|c|c|c|c|c|c|c|}
\hline & $\begin{array}{c}\text { CMW 30s } \\
(\mathrm{nm})\end{array}$ & $\begin{array}{c}\text { CMW 60s } \\
(\mathrm{nm})\end{array}$ & $\begin{array}{c}\text { CMW 90s } \\
(\mathrm{nm})\end{array}$ & $\begin{array}{c}\text { Intensity 30s } \\
(\text { counts })\end{array}$ & $\begin{array}{l}\text { Intensity 60s } \\
(\text { counts })\end{array}$ & $\begin{array}{c}\text { Intensity 90s } \\
(\text { counts })\end{array}$ & $\begin{array}{c}\text { FWHM 30s } \\
(\mathrm{nm})\end{array}$ & $\begin{array}{c}\text { FWHM 60s } \\
(\mathrm{nm})\end{array}$ & $\begin{array}{c}\text { FWHM 90s } \\
(\mathrm{nm})\end{array}$ \\
\hline $215^{\circ} \mathrm{C}$ & 523.5 & 539.4 & 550.5 & 7750 & 4700 & 2875 & 41.3 & 41.4 & 43.5 \\
\hline $225^{\circ} \mathrm{C}$ & 518.3 & 539.3 & 550.7 & 6867 & 4367 & 2833 & 37.1 & 39 & 39.7 \\
\hline $\mathrm{stdev}$ & 8.2 & 7.4 & 7.6 & 2360.0 & 1942.0 & 1452.0 & 3.4 & 5.8 & 8.2 \\
\hline
\end{tabular}

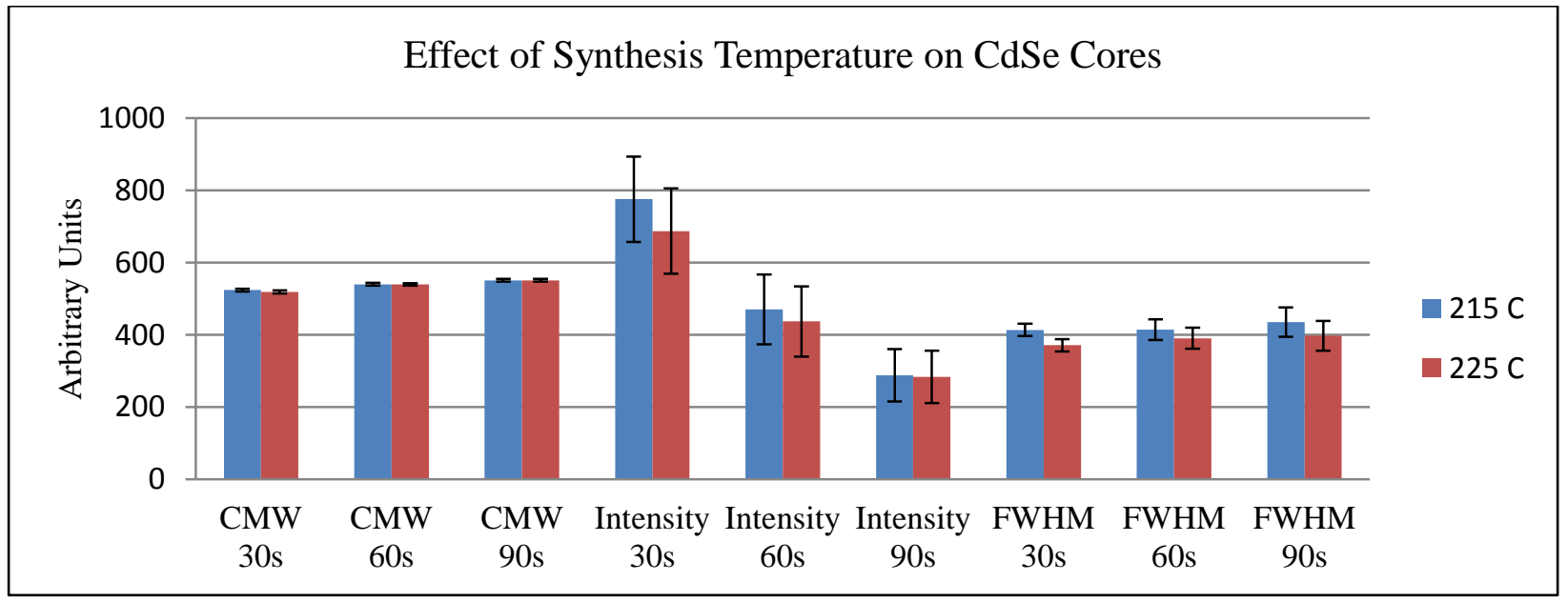

Figure 2.3 Comparison of the effects of reaction temperature on the average CMWs, intensities, and FWHMs of synthesis time points of 30,60, and 90s samples cooled in air.

In addition to calculating the standard deviation from all of the samples for each time point, a two sample t-test, assuming asymmetric variance, was conducted to compare the effects of temperature on the CMW, intensity, and FWHM for each time point of CdSe samples cooled in air. Table 2.2 gives the two-tail p-values associated with each t-test using an alpha of 0.05 and a confidence interval of $95 \%$. All of the t-test p-values were above the alpha value of 0.05 and therefore the null hypothesis, which indicates the means for each sample group were equal, could 
not be rejected. Based on this, it was again concluded that the temperature difference of $10^{\circ} \mathrm{C}$ at the time of injection of the Se-TOP precursor did not have a statistically significant effect on the CMW, intensity, or FWHM for time points of 30, 60, and 90s.

Table 2.2 Two Sample t-test of Effects of Temperature on CMW, Intensity, and FWHM for Each Reaction Time Point.

\begin{tabular}{|c|c|c|c|c|c|c|}
\hline $\begin{array}{c}\text { Quench } \\
\text { Medium }\end{array}$ & $\begin{array}{c}\text { Time } \\
(\mathrm{s})\end{array}$ & $\begin{array}{c}\text { Samples } \\
\text { at } 215^{\circ} \mathrm{C}\end{array}$ & $\begin{array}{c}\text { Samples } \\
\text { at } 225^{\circ} \mathrm{C}\end{array}$ & $\begin{array}{c}\text { CMW } \\
\text { p-value }\end{array}$ & $\begin{array}{c}\text { Intensity } \\
\text { p-value }\end{array}$ & $\begin{array}{c}\text { FWHM } \\
\text { p-value }\end{array}$ \\
\hline Air & 30 & 5 & 3 & 0.41 & 0.98 & 0.06 \\
\hline Air & 60 & 5 & 3 & 0.99 & 0.86 & 0.33 \\
\hline Air & 90 & 4 & 3 & 0.98 & 0.98 & 0.45 \\
\hline
\end{tabular}

\subsubsection{Temporal Effects on CMW, Intensity, and FWHM of CdSe Cores}

A series of synthesis, conducted at reaction temperatures of 215 and $225^{\circ} \mathrm{C}$, were performed to determine the effect that the duration of the reaction had on the CMW, intensity, and FWHM of the CdSe cores. Data for the CMW, Intensity, and FWHM averages of 23 different CdSe core samples that were used to compare the effects of the reaction times are given in Table 2.3. Each average was taken using a minimum of three samples and a maximum of five samples. The visual representation of the data is given in Figures 2.4 and 2.5. As a result of applying t-tests, it was previously determined that temperature did not play a statistically significant role in determining CMW, intensity or FWHM for 30, 60, and 90s reaction time points; for this reason all of the data points from both the 215 and the $225^{\circ} \mathrm{C}$ synthesis were used. The averages for the CMW, intensity, and FWHM of each time point were compared and a value for the standard deviation was calculated. The standard deviation was then applied in the form of error bars. The resulting data appeared to support the conclusion that there was a significant statistical difference in the CMW, 
and intensity of the CdSe cores which was dependent on the reaction time, but not a significant difference in the FWHM associated with each reaction time. To further support this conclusion a single factor ANOVA test was conducted.

Table 2.3 Averages of, and Standard Deviations for, the CMW, Intensity, and FWHM of CdSe samples for 30, 60, and 90s Reaction Times. Left: averages. Right: standard deviations.

\begin{tabular}{|c|c|c|c|}
\hline $\begin{array}{c}\text { Reaction } \\
\text { Time }(\mathrm{s})\end{array}$ & CMW (nm) & $\begin{array}{c}\text { Intensity } \\
\text { (counts) }\end{array}$ & $\begin{array}{c}\text { FWHM } \\
(\mathrm{nm})\end{array}$ \\
\hline 30 & 521 & 6838 & 41 \\
\hline 60 & 539 & 4575 & 41 \\
\hline 90 & 551 & 2857 & 42 \\
\hline
\end{tabular}

\begin{tabular}{|c|c|c|c|}
\hline $\begin{array}{c}\text { Reaction } \\
\text { Time }(\mathrm{s})\end{array}$ & $\begin{array}{c}\text { CMW } \\
(\mathrm{nm})\end{array}$ & $\begin{array}{c}\text { Intensity } \\
\text { (counts) }\end{array}$ & $\begin{array}{c}\text { FWHM } \\
(\mathrm{nm})\end{array}$ \\
\hline 30 & 8.2 & 2366 & 5 \\
\hline 60 & 7.4 & 1942 & 3.4 \\
\hline 90 & 7.6 & 1452 & 5.9 \\
\hline
\end{tabular}

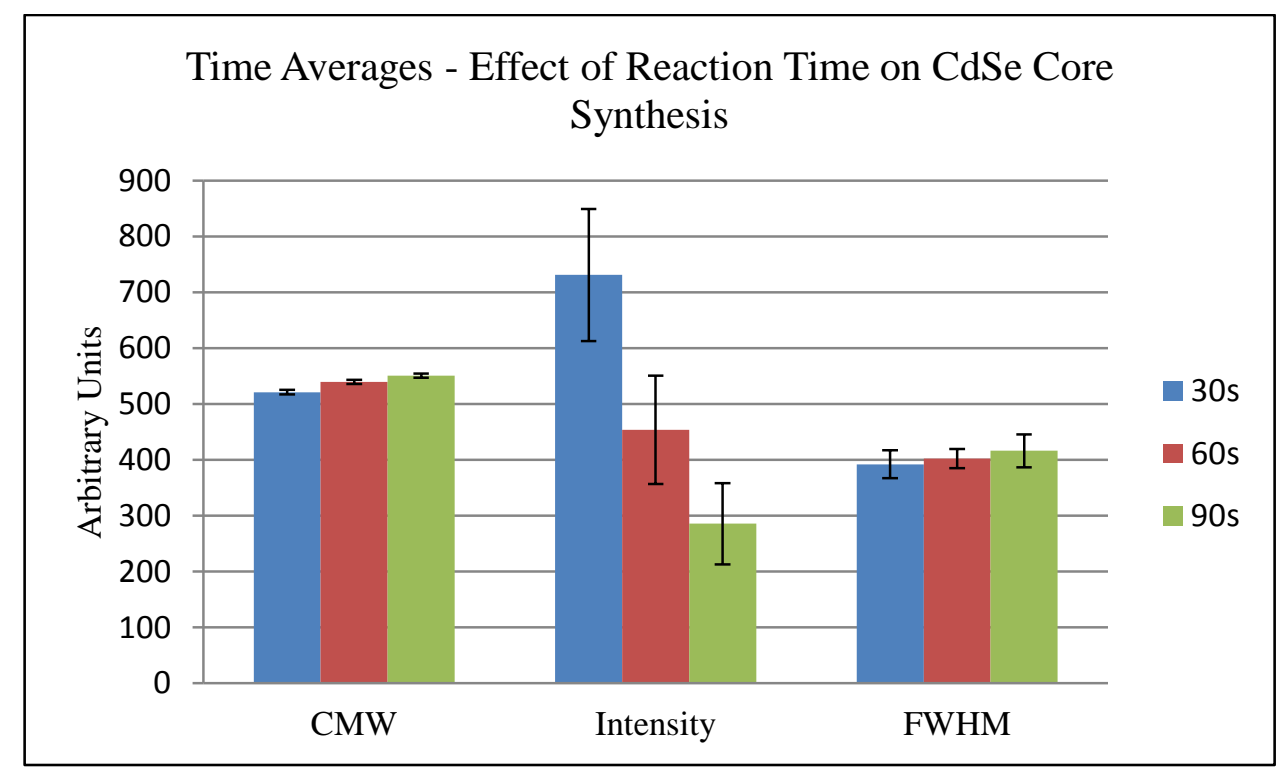

Figure 2.4 Combined reaction time data for 215 and $225^{\circ} \mathrm{C}$, quenched in air, averaged for each reaction time point. Standard deviations were calculated for each series of samples corresponding to individual reaction time points. 


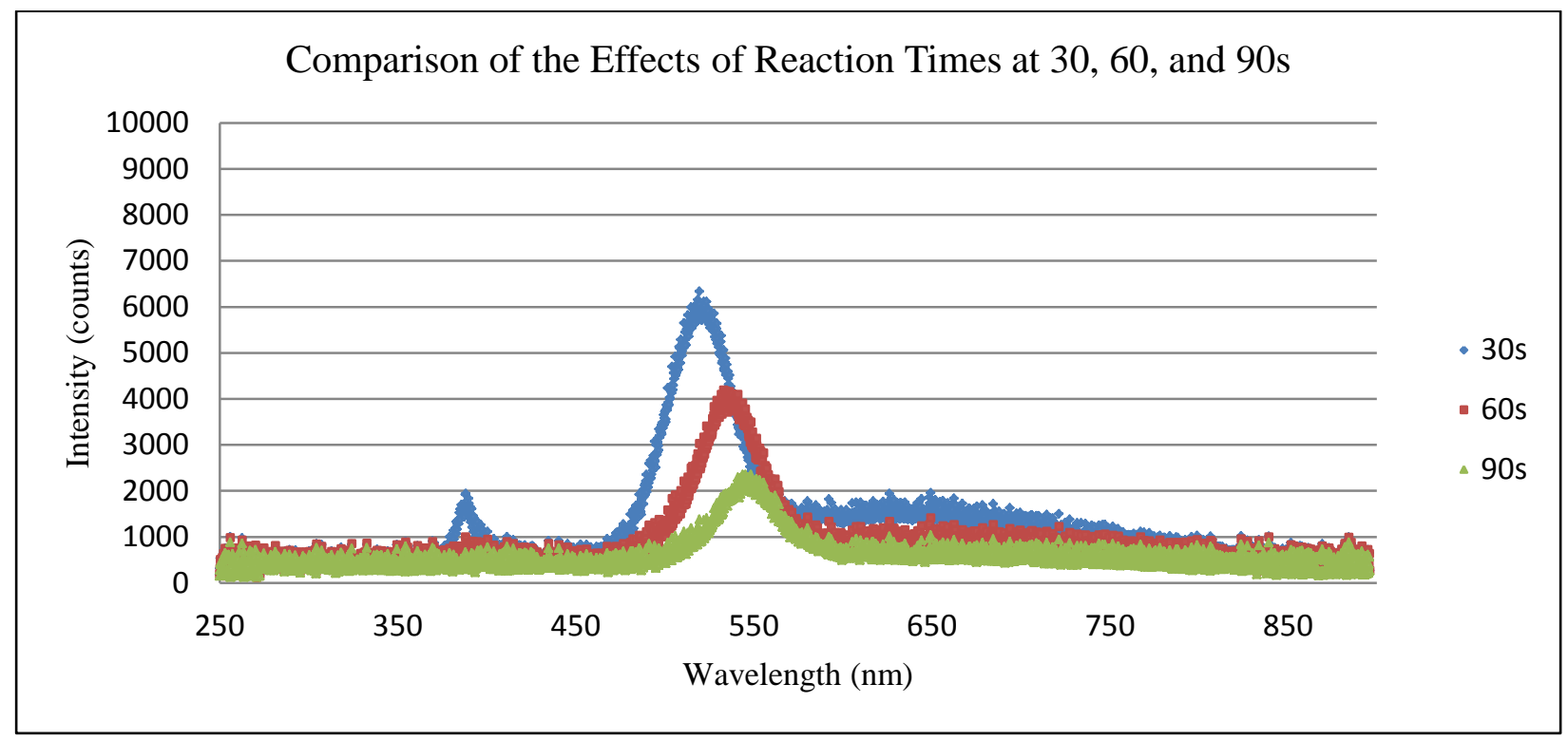

Figure 2.5 Fluorescence spectrum of CdSe core solutions for 30, 60, amd 90s reaction times.

A single factor ANOVA test was employed to determine the effect of different time points on the CMW, Intensity, and FWHM. Using the resultant p-values from the ANOVA tests it was concluded that the null hypothesis, that the means were equal within a 0.05 alpha value, could not be rejected for the FWHM; there was no statistically significant difference between the FWHM for each of the different reaction times. However, there was a statistically significant difference for the intensity and CMW of the reaction times with p-values of 0.003 and $2 \mathrm{E}-6$ respectively. These values support the conclusion that the reaction time of the CdSe core synthesis plays an integral role in determining the CMW and intensity of the CdSe core solution. 
Table 2.4 Single Factor ANOVA Test p-values for CMW, Intensity, and FWHM of CdSe Cores at Reaction Times of 30, 60, and 90s.

\begin{tabular}{|c|c|c|c|c|c|}
\hline $\begin{array}{c}\text { Samples } \\
\text { at 30s }\end{array}$ & $\begin{array}{c}\text { Samples } \\
\text { at 60s }\end{array}$ & $\begin{array}{c}\text { Samples } \\
\text { at 90s }\end{array}$ & $\begin{array}{c}\text { CMW } \\
\text { p-value }\end{array}$ & $\begin{array}{c}\text { Intensity } \\
\text { p-value }\end{array}$ & $\begin{array}{c}\text { FWHM } \\
\text { p-value }\end{array}$ \\
\hline 8 & 8 & 7 & $2.00 \mathrm{E}-06$ & 0.003 & 0.86 \\
\hline
\end{tabular}

A series of CdSe core synthesis were conducted to illustrate the dramatic change in CMW that different reaction times could elicit. Ten different $\mathrm{CdSe}$ core samples were synthesized at 0 and 60s, five samples for each reaction time, using the same starting Cd and Se-TOP stock solutions. Figures 2.6 is a digital image of the series as it appears under a generic black light. Figure 2.7 gives a corresponding fluorescence spectrum comparing the averaged spectrums of the series.

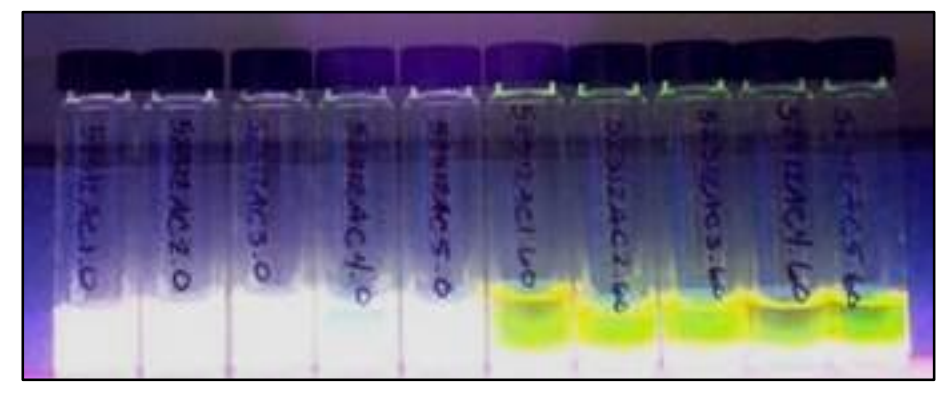

Figure 2.6 Digital image of CdSe core samples synthesized at 0 (white fluorescing) and 60 seconds (green fluorescing) viewed under black light. 


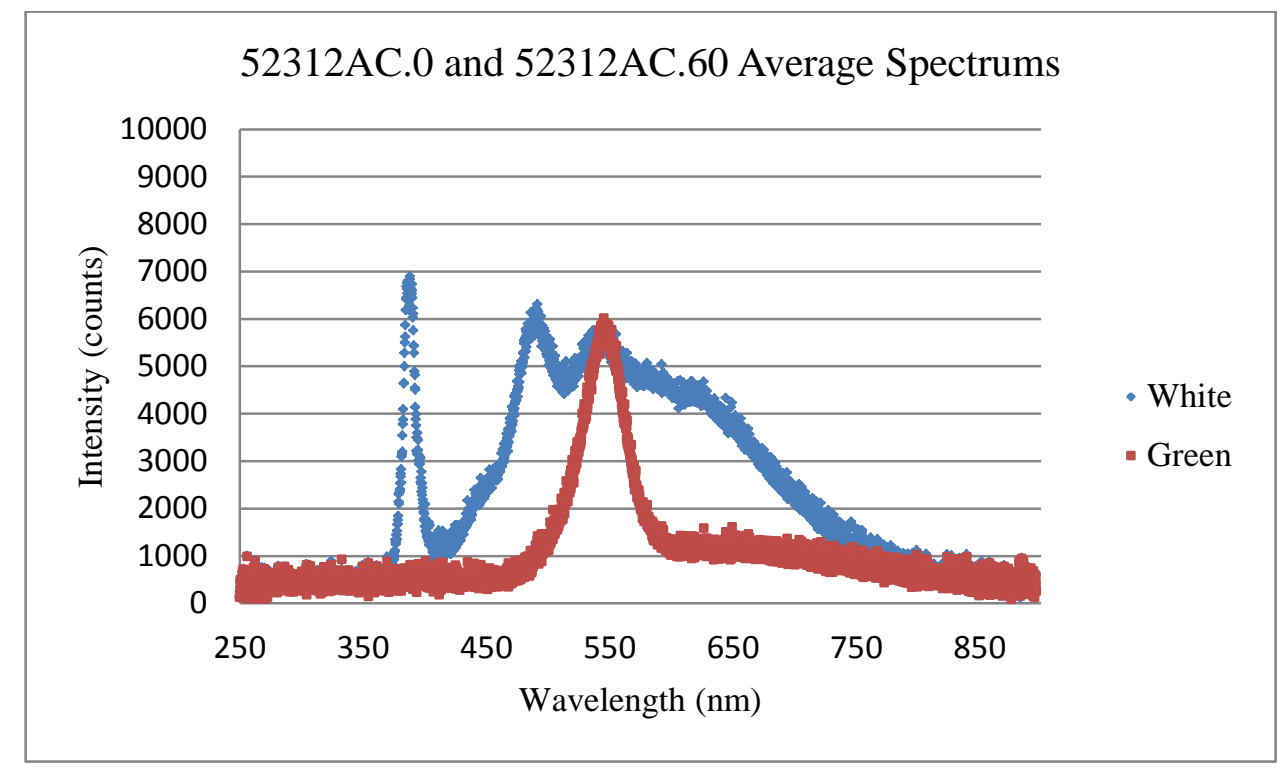

Figure 2.7 Fluorescence spectrums of averaged CdSe core sample sets synthesized at 0 (white fluorescing) and 60 seconds (green fluorescing).

A series of comparisons were conducted using $24 \mathrm{CdSe}$ samples to compare the effects of quenching samples in acetone versus the standard procedure that employed no additional quenching measures; the standard procedure was classified as quenching in air. A two sample t-test was used for the comparison of acetone versus air in three different synthesis arrangements: 30 s reaction time point at an injection temperature of $215^{\circ} \mathrm{C}, 60$ s reaction time point at an injection temperature of $215^{\circ} \mathrm{C}$, and a 60 s reaction time point at an injection temperature of $225^{\circ} \mathrm{C}$. The resulting p-values, corresponding to an alpha of 0.05 , were used to determine the significance of the data and are given in Table 2.5. The only variable that showed a statistically significant difference, p-value less than 0.05 , was the intensity for the sample set with the 30 s reaction time synthesized at $215^{\circ} \mathrm{C}$. There are three explanations for this: the sample sized used was too small to yield an accurate representation of the effects and/or the effects are only significant at smaller reaction times, or the results are accurate. Each sample set contained a minimum of three samples, as three samples are traditionally the very minimum number required to conduct a legitimate t-test; it is possible that a larger sample set would alter the corresponding p-values. If the effect of 
quenching with acetone is only significant at shorter reaction times, including data from samples synthesized at a reaction time of 15 seconds could aid in supporting such a conclusion. If the data is accurate as is, then the conclusion based on the p-values is that quenching with acetone only has a significant effect on the intensity of CdSe cores synthesized at reaction time points of 30s, noting that a comparison of all the intensity p-values may indicate a the existence of a possible trend for increased effects as reaction times are shortened.

Table 2.5 Two Sample t-test p-values Corresponding to the Effects of Quenching in Acetone vs. Air for Three Sets of CdSe Core Synthesis Reactions.

\begin{tabular}{|c|c|c|c|c|c|c|}
\hline $\begin{array}{c}\text { Temp } \\
\left({ }^{\circ} \mathrm{C}\right)\end{array}$ & $\begin{array}{c}\text { Time } \\
(\mathrm{s})\end{array}$ & $\begin{array}{c}\text { Samples } \\
\text { in } \\
\text { Acetone }\end{array}$ & $\begin{array}{c}\text { Samples } \\
\text { in Air }\end{array}$ & $\begin{array}{c}\text { CMW } \\
\text { p-value }\end{array}$ & $\begin{array}{c}\text { Intensity } \\
\text { p-value }\end{array}$ & $\begin{array}{c}\text { FWHM } \\
\text { p-value }\end{array}$ \\
\hline 215 & 30 & 3 & 5 & 0.36 & 0.01 & 0.29 \\
\hline 215 & 60 & 5 & 5 & 0.56 & 0.08 & 0.14 \\
\hline 225 & 60 & 3 & 3 & 0.15 & 0.94 & 0.06 \\
\hline
\end{tabular}

\subsubsection{Temporal Wavelength Shifts in White Fluorescing CdSe Cores}

Reaction times for the synthesis of white fluorescing cores that varied between 0 and 15 seconds still produced samples that fluoresced with white light, however, the spectrums of each sample varied. The more time that passed between injection and extraction the more prominent the primary peak. Figure 2.8 illustrates this effect using two representative samples of WFCs synthesized at 0 and $15 \mathrm{~s}$. A pattern was observed, but not quantitatively tested, indicating a possible link between higher temperatures at the time of injection, combined with immediate removal of small samples and rapid quenching, increasing the presence of the 500nm peaks relative to the other contributing wavelengths. This qualitatively observed alteration produced a solution that was a pale grey blue color. 


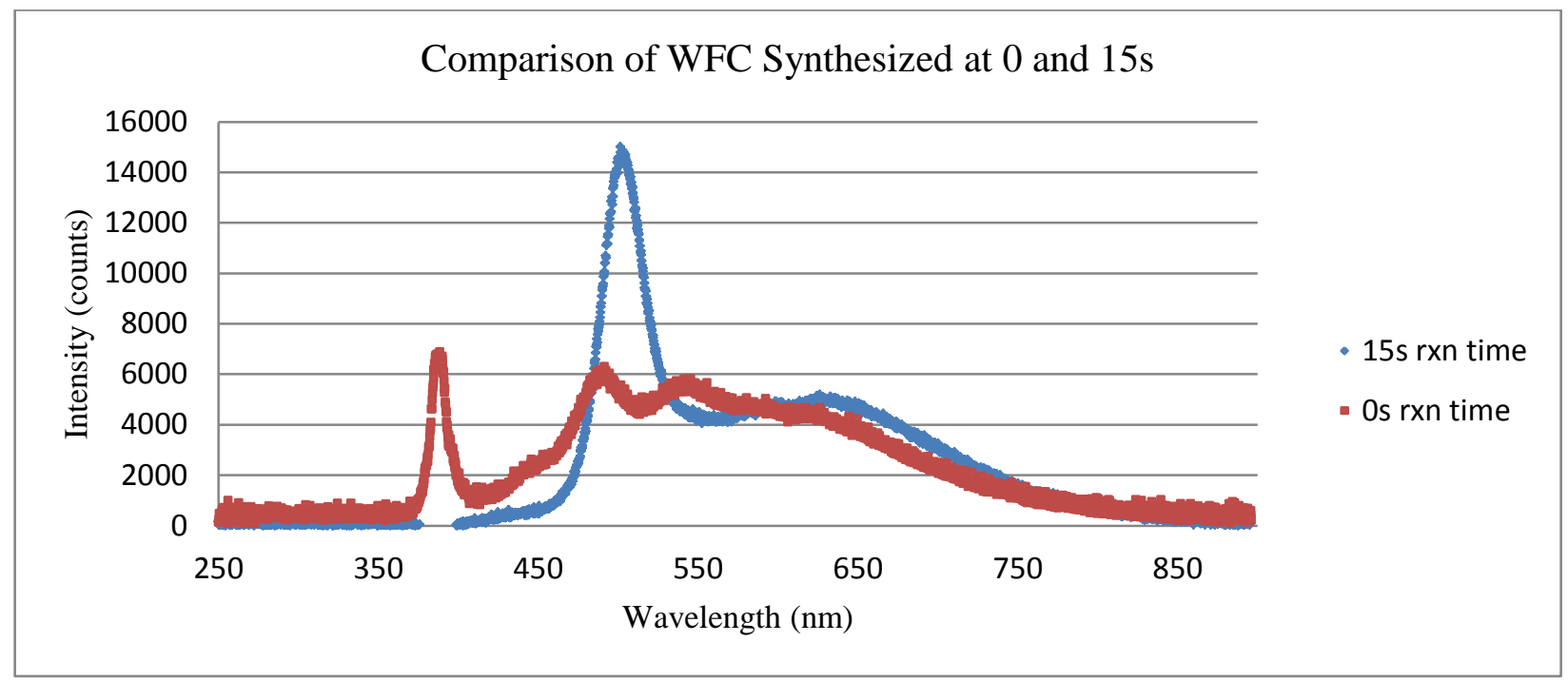

Figure 2.8 Typical fluorescence emission spectra for WFCs synthesized at reaction times of 0 and $15 \mathrm{~s}$.

It was found that over time the majority of WFCs shifted in fluorescence from a crisp white to a neon peach. The transition is quantitatively illustrated by the shift in peak position, shape and intensity. The fluorescence spectrums taken on the day of synthesis, Figure 2.9, show the two typical spectrum shapes associated with WFCs: a primary peak at $475 \mathrm{~nm}$ with a wide rolling tail from 500 to $700 \mathrm{~nm}$ (blue to green visible light spectrum range), and a large non-descript series of wide rounded hills (orange to red visible light spectrum range). The fluorescence spectrum of the same samples, taken 23 days later and show in Figure 2.10, show a significant increase in the primary peaks at $475 \mathrm{~nm}$, as well as the development of a second peak between 620 and $630 \mathrm{~nm}$. It is the development of this second peak in the range of the visible spectrum associated with red, 620 $-750 \mathrm{~nm}$, in addition to the $475 \mathrm{~nm}$ peak associated with blue and cyan, $450-495 \mathrm{~nm}$, that produces the orange appearance of the CdSe solution. The degree of the shift is better illustrated in figure 2.11 where the spectrums for both day 1 and day 23 have been overlapped. 


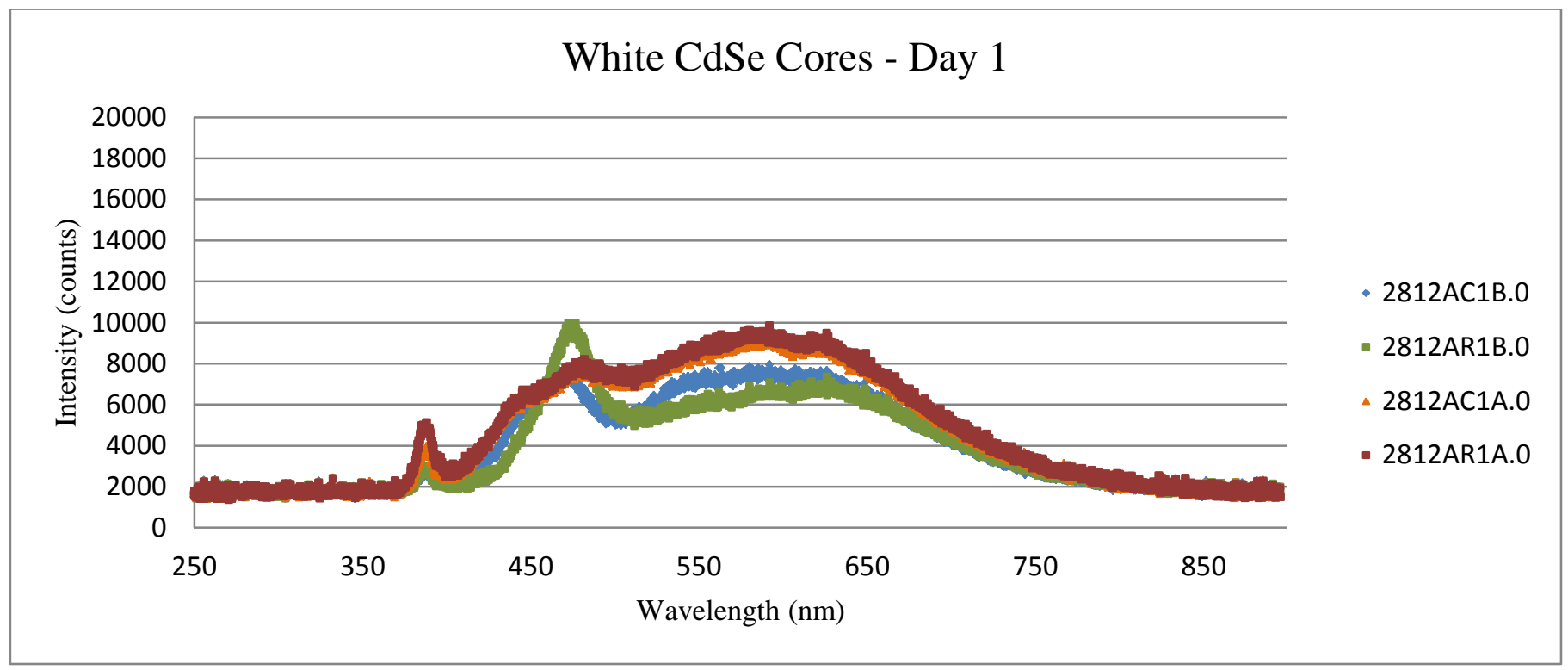

Figure 2.9 Fluorescence spectra of four different WFC solutions taken on the day of synthesis.

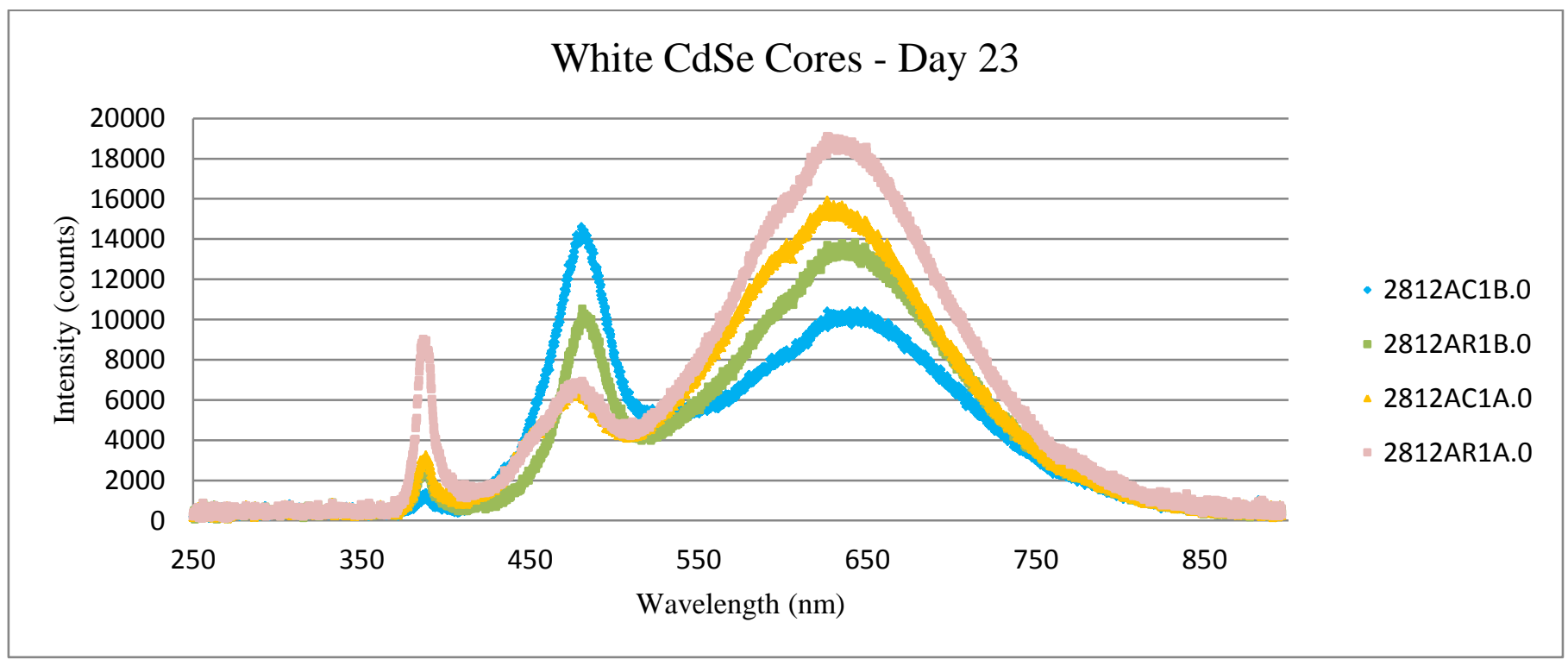

Figure 2.10 Fluorescence spectra of four different WFC solutions taken 23 days after synthesis. 


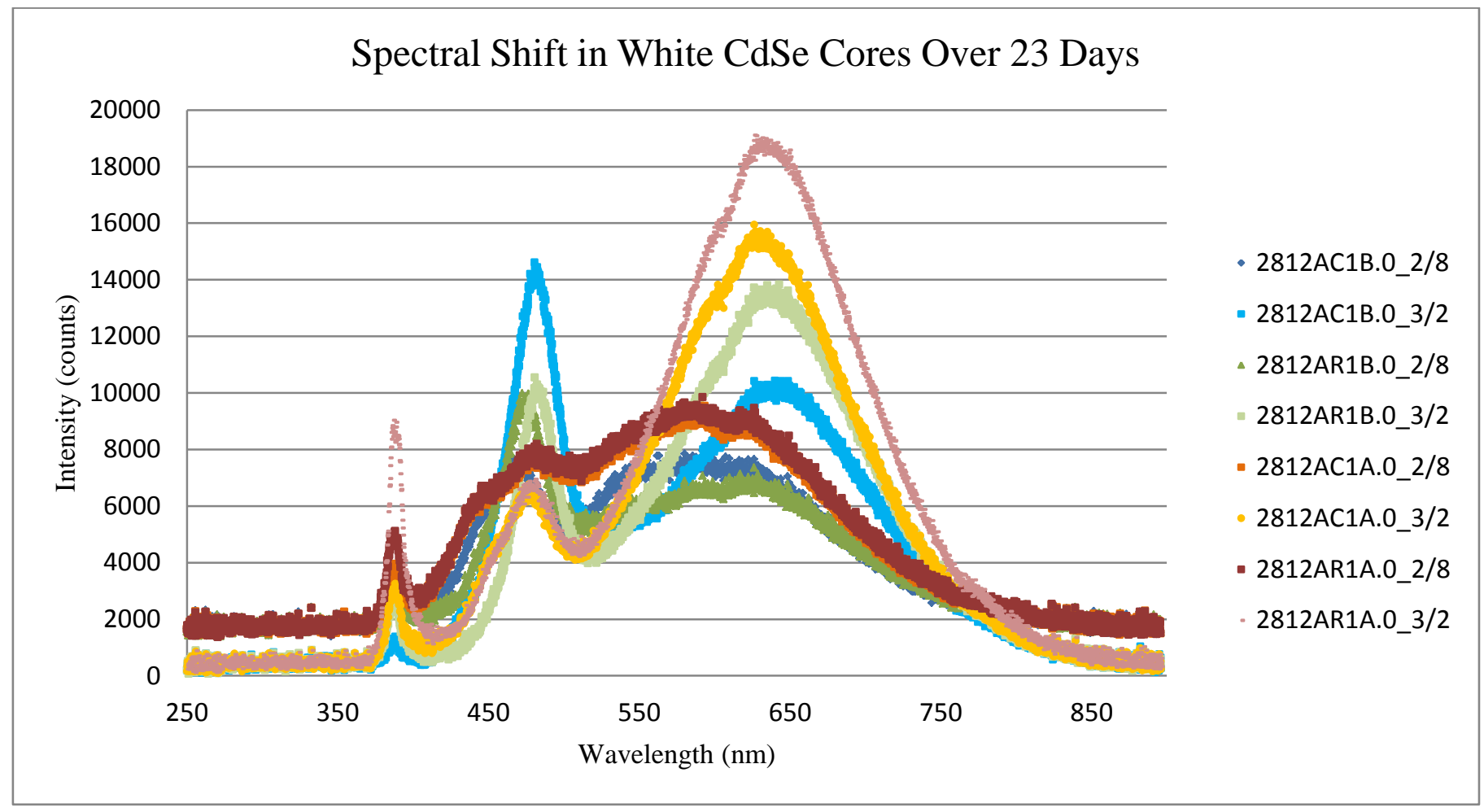

Figure 2.11 Series of four WFC samples synthesized on $2 / 8$, the fluorescence spectrum taken, and then taken again 23 days later on $3 / 2$. The initial spectrum of each sample is in a dark color and the corresponding 23 day time point in a lighter shade of the same color.

\subsubsection{Synthesis of ZnS Coated Quantum Dots}

\subsubsection{ZnS Coating Synthesis Temperature Variation}

In the first round of trials the effect of the $\mathrm{CdSe} / \mathrm{ZnS}$ synthesis temperature on CMW, intensity, and FWHM was investigated by applying a synthesis temperature of $70^{\circ} \mathrm{C}$ and comparing the results to those of the standard protocol synthesis temperature of $160^{\circ} \mathrm{C}$. A two sample t-test, assuming unequal variances, was performed using an alpha of 0.05 to determine if there was a statistically significant difference between the means of the CMW, intensity, and FWHM as a result of the $\mathrm{CdSe} / \mathrm{ZnS}$ synthesis temperature. Table 2.6 shows the fluorescence emission values for each sample with the corresponding averages, standard deviations and p-values given in Table 2.7. A bar chart showing a direct comparison of the average CMWs, intensities, and FWHMs of each synthesis temperature are given in Figure 2.12 with the error bars for each bar that of the 
corresponding standard deviation. The p-values calculated for the CMW and intensity, both 0.004 , support the conclusion that decreasing the $\mathrm{CdSe} / \mathrm{ZnS}$ synthesis temperature of WFCs from $160^{\circ} \mathrm{C}$ to $70^{\circ} \mathrm{C}$ has a statistically significant difference on the final CMW and intensity values. The associated p-value of the FWHM, 0.255, indicates that the null hypothesis cannot be rejected; it supports the conclusion that $\mathrm{CdSe} / \mathrm{ZnS}$ synthesis temperature does not affect the final FWHM of WFCs.

Table 2.6 CMW, Intensity, and FWHM Values for WFCs Coated at $70^{\circ} \mathrm{C}$ and $160^{\circ} \mathrm{C}$.

\begin{tabular}{|l|c|c|c|c|}
\hline \multicolumn{1}{|c|}{ Sample } & $\begin{array}{c}\text { Temp } \\
\left({ }^{\circ} \mathrm{C}\right)\end{array}$ & $\begin{array}{c}\text { CMW } \\
(\mathrm{nm})\end{array}$ & $\begin{array}{c}\text { Intensity } \\
(\text { counts })\end{array}$ & $\begin{array}{c}\text { FWHM } \\
(\mathrm{nm})\end{array}$ \\
\hline 32512QD.30 & 70 & 522 & 36000 & 32 \\
\hline 32512AC.15 & 70 & 505 & 49000 & 31 \\
\hline 41012wQD.0a & 70 & 482 & 51000 & 36 \\
\hline 32412QD.15B & 70 & 515 & 42000 & 34 \\
\hline 32412QD.15A & 160 & 569 & 7400 & 30 \\
\hline 42812gQD.1a & 160 & 560 & 3600 & 37 \\
\hline 41012wQD.0b & 160 & 550 & 7300 & 39 \\
\hline 42612wQD.2a & 160 & 547 & 3000 & 41 \\
\hline
\end{tabular}

Table 2.7 Averages and Standard Deviations of CMW, Intensities, and FWHMS of WFCs Coated at $70^{\circ} \mathrm{C}$ and $160^{\circ} \mathrm{C}$.

\begin{tabular}{|c|c|c|c|c|}
\hline & $\begin{array}{c}\text { Temp } \\
\left({ }^{\circ} \mathrm{C}\right)\end{array}$ & $\begin{array}{c}\text { CMW } \\
(\mathrm{nm})\end{array}$ & $\begin{array}{c}\text { Intensity } \\
(\text { counts })\end{array}$ & $\begin{array}{c}\text { FWHM } \\
(\mathrm{nm})\end{array}$ \\
\hline avg & 70 & 506 & 44500 & 33 \\
\hline st. dev. & 70 & 17 & 6856 & 2 \\
\hline avg & 160 & 557 & 5325 & 37 \\
\hline st. dev. & 160 & 10 & 2351 & 5 \\
\hline p-value & & 0.004 & 0.004 & 0.255 \\
\hline
\end{tabular}




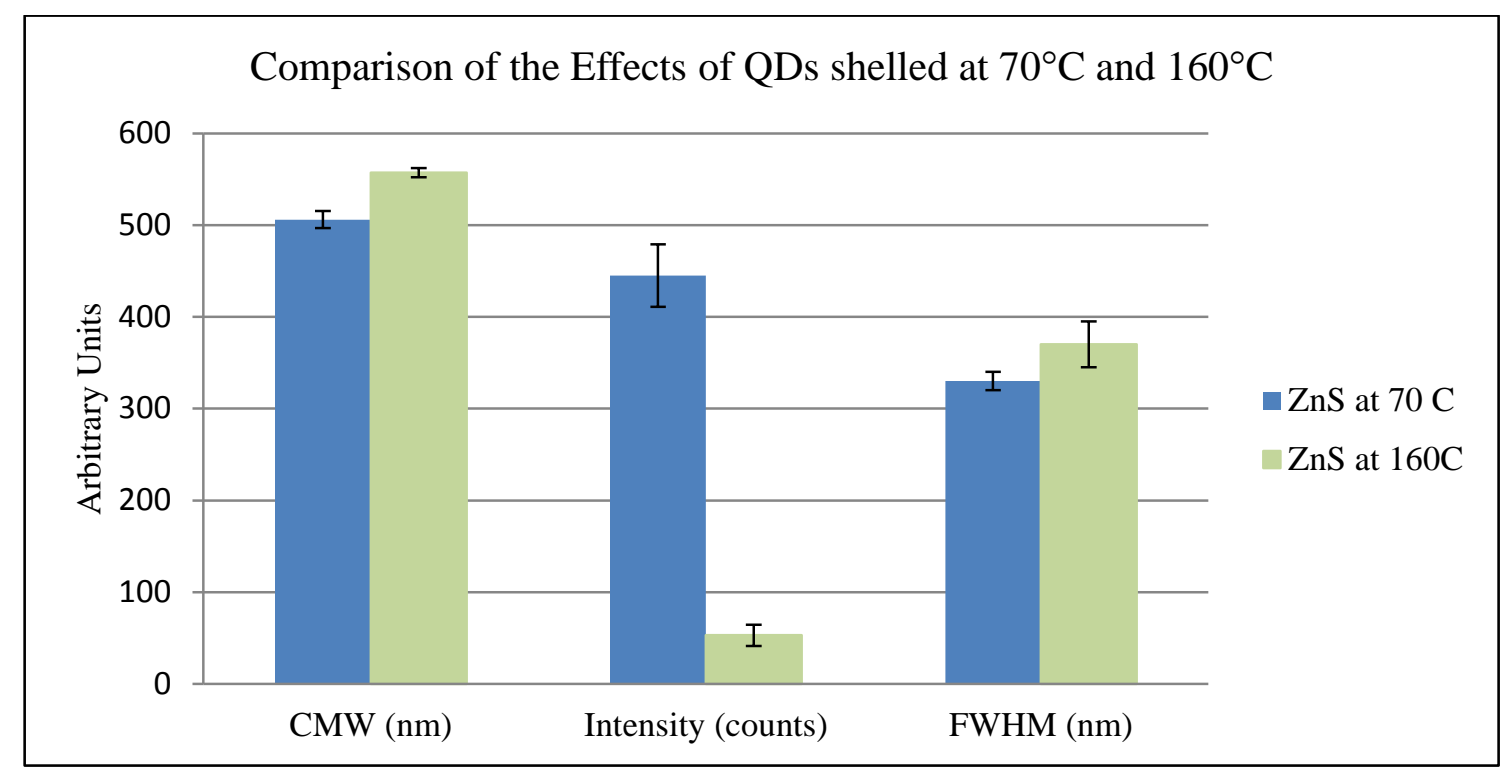

Figure 2.12 Comparison of the average CMWs, Intensities, and FWHMs of each synthesis temperature with the error bars for each corresponding to the associated standard deviation.

In the second round of trials the effect of the $\mathrm{CdSe} / \mathrm{ZnS}$ synthesis temperature on $\mathrm{CMW}$ was investigated by applying a synthesis temperature of $110^{\circ} \mathrm{C}$ in addition to the standard protocol synthesis temperature of $160^{\circ} \mathrm{C}$. As a result of each sample set only containing 2 samples for each temperature, only qualitative methods were applied for the assessment of the QD end products synthesized at $110^{\circ} \mathrm{C}$ and $160^{\circ} \mathrm{C}$. A visual comparison the fluorescence emission produced by the QDs synthesized at $110^{\circ} \mathrm{C}$ and $160^{\circ} \mathrm{C}$ of each sample set, shown in Figure 2.13 , was used as a qualitative assessment as to the effect of each coating temperature. In both sample sets, white and $541 \mathrm{~nm}$, it was concluded that lower coating temperatures produced QDs with shorter wavelengths. 

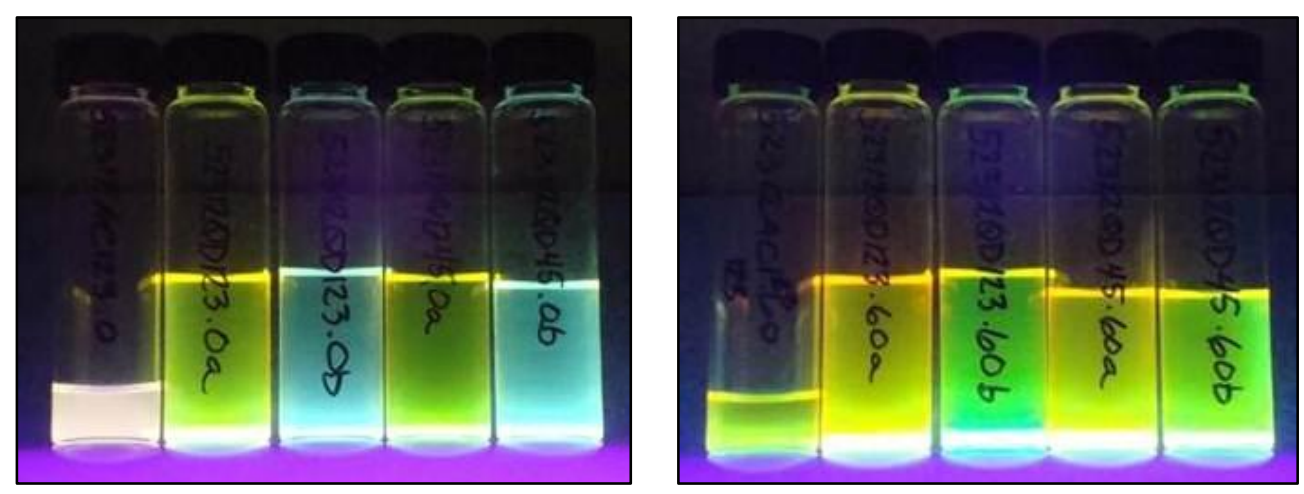

Figure 2.13 Qualitative comparison of $\mathrm{ZnS}$ shelling temperatures. Left: white fluorescing starting $\mathrm{CdSe}, 0 \mathrm{a}$ shelled at $160^{\circ} \mathrm{C}, 0 \mathrm{~b}$ shelled at $110^{\circ} \mathrm{C}$. Right: green fluorescing starting CdSe, 60a shelled at $160^{\circ} \mathrm{C}, 60 \mathrm{~b}$ shelled at $110^{\circ} \mathrm{C}$.

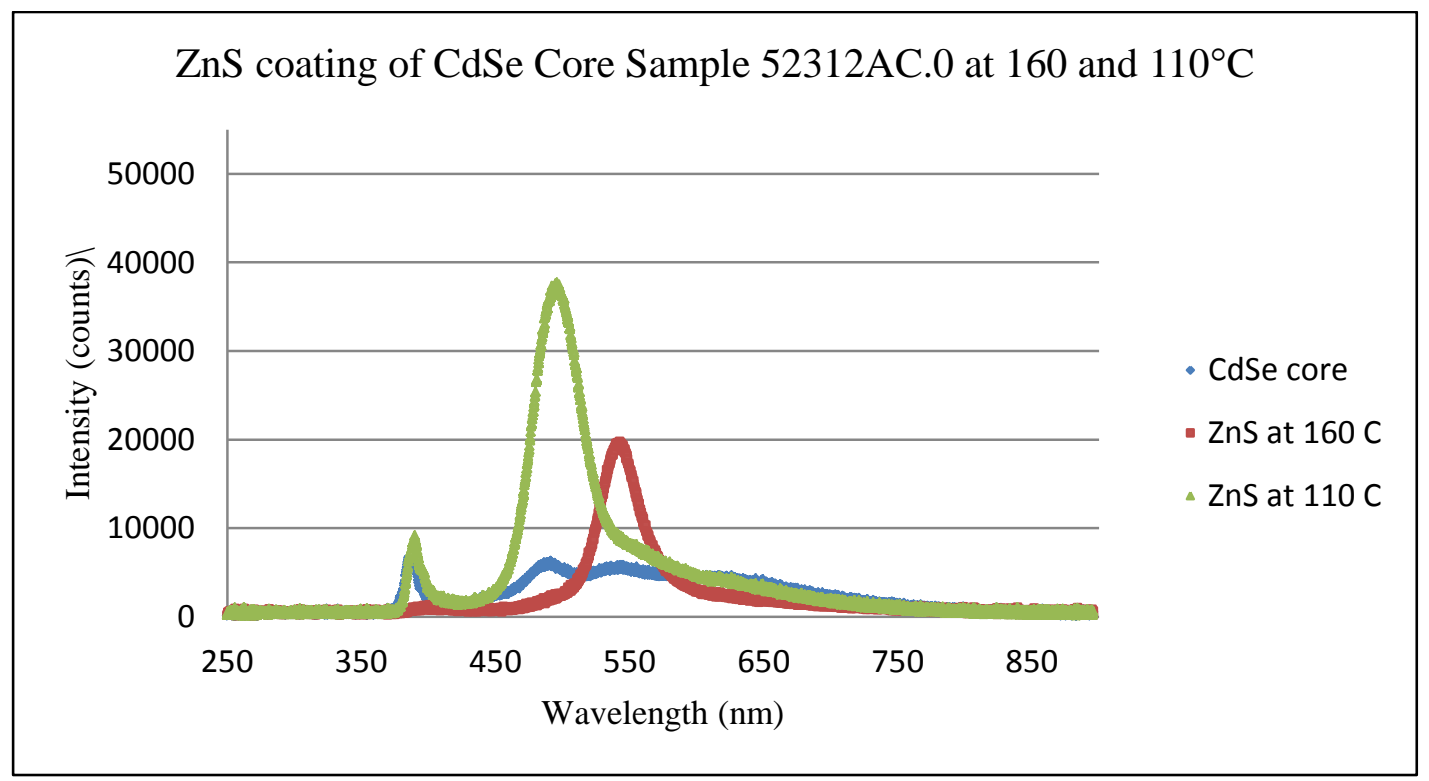

Figure 2.14 Averaged fluorescence spectrum of white fluorescing starting $\mathrm{CdSe}, 160^{\circ} \mathrm{C}(\mathrm{CMW}$ : 540nm; intensity: 19500 counts; FHWM: 39nm), and $110^{\circ} \mathrm{C}$ (CMW: 495nm; intensity: 37500 counts; FHWM: 43nm), ZnS shelling temperatures. 


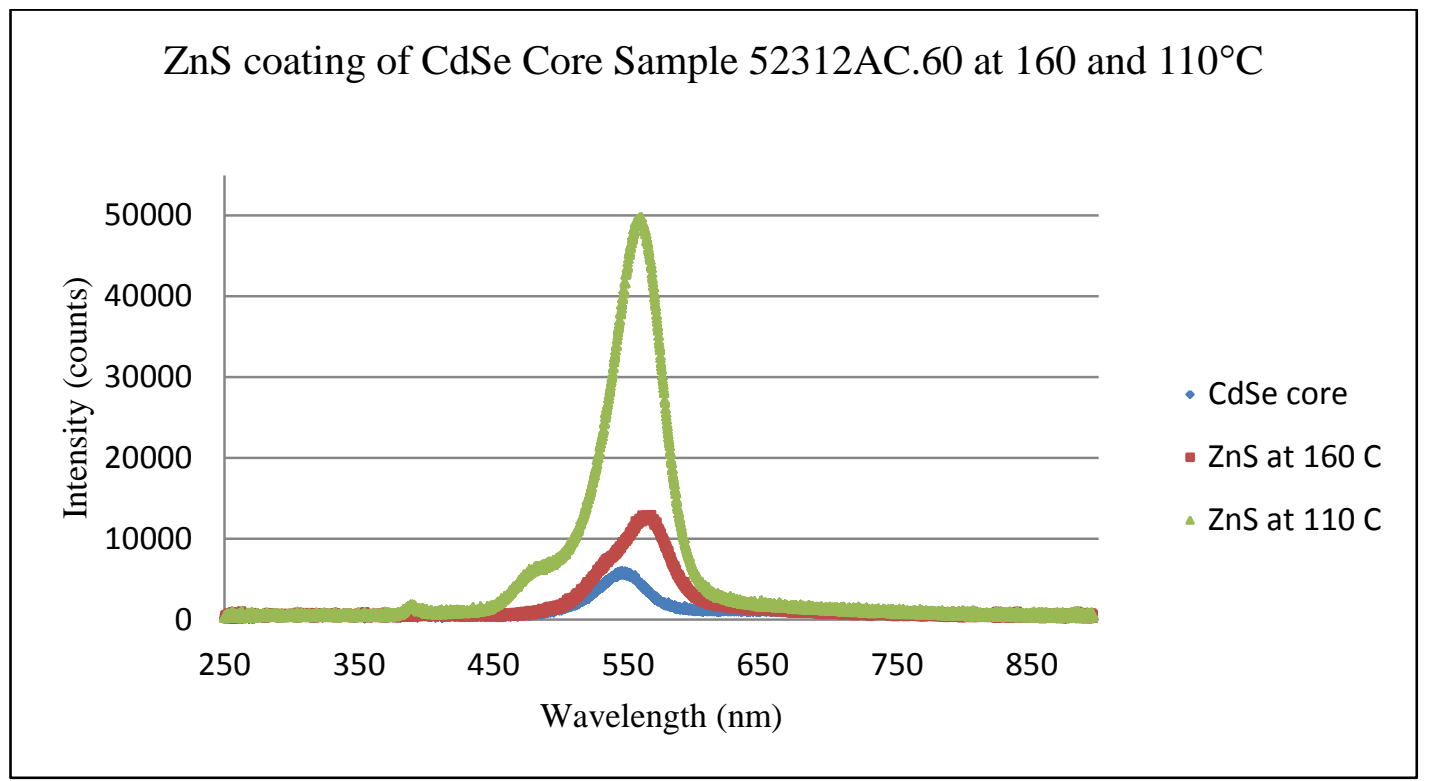

Figure 2.15 Averaged fluorescence spectrum of green fluorescing starting CdSe (CMW: 541nm; intensity: 5800 counts; FHWM: 44nm, $160^{\circ} \mathrm{C}$ (CMW: 560nm; intensity: 12900 counts; FHWM:

54nm), and $110^{\circ} \mathrm{C}$ (CMW: 555nm; intensity: 49000 counts; FHWM: 46nm), ZnS shelling temperatures.

\subsection{Discussion}

\subsubsection{Synthesis of CdSe Cores}

Transference of the dry reagents directly into the $50 \mathrm{~mL}$ round bottom flask, by-passing the use of the plastic weigh-boats, was done to reduce the discrepancy between the amount of powder weighed out and the amount that made it into the flask. The selenium powder had a tendency to adhere to the plastic and the cadmium oxide powder, as a result of static electricity, tended to bounce out of the weigh-boat during transferring. It was found that quick and precise movements, on the part of the chemist, during the transfer of the powders from their regent containers to the reaction flasks, made using the weigh-boats unnecessary.

During the many CdSe core synthesis procedures Se-TOP precursor solutions of different ages, ranging from 1 to 14 days, were used. After many applications of the different ages of the Se-TOP precursors, all in conjunction with the use of fresh Cd precursor, it was concluded that Se- 
TOP solutions were best used within five days of their synthesis and would consistently give poor results if used more than 10 days after their initial synthesis. Unlike the Se-TOP precursor, the Cd precursor returned better results when made in batches no bigger than twice the amount of the starting values.

\subsubsection{Experimental Variables Investigated for the General Synthesis of CdSe Cores}

A potential method for investigating volume-related effects, in an effort to synthesize CdSe cores within the $450-500 \mathrm{~nm}$ range, would be to implement the use of long thin glass tubing as a receptacle for which to inject the sample. This tubing could then be submerged in an ice bath which, along with its reduced diameter, would increase the rate of heat extraction from the sample. Manipulation of the temperature at the time of the Se-TOP injection was initially thought to be a possible mechanism for fine-tuning the CMW of the CdSe cores. Although the data would indicate otherwise, it still may be a parameter to be further investigated for CdSe synthesis involving reaction time points of longer than 90 s. Additionally synthesis temperatures higher than $225^{\circ} \mathrm{C}$ or lower than $215^{\circ} \mathrm{C}$ may show a statistically significant effect on the CMW, intensity, and FWHM of CdSe cores.

A change in emission wavelength relative to changes in the size of the QD is an inherent characteristic readily accepted in the synthesis of QDs. Likewise duration of synthesis is related to QD size and therefore QD emission wavelength. The purpose of testing the effect of different reaction time points was not to reinvent the wheel, but rather to quantitatively determine the effect of reaction times of small 30 second increments on CMW, intensity, and FWHM. The data supported the conclusion that reaction times have a logarithmic effect on the CMW of CdSe cores; the greatest change in CdSe emission wavelength is seen within the first two minutes. Further experimentation investigating which variable, reaction temperature or reaction time, is more influential at longer time points should be explored. 


\subsubsection{Synthesis of White Fluorescing CdSe Cores}

The process for synthesizing of WFCs was stumbled upon while attempting to synthesize blue QDs. It was reasoned early on in the CdSe core synthesis experiments that if the duration of the reaction led to $\mathrm{CdSe}$ cores of increased diameter, and therefore an increased emission wavelength, that a reduction in the duration of the reaction would have the opposite effect. The initial motivation was to produce the ever elusive blue emission wavelength; the result was a range of wavelengths in s single sample, creating the emission of white light. As a result of the peaks present in the fluorescence spectrums corresponding to the 15 s time points, it is postulated that if a size selection filtration of the $15 \mathrm{~s}$ WFCs was employed it may be possible to acquire a solution consisting of QDs emitting in the $475-500 \mathrm{~nm}$ range in the visible spectrum associated with blue light.

\subsubsection{Temporal Wavelength Shifts in White Fluorescing CdSe Cores}

The phenomenon of spectral shift frequently seen in the WFCs, and in one case WFCs that had been $\mathrm{ZnS}$ coated and still initially maintained their white emission, appears to be a result of the lowered synthesis temperature in both the CdSe core and $\mathrm{ZnS}$ coating procedures. Although spectral shifts of $\mathrm{ZnS}$ coated WFCs were not measured in the data presented, a visual comparison of several samples indicated a similar shift. The shift in CdSe cores was seen in samples synthesized below $215^{\circ} \mathrm{C}$; the shift was observed in the WFCs coated with $\mathrm{ZnS}$ using synthesis temperatures below $70^{\circ} \mathrm{C}$

\subsubsection{Synthesis of ZnS Coated Quantum Dots}

Using a standard ratio of $5 \mathrm{~mL}$ of $\mathrm{CdSe}$ core solution to $1 \mathrm{~mL} \mathrm{ZnS}$ precursor for use in every shelling procedure introduces unknown variability. In Josh Angell's thesis the ratios chosen were dependent on the average diameter calculated for the CdSe cores. This same approach was not applied and therefore may have contributed to the inconsistencies seen in the intensities of the QD 
emission peaks. Although no quantitative data was provided, it was noted during ZnS synthesis trials that WFC samples tended to show better results using the standard 5 to $1 \mathrm{~mL}$ CdSe core to $\mathrm{ZnS}$ precursor.

\subsubsection{ZnS Coating Synthesis Temperature Variation}

The purpose for pursuing lower $\mathrm{ZnS}$ coating temperatures, such $70^{\circ} \mathrm{C}$ and $110^{\circ} \mathrm{C}$, had been to test the hypothesis that a gradient of $\mathrm{ZnS}$ coating temperatures could be used as an alternative method for achieving QDs with smaller emission wavelengths shifts. It was discovered that this was in fact true; lower coating temperatures did result in smaller shifts in CMW. An additional side-effect, quantitatively supported, was an increase in intensity when these lower ZnS synthesis temperatures were applied to WFCs. A qualitative assessment of the fluorescence emission spectra in Figure 2.14 leads to the conclusion that it is very likely that lower $\mathrm{ZnS}$ synthesis temperatures may affect all CdSe solutions in the same way; lowering their shift in CMW and increasing their intensity.

A straight forward, albeit generic method, that could be employed in future experiments, with respect to $\mathrm{CdSe}$ to $\mathrm{ZnS}$ ratios during the shelling process, would be to adjust only the amount of $\mathrm{ZnS}$ precursor injected for each sample of CdSe core. Applying this approach to several samples, categorizing the CdSe cores based on their emission wavelength, would be a first step in experimental determining the relationship between $\mathrm{CdSe}$ and $\mathrm{ZnS}$ ratios. An additional approach would be to use WFC as the initial sample and apply the primary parameter, reaction time, associated with the WFC synthesis procedure as a second variable. 


\section{CHAPTER 3 - Quantum Dot Construct Synthesis}

\subsection{Introduction}

The emission characteristics associated with QDs make them an attractive tool for imaging in biologic mediums. The tunable quality of the emission wavelengths of QDs, along with their resistance to photo-bleaching, makes them a valuable device for use as biologic tags over their organic counterparts. An added benefit to using QDs over organic fluorophores is the wide range of excitation wavelengths that can be used to excite multiple QDs, making it possible to use a single source to simultaneously excite a variety of QDs. All of these advantageous qualities however, are useless for application in biologic mediums if the QDs remain hydrophobic. For QDs to be available for use in biologic applications they must first be made water soluble. There are multiple methods for achieving this including bioconjugation, ligand exchange, and polymer wrapping. The method investigated in this thesis was the encapsulation of the hydrophobic QDs inside of a hydrophilic phospholipid micelle. Phospholipids were the method of QD encapsulation chosen in light of the fact that the ultimate goal was to introduce the solubilized QDs into EpiDerm skin substitutes. The introduction of the QDs would be done by applying methods developed by Russell et.al. pertaining to the interaction of phospholipids within the skin substitutes. It is hoped that the following encapsulation method will be applied in future endeavors involving the EpiDerm skin substitutes.

\subsection{Methods}

\subsubsection{QD Precipitation}

After the procedure for coating the CdSe cores in a $\mathrm{ZnS}$ shell was completed, the QDs were left suspended in octadecane. Octadecane (ODE) is a straight chain molecule of the alkane family 
with an 18 carbon backbone. Alkanes are insoluble in polar solvents such as water, PBS, or cell and tissue culturing media; alkanes are hydrophobic (75). As part of the alkane family of hydrocarbons, ODE is also hydrophobic. This quality makes it an excellent solvent for the QDs but a poor solvent for phospholipids. Phospholipids are, however, readily soluble in organic solvents such as chloroform (76). As such, the QDs needed to be suspended in chloroform before their introduction to the phospholipids. This was accomplished by precipitation of the QDs out of the ODE and then resuspension of the QDs into chloroform. The method used for the precipitation of the QDs was developed by Tommy Garting and utilized centrifugation and ethanol in concert (77). Ethanol (EtOH) acts as an anti-solvent, a medium in which the QDs are insoluble. This along with ethanol's characteristic density of $0.790 \mathrm{~g} / \mathrm{mL}$, greater than ODE's density of $0.777 \mathrm{~g} / \mathrm{mL}$, removed the QDs from the ODE and created a pellet of QDs in the tip of the centrifuge tube (78). Typically two centrifuge tubes were used, into each were placed $3 \mathrm{~mL}$ of CdSe/ZnS QDs suspended in ODE. If a large QD sample, greater than $6 \mathrm{~mL}$, was to be precipitated then the sample was uniformly distributed across an even number of centrifuge tubes. Previous precipitation methods used a single tube containing a QD sample that was then balanced in the centrifuge by a tube containing a volume of water equal in weight to the volume of the QD sample. The precipitation process consisted of adding to the centrifuge tube containing the QDs, a volume of EtOH of at least $3 \mathrm{~mL}$, but no more than twice that relative to the volume of the QD contents. The addition of the EtOH caused the contents of the tube to develop an oil-in-water appearance as a result of the immiscibility of the ODE and the EthOH; Figure 3.1A illustrates this condition. After addition of the ethanol the centrifuge tube was capped and shaken. The solution was centrifuged for a length of 10 minutes at a rate of 4000 RPM. After centrifugation the supernatant was removed. Removal of the supernatant was done using a 3mL syringe and needle and removing the uppermost portion first. A method of removal working from the top down was performed to ensure 
the complete removal of the ODE. As a result of ODE having a lower density than EtOH, it would inevitably remain in the top layer of the supernatant. Removal of the supernatant from the lower portion of the liquid results in a residue of ODE being left behind and greatly hinders the complete evaporation and quality of the precipitate. Repeated addition of ethanol, centrifugation, and removal of the subsequent QD-free supernatant with a 3mL syringe, resulted in the eventual isolation of the QDs in the tip of the centrifuge tube as shown in Figure 3.1B. Each volume of removed supernatant was expelled into the same waste vial. The vial was then held up to a black light to determine if any QDs had been inadvertently removed as shown in Figure 3.1C. If the vial was positive for the presence of QDs, the waste solution was divided equally into centrifuge tubes and spun down for a secondary precipitation of the QDs. Once the QDs appeared to be firmly set in the tip of the tube, the tubes were inverted and the viscosity of the QD precipitate was used as a qualitative assessment as to the successful removal of all ODE. If there was any movement of the precipitate out of the tip of the centrifuge tube, an additional set of the previously cited precipitation steps was conducted. After isolation, the centrifuge tubes containing the precipitated QDs were left in the fume hood for a minimum of 24 hours to ensure that the QD precipitate was dry and free from any residual solvent before their re-suspension in chloroform. Occasionally a black precipitate would form in the tip of the tube. This black precipitate was carefully removed. The entire step by step protocol for the precipitation of QDs out of ODE is given in Appendix B.4. 


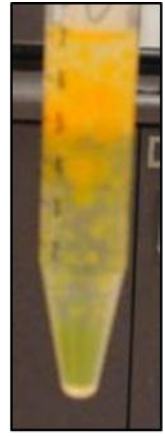

A

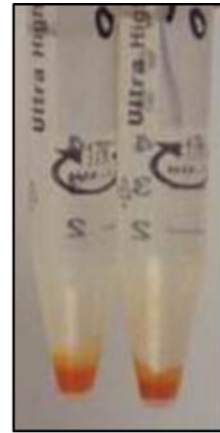

B

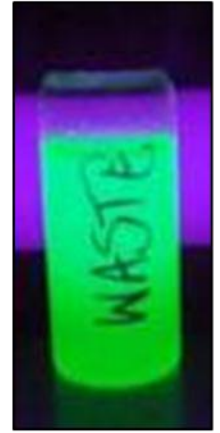

C

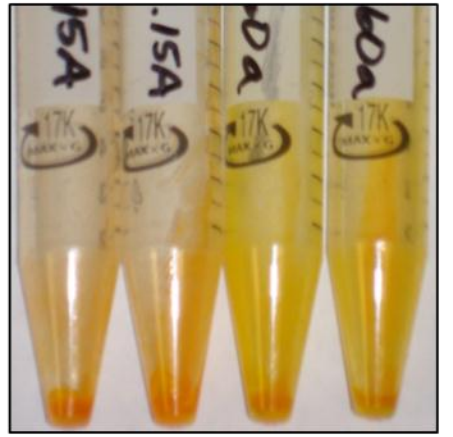

D

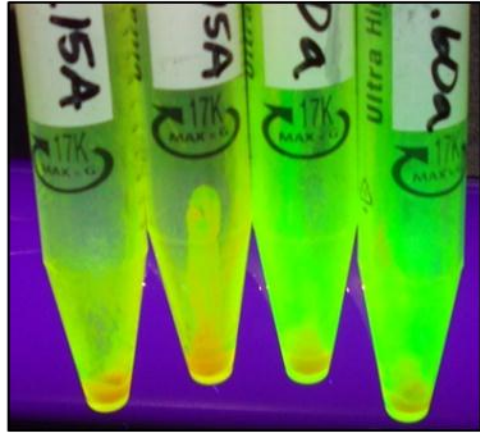

$\mathbf{E}$

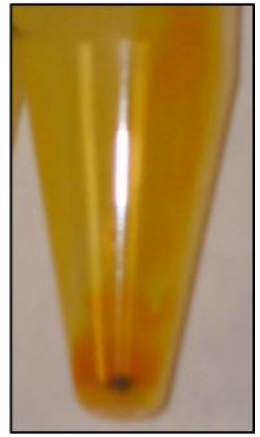

$\mathbf{F}$

Figure 3.1 A visual representation of the steps involved in precipitation: A - QDs in ODE with EtOH added; B - precipitated QDs with supernatant; C - QDs in supernatant waste; D precipitated QDs; E - precipitated QDs under black light; F- black precipitate.

\subsubsection{QD Suspension into $\mathrm{CHCl}_{3}$}

After the QD precipitate had a minimum of 24 hours in the fume hood the QD precipitate was inspected for consistency of color. Occasionally a small particle of black precipitate was detected in the tip. This black particulate was attributed to precipitation of selenium out of the core CdSe composite. Into each centrifuge tube $1.5 \mathrm{~mL}$ of chloroform was added. This resulted in a total of 3mL of suspended QDs. The suspension was then visually inspected for signs of saturation. If saturation was apparent, QDs were precipitating out of the chloroform, an additional amount of chloroform was added in increments of $0.5 \mathrm{~mL}$ until a consistent solution of suspended QDs was achieved.

\subsubsection{Phospholipid Concentration, Suspension, and Encapsulation}

\subsubsection{Experimental Trials - Round One and Two}

Multiple steps were involved in creating phospholipid encapsulated QDs that could be suspended in an aqueous media. The course of action taken was to determine an appropriate phospholipid concentration, an optimal phospholipid to QD ratio, and a successful method of evaporation that would remove the solvent and suspend the phospholipid encapsulated QDs into the aqueous media. The saturation tolerance for dissolving phospholipids in chloroform is much 
higher than the saturation tolerance of PBS or keratinocyte media. This fact had to be taken into account when choosing a PL to solvent ratio in the initial step that would yield the desired PL concentration. The appropriate PL to solvent ratio was determined by designing a series of experiments that tested the effects of all of the parameters involved in the encapsulationevaporation process on raising or lowering the saturation points of the PL in each solvent. The solvent, PL concentration within the solvent, ratio of PL to QDs, ratio of suspended PL/QDs with respect to the solution they were to be evaporated into, the application of heat to the mixture, enclosing the reaction, and the use of agitating the mixture were all tested. A sample arrangement of the parameters tested, using $2.5 \mathrm{mg} / \mathrm{mL}$ as the PL concentration, is given in Table 3.1. This arrangement of tests was duplicated using the additional concentrations of PL in solvent of 1, 5, and $10 \mathrm{mg} / \mathrm{mL}$.

Table 3.1 PL Concentration and Ratio Test Parameters Applied with 2.5mg/mL Trial

\begin{tabular}{|c|c|c|c|c|}
\hline Trial & $\begin{array}{c}\text { PL/solvent } \\
(\mathrm{mg} / \mathrm{mL})\end{array}$ & solvent & $\begin{array}{c}\text { QD/CHCl3 } \\
(\mathrm{mL})\end{array}$ & $\begin{array}{c}\text { PL/CHCl3 } \\
(\mathrm{mL})\end{array}$ \\
\hline $\mathrm{A}$ & 2.5 & $\mathrm{PBS}$ & 1 & 3 \\
\hline $\mathrm{A}$ & 2.5 & $\mathrm{PBS}$ & 1 & 2 \\
\hline $\mathrm{A}$ & 2.5 & $\mathrm{PBS}$ & 1 & 1 \\
\hline $\mathrm{B}$ & 2.5 & $\mathrm{CHCl} 3$ & 1 & 3 \\
\hline $\mathrm{B}$ & 2.5 & $\mathrm{CHCl} 3$ & 1 & 2 \\
\hline $\mathrm{B}$ & 2.5 & $\mathrm{CHCl} 3$ & 1 & 1 \\
\hline $\mathrm{C}$ & 2.5 & $\mathrm{CHCl} 3$ & 1 & 3 \\
\hline $\mathrm{C}$ & 2.5 & $\mathrm{CHCl} 3$ & 1 & 2 \\
\hline $\mathrm{C}$ & 2.5 & $\mathrm{CHCl} 3$ & 1 & 1 \\
\hline $\mathrm{D}$ & 2.5 & $\mathrm{CHCl} 3$ & 1 & 3 \\
\hline $\mathrm{D}$ & 2.5 & $\mathrm{CHCl} 3$ & 1 & 2 \\
\hline $\mathrm{D}$ & 2.5 & $\mathrm{CHCl} 3$ & 1 & 1 \\
\hline
\end{tabular}

During the initial test trials each variation of the solutions containing phospholipids were investigated for possible contributions of the phospholipids to the fluorescence spectrum as a result of auto-fluorescence. Solutions of phospholipids at multiple concentrations and in multiple 
solvents, such as $\mathrm{CHCl}_{3}, \mathrm{CHCl}_{3}: \mathrm{MeOH}$, keratinocyte media at different filtration levels, and PBS

were investigated. Additionally the fluorescence spectrums of QDs, before and after the

introduction of the phospholipids, were also generated as a method for assessing the effect that the phospholipids had on the CMW and FWHM of the test solutions.

Four arrangements of the evaporation test parameters were then designed using each of the three different ratios of QDs to PLs. with a PL concentration of $2.5 \mathrm{mg} / \mathrm{mL}$ as shown in Table 3.2.The four different sets of evaporation steps were labeled A, B, C, and D. Set A used 10mL of a $2.5 \mathrm{mg} / \mathrm{mL}$ solution of PL/PBS in a 1:1, $1: 2$, and $1: 3$ ratio of $\mathrm{QD} / \mathrm{CHCl}_{3}$ : $\mathrm{PL} / \mathrm{PBS}$, evaporated during heating at $60^{\circ} \mathrm{C}$. Set $\mathrm{B}$ used $10 \mathrm{~mL}$ of a $2.5 \mathrm{mg} / \mathrm{mL}$ solution of $\mathrm{PL} / \mathrm{CHCl}_{3}$ in a $1: 1,1: 2$, and 1:3 ratio of $\mathrm{QD} / \mathrm{CHCl}_{3}$ : $\mathrm{PL} / \mathrm{PBS}$, added $2 \mathrm{~mL}$ of $\mathrm{PBS}$, and then evaporated during heating at $60^{\circ} \mathrm{C}$. Set $\mathrm{C}$ used the same concentrations and ratios as set $\mathrm{B}$, but attempted to evaporate the $\mathrm{CHCl}_{3}$ first, then add $2 \mathrm{~mL}$ PBS and heat at $60^{\circ} \mathrm{C}$. Set D also used the same concentrations and ratios as set B, but added the $2 \mathrm{~mL}$ of PBS and attempted to evaporate without heat employing only the air current generated inside the fume hood.

Table 3.2 PL Encapsulation and Evaporation Parameters

\begin{tabular}{|c|l|}
\hline Trial & \\
\hline A & Evaporation during heating at $60^{\circ} \mathrm{C}$ \\
\hline B & Add $2 \mathrm{~mL}$ PBS, evaporation during heating at $60^{\circ} \mathrm{C}$ \\
\hline C & Evaporate, add $2 \mathrm{~mL}$ PBS, heat at $60^{\circ} \mathrm{C}$ \\
\hline D & Add $2 \mathrm{~mL} P B S$, evaporation, NO heat \\
\hline
\end{tabular}

During this time a series of slides were prepared using samples with a variety of QD/PL concentrations that had been suspended in $\mathrm{CHCl}_{3}: \mathrm{MeOH}$, then had $2 \mathrm{~mL}$ of PBS added, heated for a period of 10 minutes at 55C, and then left to evaporate in the hood for 2 days. These slides were then imaged with a confocal microscope, using filter Qdot565, and the images for each concentration qualitatively analyzed. 
A second round of trials was employed specifically to investigate methods for increasing the rate of evaporation of the chloroform and promoting the suspension of the phospholipid encapsulated QDs (QD constructs) into an aqueous media. Among the multiple strategies investigated were the application of nitrogen gas, a magnetic spine vane, closed vials with vent needles versus open vials, and the size of the evaporation container. In all of these tests the vials were exposed to heat by being submerged in a $55^{\circ} \mathrm{C}$ silicon oil bath. Nitrogen gas was applied to test two methods for increasing the evaporation rate: agitation and increased flow over the surface of the solution. The nitrogen needle was placed both below the surface of the solution and $1 \mathrm{~cm}$ above the surface with a flow rate high enough to disturb the solution but not eject it out of the vial. A magnetic spin vane was placed inside of the solution and set to spin at $300 \mathrm{RPM}$ in an effort to expose the higher density chloroform to air flow as well as to continually mix the solution, maintaining an even distribution of QD constructs. As a result of the long duration necessary to achieve of evaporation seen in the first round of encapsulation trials, capping the vials with rubber septums into which vent needles were placed, as shown in Figure 3.3, was investigated. This method employed to promote evaporation of the $\mathrm{CHCl}_{3}$ by increasing the pressure in the vial while maintaining a consistent temperature and reducing the evaporation of the PBS. 


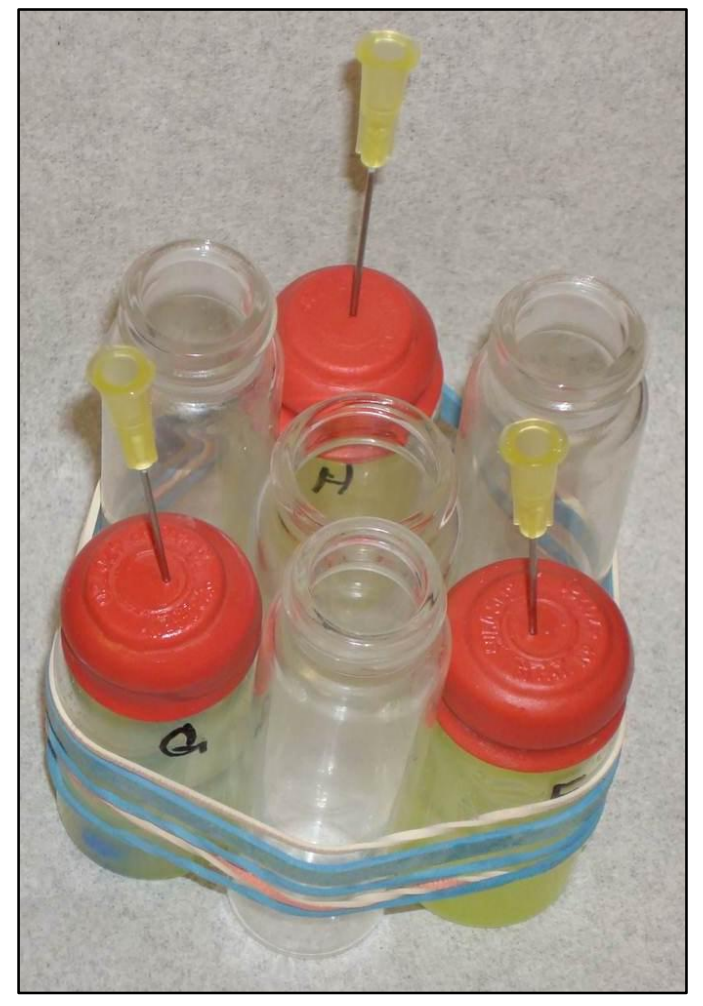

Figure 3.2 Arrangement of three vials containing QD construct solutions with rubber septums and vent needles.

Increasing the size of the evaporation container, to increase the size of the surface area exposed to air flow, was accomplished by placing the test solution into a $50 \mathrm{~mL}$ glass beaker with a $5 \mathrm{~cm}$ diameter. The discovery in chapter 2 , of $\mathrm{CHCl}_{3}$ degrading the plastic cuvettes, was used as an initial determination for the presence of $\mathrm{CHCl}_{3}$ within the evaporating test solution. Although use of the cuvette could not definitively determine the absence of chloroform, placing a cuvette into the solution of interest, and noting its appearance 5 minutes after exposure, could positively confirm the continued presence of chloroform. 


\subsubsection{Final Encapsulation Method}

The final protocol for the phospholipid encapsulation of QDs, and their suspension into an aqueous media via evaporation, was developed by incorporating the factors that were deemed successful from the initial round of trials. These factors were then altered to accommodate the desired PL/keratinocyte media concentration of $10 \mathrm{mg} / \mathrm{mL}$ or $1.0 \% \mathrm{w} / \mathrm{v}$ that was used in the Russell et. al. paper. The first step was choosing a CdSe/ZnS sample, created using the process described in chapter 2, which had an appropriate CMW and FWHM for imaging with fluorescent stains without overlap of the emission spectrums. For round one (R1) of the keratinocyte trials the CdSe/ZnS QD chosen was 42612wQD.2a with a CMW of 551nm and FWHM of 42nm, which provided an emission that fell between the wavelengths emitted by the Calcein and EthD-1 fluorescent stains used for testing cell viability: 515 and $635 \mathrm{~nm}$ respectively. The $10 \mathrm{~mL}$ QD sample was divided evenly into four centrifuge tubes, $2.5 \mathrm{~mL}$ into each tube. Each tube then received $5 \mathrm{~mL}$ of $\mathrm{EtOH}$, was placed into the centrifuge, and spun at $4000 \mathrm{RPM}$ for 10 minutes.

After centrifugation the supernatant was removed from each tube, working from the top of the liquid down. Another $5 \mathrm{~mL}$ of EtOH was added to each tube and a second centrifugation at 4000 RPM for 10 minutes was performed. The tubes were then inverted and the supernatant poured out leaving the precipitated QDs in the tip of each tube. All four uncapped tubes were placed horizontally in the fume hood with their open ends pointed toward the fume hood door for maximum exposure to air flow. Fourteen hours later the dry tubes were determined to be dry.

After being removed from the fume hood, $1.25 \mathrm{~mL}$ of $\mathrm{CHCl}_{3}$ was added to each tube for a total of $5 \mathrm{~mL}$ of QD suspended in $\mathrm{CHCl}_{3}$. A PL/CHCl 3 solution with a concentration of $10 \mathrm{mg} / \mathrm{mL}$ was made by dissolving 100mg of milk phospholipid powder (Phospholipid Concentration 700, Finterra Ltd, Edgercumbe) into $10 \mathrm{~mL}$ of $\mathrm{CHCl}_{3}$. The amount of $10 \mathrm{mg} / \mathrm{mL} \mathrm{PL} / \mathrm{CHCl}_{3}$ to be made was dependent on the amount of keratinocyte media into which it would be evaporated: $10 \mathrm{~mL}$. 
Using an amount of $\mathrm{PL} / \mathrm{CHCl}_{3}$ equal to the amount of keratinocyte media maintained the desired $10 \mathrm{mg} / \mathrm{mL}$ concentration in the final product. Together this combination created a final ratio of $1 \mathrm{~mL}$ of $\mathrm{QD} / \mathrm{CHCl}_{3}$ for every $20 \mathrm{mg}$ of PL. In the past test trials a 1: 2 ratio of $\mathrm{QD} / \mathrm{CHCl}_{3}: \mathrm{PL} / \mathrm{CHCL}_{3}$ $[2.5 \mathrm{mg} / \mathrm{mL}]$ was used resulting in a final ratio of $1 \mathrm{~mL}$ of $\mathrm{QD} / \mathrm{CHCl}_{3}$ for every $5 \mathrm{mg}$ of PL. To maintain this past ratio it would have been necessary to have added $20 \mathrm{~mL}$ of $\mathrm{QD} / \mathrm{CHCl}_{3}$. The $5 \mathrm{~mL}$ of $\mathrm{QD} / \mathrm{CHCl}_{3}$ and $10 \mathrm{~mL}$ of $10 \mathrm{mg} / \mathrm{mL} \mathrm{PL} / \mathrm{CHCl}_{3}$ were combined in a $50 \mathrm{~mL}$ glass beaker. Added to this was $10 \mathrm{~mL}$ of keratinocyte media. A magnetic spin vane, $2.5 \mathrm{~cm}$ in length, was place into the beaker with the mixture. The beaker was then placed at an angle into a silicon oil bath set at $50^{\circ} \mathrm{C}$ and 200 RPM. The beaker was removed twenty two minutes after being placed into the silicon oil bath.

UV-VIS spectroscopy was used to determine the influence of the encapsulation process on the center max wavelength (CMW), FWHM, and intensity of each QD sample used in the encapsulation. This progression of shifts in the characterization parameters provided a quantitative method for analyzing and predicting final wavelength and intensity measurements. Starting with the CMW, FWHM, and intensity of the CdSe core sample, and continuing to record the same characterization parameters of the CdSe/ZnS QD sample in ODE, the CdSe/ZnS QD sample in $\mathrm{CHCl}_{3}$, the QD:PL: $\mathrm{CHCl}_{3}$, and the final encapsulated QD:PL construct, provided a linear progression of the shifts in the characterization parameters as a direct consequence of each step in the encapsulation procedure. Additional UV-VIS spectrometer data, of control samples, were utilized to determine the influence of the components specific to each step on the characterization parameters. These same UV-VIS characterization parameters were applied to samples of $\mathrm{CHCl}_{3}$, PBS, and variety of PL concentrations in $\mathrm{CHCl}_{3}$ and $\mathrm{PBS}$, all of which added an additional dimension of understanding as to the influence of each step on the final CMW, FWHM, and intensity outcomes. 


\subsection{Results}

\subsubsection{QD Precipitation}

Complete precipitation of the QDs out of ODE was determined based on a qualitative assessment of the centrifuge tubes. The conclusions resulting from the examination of the texture, transparency, and viscosity of the centrifuged QDs dictated the success of the precipitation and whether an additional round of centrifugation was needed. After the first centrifugation the QD/ODE/EtOH solution in the tube had the appearance of image A in Figure 3.4; the majority of the QDs toward the bottom of the tube but still not precipitated into a solid pellet at the tip. After removal of the supernatant, the addition of an equal amount of EtOH and vigorous shaking of the capped tube redistributed the QDs throughout the solution as shown in images B and C of Figure 3.3 .
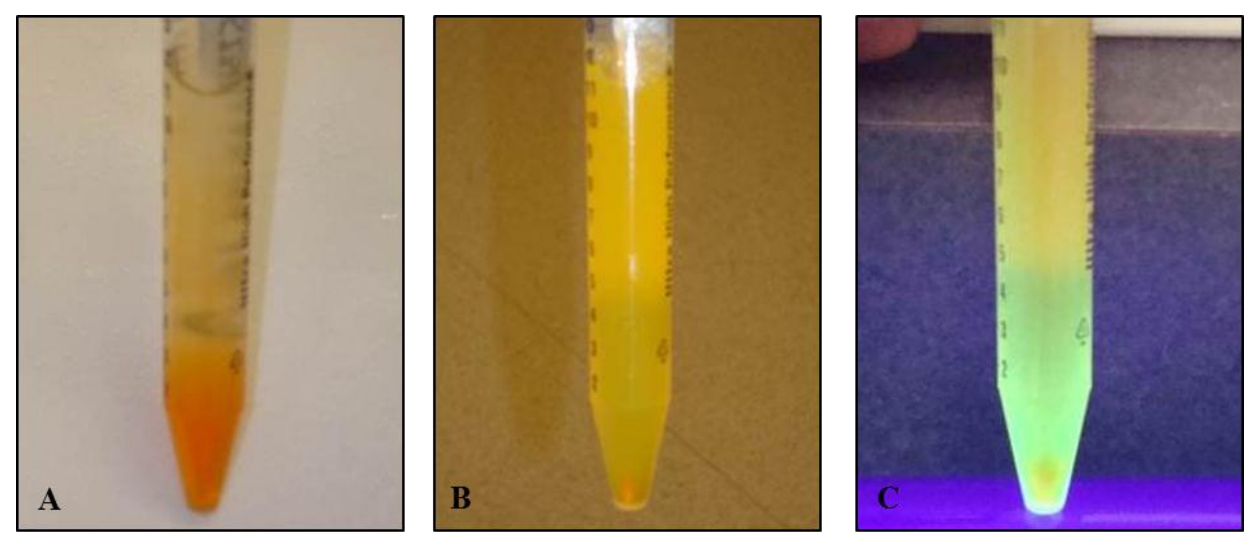

Figure 3.3 QDs suspension in ODE and EtOH after the first series of centrifugation steps. A - after the first centrifugation, before the removal of the supernatant. B - after the addition of EtOH with vigorous shaking in white light. $\mathrm{C}$ - after the addition of $\mathrm{EtOH}$ with vigorous shaking in $\mathrm{UV}$.

\subsubsection{QD Suspension into $\mathrm{CHCl}_{3}$}

In instances where saturation was apparent, QDs were precipitating out of the chloroform as shown in Figure 3.4, an additional amount of chloroform was added in increments of $0.5 \mathrm{~mL}$ until a 
consistent solution of suspended QDs was achieved. It was not always immediately apparent that the chloroform was saturated. On average the final solution was $3 \mathrm{~mL}$ in total.
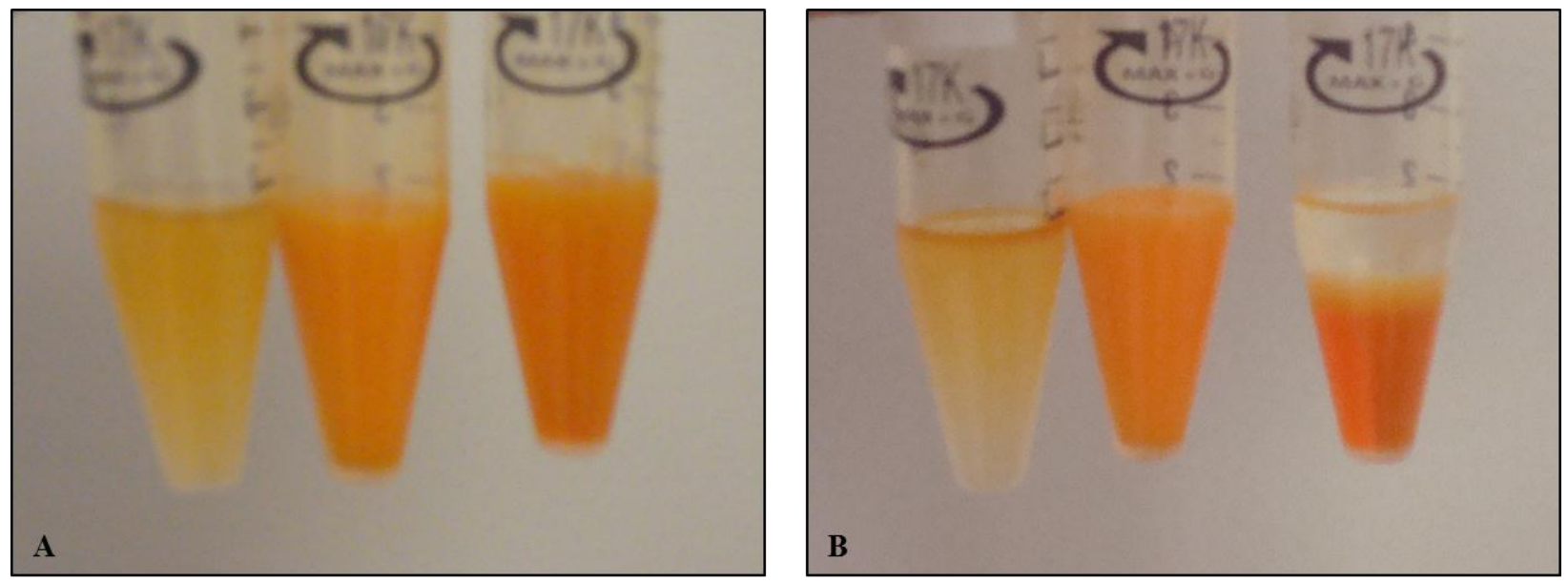

Figure 3.4 A - Re-suspension of QDs in $\mathrm{CHCl}_{3}$. B - Saturation of QDs re-suspended in $\mathrm{CHCl}_{3}$.

\subsubsection{Phospholipid Concentration, Suspension, and Encapsulation}

\subsubsection{Experimental Trials - Round One and Two}

The saturation point of phospholipids in PBS and keratinocyte media was determined to be $10 \mathrm{mg} / \mathrm{mL}$ with the application of heating the mixture to $52^{\circ}$ and vigorous agitation by shaking the capped vial. The saturation point of phospholipids in chloroform was not ultimately determined, but as the chloroform continued to dissolve an amount of $35 \mathrm{mg} / \mathrm{mL}$, more than three times that of the aqueous mediums, further dilutions were not deemed necessary.

Table 3.3 Comparison of QD samples in $\mathrm{CHCl}_{3}$ and QD/PL samples in $\mathrm{CHCl}_{3}$

\begin{tabular}{|l|l|l|l|l|l|}
\hline \multicolumn{1}{|c|}{ Sample } & \multicolumn{1}{|c|}{ Solvent } & $\begin{array}{c}\text { CMW } \\
(\mathrm{nm})\end{array}$ & $\begin{array}{c}\text { Intensity } \\
(\text { counts })\end{array}$ & $\begin{array}{c}\text { FWHM } \\
\text { range }(\mathrm{nm})\end{array}$ & $\begin{array}{c}\text { FWHM } \\
(\mathrm{nm})\end{array}$ \\
\hline 31912QD1.M45m & $\mathrm{CHCl3}$ & 583 & 48000 & $571-598$ & 27 \\
\hline 31912QD1.M45s & $\mathrm{CHCl3}$ & 586 & 47000 & $574-599$ & 25 \\
\hline 31912EN1.M45m & $\mathrm{CHCl3}$ & 575 & 56000 & $556-590$ & 34 \\
\hline 31912EN1.M45s & $\mathrm{CHCl} 3$ & 577 & 45000 & $560-592$ & 32 \\
\hline
\end{tabular}




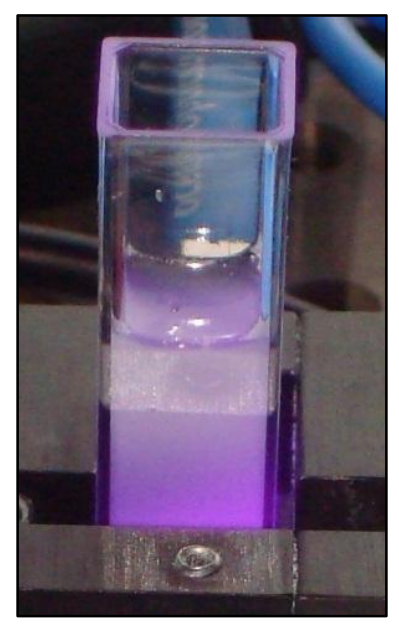

Figure 3.5 A representative image of 0.45 micron filtered 10mg.mL PL/keratinocyte media solution scattering the UV source in the fluorescence detector.

Possible phospholipid related auto-fluorescence issues were investigated with respect to the wavelength at which auto-fluorescence might have occurred. It was concluded that when combined with QDs in $\mathrm{CHCl}_{3}$ the emission of the PL solutions were negligible in range of the QD wavelength of interest. Therefore, although auto-fluorescence was confirmed quantitatively by UVVIS spectroscopy, its influence on the ability to detect the presence of the encapsulated quantum dots was deemed negligible. Fluorescence spectra characteristic of solutions containing phospholipids exhibited dominating peaks at the wavelength of the emission source: $388 \mathrm{~nm}$. Peaks at 500 and $660 \mathrm{~nm}$ were also routinely detected. The size and intensity of each peak was influenced by the solvent in which the PL was dissolved. 


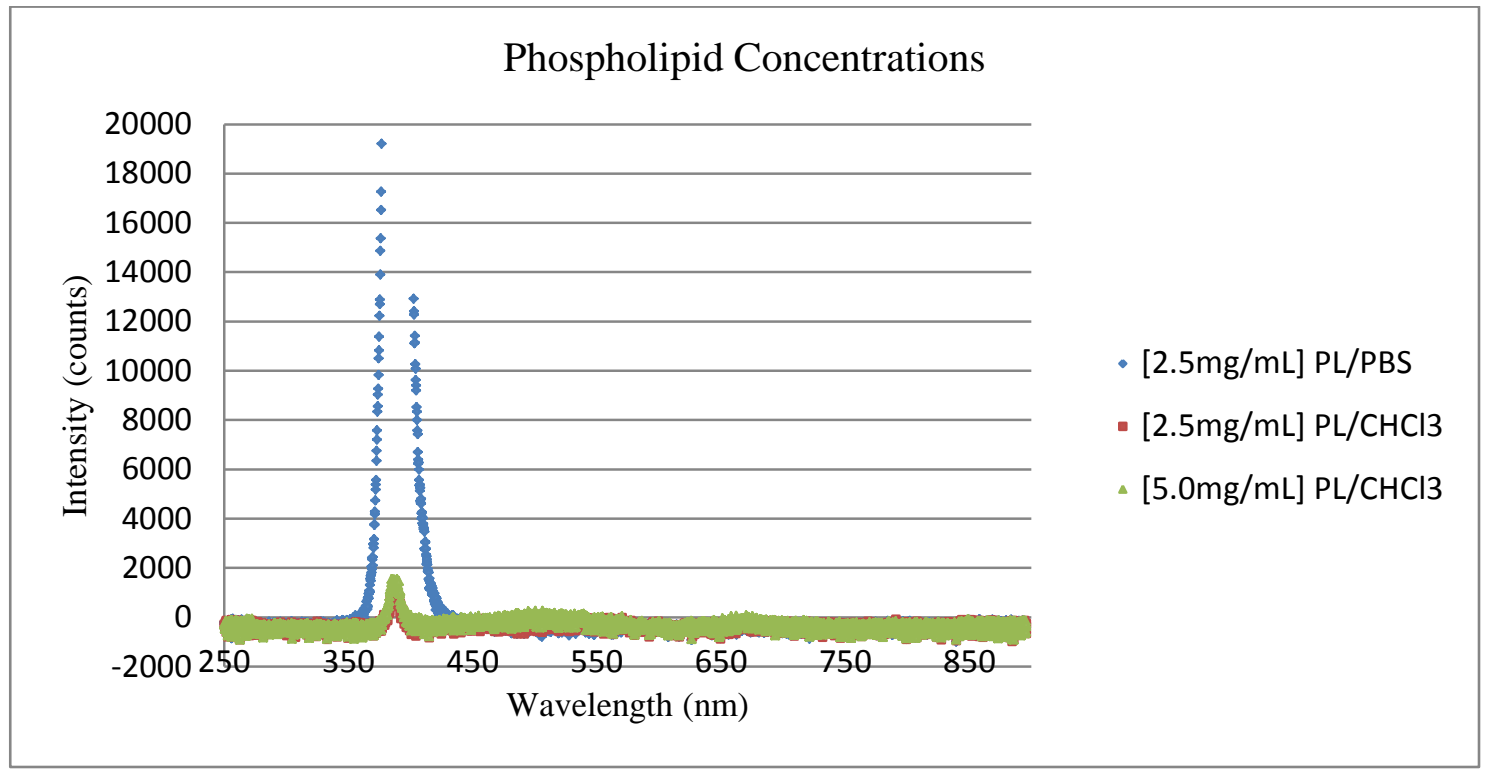

Figure 3.6 Fluorescence spectra of phospholipids at different concentrations, in different medias taken at an integration time of $100 \mathrm{~ms}$; the source peak from scattering occurring at $388 \mathrm{~nm}$.

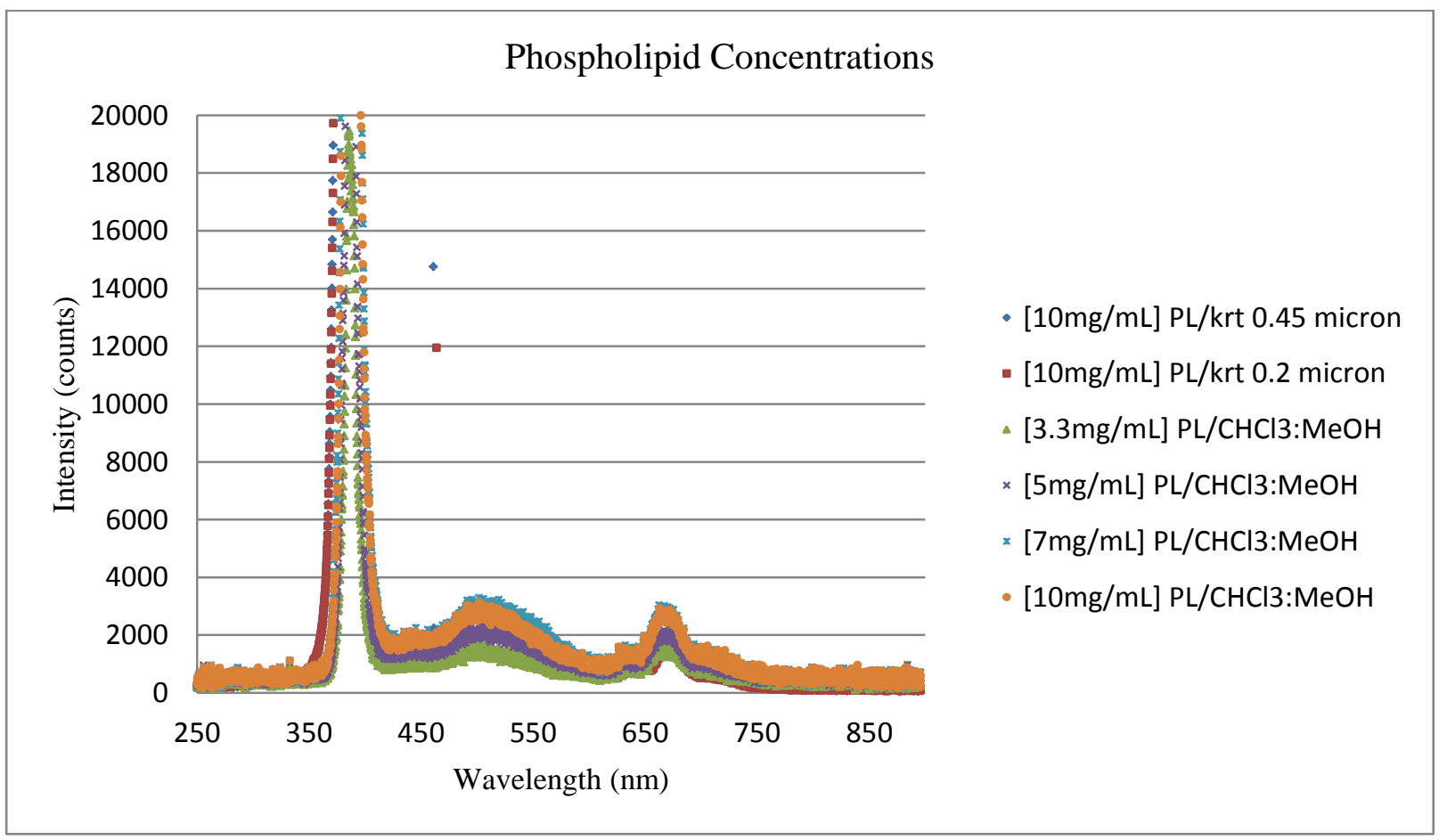

Figure 3.7 Fluorescence spectra of phospholipids at different concentrations, in different medias taken at an integration time of $500 \mathrm{~ms}$; the source peak from scattering occurring at $388 \mathrm{~nm}$. 
For better visualization, the relative size of the fluorescence emission peaks for the solutions with QD constructs and those with only phospholipids the horizontal axis was decreased to only include a range of 450 to $650 \mathrm{~nm}$. It was this range of wavelengths that was pertinent to determining if the auto-fluorescence emission of the phospholipid solutions made a significant contribution to the QD construct emission peaks. It was concluded that the contribution of the phospholipids to the final emission peak of the QD constructs was negligible. Figure 3.9 shows a representative example of the two solutions in direct comparison.

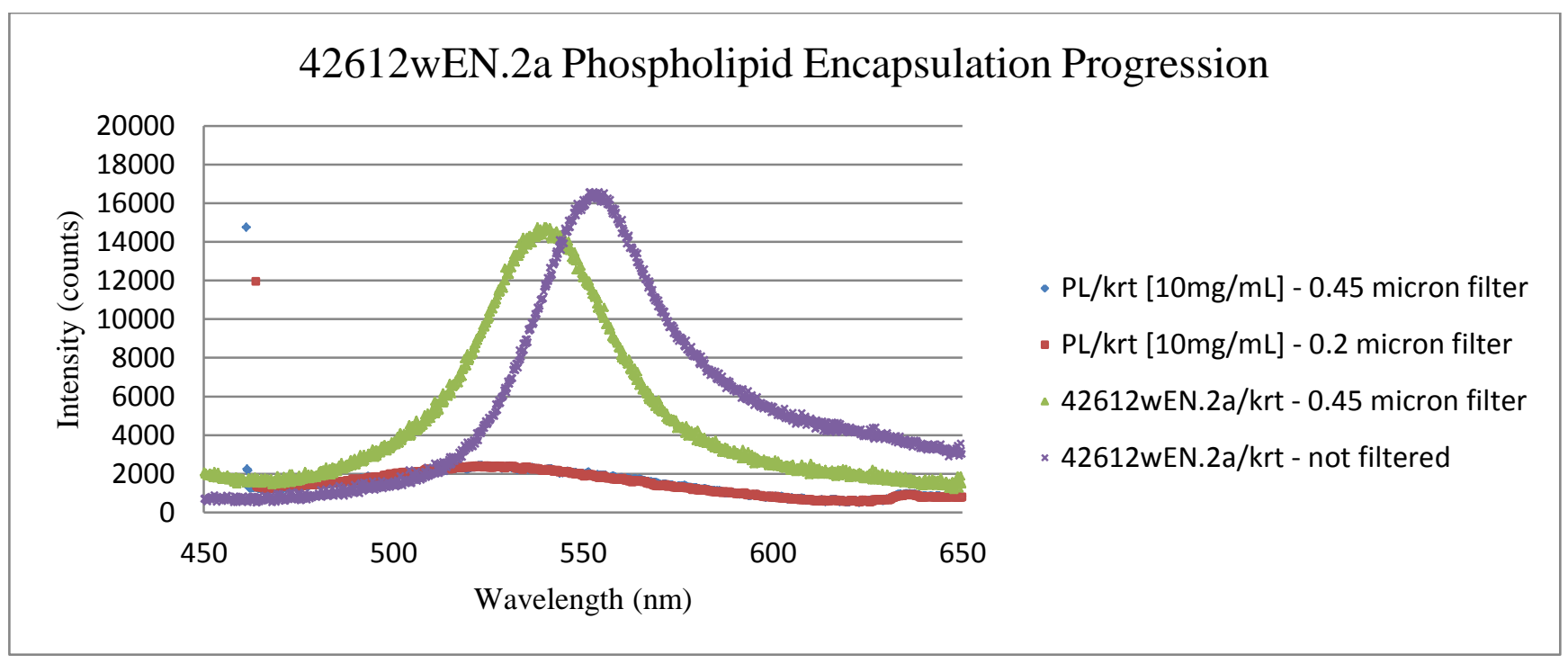

Figure 3.8 Fluorescence spectra of the 0.45 and 0.2 micron filtered $[10 \mathrm{mg} / \mathrm{mL}]$ PL/keratinocyte solutions relative to $\mathrm{QD} / \mathrm{PL} /$ keratinocyte solutions.

An initial evaporation test was performed on solutions of varying concentrations of QD/PL/ $\mathrm{CHCl}_{3}: \mathrm{MeOH}$ and PBS. This initial test was conducted using a minimal application of heat, 10 minutes at $55^{\circ}$, and an extended evaporation time, 2 days, to determine if evaporation alone would produce a final QD construct product using a technique similar to the one described in the Carion et.al. paper. The series of steps was documented and the corresponding images are given in Figures 3.9, 10, and 11. Figure 3.10 shows the solutions after the addition of PBS and 30 minutes of 
vigorous shaking. The final volume of each test solution had decreased at the end of the two day evaporation period, but a significant proportion of the $\mathrm{CHCl}_{3}: \mathrm{MeOH}$ remained and still contained the majority of the QD constructs, with a thin layer of constructs formed in the middle layer as illustrated in Figure 3.11. This result pointed to the need for a method of internal agitation of the solution to be introduced.
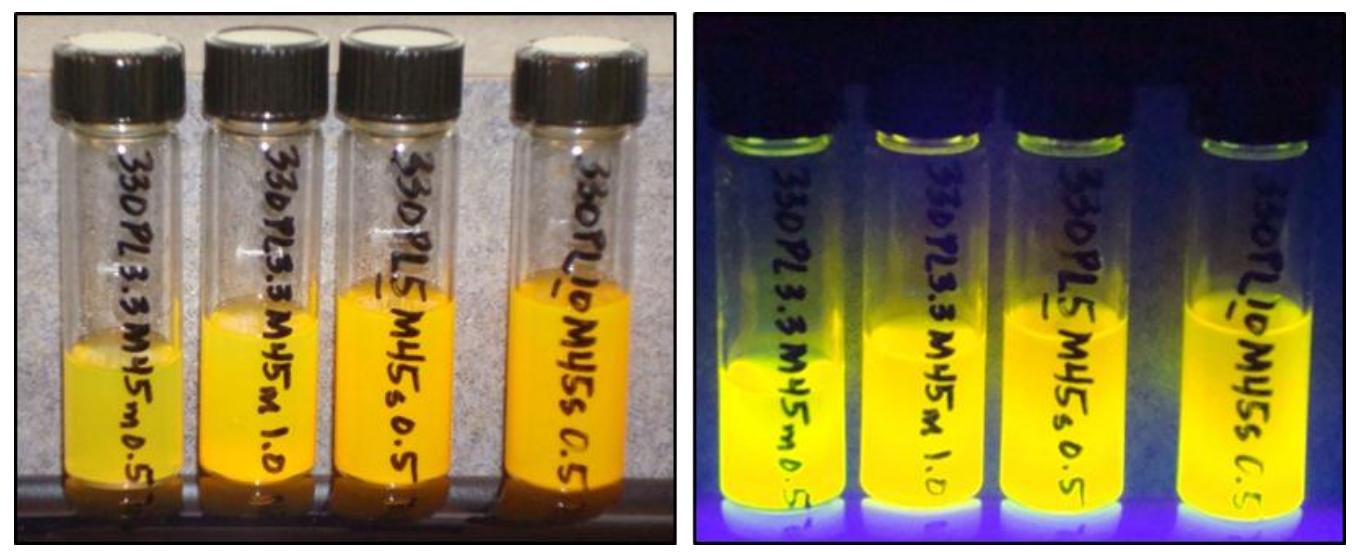

Figure 3.9 Different concentrations of PL $[(3.3,3.3,5.0,10.0) \mathrm{mg} / \mathrm{mL}]$ and QD sample 330QD3.3M45 suspended in $\mathrm{CHCl}_{3} \mathrm{MeOH}$. Left: white light. Right: UV.
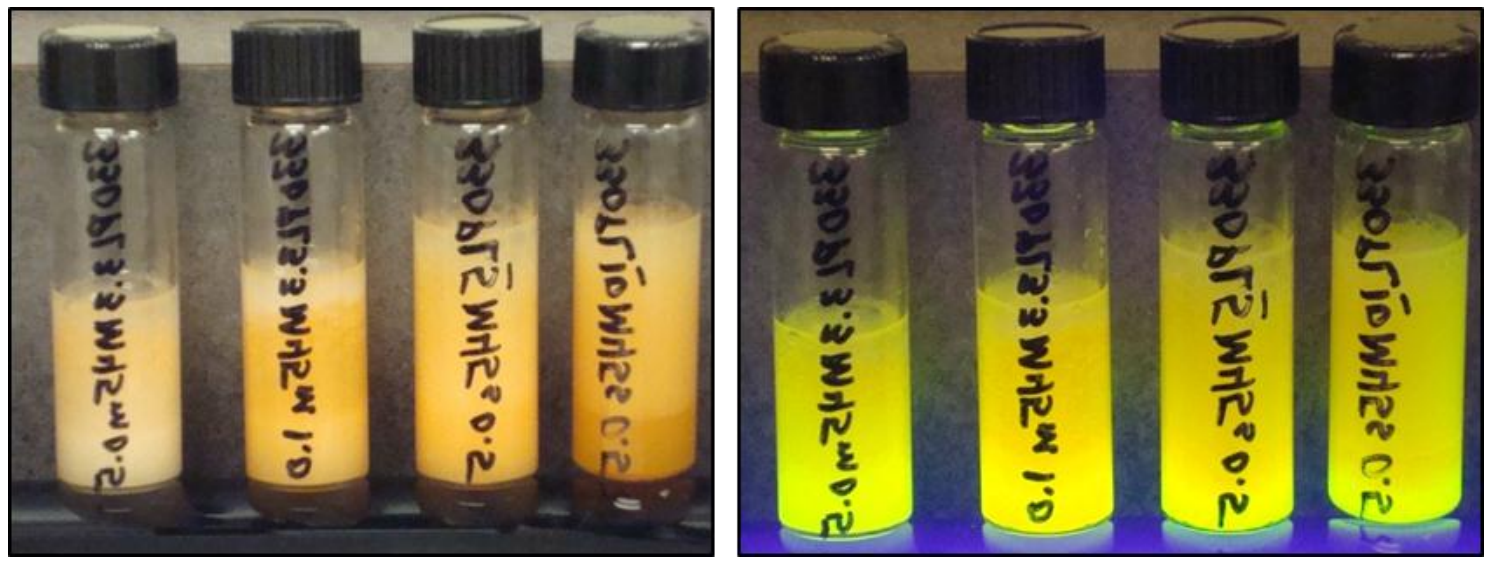

Figure 3.10 Addition of $2 \mathrm{~mL}$ PBS in to each: both in white light and UV. The addition of the PBS was done before evaporation and heating. PBS created an emulsified appearance. Left: white light. Right: UV. 

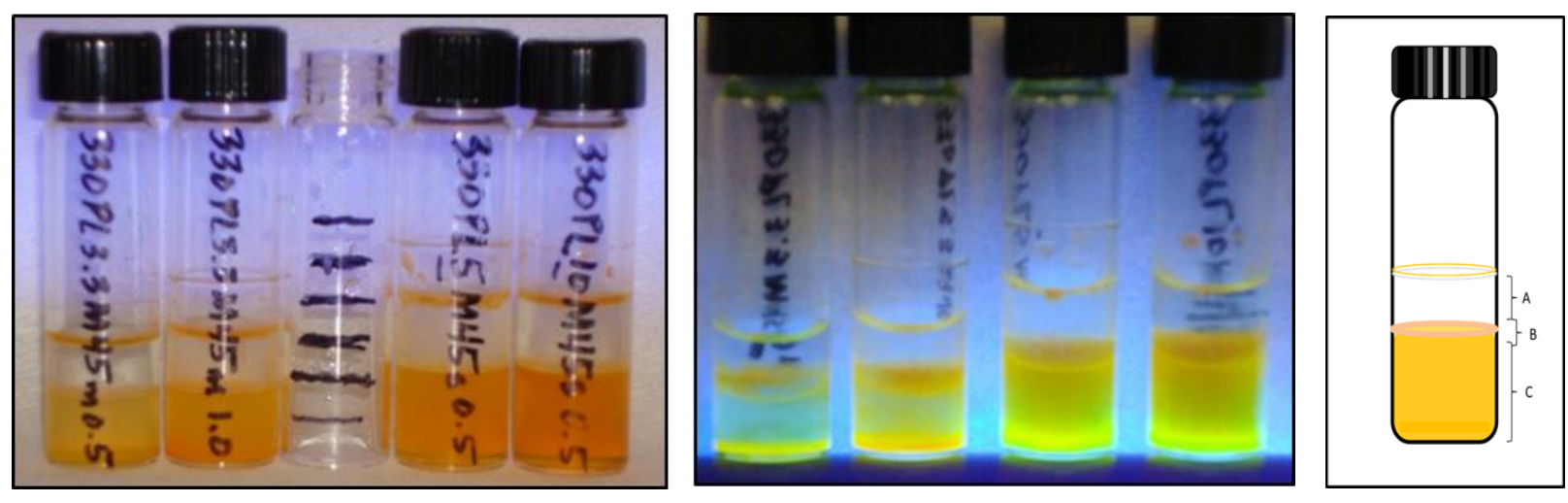

Figure 3.11 After 10min of heating at 55C and 2 days of evaporation in the hood. Left: white light. Middle: UV. Right: Schematic representation of final solution composition. A- PBS. B - QD constructs. C- QD constructs suspended in $\mathrm{CHCl}_{3}: \mathrm{MeOH}$.

Confocal microscopy was used to qualitatively assess how the different concentrations of PL in solvent, and the different ratios of PL to QD in each solution, effected the composition of the QD constructs. Samples of $\mathrm{CHCl}_{3}, \mathrm{PBS}$, and $[2.5 \mathrm{mg} / \mathrm{mL}]$ concentration of PL/PBS were taken as controls for comparison. The smeared quality of the larger aggregates of phospholipids, figures 3.13, and 3.15 are an effect of the increased concentrations of PL within each solvent, as well as decreased ratios of QD to PL resulting in less volume of QDs for every milligram of PL, all resulted in a less uniform and more globular appearance of the QD constructs. Comparison of the control solutions containing [2.5mg/mL] PL/PBS with the test solution consisting of $2.5 \mathrm{~mL}$ of [3.3mg/mL] PL/CHCl $: \mathrm{MeOH}$ and $1.0 \mathrm{~mL}$ of 31912QD1.M45m in $\mathrm{CHCl}_{3}: \mathrm{MeOH}$ shows little difference between the two. The lack of difference introduces an uncertainty as to the degree to which the QDs were successfully encapsulated. Successful encapsulation of QDs within the phospholipid micelles was later confirmed using the fluorescence microscope (images not shown) and the confocal microscope to image the test solutions in the first round of keratinocyte trials. 

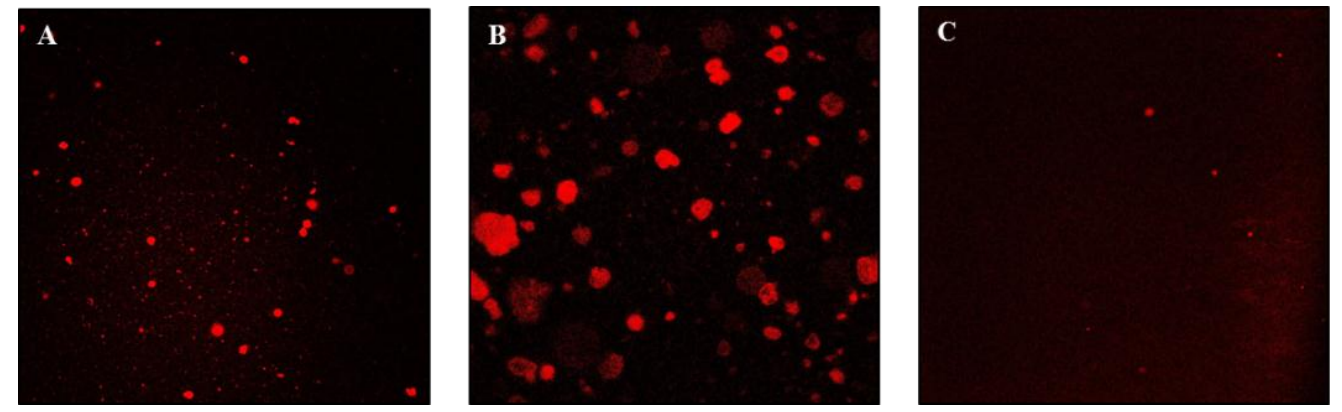

D

Figure 3.12 Controls: A- [2.5mg/mL] PL/PBS at 20X. B - [2.5mg/mL] PL/PBS at 100X. C $[2.5 \mathrm{mg} / \mathrm{mL}] \mathrm{PL} / \mathrm{CHCl}_{3}$ at $20 \mathrm{X}$. D - PBS control
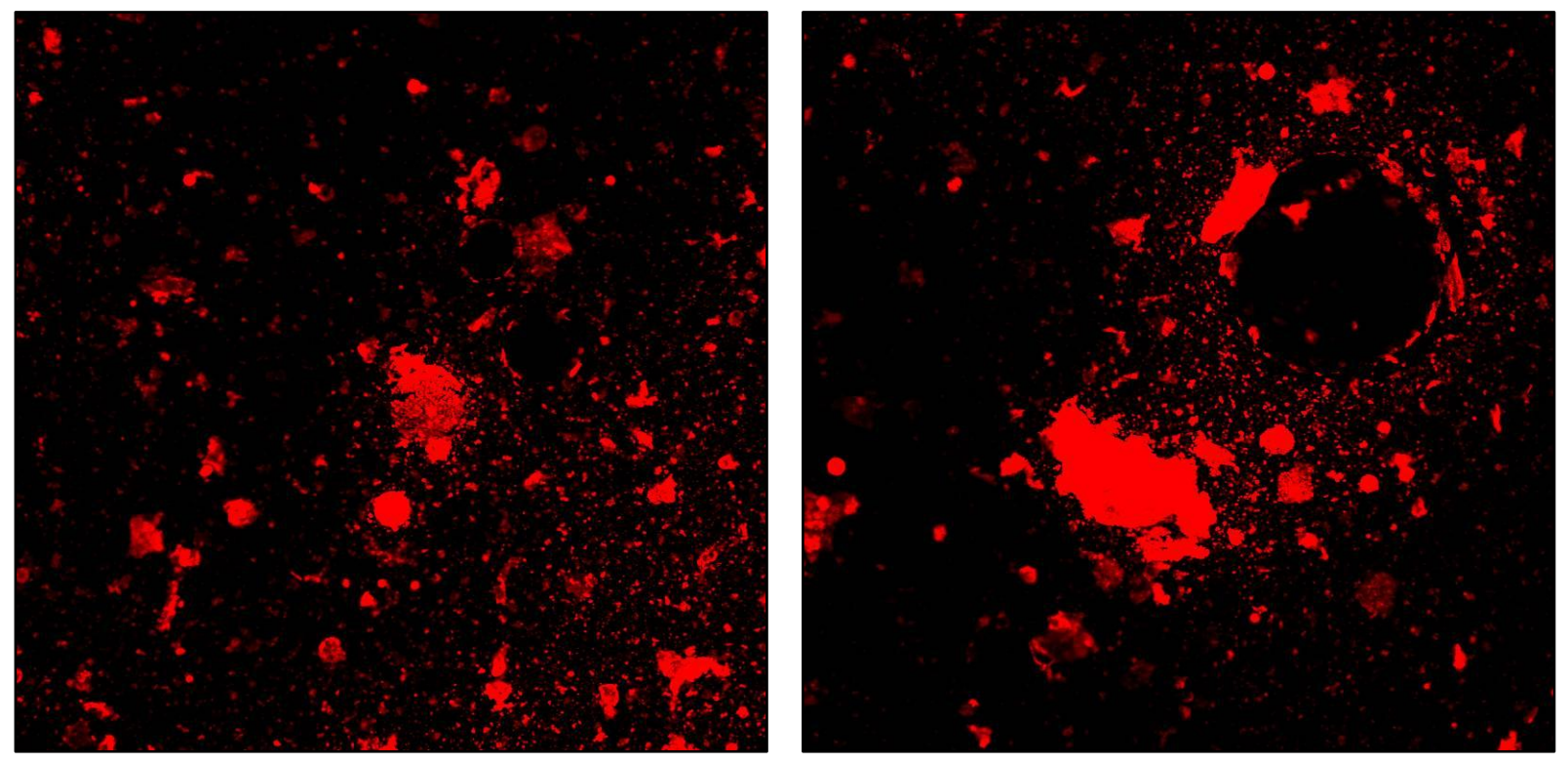

Figure 3.13 A solution of $2.5 \mathrm{~mL}$ of $[3.3 \mathrm{mg} / \mathrm{mL}] \mathrm{PL} / \mathrm{CHCl}_{3}: \mathrm{MeOH}$ and $0.5 \mathrm{~mL}$ of the QD sample 31912QD1.M45m. At precipitation 31912QD1.M45m had been re-suspended in 3.0:1.5

$\mathrm{CHCl}_{3}: \mathrm{MeOH}$ at $20 \mathrm{X}$ 

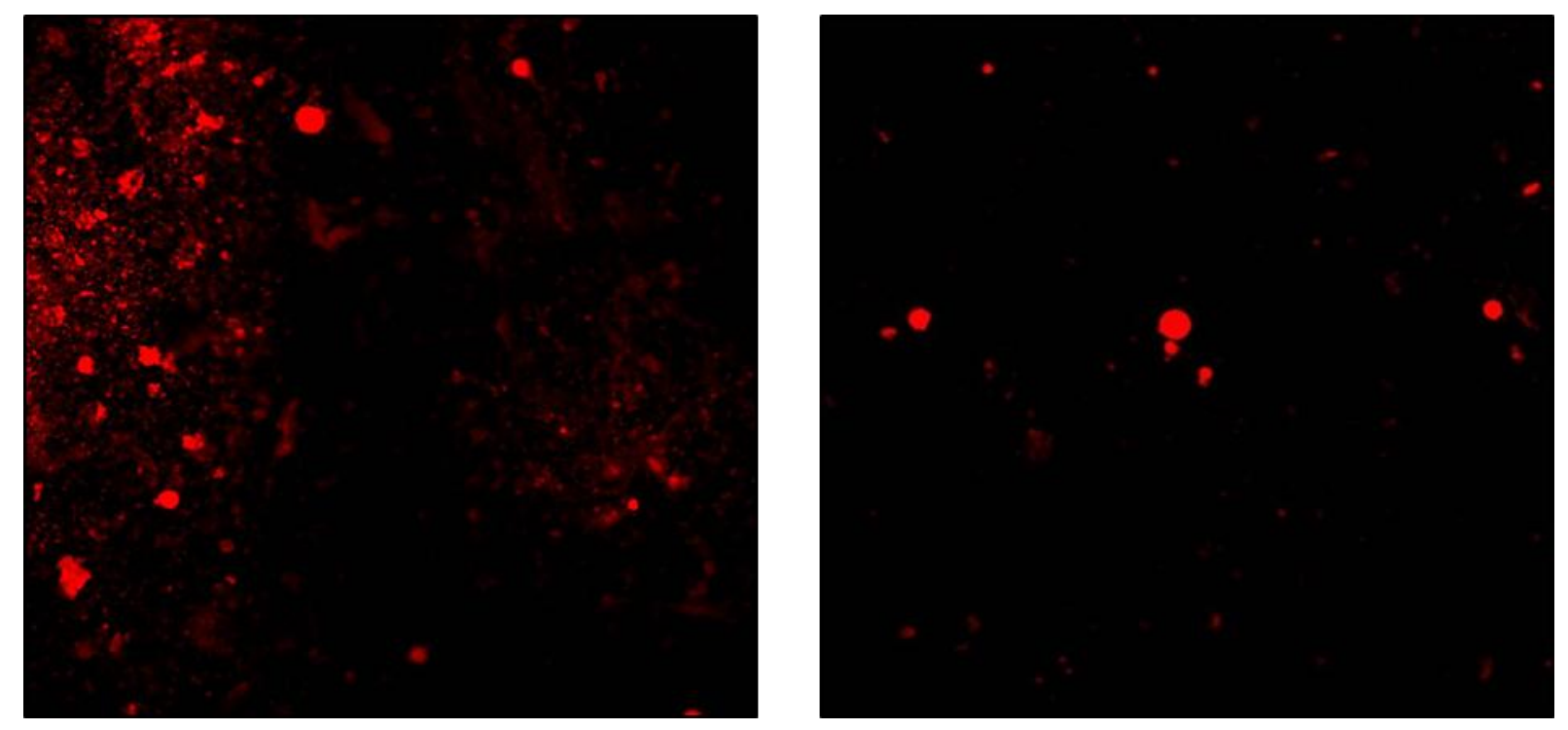

Figure 3.14 A solution of $2.5 \mathrm{~mL}$ of $[3.3 \mathrm{mg} / \mathrm{mL}] \mathrm{PL} / \mathrm{CHCl}_{3}: \mathrm{MeOH}$ and $1.0 \mathrm{~mL}$ of the QD sample 31912QD1.M45m. At precipitation 31912QD1.M45m had been re-suspended in 3.0:1.5 $\mathrm{CHCl}_{3}: \mathrm{MeOH}$ at $20 \mathrm{X}$.
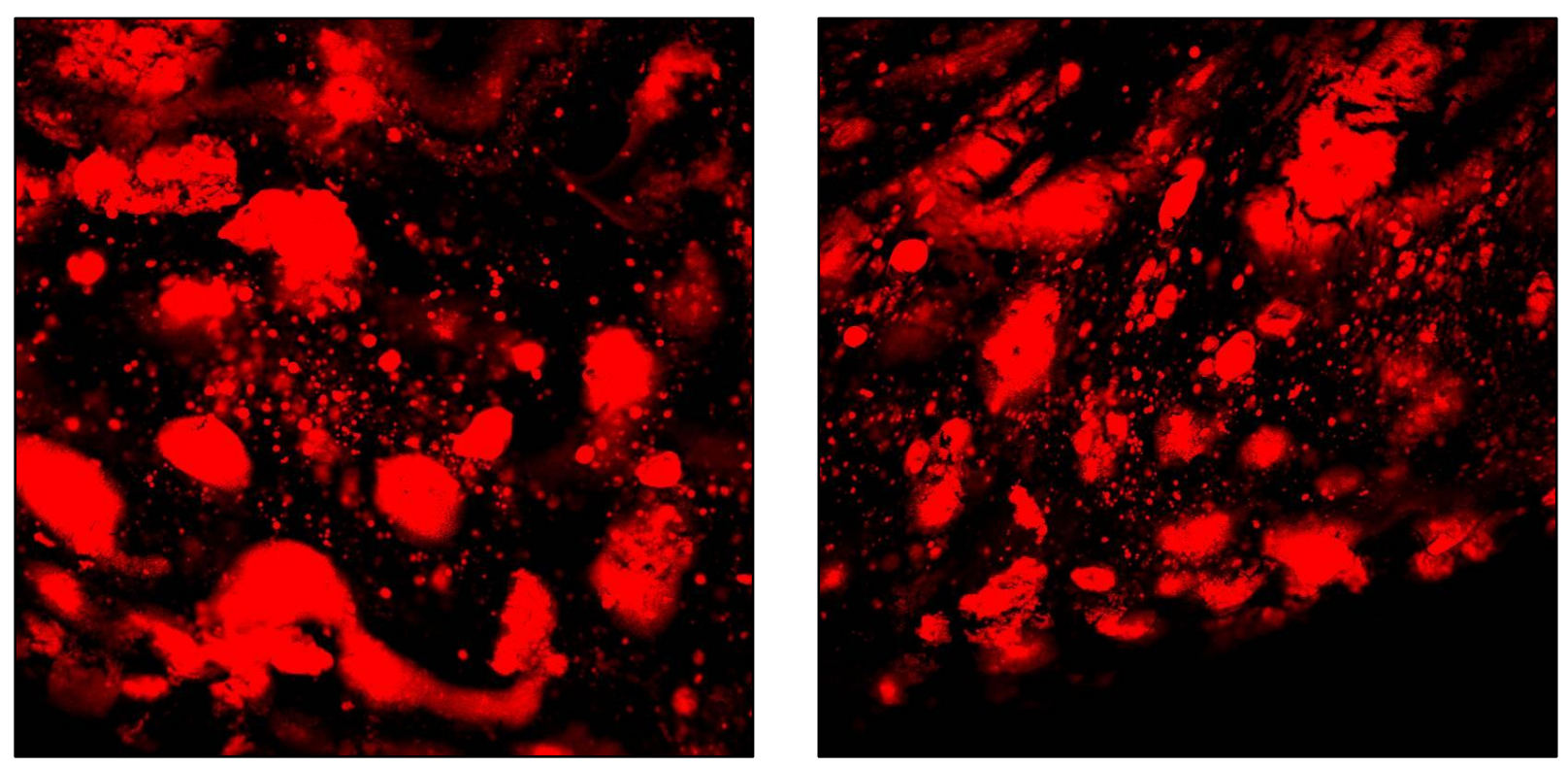

Figure 3.15 A solution of $3.5 \mathrm{~mL}$ of $[10 \mathrm{mg} / \mathrm{mL}] \mathrm{PL} / \mathrm{CHCl}_{3}: \mathrm{MeOH}$ and $0.5 \mathrm{~mL}$ of the QD sample 31912QD1.M45s. At precipitation 31912QD1.M45s had been resuspended in 3.0:1.5 $\mathrm{CHCl}_{3}: \mathrm{MeOH}$ at $20 \mathrm{X}$

In the series of evaporation tests conducted to determine the most successful set of parameters to employ in the final encapsulation and evaporation procedure, it was found that 
application of the nitrogen below the surface of the solution resulted in the creation of dried out strings of QD constructs; defeating the goal of acquiring an even distribution of QDs within the aqueous liquid. Evaporation times in the first round of trials were upwards of 4 hours and it was not discernible whether the remaining liquid was PBS or chloroform. Capping the glass vials with septums and adding a vent needle to increase the pressure within the vial decreased the amount of time that was required to reduce the volume of clear liquid within the vial, but did not answer the question as to what the identity of the remaining liquid. A preliminary test for determining the presence of chloroform was to expose a plastic disposable cuvette to the solution in question for a period of 5 minutes. If the surface of the plastic in contact with the solution degraded, the presence of chloroform was confirmed. Increasing the diameter of the container used during evaporation increased the rate of evaporation of $\mathrm{CHCl}_{3}$ when used in concert with a magnetic spin vane set to a minimum of 200RPM. Applying heat to the solution also increased the rate of evaporation.

A visual representation of the progression of sample D from the first round of encapsulation tests is given in Figure 3.16. The progression starts with the original sample, 32412QD23.15A, suspended in $\mathrm{CHCl}_{3}$ with PL shown in white light and $\mathrm{UV}$, through to its appearance after testing had concluded. The three vials of sample D never individually achieved full evaporation of $\mathrm{CHCl}_{3}$, however, after combining all three and applying the final encapsulation method the $\mathrm{CHCl}_{3}$ was fully evaporated and the QD constructs suspended into PBS. 


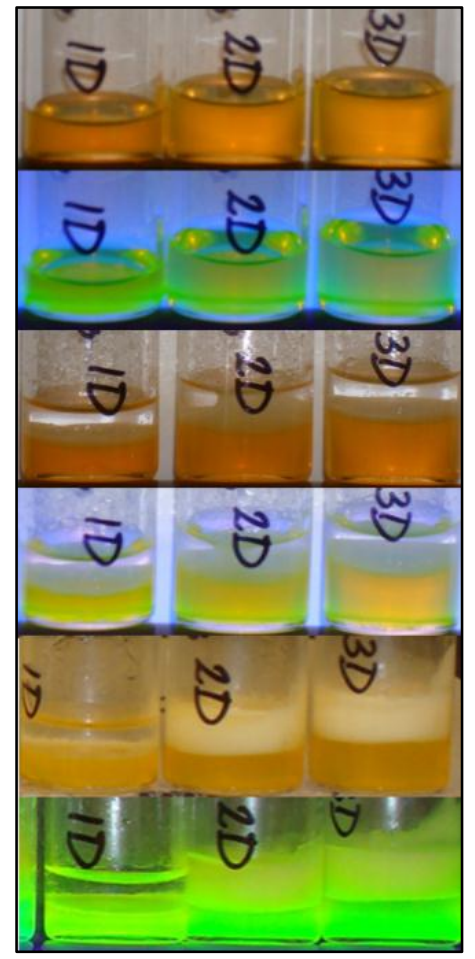

Figure 3.16 Encapsulation progression of set D. From top to bottom: 32412QD23.15A suspended in $\mathrm{CHCl}_{3}$ with PL in white light, in UV light, addition of $2 \mathrm{~mL}$ PBS in white light, in UV light, final state post-evaporation in white light, and in UV light.

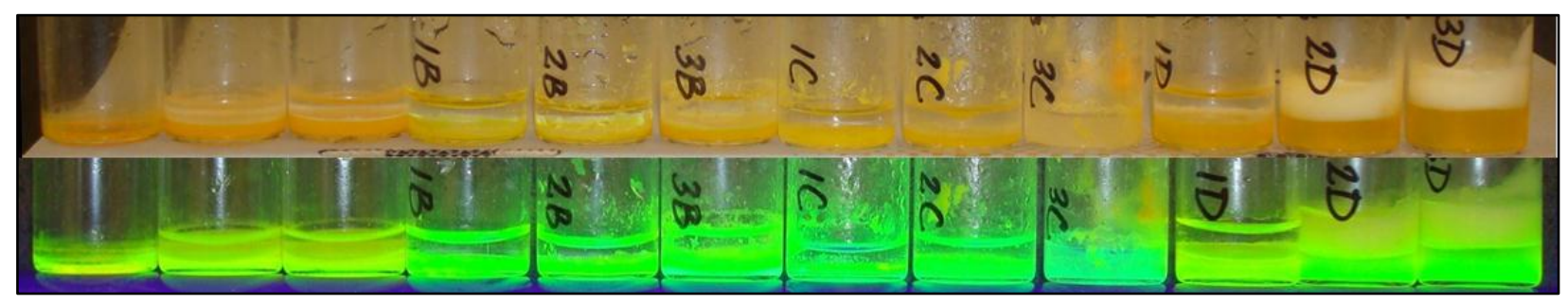

Figure 3.17 Encapsulation progression of sets A, B, C, and D from left to right. Top: white light. Bottom: UV light.

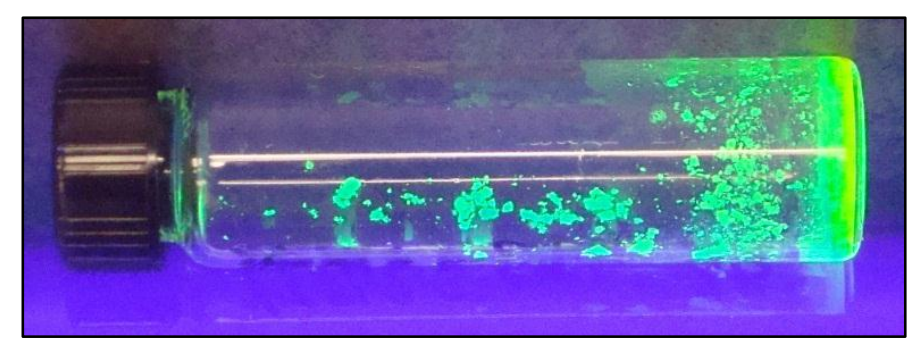

Figure 3.18 Encapsulation trial 2B. Starting solution of $2 \mathrm{~mL} 2.5 \mathrm{mg} / \mathrm{mL}$ of $\mathrm{PL} / \mathrm{CHCl}{ }_{3}: 1 \mathrm{~mL}$ 324QD123.60a in $\mathrm{CHCl}_{3}: 2 \mathrm{~mL} \mathrm{PBS}$ in an open vial heated at $55^{\circ} \mathrm{C}$. 


\subsubsection{Final Encapsulation Method}

The solution of $5 \mathrm{~mL}$ of $\mathrm{QD} / \mathrm{CHCl}_{3}$ and $10 \mathrm{~mL}$ of $10 \mathrm{mg} / \mathrm{mL} \mathrm{PL} / \mathrm{CHCl}_{3}$ appeared consistent throughout. The mixture resulting from the addition of the keratinocyte media to the $\mathrm{QD} / \mathrm{PL} / \mathrm{CHCl}_{3}$ formed two separate layers as illustrated in Figure 3.19. The bottom layer of the mixture is the higher density $\mathrm{QD} / \mathrm{PL} / \mathrm{CHCl}_{3}$ solution and the top layer is the lower density aqueous keratinocyte media. As the image on the left indicates, there is complete separation of the two layers. Over time the viscosity of the solution thickened until a substance the consistency of yogurt was formed. A series of images depicting the change in the mixtures appearance and viscosity is given in Figure 3.20. Twenty two minutes after being placed into the silicon oil bath all remaining clear liquid had been absorbed or evaporated.
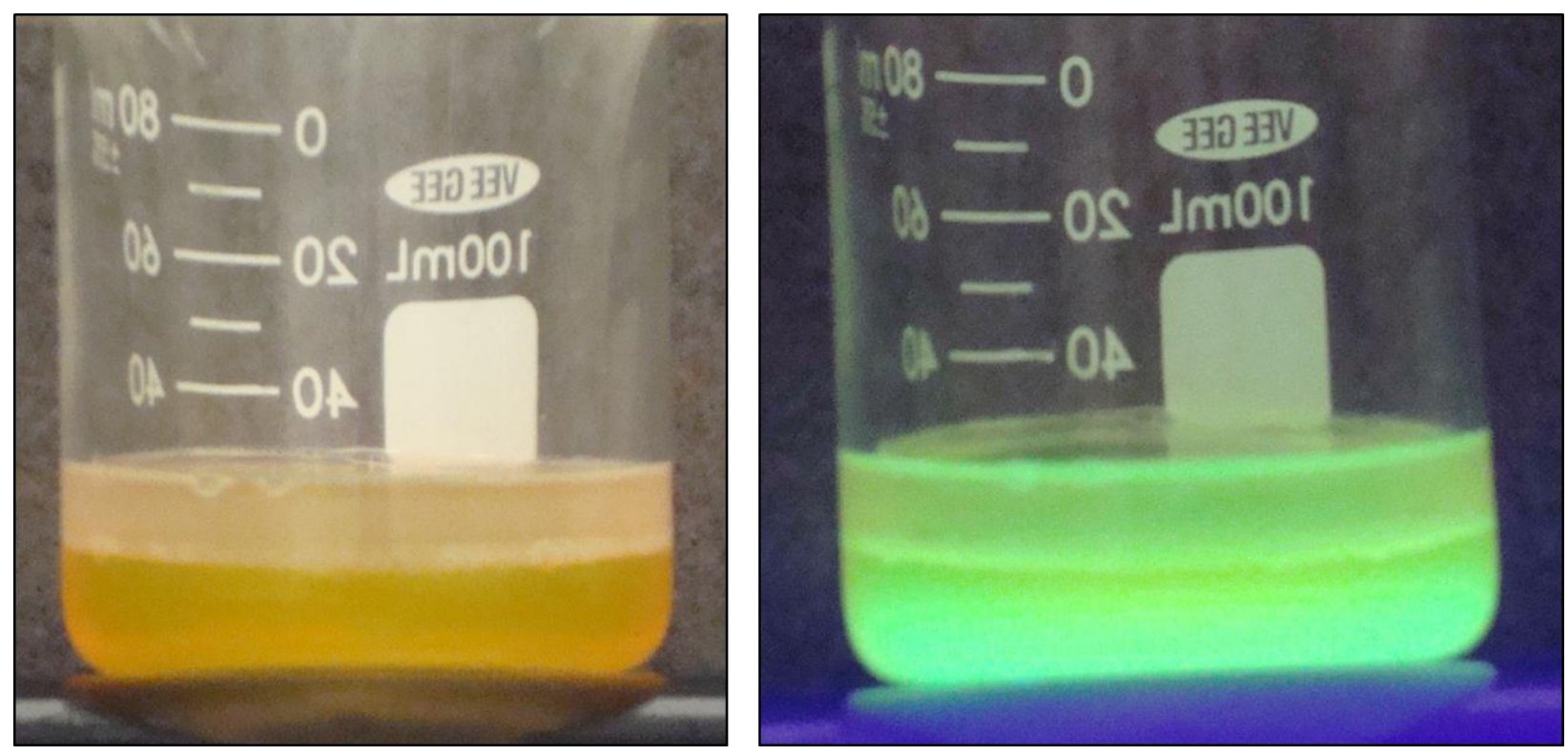

Figure 3.19 Pre-evaporation combination of $5 \mathrm{~mL}$ of $\mathrm{QD} / \mathrm{CHCl}_{3}: 10 \mathrm{~mL}$ of $10 \mathrm{mg} / \mathrm{mL} \mathrm{PL} / \mathrm{CHCl}_{3}$ : $10 \mathrm{~mL}$ keratinocyte media in white light (left) and UV light (right) 

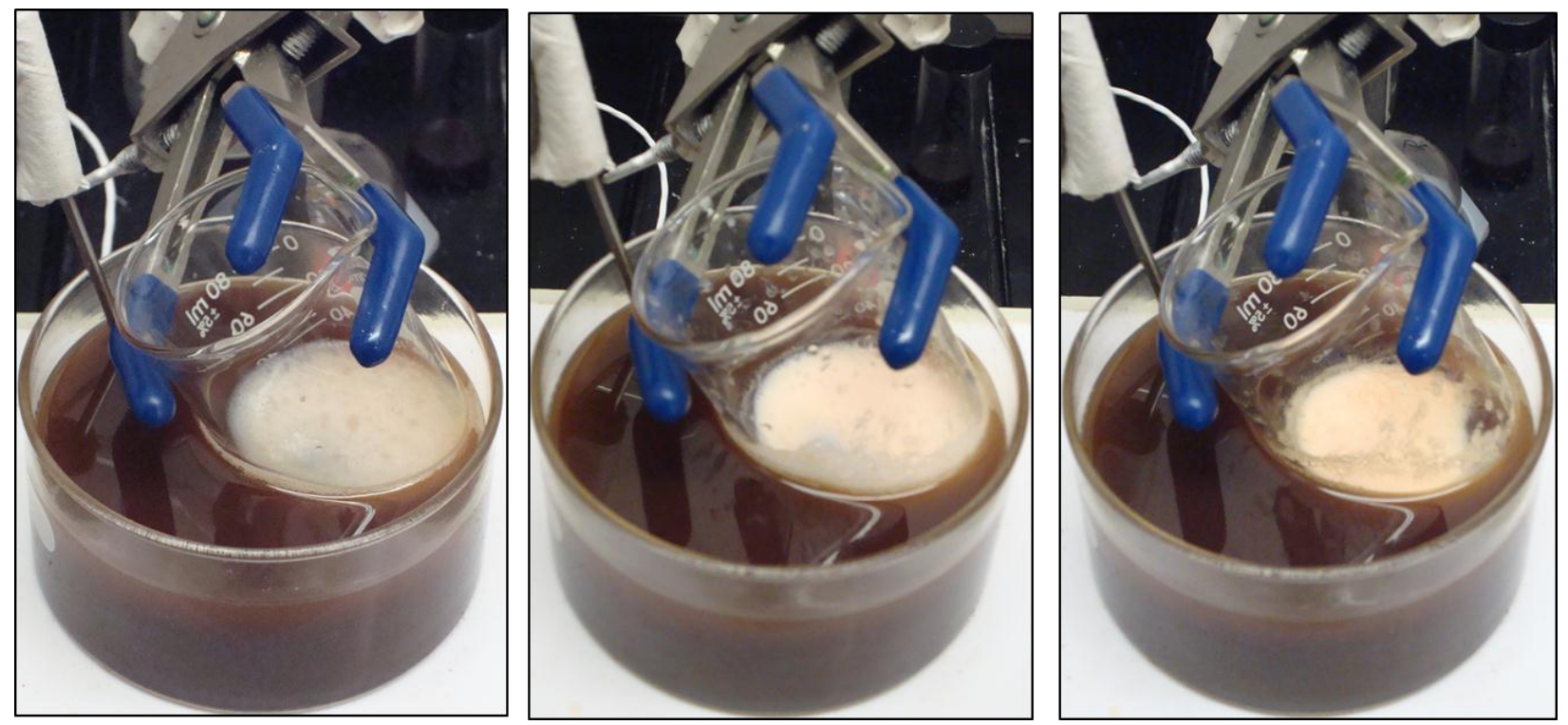

Figure 3.20 Mid-evaporation of $5 \mathrm{~mL}$ of $\mathrm{QD} / \mathrm{CHCl}_{3}: 10 \mathrm{~mL}$ of $10 \mathrm{mg} / \mathrm{mL} \mathrm{PL} / \mathrm{CHCl}_{3}: 10 \mathrm{~mL}$ keratinocyte media at 5, 10, and 15 minutes from left to right.

\subsection{Discussion}

\subsubsection{QD Precipitation}

Typically only two rounds of precipitation steps were necessary to achieve a solid precipitate in the tip of the centrifuge tube; on occasion three rounds. It was discovered through the many precipitations that if more than three rounds were required, the results of products further down the line were often less than desirable. For this reason any attempt at precipitation that did not yield a solid precipitate in the tip of the tube by the third round was discarded unless the QD sample was of particular importance.

The previous method of precipitation involved placing the entire QD sample in one tube and using a second tube of water, equal in weight to the QD sample, to act as a balance during centrifugation, was found to be tedious and overly time consuming. As a result of the $12 \mathrm{~mL}$ maximum capacity of each centrifuge tube, handling QD samples of more than 5mL, which would have required $10 \mathrm{~mL}$ of $\mathrm{EtOH}$, resulted in 4, 5, and 6 rounds of the precipitation steps to achieve a 
solid precipitate in the tip of the tube. As an antidote to this problem, the multiple centrifuge tube method was used. Not only did this decrease the time necessary to precipitate the QDs, but multiple samples, up to six, could be simultaneously centrifuged as long as care was taken to ensure that all tubes contained equal volumes of mixtures.

With regard to the formation of black precipitates in the tip of the centrifuge tubes, it was concluded that the black particulate that sometimes formed was selenium. It was determined to be selenium because of the resemblance of the precipitate to the starting material used in the synthesis; selenium powder was the only component that was also black. Precipitation of selenium had occurred for one of two reasons: the initial precipitated sample had been that of unshelled and therefore unstable CdSe cores, or the initial precipitate was of a shelled sample that had not been consistently and effectively shelled leading to the same outcome. In carefully and consistently synthesized CdSe/ZnS QDs the black precipitate was a rare occurrence.

\subsubsection{Phospholipid Concentration, Suspension, and Encapsulation}

\subsubsection{Experimental Trials - Round One and Two}

Fluorescence spectra of PL in a variety of solvents showed both an element of autofluorescence in the 500 and $660 \mathrm{~nm}$ peaks as well as scattering of the source at $388 \mathrm{~nm}$. The peak from the source was so large as to be larger than what the spectrum would accommodate, however, this peak was only significant when the solvent was either PBS or keratinocyte media or contained $\mathrm{MeOH}$. The solutions consisting of only $\mathrm{PL}$ and $\mathrm{CHCl}_{3}$, no matter the concentration of PL, had a relatively insignificant peak at $388 \mathrm{~nm}$.

Confocal microscope images obtained using the Qdot565 filter were used as a qualitative assessment of the encapsulation process for different concentrations of PL in solvent and different ratios of PL to QD. The images of the control solutions support the conclusion that the PLs have a 
greater affinity for aggregating together as micelles and liposomes when suspended in an aqueous solution. The controls also eliminate the possibility that the solvents are introducing any particulates. The confocal images in Figures 3.13, 14, and 15 support the conclusion that solutions with smaller concentrations of PL and a more equal ratio of PL to QD produce more consistently sized and spherical QD constructs. The conclusions made as a result of the initial PL concentration tests would have been used in the final method if not for the desire to achieve a $10 \mathrm{mg} / \mathrm{mL}$ concentration of QD constructs in keratinocyte solution for mimicking the media parameters described in the Russell et al. paper.

The greatest difficulties encountered in the encapsulation process were consistent and complete evaporation of the chloroform in a timely manner, and the consistency of the final QD construct aqueous solution. Without exposure to the air it was not possible to remove the chloroform form the mixture via evaporation. This particular difficulty, the evaporation of the $\mathrm{CHCL}_{3}$, was the catalyst for the development of so many evaporation strategies. The densities of each element had a significant effect on the development and success of the procedure. The solubility of chloroform in water is $\mathrm{g} / 100 \mathrm{~mL}$ at $20^{\circ} \mathrm{C}: 0.8$ making chloroform essentially immiscible in water (79). The density of chloroform relative to water is $1.48: 1.0\left(\mathrm{~kg} / \mathrm{m}^{3}\right)$ at $20^{\circ} \mathrm{C}$. When the two solvents were mixed, this attribute caused chloroform to sink to the bottom and for the water, PBS, or keratinocyte media to float to the top; water and PBS have equivalent solubility and density characteristics relative to chloroform (79). The immiscibility of chloroform in water based solvents, coupled with chloroforms' density prevented the QD:PL constructs from achieving suspension in the upper layer.

The three main literature references used in the initial phases of developing the encapsulation method laid out in this thesis were Fan et.al., Carion et. al., and Dubertret et. al. (1113). The Fan et. al. paper proposed encapsulation by the introduction of the $\mathrm{QD} / \mathrm{CHCl}_{3}$ suspension 
to an aqueous lipid solution with the application of vigourous stirring to create a micro-emulsion that was then to be heated between 40 and $80^{\circ} \mathrm{C}$ for approximately ten minutes to evaporate off the chloroform and transfer the QDs into the aqueous solution. The Dubertret et. al. paper used "a mixture of $n$-poly(ethylene glycol) phosphatidylethanolamine (PEG-PE) and phosphatidylcholine (PC) and did not explicitly state there method for encapsulation. The only indication for what process was used to achieve encapsulation was a diagram indicating that after being combined the QD/PL solution was evaporated, heated, and then water was added. This was less than helpful and all of my tests that applied evaporation and heating before adding the aqueous medium did not yield successful results. The Carion et. al. paper was thorough and explicitly detailed each step used in their process. It was by far the best source available in the literature. The procedure outlined in the Carion paper was quoted as taking 6 hours and utilized equipment and ingredients not available at the time of the work for this thesis. It would, however, be a worthwhile method to investigate for the future.

\subsubsection{Final Encapsulation Method}

The deviation of the final encapsulation method, with respect to the ratio of milliliters of $\mathrm{QD} / \mathrm{CHCl}_{3}$ to milligrams of $\mathrm{PL}$, from the test trials was a result of trying to duplicate the concentration of PL in keratinocyte media that was used in the Russell et al. paper. Ultimately, the goal was to introduce the QD constructs into EpiDerm skin substitutes. For this reason using a solution that mimicked the original $1 \% \mathrm{w} / \mathrm{v}$ phospholipid/keratinocyte media, like that which had been used in the Russell experiments, would eliminate the one variable in the effect of the QD constructs on the skin substitutes. It was, however, noticed that compared to the original tests, which had optimum results using a ratio of $1 \mathrm{~mL}$ of $\mathrm{QD} / \mathrm{CHCl}_{3}$ for every $5 \mathrm{mg}$ of $\mathrm{PL}$, and $2 \mathrm{~mL}$ aqueous media for every $1 \mathrm{~mL}$ of $\mathrm{QD} / \mathrm{PL} / \mathrm{CHCl}_{3}$, the final method employed tended to create a more coagulated product, which $66 \%$ of the time resulted in difficulty during filtering. The 
difficulty in filtering stemmed from an increase in the viscosity and uneven distribution of the QD constructs throughout the aqueous media. Overall, the final encapsulation method is a process that from start to finish can be accomplished in approximately 3.5 hours. 


\section{Chapter 4 - Introducing Quantum Dot Constructs into a Biologic Medium}

\subsection{Introduction}

Upon achieving successful encapsulation of the CdSe/ZnS QDs within phospholipid micelles, and evaporating them into an aqueous media, the next step was to introduce the QD construct solution into a biologic medium. The biologic mediums chosen for this purpose was keratinocyte cell cultures. Introduction of the QD constructs into keratinocyte cell cultures was performed to assess two parameters of QD-cell interaction: QD-cell integration and cell health post-introduction of QD construct solutions. The success of QD-cell integration was qualitatively assessed using the ratio of the number of cells with QD constructs attached to their cellular membranes relative to the number of cells without any QD constructs attached to their membranes. In addition to attachment of QD constructs to the exterior of cell membranes, the degree of integration into the keratinocyte cells was also assessed. The effect of the QD constructs on cell health, post-introduction of the test solutions, was measured by a comparison of cell viability, proliferation, and morphology from before and after the introduction of the QD constructs test solutions.

Evaluation of the effects of QD-construct solutions on the health of the keratinocyte cells was done using imaging and histology techniques. An optical white light Olympus CKX41 microscope was employed for the comparison of changes in cell morphology and culture appearance and used as a qualitative assessment of the effects of QD construct solutions on cell health. Calcein and Ethidium homodimer-1 (EthD-1) live dead (L/D) stains were used in concert with confocal and DIC imaging to determine the percentage of live to dead cells within each fourwell chamber. QD construct adherence to, and integration within, keratinocyte cells was done by qualitative inspection of confocal and DIC images. Histochoice fixative and Hoechst stain $(\mathrm{H} / \mathrm{H})$ 
were used to identify the nucleus of each cell, thereby making it possible to accurately differentiate QD constructs exterior to the cell membrane surface relative to QD constructs interior of the cell membrane surface. Application of the Hoechst stain also allowed for distinguishing the keratinocyte cells relative to any unwanted contamination sources, such as spores, molds, or fungus that may have been present and which the QD constructs may have also interacted with.

\subsection{Methods}

\subsubsection{QD Construct Introduction into Keratinocytes Cell Cultures}

The cells to which the QD construct test solutions were applied were Primary Epidermal Keratinocytes; normal (non-diseased), human, from neonatal foreskin; supplied by ATCC, product number PCS-200-010. The media for the keratinocytes, also supplied by ATCC, was a base solution of dermal cell basal medium with keratinocyte growth kit. The keratinocyte growth kit, product number PCS-200-010, included $2 \mathrm{ml}$ of bovine pituitary extract, $0.5 \mathrm{ml} \mathrm{rh}$ TGF-alpha, 15

$\mathrm{ml}$ L-glutamine, $0.5 \mathrm{ml}$ hydrocortisone hemisuccinate, $0.5 \mathrm{ml} \mathrm{rh}$ insulin, $0.5 \mathrm{ml}$ epinephrine and 0.5 $\mathrm{ml}$ apo-transferin. The cells were thawed from cryopreservation and passed into T75 flasks. The cells were incubated at $37^{\circ} \mathrm{C}, 5 \% \mathrm{CO}_{2}$, and $95 \%$ humidity in the primary incubator, and given fresh media every 36 -48 hours (80). All cell culture handling and maintenance, pre-introduction of test solutions, was conducted by Carl T. Dargitz.

A maximum time point of 24 hours was chosen for the keratinocyte QD construct experiments as a consequence of the realities associated the maintenance of an aseptic culture environment for the duration of the keratinocyte related trials. The uncertainty for maintaining an aseptic environment stemmed from three different factors: the design of the four-well flasks, working outside of the laminar flow hood, and using the secondary not aseptically maintained dry incubator for incubation post-introduction of the test solutions. Unlike the T75 culture flasks that 
the cells were initially grown in, the four-well slides are not sealable with a filter-cap. This factor increases the likelihood that any environmental contaminants that may be present will likely invade the culture and affect the viability of the cells. To combat the constraints of working in a nonaseptic environment, handling of the keratinocyte cultures was done wearing nitrile gloves sprayed down with 70\% isopropyl alcohol (IPA). All surfaces, counter-tops, the incubator shelves and door, and the optical microscope stage platform, which the four-well slides containing the keratinocyte cultures came in contact with, were also sprayed down with 70\% IPA and air dried before any contact was initiated. This in no way ensured that contamination of the cultures would be prevented. However, this precaution, along with the short time point of 24 hour postintroduction of testing of solutions, prevented contamination from reaching levels that would detrimentally affect the experimental parameters of interest.

\subsubsection{QD Construct Control and Test Solutions - RI}

In the first round (R1) of test solutions an extensive set of control solutions were employed in an effort to identify the influence of non-QD construct specific variables on the viability of the keratinocyte cells. Each step involved in the process of developing the final QD construct test solutions contributed variables, which if untested, would introduce sources of unknown influence and variability on the cell health and cell-QD construct interaction of the final product. The test solutions and test parameters, related to the variables associated with the QD construct solution development process, that were investigated in the first round of keratinocyte experiments are given in Table 4.1. Figure 4.1 shows representative images of the four-well slides, each containing four culture chambers, used in R1 and R2. 


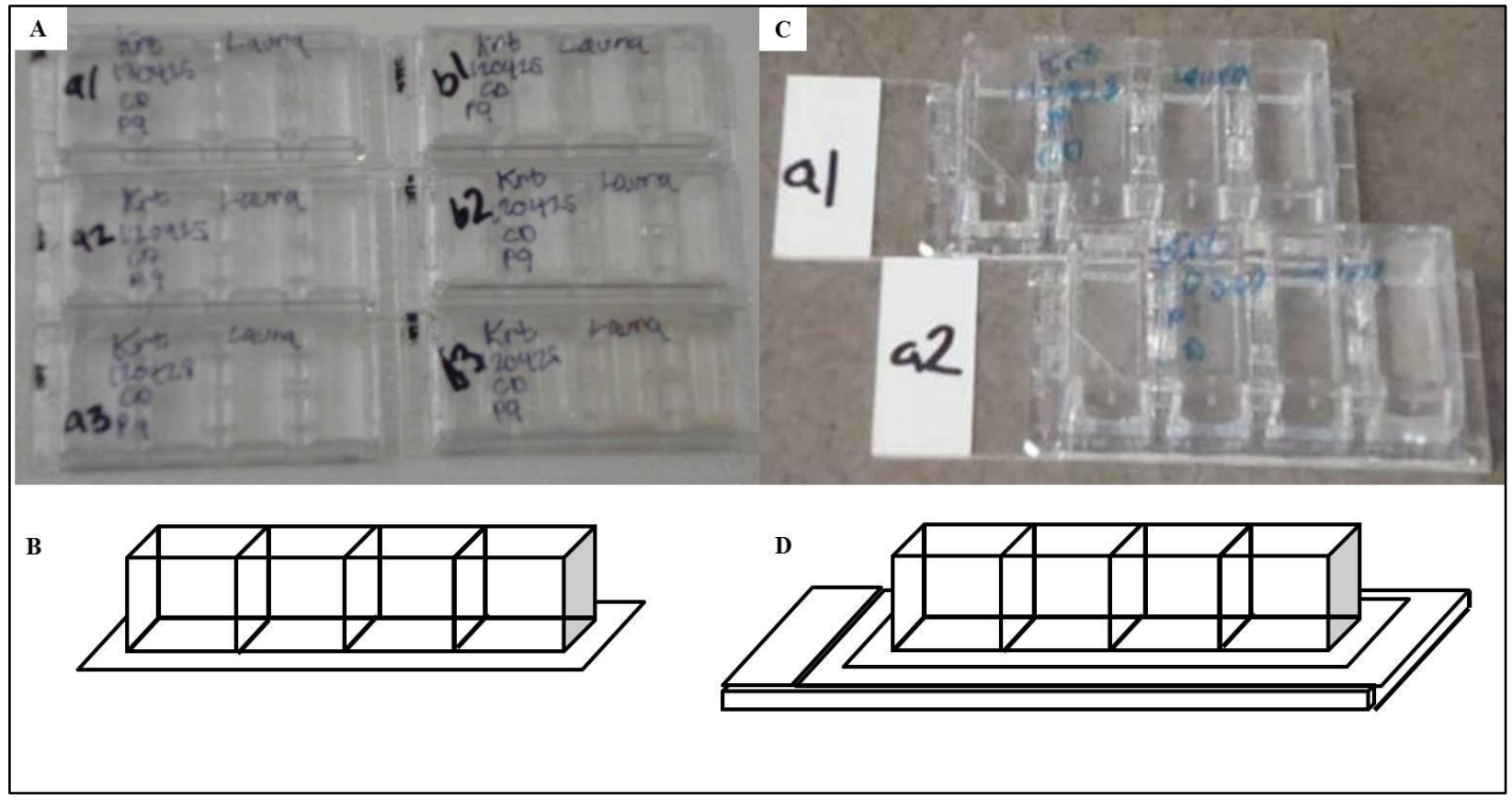

Figure 4.1 Representations of four-well slide configurations. A. All six four-well slides used in R1 of keratinocyte trials. B. Schematic diagram of four-well slide without thick specimen slide base. C. Four-well slides used in R2. D. Schematic diagram of four-well slide with thick specimen slide base.

Each well-set used in the first round of the keratinocyte trials, $\mathbf{a}$ and $\mathbf{b}$, had three four-well slides for a total of 12 wells in each set. Within each set, $\mathbf{a}$ and $\mathbf{b}$, were three sub-sets, one for each slide. Slide one of set a contained wells a1.1-4, slide two contained wells a2.5-8, and slide 3 contained wells a3.9-12. This same labeling was applied to well-set $\mathbf{b}$. Table 4.1 shows the test solutions corresponding to each well, the size of the filter that may have been used to filter the test solutions, and the fluorescent stain applied each well. The designation L/D represents the live/dead stain, which was composed of Calcein and Ethidium homodimer-1. The first round of keratinocyte trial test solutions were created primarily to determine the extent to which each step in the QD construct synthesis process affected cell health. Heating the keratinocyte media to $55^{\circ} \mathrm{C}$, exposure of the keratinocyte media to chloroform during evaporation, the presence and size of phospholipid particulates, and filtering to reduce contamination were all variables that could affect change on 
cell viability and proliferation. Therefore, each well was filled with a test solution that corresponded to the variable in question.

Table 4.1 Summary of Round One QD Construct Control and Test Solutions

\begin{tabular}{|c|c|c|c|c|}
\hline Well & Test Solution & $\begin{array}{c}\text { Heated } \\
55^{\circ} \mathrm{C}\end{array}$ & Filter & Stain \\
\hline a, b1.1 & Control - krt media & & & $\mathrm{L} / \mathrm{D}$ \\
\hline a, b1.2 & Control - krt media & $\mathrm{X}$ & & $\mathrm{L} / \mathrm{D}$ \\
\hline a, b1.3 & Control - krt media post-CHCl3 & $\mathrm{X}$ & & $\mathrm{L} / \mathrm{D}$ \\
\hline a, b1.4 & Control - krt media/PL post-CHCl3 & $\mathrm{X}$ & & $\mathrm{L} / \mathrm{D}$ \\
\hline a, b2.5 & $\mathrm{PL} / \mathrm{krt}[10 \mathrm{mg} / \mathrm{mL}]$ & $\mathrm{X}$ & $0.45 \mu \mathrm{m}$ & $\mathrm{L} / \mathrm{D}$ \\
\hline a, b2.6 & $\mathrm{PL} / \mathrm{krt}[10 \mathrm{mg} / \mathrm{mL}]$ & $\mathrm{X}$ & $0.45 \mu \mathrm{m}$ & $\mathrm{L} / \mathrm{D}$ \\
\hline $\mathbf{a}, \mathbf{b 2 . 7}$ & $\mathrm{PL} / \mathrm{krt}[10 \mathrm{mg} / \mathrm{mL}]$ & $\mathrm{X}$ & & $\mathrm{L} / \mathrm{D}$ \\
\hline a, b2.8 & $\mathrm{PL} / \mathrm{krt}[10 \mathrm{mg} / \mathrm{mL}]$ & $\mathrm{X}$ & & $\mathrm{L} / \mathrm{D}$ \\
\hline a, b3.9 & 42612wEN.2a: PL/krt [10mg/mL] & $\mathrm{X}$ & $0.45 \mu \mathrm{m}$ & $\mathrm{L} / \mathrm{D}$ \\
\hline a, b3.10 & 42612wEN.2a: PL/krt [10mg/mL] & $\mathrm{X}$ & $0.45 \mu \mathrm{m}$ & $\mathrm{L} / \mathrm{D}$ \\
\hline a, b3.11 & 42612wEN.2a: PL/krt [10mg/mL] & $\mathrm{X}$ & & $\mathrm{L} / \mathrm{D}$ \\
\hline a, b3.12 & 42612wEN.2a: PL/krt [10mg/mL] & $\mathrm{X}$ & & $\mathrm{L} / \mathrm{D}$ \\
\hline
\end{tabular}

Four variations of keratinocyte media, applied to wells a, b1.1-1.4, were used as a set of controls. Wells a,b1.1 were used as a primary control and wells a,b1.2-4 were used as secondary controls. The secondary controls were compared with the primary control, as well as with the unadulterated keratinocyte media, to determine the influence of the heat, chloroform exposure, and the presence of phospholipids on the media's ability to function in the expected capacity of cell maintenance. The results of the primary control, as well as that of the secondary controls, were used as a baseline for cell viability. The controls were used as a standard of comparison during assessment of wells a, b2.5-8 and a, b3.9-12, both of which contained additional components.

After attending to the issue of which effects, related to alterations of the keratinocyte media during the QD construct synthesis process, had an influence on cell viability, two additional elements needed to be addressed with respect to the test solutions: particle size and culture contamination. In wells a,b2.5-8, containing only phospholipids in media, the questions 
investigated pertained to what role the size of the particles played in the interaction between the constructs and the cells and what degree of contamination could the test solutions introduce into the cell cultures. It was important to determine if the presence of phospholipids alone had a negative effect on the cells' viability and if so, was it merely because they introduced some phospholipidspecific factor into the environment or were the negative effects the result of the phospholipids directly interacting with the cells. A $0.45 \mu \mathrm{m}$ filter was used to acquire these answers. Filtering was also applied to two of the four solutions containing the phospholipid encapsulated QDs. Filtering of the QD construct solutions was not only done for the previously stated reasons, but also to determine the effect of filtering on the CMW, intensity, and FWHM of the fluorescence emission of the solution.

The initial CdSe core sample, 42612wCMBO.2, used in the first step for creating the QD construct test solutions, was a combination of five previously synthesized CdSe core samples that had originally been synthesized using the modified protocol in Chapter 2. A ZnS coating was applied to this combination sample using a shelling temperature of $160^{\circ} \mathrm{C}$ and producing the CdSe/ZnS QD sample labeled 42612wQD.2a. The encapsulation process documented in Chapter 3 was then applied to the newly shelled sample to create the hydrophilic QD constructs and transfer them into the keratinocyte media. After encapsulation the QD construct, the solution was passed through a 0.45 micron filter to aid in removal of any contaminants present within the solution, as well as to remove any excess phospholipids that had failed to form a micelle around the QDs. At each step during the QD construct synthesis a fluorescence spectra was taken using the Ocean Optics USB 4000 fiber optic spectrometer to track the changes in CMW, intensity, FWHM, and scattering resulting from the processes and materials of each step. 


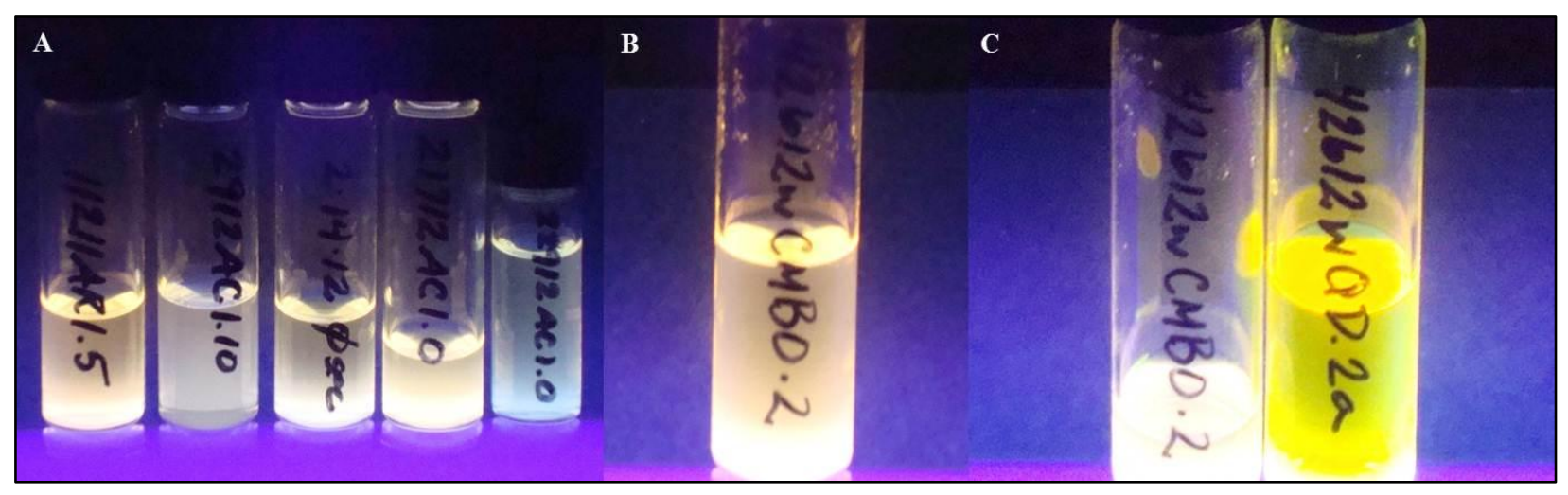

Figure 4.2 Progression of the 42612wQD.2a used in the test solutions in the first round of keratinocyte QD construct trials. A. The five individual CdSe composite cores. B.The five combined CdSe cores. C. The combined CdSe cores shelled with $\mathrm{ZnS}$ at $160^{\circ} \mathrm{C}$ (550nm: 2800 counts)

\subsubsection{QD Construct Control and Test Solutions - R2}

Round two of the test solution experiments concentrated on assessing the effects of added filtering with 0.2 micron diameter pore filters, as well as using additional staining techniques to assess cell viability and cell-QD construct interaction. Additional filtering, using a $0.2 \mu \mathrm{m}$ filter, was imposed to investigate the benefits of using a more rigorous filtering regime. The expected benefits of using a smaller filter were a decreased incidence of contaminating components within the solution and size selection of the QD constructs suspended in solution. Decreasing the contaminants within the solution would prevent the growth of non-cellular components within the culture. Reducing the size of the QD constructs could promote the attachment and integration of the constructs to the keratinocyte cells. The test solutions, the filters employed, and the stains applied to each well-set are given in Table 4.2. Like the test solutions used in R1, the well designations indicate each four-well slide and each sub-set of wells. In the second round of keratinocyte trials a second staining technique, Histochoice and Hoechst, was employed for additional assessment of cell presence and proliferation. Two different QD constructs were used in the test solutions: 52312EN45.60b and 42612oEN.1a. 
Table 4.2 Summary of Round Two QD Construct Control and Test Solutions

\begin{tabular}{|c|c|c|c|c|c|}
\hline Well & Test Solution & Filter & $\begin{array}{l}\text { Stain } \\
\text { (a) }\end{array}$ & $\begin{array}{c}\text { Stain } \\
\text { (b) }\end{array}$ & $\begin{array}{l}\text { Stain } \\
\text { (c) }\end{array}$ \\
\hline a, b, c1.1 & Control - krt media & & $\mathrm{H} / \mathrm{H}$ & $\mathrm{L} / \mathrm{D}$ & $\mathrm{H} / \mathrm{H}$ \\
\hline $\mathbf{a}, \mathbf{b}, \mathbf{c 1 . 2}$ & 42612oEN.1a: PL/krt $[10 \mathrm{mg} / \mathrm{mL}]$ & $0.2 \mu \mathrm{m}$ & $\mathrm{H} / \mathrm{H}$ & $\mathrm{L} / \mathrm{D}$ & $\mathrm{H} / \mathrm{H}$ \\
\hline $\mathbf{a}, \mathbf{b}, \mathbf{c 1 . 3}$ & {$[10 \mathrm{mg} / \mathrm{mL}]$} & $0.2 \mu \mathrm{m}$ & $\mathrm{H} / \mathrm{H}$ & $\mathrm{L} / \mathrm{D}$ & $\mathrm{H} / \mathrm{H}$ \\
\hline$a, b, c 1.4$ & 52312EN45.60b: PL/krt [10mg/mL] & $0.2 \mu \mathrm{m}$ & $\mathrm{H} / \mathrm{H}$ & $\mathrm{L} / \mathrm{D}$ & $\mathrm{H} / \mathrm{H}$ \\
\hline$a, b, c 2.5$ & 42612oEN.1a: PL/krt $\quad[10 \mathrm{mg} / \mathrm{mL}]$ & $0.45 \mu \mathrm{m}$ & $\mathrm{L} / \mathrm{D}$ & $\mathrm{H} / \mathrm{H}$ & $\mathrm{L} / \mathrm{D}$ \\
\hline a, b, c2.6 & Control - krt media & & L/D & $\mathrm{H} / \mathrm{H}$ & L/D \\
\hline a, b, c2.7 & 52312EN45.60b: PL/krt [10mg/mL] & $0.45 \mu \mathrm{m}$ & $\mathrm{L} / \mathrm{D}$ & $\mathrm{H} / \mathrm{H}$ & $\mathrm{L} / \mathrm{D}$ \\
\hline $\mathrm{a}, \mathrm{b}, \mathrm{c} 2.8$ & {$[10 \mathrm{mg} / \mathrm{mL}]$} & $0.45 \mu \mathrm{m}$ & $\mathrm{L} / \mathrm{D}$ & $\mathrm{H} / \mathrm{H}$ & $\mathrm{L} / \mathrm{D}$ \\
\hline
\end{tabular}

Two different QD construct samples were used as test solutions: 42612oEN.1a and 52312EN45.60b. The original CdSe core sample used to synthesize 42612oEN.1a was composed of four previous CdSe samples: 102511W2.10, 12611AC.30, 2812ARC.1A, and 2812ARC.1B0. This new combination sample, $42612 \mathrm{oCMBO} .1$, had been shelled with a $\mathrm{ZnS}$ coating at $160^{\circ} \mathrm{C}$, encapsulated in a $10 \mathrm{mg} / \mathrm{mL} \mathrm{PL} / \mathrm{CHCl}_{3}$ solution, and evaporated into keratinocyte media using the protocol from Chapter 3.

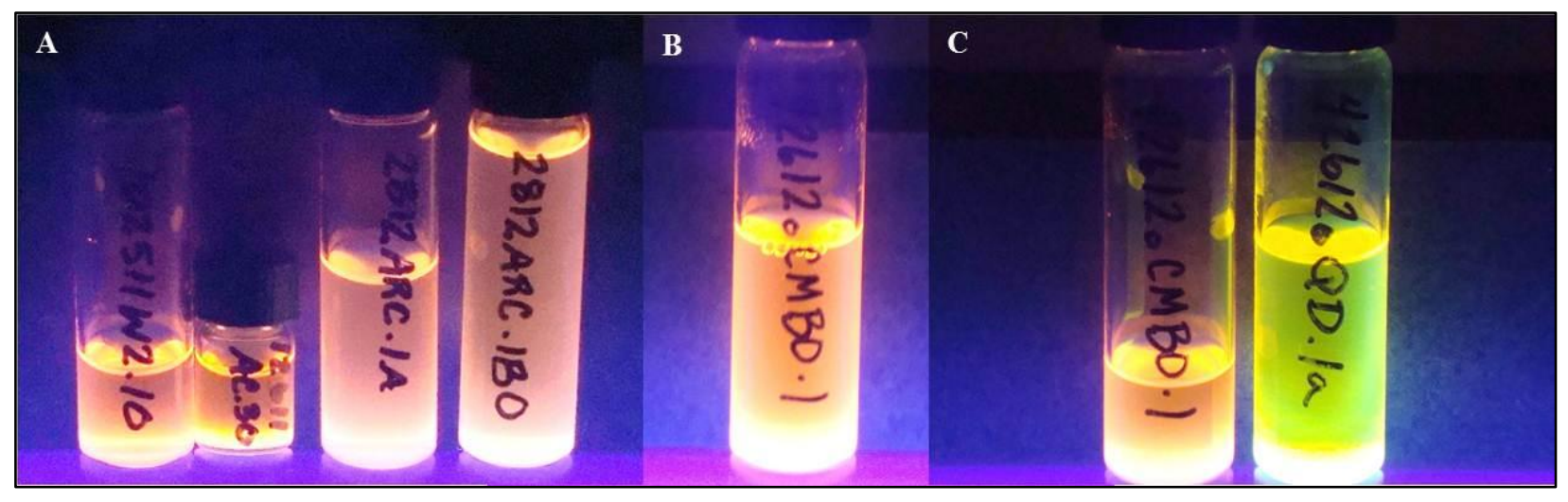

Figure 4.3 Progression of the 42612oEN.1a used in the test solutions of R2 trials. A. The four individual CdSe composite cores. B. The four combined CdSe cores. C. The combined CdSe cores shelled with $\mathrm{ZnS}$ at $160^{\circ} \mathrm{C}$ (537nm:7400counts: FWHM 40). 
The original CdSe core sample used to synthesize 52312EN45.60b was a combination of two previous CdSe samples, 52312AC4.60 and 52312AC5.60, both which were synthesized at $225^{\circ}$, combined, shelled with a $\mathrm{ZnS}$ coating at $110^{\circ} \mathrm{C}$, encapsulated in a $10 \mathrm{mg} / \mathrm{mL} \mathrm{PL} / \mathrm{CHCl}_{3}$ solution, and evaporated into keratinocyte media using the protocol from Chapter 3 . The choice of each $\mathrm{CdSe} / \mathrm{ZnS}$ sample to be used in the synthesis of the QD construct test solutions was made based on the CMW and FWHM of each sample relative to the emission wavelengths associated with the L/D stains; it was necessary that the emission wavelength of the QD constructs overlap as little as possible with the L/D stain emission wavelengths. With the CMW of the emission wavelengths for Calcein and EthD-1 being 515 and $635 \mathrm{~nm}$ respectively, it was important to have a QD emission wavelength that would be intermediate between the two with as little overlap as possible. The CMW, intensity, and FWHM of 42612oEN.1a and 52312EN45.60b were 537nm: 7400counts: FWHM 40nm and 564nm: 15200counts: FWHM 44nm respectively.

In R2 of the keratinocyte trials the NFK cells were thawed out of cryopreservation state and cultured in T75 flasks. Three days later the cells were passed into 6 four-well slides. As a consequence of not having the same four-well slides available that were used in the first round of keratinocyte trials, the keratinocytes were passed into the alternate four-well slides with the thick specimen slide base. As a result of low observed confluency, additional cells, from the initial T75 flasks, were added to the 6 four-well flasks on 3 days after the first passage into the 6 four-well slides. Three feedings of keratinocyte media were administered in the interim between the initial thawing and the addition of cells. The 6 four-well slides were divided into 3 sets: a, b, and $\mathbf{c}$. Each well-set was examined with the optical microscope before the introduction of the test solutions and at their individual time points post-introduction. Conditions in well-set a were scheduled to be investigated one hour after the introduction of the test solutions, conditions in well-set $\mathbf{b}$ at 12 hour post-introduction, and well-set c 24 hours post introduction of the test solutions. Although the time 
points for investigating the conditions in well-sets $\mathbf{b}$ and $\mathbf{c}$ did follow the initial schedule, the initial schedule for well-set a was altered to accommodate the hurdles of using the four-well slides mounted on the thick specimen slides bases, the missing Hoechst stain, and the lights in the engineering building that housed the confocal microscope being shut off previous to imaging well sub-set a2.5-8.

\subsubsection{Duration of Keratinocyte Trial $-R 1$}

The first round of keratinocyte trials explored two different time-frames. Well-set a examined the cell-QD construct interaction and effect of the test solutions on cell viability and proliferation for a 24 hour exposure time and well-set b explored the cell-QD construct interaction and effect of the test solutions on cell viability and proliferation for a 24 hour exposure time with an additional latent period of 24 hours in fresh media. In other words, well-set $\mathbf{b}$ underwent the same initial conditions as well-set a, but after the initial 24 hours of exposure, the test solutions were aspirated off, fresh media was added, and another 24 hours of incubation time was initiated. The purpose of this tactic was to assess the longer term consequences of the cells to a discrete exposure of the test solutions.

The cells were removed from the primary aseptically maintained incubator and examined under a white light microscope. Observations of cell confluency and morphology, and the presence of any contaminants, were recorded and representative images of each well-chamber were taken using a Sony Cyber-Shot digital camera on low light high sensitivity setting at maximum zoom without a flash. After imaging the cells pre-introduction of the test solutions was completed, the choice to aspirate or not to aspirate was made. The decision to aspirate off the initial media present in the cultures before introduction of the test solutions was dependent upon three factors: the time relative to the most recent feeding, the amount of media residing in each of the culture wells, and the desired dilution of the test solutions. If the last feeding was more than 24 hours prior to 
introduction of the test solutions, the initial media was aspirated out of the wells and the appropriate amount of media, relative to the desired dilution of the test solutions, was added. If the time between the introduction of the test solutions and the most recent feeding did not necessitate new media but the wells were full, and therefore did not have room to add the test solutions, the media was aspirated off. Ideally, enough media would be available to support the decision to aspirate off the initial media, add fresh media to the test solutions achieving the desired dilution, and then add the test solutions to the cell cultures. After the media was aspirated off, using a motor powered aspiration system, the test solutions were added to each well, and the cultures were then placed in the secondary incubator and maintained at a temperature of $37^{\circ} \mathrm{C}$.

Twenty four hours after the introduction of the test solutions both well-set $\mathbf{a}$ and well-set $\mathbf{b}$ were removed from the secondary incubator and all wells were aspirated, removing the test solutions. Each well of well-set $\mathbf{b}$ was then rinsed with $0.5 \mathrm{ml}$ of keratinocyte media, aspirated, filled with $3 \mathrm{~mL}$ of keratinocyte media, re-imaged, and placed back into the secondary incubator. Observations of cell confluency and morphology, and the presence of any contaminants were recorded with corresponding representative images for each well-chamber. These images were later compared with the corresponding confocal images. $1.5 \mathrm{~mL}$ of keratinocyte culture media was added to each well of well-set a and the cultures were again examined under the microscope; no images of well-set a were taken at this time.

\subsubsection{Duration of Keratinocyte Trial $-R 2$}

Three different time points were investigated in the second round of trials: one hour, 12 hours, and 24 hours post-introduction of test solutions. The purpose of these time points was twofold: to determine the proportion of cell damage relative to time of exposure and to determine the rate of attachment and of QD constructs to cell membranes. Each four-well slide was removed from the primary incubator and examined using the optical white light microscope. As a result of 
diminished stores of fresh keratinocyte media on hand, the existing media in each of the wells, approximately $1 \mathrm{~mL}$, was not removed. All test solutions were introduced to the cultures, $1.5 \mathrm{~mL}$ per well, and the slides were placed in the secondary incubator at $37^{\circ} \mathrm{C}$. The $\mathrm{L} / \mathrm{D}$ stain solution was made during the one hour waiting period for the first set of slides to incubate. The Hoechst dye material was unavailable for use in well-set $\mathbf{a}$ and was therefore not implemented until testing of well-set $\mathbf{b}$. At one hour from introduction of the test solutions well-set $\mathbf{a}$ was removed from the secondary incubator.

Table 4.3 Keratinocyte Trial Time Points in Round Two

\begin{tabular}{|c|c|c|c|c|c|}
\hline $\begin{array}{c}\text { well- } \\
\text { set }\end{array}$ & $\begin{array}{c}72 \text { hours post- } \\
\text { passing }\end{array}$ & hour 0 & hour 1 & hour 12 & hour 24 \\
\hline a & $\begin{array}{c}\text { image with optical } \\
\text { microscope \& } \\
\text { digital camera }\end{array}$ & $\begin{array}{c}\text { introduce } \\
\text { test } \\
\text { samples }\end{array}$ & $\begin{array}{c}\text { add L/D \& H/H } \\
\text { stains, image with } \\
\text { confocal }\end{array}$ & $\mathrm{X}$ & $\mathrm{X}$ \\
\hline b & $\begin{array}{c}\text { image with optical } \\
\text { microscope \& } \\
\text { digital camera }\end{array}$ & $\begin{array}{c}\text { introduce } \\
\text { test } \\
\text { samples }\end{array}$ & incubate & $\begin{array}{c}\text { add L/D \& H/H } \\
\text { stains, image with } \\
\text { confocal }\end{array}$ & $\mathrm{X}$ \\
\hline c & $\begin{array}{c}\text { image with optical } \\
\text { microscope \& } \\
\text { digital camera }\end{array}$ & $\begin{array}{c}\text { introduce } \\
\text { test } \\
\text { samples }\end{array}$ & incubate & incubate & $\begin{array}{c}\text { add L/D \& H/H } \\
\text { stains, image with } \\
\text { confocal }\end{array}$ \\
\hline
\end{tabular}

\subsubsection{Implementation of White Light Optical Imaging}

At the onset of each trial, the previously prepared four-well flasks were removed from the primary incubator and viewed before adding the test solutions. Each well of keratinocyte cell cultures was examined using an Olympus CKX41 optical white light microscope at magnifications of both $10 \mathrm{X}$ and $20 \mathrm{X}$ with the intermediate light aperture. Examination of the cells was performed to qualitatively classify the general appearance and morphology of the cells within the culture, both as individual cells and as a culture population. Individual cell morphologies were examined by assessing cell shape, size, and adhesion according to the characteristics as described by the 
distributor of the keratinocyte cells, ATCC Primary Cell Solutions. The expected morphology, developmental phase, and distribution, indicative of healthy cells, are a cobblestone appearance, high mitotic index, colony formation of cells when at near $80 \%$ confluency, and cell adherence to the well-plate surface (81). A cobblestone appearance is characterized by individual cells that are rounded not flat. Mitotic Index refers to the ratio of cells within the culture population that are actively undergoing mitosis relative to cells not undergoing mitosis (82). The keratinocyte cell culture images in Figure 4.4 show these representation fundamental keratinocyte culture characteristics at four different percentages of confluency. In addition to these fundamental cell traits, any anomalies found within each well were also assessed and recorded to be later referenced in the case of unexpected developments within the cultures at a later time point.

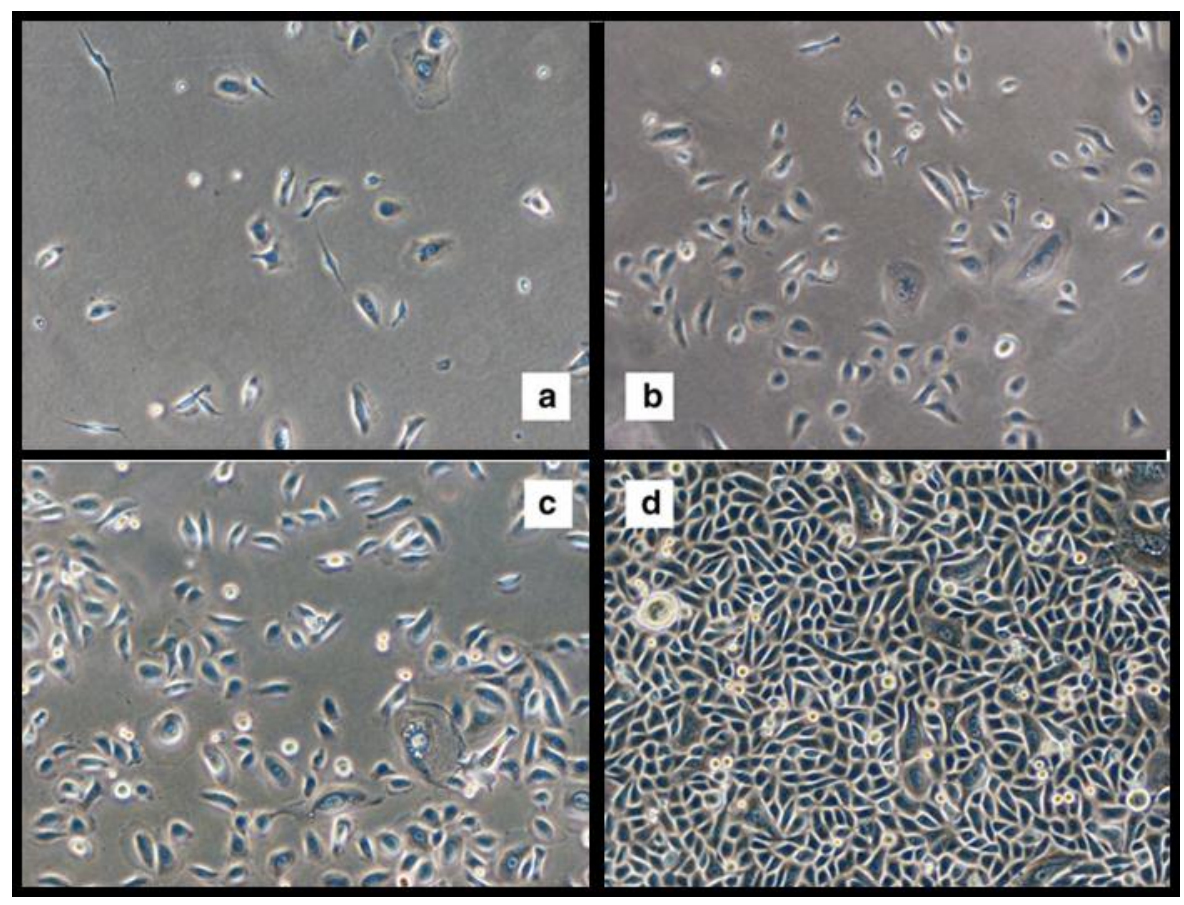

Figure 4.4 Microscopic view of neonatal foreskin keratinocytes (NFK) at different stages of proliferation and colonization: a. initiation of NFK cells, day 8 after seeding; $\mathbf{b}$. growing colonies at day $11 ; \mathbf{c}$ day $15 ; \mathbf{d}$. confluent area at day $20(83)$. 


\subsubsection{Optical Imaging $-R I$}

A minimum of three initial images were taken for each well in both well-set $\mathbf{a}$ and well-set b. Multiple images of each well were taken to ensure that an accurate representation of the state of the whole culture was recorded pre-introduction of the test solutions. These first images were taken with the initial media still present in each well. The purpose of imaging before removing the initial media was to ensure that any dead cells or contaminants contained within the media would be included in the initial observations. Had the initial media been aspirated off before imaging, these abnormalities would have gone unidentified. The wells were then aspirated and the test solutions were then added to both well-sets, $1.5 \mathrm{~mL}$ per well without additional fresh media, and both wellsets were placed back into the secondary incubator. At the 24 hour mark well-set $\mathbf{b}$ was removed from the secondary incubator, the test solution in each well was aspirated off, $1.5 \mathrm{~mL}$ of fresh media was added, and another set of images were taken using the white light optical microscope. After the representative images were taken, well-set $\mathbf{b}$ was placed back into the incubator for a final period of 24 hours. At this time, after well-set $\mathbf{b}$ was returned to the incubator, the test solutions were aspirated off of well-set a. After aspiration of the test solutions $0.5 \mathrm{~mL}$ of keratinocyte was added to each well and used to rinse off any remaining test solution. The rinse was then aspirated off and a second volume of $0.5 \mathrm{~mL}$ of keratinocyte media was added. This additional $0.5 \mathrm{~mL}$ of media was added as part of the preparation for staining the cells with the Calcein/EthD-1 Live/Dead (L/D) stain. No images of well-set a were taken after aspiration of the test solutions. Forty eight hours after the initial, 24 hours after well-set $\mathbf{b}$ had been returned to the secondary incubator with fresh media, the optical microscope was once again used to image each well in well-set $\mathbf{b}$. Through visual observation it was determined that little to no change had occurred in the cells during the 24 hours since the last viewing and therefore no additional white light images of well-set $\mathbf{b}$ were 
taken. To more clearly illustrate the time line of test solution introduction and imaging, Figure 4.5 gives the sequence of events in detail.

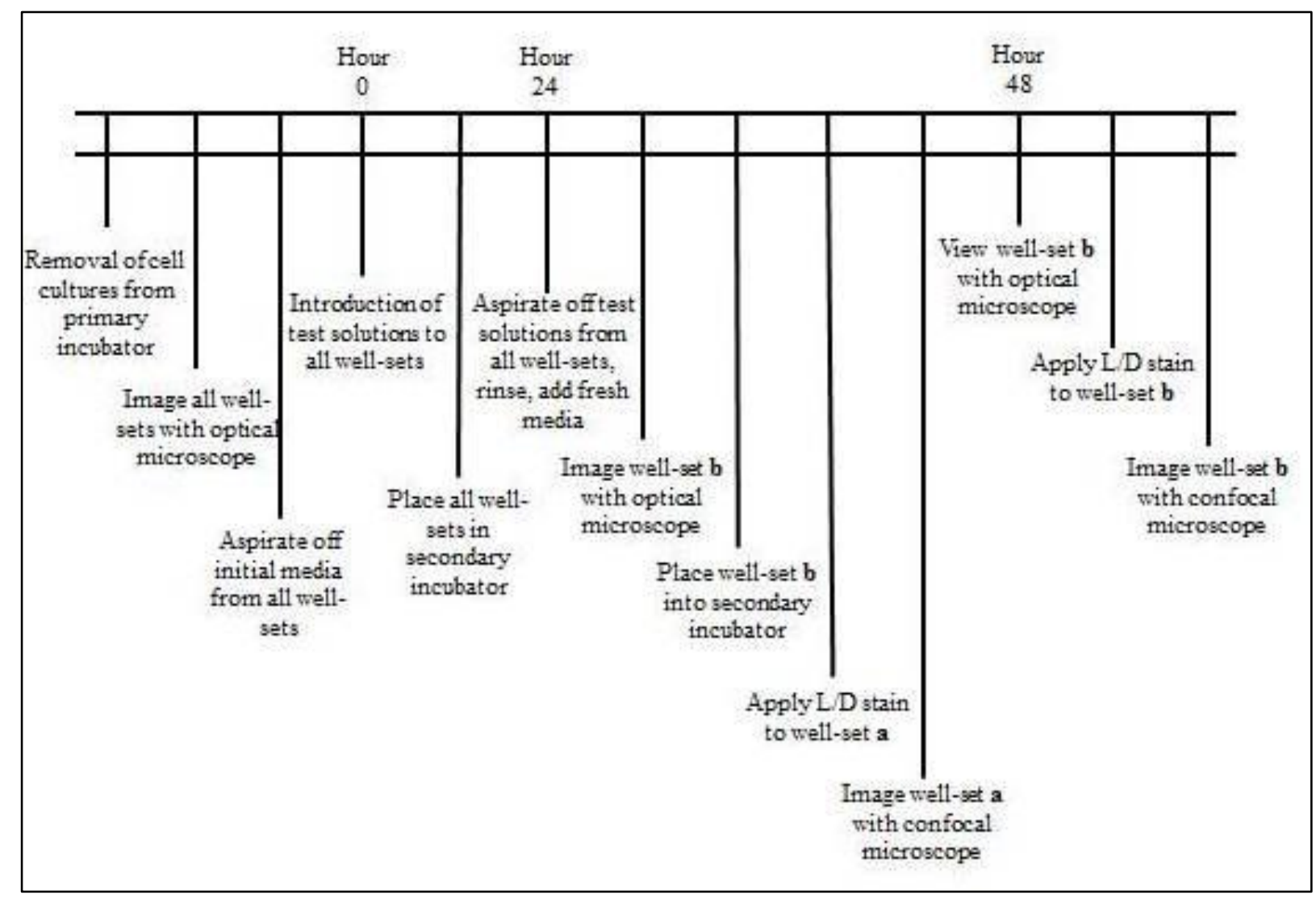

Figure 4.5 Sequence of Cell Culture - Test Solution Interaction and Imaging

\subsubsection{Optical Imaging $-R 2$}

The pattern of imaging each well-set in the second round of trials was similar to that of the pattern of imaging for the first round of trials, but in this case all well-sets were imaged both before and after the introduction of the test solutions. All three well-sets, $\mathbf{a}, \mathbf{b}$, and $\mathbf{c}$, were removed from the primary incubator and imaged, before introduction of the test solutions, with the initial media still in each well. After imaging each well, and recording all pertinent observations, $1.5 \mathrm{~mL}$ of the test solution corresponding to each well was added. Unlike the first round of trials the initial media 
was not aspirated off before the introduction of the test solutions. Not aspirating off the initial media before introduction of the test solutions was the result of having only a limited supply of keratinocyte available. All of the well-sets were then placed back into the secondary incubator. At one hour after the introduction of the test solutions well-set a was aspirated, rinsed with $0.5 \mathrm{~mL}$ of keratinocyte media, aspirated, rinsed with $0.5 \mathrm{~mL}$ of keratinocyte media, and then re-imaged with the optical microscope before introducing any fluorescence staining. This same series of rinsing with the stain-associated media, aspirating, and imaging was applied to well-sets $\mathbf{b}$ and $\mathbf{c}$ at 12 and 24 hours respectively.

\subsubsection{Fluorescence Staining}

Two varieties of fluorescent staining methods were employed for determining the cell viability of the keratinocyte cultures post-introduction of test solutions and the degree of cell-QD construct interaction: Calcein/Ethidium homodimer-1 (EthD-1) Live/Dead cell viability stain (L/D) and Histochoice/Hoechst $(\mathrm{H} / \mathrm{H})$ nuclei stain. The application of the L/D stain was used to simultaneously test two separate parameters of cell viability: intracellular esterase activity and plasma membrane integrity (84). It is the esterase activity inherent in all live cells that acts as a catalyst causing a conversion of the non-fluorescing calcein AM in the L/D stain solution to the green fluorescing species calcein (84). As the period of time that a cell has been dead increases, less and less calcien AM is available to be converted into calcein, resulting in a corresponding decrease of fluorescence. Although the presence of the calcein associated green fluorescence isn't a negative for dead cells, it does indicate that at the time of the introduction of the L/D stain there was still intracellular esterase activity in the cell; an indication that the cell was most likely alive at the time of the introduction (85). EthD-1 is used to determine the presence of dead cells in the culture. EthD-1 can only enter cells with damaged membranes. Previous to entering the cells the level of EthD-1 is low enough to be hidden in the background noise. It is only upon invasion in to 
the cell through the permeable membrane, and binding to the nucleic acids within the cell, that the EthD-1's red fluorescence signature is observed (84). Any amount of red fluorescence in a cell is an indication that the cell's membrane has become permeable as a result of cell death. A cell that fluoresces almost entirely green but that does exhibit some red fluorescence is an indication of recent cell death; possibly as a direct result contact with the L/D stain. A cell that fluoresces almost entirely red is an indication that cell death was not recent; most probably a result of environmental factors unrelated to the L/D stain. The excitation wavelength for both calcein and EthD-1 is $495 \mathrm{~nm}$, with calcein having and emission wavelength of 515nm and EthD-1 having an emission wavelength of $635 \mathrm{~nm}$. The $\mathrm{L} / \mathrm{D}$ stain solution applied to both $\mathrm{R} 1$ and $\mathrm{R} 2$ trials was made using Invitrogen's Live/Dead Viability/Cytotoxicity Kit.
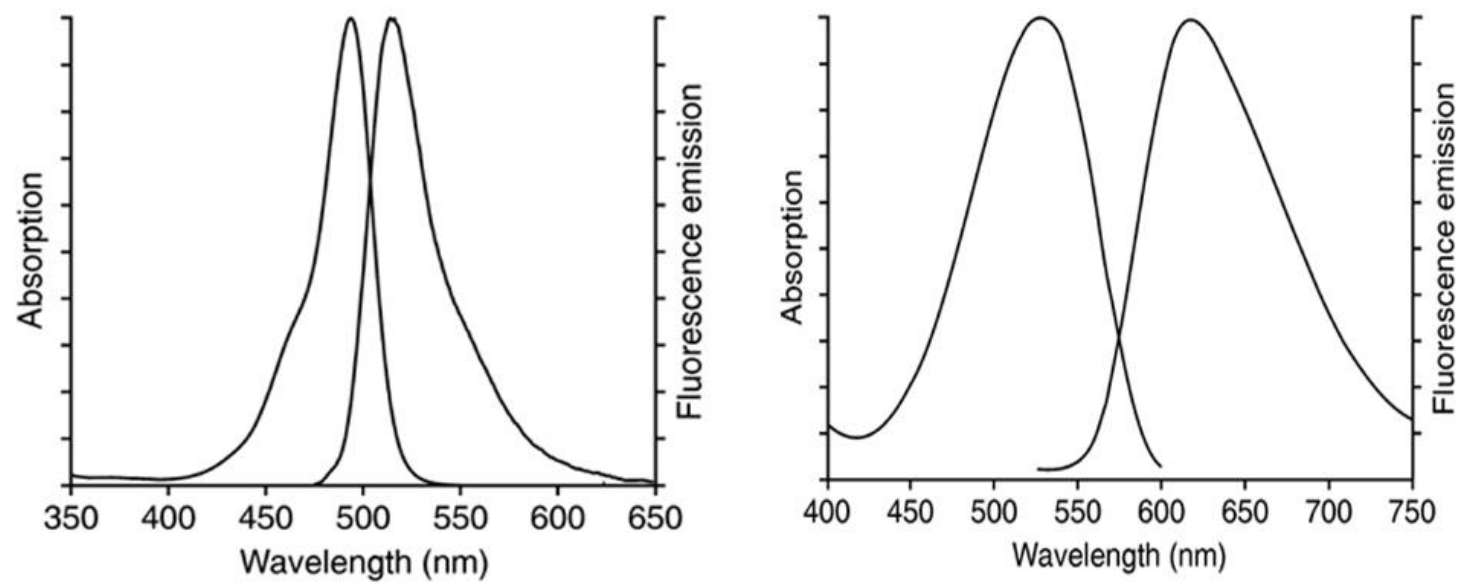

Figure 4.6 On the left is the excitation and emission fluorescence spectra of Calcein (495/515), on the right is the excitation and emission spectra of EthD-1 (495/635) $(86,87)$.

The Hoechst stain used in the R2 trials was Invitrogen's Hoechst 34580. This particular variety of Hoechst has can be excited using a 405nm wavelength laser and is therefore appropriate for use when imaging with a confocal microscope. Hoechst stains are cell permeable nucleic acid stains (83). Hoechst 34580 was used in this thesis to positively confirm the presence of 
keratinocyte cell nucleuses from other biologic entities that may have been present in the cultures and which may have reacted with the L/D stain and QD constructs in R1, but were not in fact keratinocyte cells.

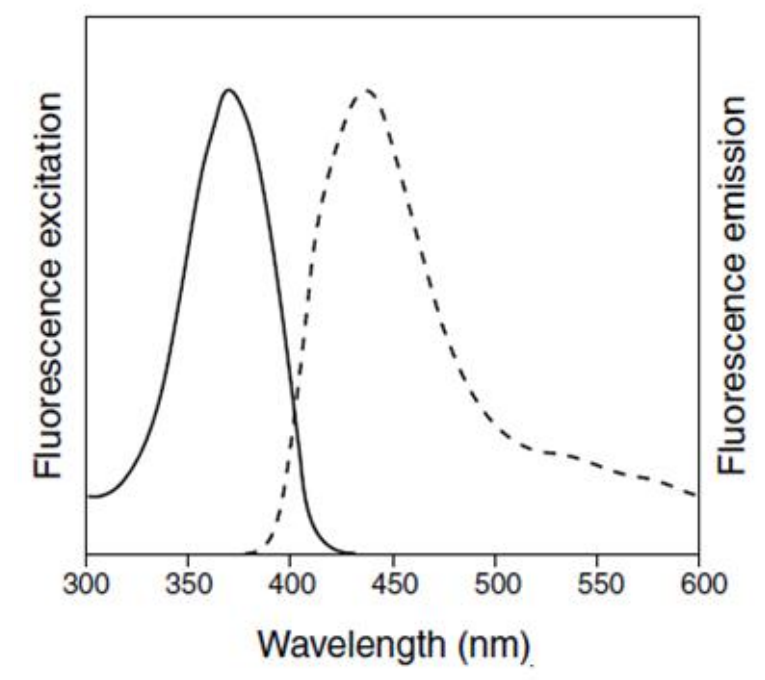

Figure 3. Fluorescence excitation and emission spectra of Hoechst 34580 bound to DNA.

Figure 4.7 The excitation (solid line) and emission (dotted line) fluorescence spectra of Invitrogen's Hoechst 34580 stain (88).

\subsubsection{Calcein/EthD-1 Live/Dead Cell Viability Stain - Rland R2}

In $\mathrm{R} 1$, as well as $\mathrm{R} 2$, the $\mathrm{L} / \mathrm{D}$ stain was made by mixing $20 \mu \mathrm{L}$ of $2 \mathrm{mM}$ EthD-1, $10 \mathrm{~mL}$ of keratinocyte media, and $5 \mu \mathrm{L}$ of $4 \mathrm{mM}$ Calcein in a $20 \mathrm{~mL}$ glass vial. The solution was vortexed and the glass vial was wrapped in aluminum foil to prevent a reduction in the stains efficacy resulting from over exposure to light. For both rounds of keratinocyte trials the L/D stain was prepared and applied to the cultures in the same manner and in the same time frame. The well sub-set to be imaged was aspirated to remove the test solutions, rinsed with $1.5 \mathrm{~mL}$ of keratinocyte media, aspirated again, and another $1.5 \mathrm{~mL}$ of keratinocyte media was added. The entire protocol for the preparation and use of the L/D stain is given in Appendix B.9. The L/D stain was not added to the cell cultures until 10 minutes before imaging with the confocal microscope. This delay in 
administering the L/D stain was used as a measure for decreasing the influence that the stain had on the health of the cells.

\subsubsection{Histochoice/Hoechst Nucleus Stain - R2}

The Hoechst stain was applied in the R2 trials only. The protocol used for the preparation of the Hoechst stain was originally created for staining coverslips coated with NIH 3T3 fibroblast cells. This original protocol of the Hoechst stain called for preparation of a $2 \mu \mathrm{g} / \mathrm{mL}$ concentration solution of Hoechst dye using a $5 \mathrm{mg} / \mathrm{mL}$ stock solution and applying $200 \mu \mathrm{L}$ of the solution to each coverslip. This concentration could be achieved by mixing $2 \mu \mathrm{L}$ of the stock solution with $5 \mathrm{~mL}$ of PBS. For the preparation of the Hoechst stain for use in the four-well slides of keratinocyte cultures, alterations were made to the original protocol. The Hoechst starting material on hand was in powder form, not liquid, and therefore was not measured out by mixing $2 \mu \mathrm{L}$ of Hoechst with 5mL of PBS. Instead, Hoechst stain was prepared by adding $2-4$ particles of the Hoechst powder with $5 \mathrm{~mL}$ of PBS in a glass vial and vortexing the solution. The glass vial was then wrapped in aluminum foil to prevent a decrease in the effectiveness of the solution as a result of exposure to light.

Each well-set in R2 had been divided into two subsets: a, b, c1.1-4 and a, b, c2.5-8. In the casee of well-set a, well-subset a1.1-4 was to receive the Histochoice fixative and Hoechst stain $(\mathrm{H} / \mathrm{H})$, well-subset a2.5-8 was to receive the L/D stain. Each stain was to be prepared in a different aqueous medium, PBS for the $\mathrm{H} / \mathrm{H}$ and keratinocyte media for the $\mathrm{L} / \mathrm{D}$; therefore, each well-subset was to be rinsed with $0.5 \mathrm{~mL}$ of the media corresponding to the stain that would be administered to that particular subset. The same pattern of aspiration, imaging, and rinsing was to be applied to well-sets $\mathbf{b}$ and $\mathbf{c}$ at 12 and 24 hours respectively from the initial introduction of the test solutions into the well-sets. The proposed schedule of staining was administered as intended for well-sets $\mathbf{b}$ and c, however, well-set a did not receive the $\mathrm{H} / \mathrm{H}$ staining as a result of the Hoechst stain base 
material being unavailable; instead both sub-sets received L/D stain. Additionally, as a consequence of using the alternate four-well slides with the thick specimen slide base, sub-set a1.14 was exposed to the L/D for an extended period of time before imaging with the confocal microscope; sub-set a2.5-8 was imaged 24 hours after sub-set a1.1-4 as a consequence of the lights in the building being turned off just before the L/D stain was to be introduced.

\subsubsection{Confocal and DIC Imaging}

An Olympus Fluoview confocal microscope was used to acquire confocal and differential interference contrast (DIC) images. Confocal images of the cell cultures exposed to the L/D and $\mathrm{H} / \mathrm{H}$ stains were acquired by applying the Alexafluor 488 filter to detect the Calcein emission, the PI filter to detect the EthD-1 emission, the Qdot565 filter to detect the QD construct emission, and the DAPI filter to detect the Hoechst stain emission. In the cases of imaging cell viability in wells that contained QD constructs, it was not possible to employ both the L/D and QD filters simultaneously owing to the overlap between the excitation and emission wavelength ranges associated with each filter. In this circumstance separate images were taken of the same field of view and then compared side by side. DIC images provided topological information about the structure of the cell cultures in each well. Using DIC imaging in concert with confocal imaging offered an additional method for determining the relative position of the QD constructs with respect to the cells. DIC images were obtained by applying the TIC filter.

\subsubsection{Keratinocyte Cell Viability - R1}

The first round of keratinocyte trials investigated cell viability and cell-QD construct interaction using only L/D staining and confocal imaging of the wells. The L/D stain was applied to each well sub-set, placed in a closed container out of the light, and allowed to incubate for 10 minutes before imaging. Each four-well slide was placed securely above the $20 \mathrm{X}$ objective lens. Before attempting to acquire an image, the wide-field fluorescence laser was engaged and the 
sample viewed through the eyepiece while adjusting the vertical position of the four-well slide relative to the objective lens until a clear image was achieved. After achieving a clear image in this manner, scanning of the image using the confocal laser and appropriate filters was initiated. A strategy of acquiring multiple images, at different focal depths, taken of a single area while adjusting the intensity of the laser was applied. The amount of the excitation laser source incident on the sample was adjusted by increasing or decreasing the "laser \%" on the control panel. An additional adjustment made in an effort to achieve maximum clarity of the cells and minimum interference of background fluorescence was manipulation of the high voltage (HV) setting associated with each filter. Each well within both well-sets $\mathbf{a}$ and $\mathbf{b}$ were imaged with this strategy.

As a result of the distinct emission wavelength of the QD constructs, CMW of $539 \mathrm{~nm}$ with a FWHM of 44nm, it was possible to distinguish the QD constructs from the cells when viewing the wells with the L/D stain associated filters alone. For clarification that the particles presumed to be QD constructs were in fact QD constructs, images of the same field of view within the wells containing the QD construct test solutions were taken using the L/D filters and then again using only the Qdot565 filter. These images were then assessed in a side by side comparison.

\subsubsection{Keratinocyte Cell Viability $-R 2$}

The second round of keratinocyte trials investigated cell viability and cell-QD construct interaction using L/D staining, $\mathrm{H} / \mathrm{H}$ staining, and confocal and DIC imaging of the wells. As stated in Section 4.2.4.2, a consequence of using the alternate four-well slides with the thick specimen slide base, was that sub-set a1.1-4 was exposed to the L/D for an extended period of time before imaging with the confocal microscope. The particular difficulty associated with using the alternate four-well slides stemmed from the thickness of the specimen slide base. The added thickness, relative to the four-well slides used in R1, prevented clear and accurate imaging with the confocal microscope. To combat this, directly before imaging, the solution within each well was aspirated 
off and the four-well chamber frame was removed. Removal of the four-well chamber frame left behind the rubber seal that had attached the frame to the slide. The formation and height of the rubber seal was such that when a cover slip was placed over the area previously occupied by the frame, there was still a significant gap between the coverslip and the cells. This gap precluded acquiring clear images. This was remedied by removing the rubber seal. In the case of well sub-set a1.1-4, the seal was removed using a metal spatula and tweezers. Removal of the rubber seal for well sub-set a2.5-8 and well-sets $\mathbf{b}$ and $\mathbf{c}$ employed the use of a razor blade and tweezers. The progression of slide alterations is given in Figure 4.8.

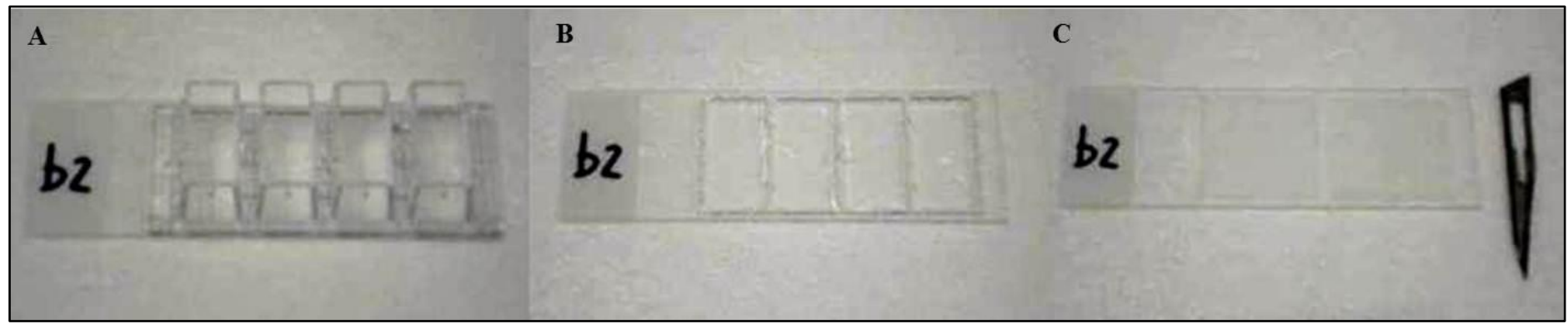

Figure 4.8 Adaptation of four-well with thick specimen slide base for use in confocal imaging of R2 keratinocyte trials. A. Four-well slide with well chambers. B. Four-well slide with rubber seal. C. Four-well slide ready for imaging.

As a result of the incompatibility of the Alexa Fluor 488 and PI filters with the DAPI and Qdot565 filters, multiple images of the same field of view within each well were taken. All of the wells were imaged with the Alexa Fluor 488, PI, DAPI, Qdot565 and TIC filters to assess cell viability, location, and structure. Well sub-sets a, b, c3.5-8, which contained the QD construct test solutions, were imaged with the application of the Qdot565 filter as a method for accurately distinguishing the QD constructs form the cells. Wells not containing QD constructs were imaged with the Qdot565 filter to assess how non-QD construct entities would appear when the filter was applied. The excitation/emission wavelength ranges associated with the Hoechst stain and the QD 
constructs were very similar. This similarity introduced wavelength of the laser used for the excitation of the Hoechst and QD-constructs

\subsection{Results}

\subsubsection{QD Construct Introduction into Keratinocyte Cell Cultures}

\subsubsection{QD Construct Control and Test Solutions - RI}

Each of the steps in creating the final QD construct solution resulted in alterations of the samples CMW, intensity, FWHM, and scattering of the excitation source. The progression of these changes is documented in Table 4.4 and Figures 4.9, 4.10, and 4.11. Two different graphs were created to illustrate the different CMW and intensities related to each stage in the QD construct progression. The graph in Figure 4.9 gives the fluorescence spectrums of the CdSe core sample 42612wCMBO.2 as well as each of the CdSe core samples from which it was comprised. The general shapes of all of the samples are indicative of the typical spectrum for a white core sample;

one primary peak at $490 \pm 10 \mathrm{~nm}$ and a broad rolling peak between 550 and $650 \mathrm{~nm}$. Filtering of the initial R1 QD construct solution with a 0.45 micron filter resulted in a noticeable difference in the CMW, from 552nm to 539nm, of the QD construct solution. Changes in intensity were minimal, and changes in FWHM were non-existent. 
Table 4.4 Progression of Changes in Fluorescence Spectrum During Synthesis of QD Construct Solution 42612wEN.2a

\begin{tabular}{|l|c|c|c|c|}
\hline \multicolumn{1}{|c|}{ Sample } & $\begin{array}{c}\text { Primary } \\
\text { Peak CMW } \\
\text { (nm) }\end{array}$ & $\begin{array}{c}\text { Intensity } \\
\text { (counts) }\end{array}$ & FWHM (nm) & $\begin{array}{c}\text { Source } \\
\text { Scattering } \\
\text { (counts) }\end{array}$ \\
\hline 11211AR1.5 & 486 & 4200 & - & - \\
\hline 2912AC1.0 & 490 & 7700 & - & - \\
\hline 21412AC1.0 & 486 & 6000 & - & - \\
\hline 21712AC1.0 & 473 & 5500 & - & - \\
\hline 22912AC1.0 & 485 & 6400 & - & - \\
\hline 42612wCMBO.2 & 487 & 6600 & - & 1300 \\
\hline 42612wQD.2a & 550 & 3000 & 50 & 500 \\
\hline 42612wEN.2a - NF & 552 & 16500 & 44 & 1300 \\
\hline 42612wEN.2a - 0.45 $\mu \mathrm{m}$ filter & 539 & 14600 & 44 & $>70000$ \\
\hline PL/krt [10mg/mL] - $0.2 \mu \mathrm{m}$ filter & 524 & 2400 & - & $>70000$ \\
\hline PL/krt $[10 \mathrm{mg} / \mathrm{mL}]-0.45 \mu \mathrm{m}$ filter & 524 & 2400 & - & $>70000$ \\
\hline
\end{tabular}

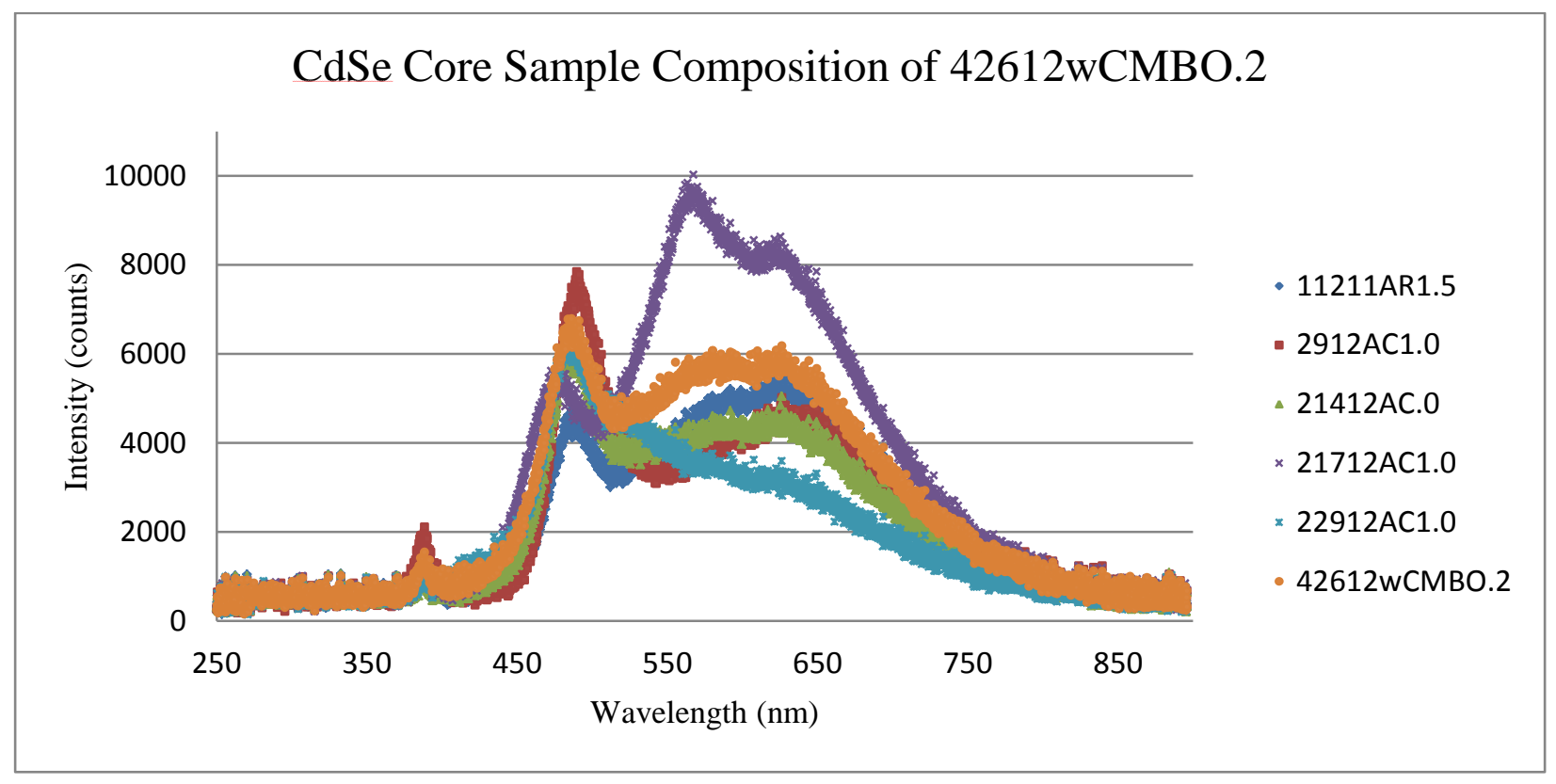

Figure 4.9 CdSe combination core sample (42612wCMBO.2 - orange) and the five CdSe core samples from which it was composed. 


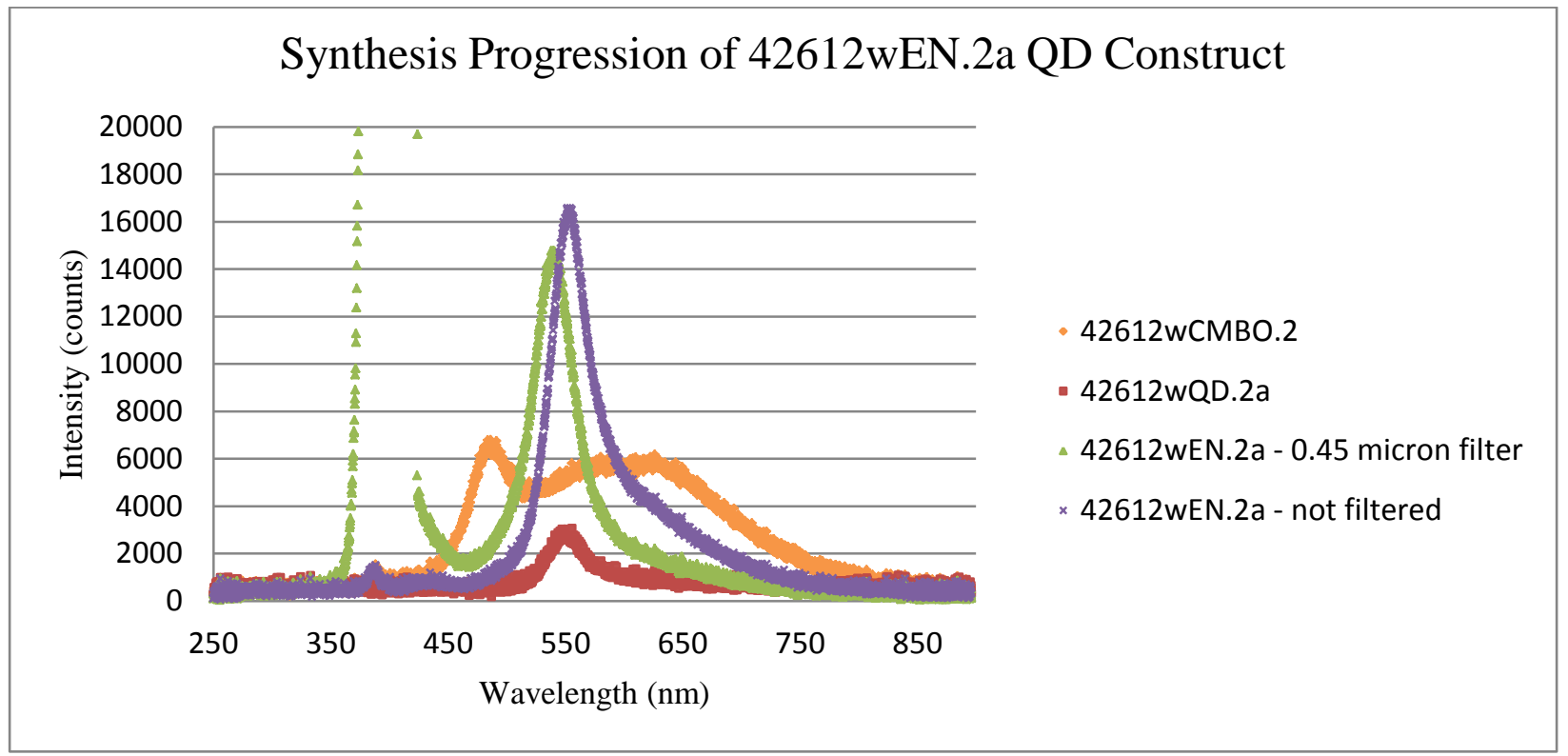

Figure 4.10 Changes in CMW, intensity, FWHM, and source scattering corresponding to solutions at each step in the QD construct synthesis process.

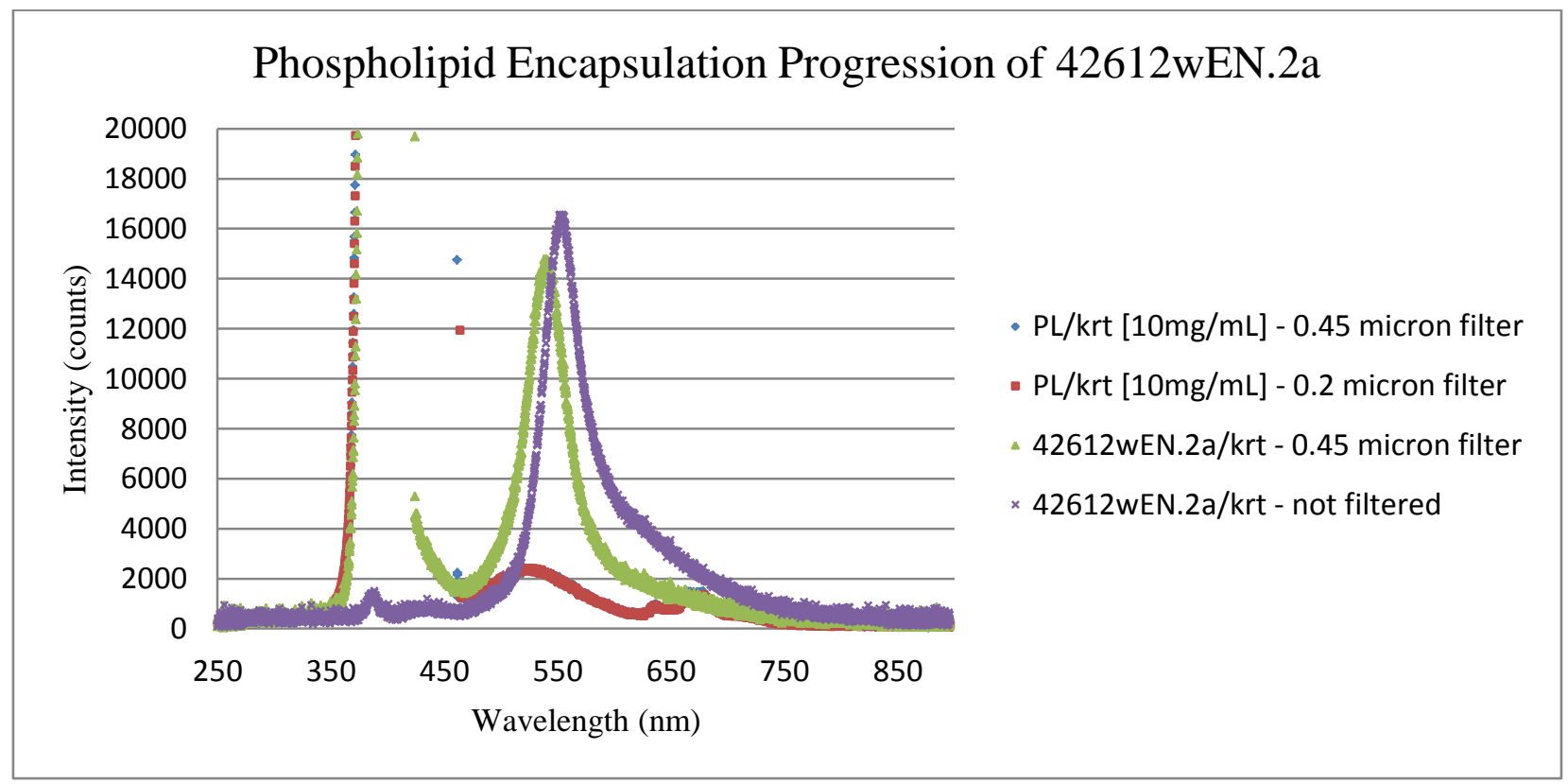

Figure 4.11Changes in CMW, intensity, FWHM, and source scattering corresponding to the influence of phospholipid concentration.

A useful option for qualitative assessment of the changes in CMW and intensity of each solution in the progression of QD construct development was found to be visual assessment of the samples 
during exposure to a UV source. Figure 4.12 provides a visual representation of each of the samples fluorescing.

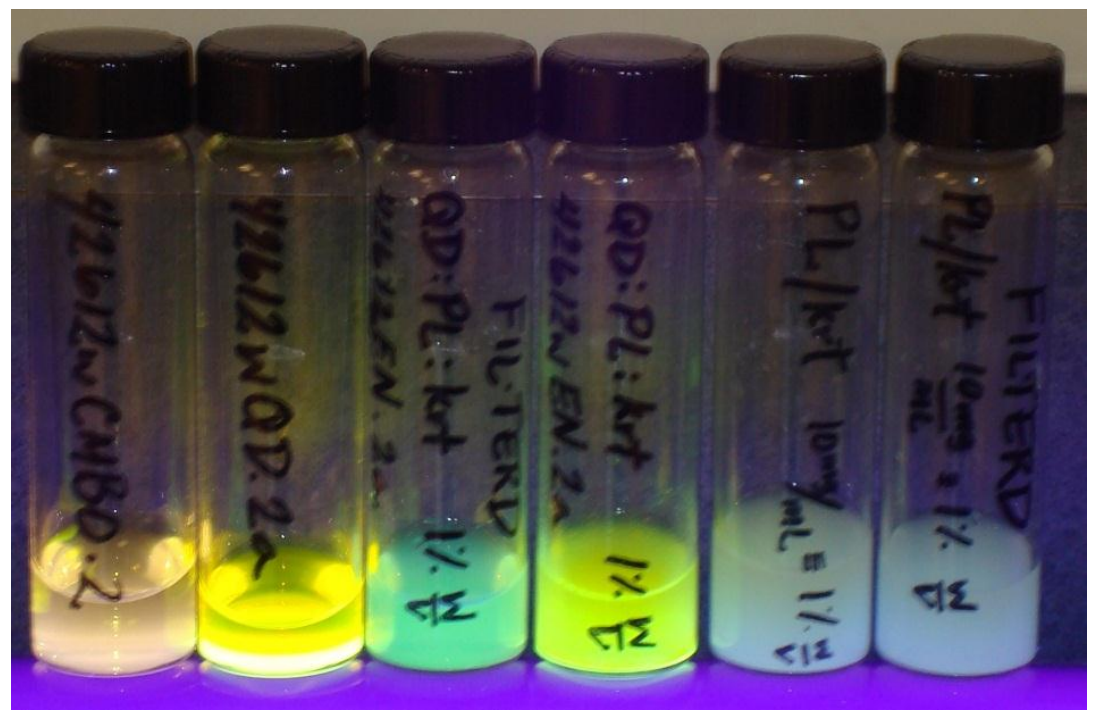

Figure 4.12 Progression of QD construct samples during exposure to a 400nm emitting black light. From left to right: Original CdSe core combination sample; CdSe/ZnS QD sample shelled at $160^{\circ} \mathrm{C}$; Phospholipid encapsulated QD construct solution after application of $0.45 \mu \mathrm{m}$ filter; Phospholipid encapsulated QD construct solution NOT filtered; PL/krt [10mg/mL] NOT filtered; $\mathrm{PL} / \mathrm{krt}[10 \mathrm{mg} / \mathrm{mL}]$ with application of $0.45 \mu \mathrm{m}$ filter.

\subsubsection{QD Construct Control and Test Solutions $-R 2$}

Although more data samples would be needed to definitively determine the effects of increased filtering on CMW, intensity, and FWHM of the QD constructs, quantitatively the effects appeared to be insignificant. Qualitatively, however, a visual comparison of the filtered samples showed a slight difference in the intensities of the two samples, as well as a noticeable shift in wavelength. It appeared as though the sample filtered through the 0.2 micron filter had a decreased wavelength relative to the sample filtered through a 0.45 micron filter. 


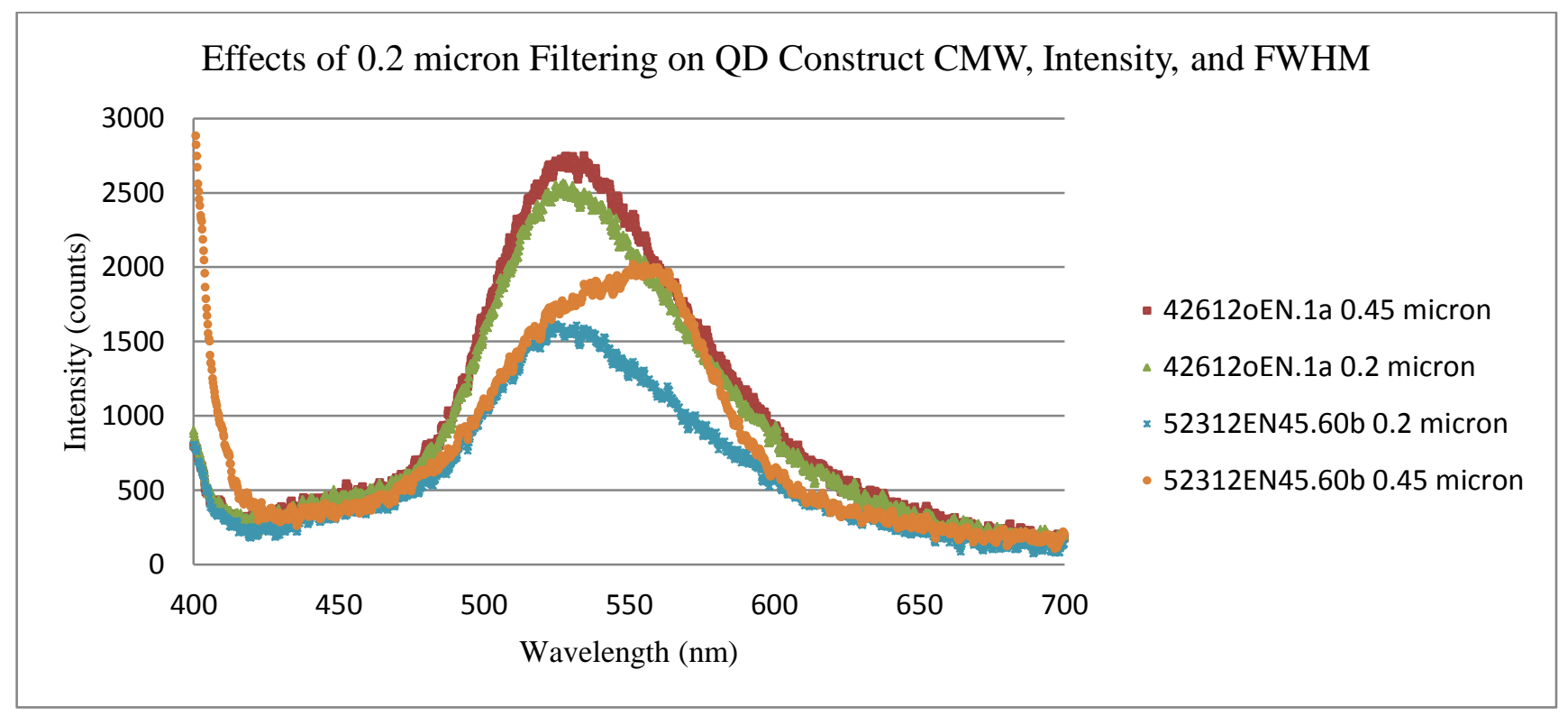

Figure 4.13 Fluorescence spectra of QD construct test solutions 42612oEN.1a and $52312 \mathrm{EN} 45.60 \mathrm{~b}$ filtered with 0.45 and 0.2 micron filters.

\subsubsection{Optical Imaging - RI}

The initial images of both well-sets of cell cultures, pre-introduction of the test solutions, showed a noticeable degree of contamination in each culture. Additionally, a noticeable amount of cell-like structures appeared at a higher focal plane and with a different morphology than the cells adhered to the surface of the well. Upon shaking of the four-well slide the cell-like structures jiggled but maintained their general position in the well. Had the cell-like structures fully detached from the surface of the well and freely moved in the upper portion of the media during agitation, the condition would have typically indicated that the cells were dead (89). The series of images in Figure 4.14 a representative example of the keratinocyte cell cultures as they appeared after being removed from the primary incubator before the aspiration of their initial media and introduction of the test solutions. In both sets of images contamination already appears to be present. 


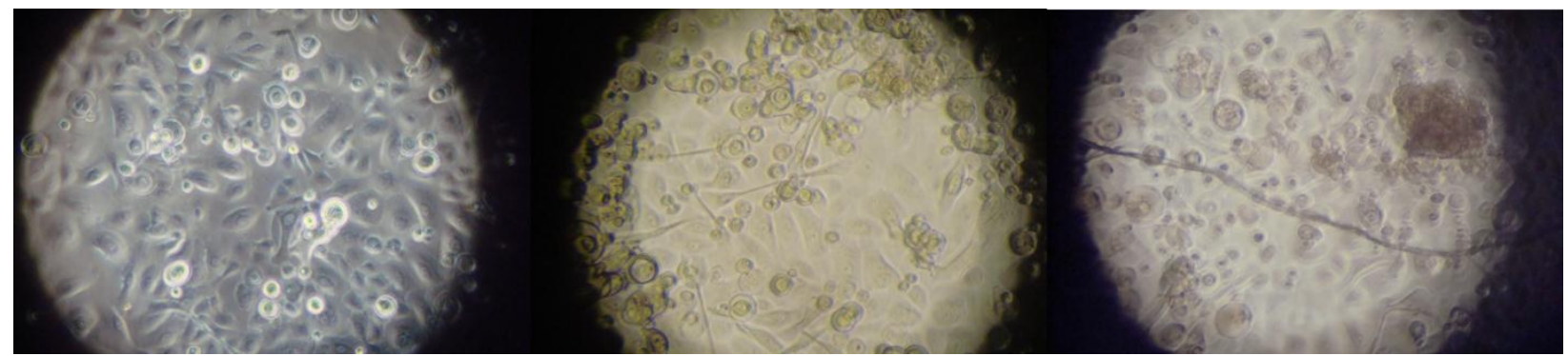

Figure 4.14 Digital white light optical images of R1a1.1, fresh media control, taken at different light intensities of the well pre-introduction of test solutions while the initial media was still present.

At the 24 hour time point post-introduction of the test solutions, the test solutions in the wells of well-set $\mathbf{b}$ were aspirated, rinsed with keratinocyte media, and imaged. All of the images show unidentified black orbs of unknown origin. A comparison of well b1.3 before and after the introduction of the test solutions is given in Figure 4.15. Images A1-3 are of the well b1.3, into which the test solution of keratinocyte media that had undergone the process involving exposure to both $\mathrm{CHCl}_{3}$, heating to $55^{\circ} \mathrm{C}$, and evaporation, would later be introduced. Images $\mathrm{B} 1-3$ are of this same well, b1.3, after a 24 hour exposure to the test solution, aspiration of the test solution and addition of fresh media. 


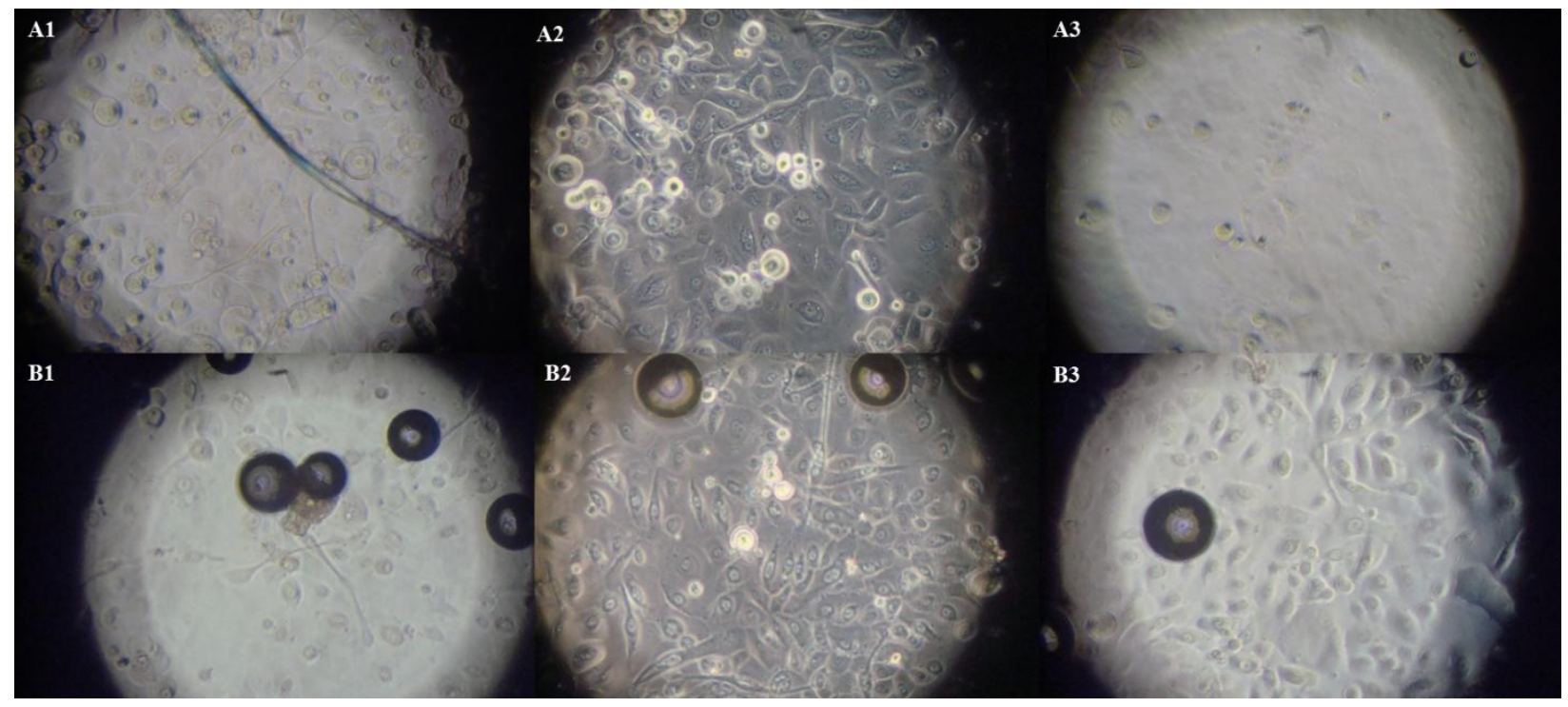

Figure 4.15 Digital white light optical images of R1b1.3 (keratinocyte media post $\mathrm{CHCl}_{3}$ evaporation control) taken at different light intensities. A1-3. Pre-introduction of test solutions while the initial media was still present. B1-3 Post-introduction of test solutions after aspiration and rinsing with fresh media

\subsubsection{Keratinocyte Cell Viability and Cell-QD Construct Interaction - R1}

The confocal equivalents of the white light optical images of well b1.3 are given in figure 4.16. Images $A 1,2$ and images B1, 2 each show two focal depths of a single field of view. In an attempt to discern the effect of the extended exposure of the cells to the L/D stain, a result of the time inherent to the process of imaging each well, a series of confocal images of well b1.1 is given in figure 4.17. These images taken are of cells that had only been exposed to the L/D stain for approximately 10 minutes. Each image is of the same well but at different fields of view and at sequentially lower focal planes. The order of the images is the order in which they were taken; approximately one minute passed in between capturing each image. As the images progress toward the right an increase in the number of cells fluorescing red, and therefore dead, can be detected. This increase in the appearance of dead cells is more influence by the change in focal plane than by the minute passage of time. The left most image is of the highest focal plane, the image farthest 
right is of the lowest focal plane; cells at the top of the culture versus cells closest to the surface of the slide, respectively.
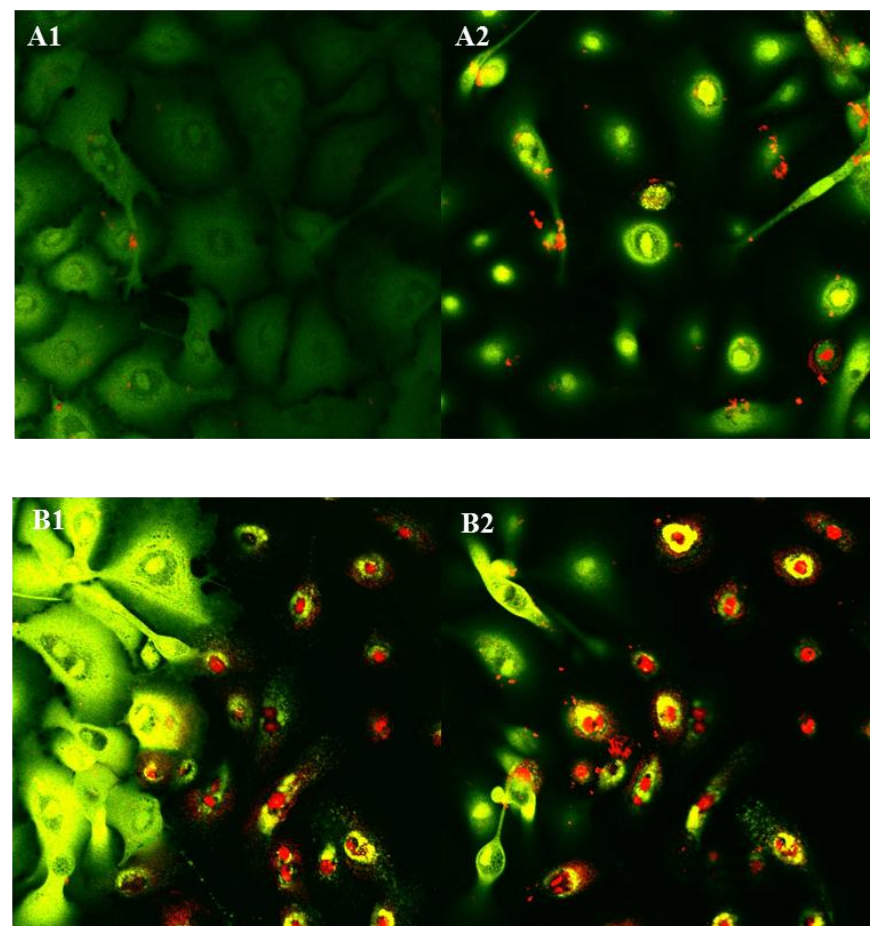

Figure 4.16 Confocal images acquired of well b1.3 (processed krt media control). A1. Two focal planes of one field of view. B1. Two focal planes of one field of view. Filters: Alexa Fluor 488 and PI

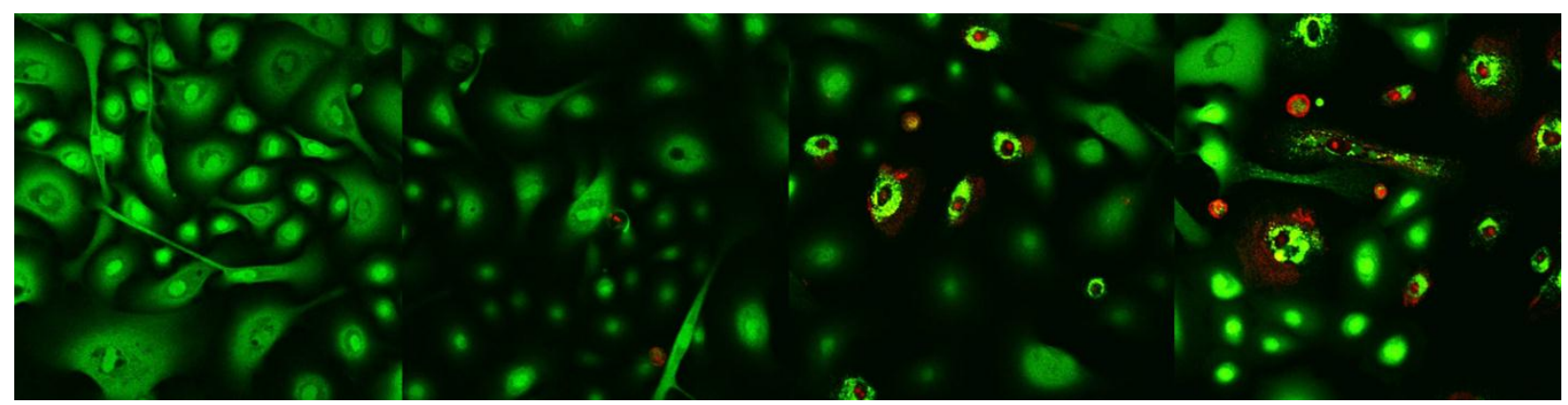

Figure 4.17 Series of confocal images of well b1.1 (fresh krt media control) at different fields of view and different focal planes. Filter: Alexa Fluor 488 and PI

Aside from the addition of the black orbs (bos), the average morphology and distribution of the cells in well b1.3 did not appear to be noticeably different from their appearance before the 
addition of the test solutions. The appearance of the orbs was consistent throughout all of well-set

b. The orbs appeared at greatest visual clarity at the same focal plane in which the cells appeared to be at the greatest visual clarity. The orbs were not distinguishable in the corresponding confocal images using the Alexa Fluor 488 and Pi filters shown in Figures

$4.18 \mathrm{C} 1-3$.
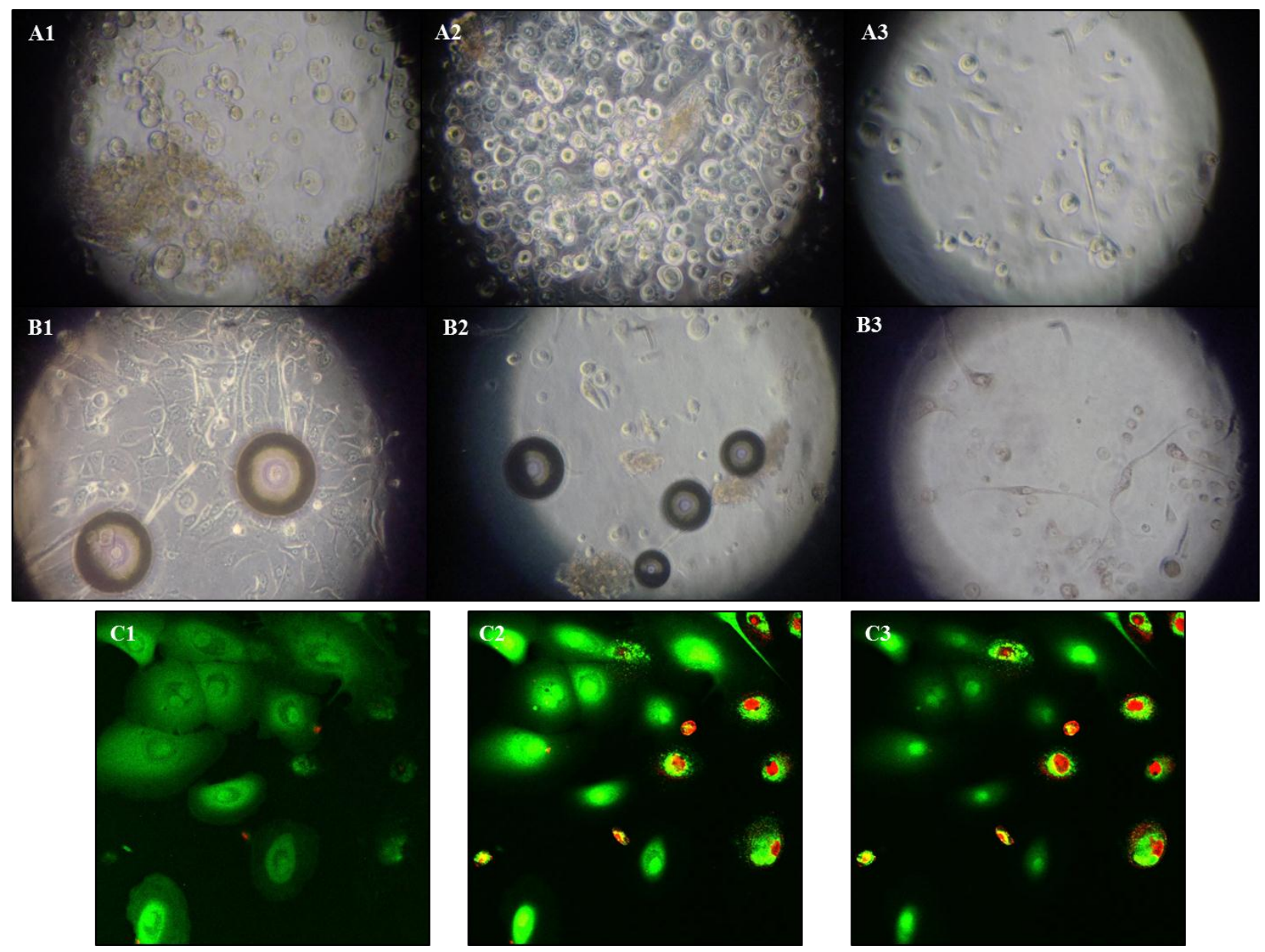

Figure 4.18 A1-3: Series of white light images of well b2.6 (PL krt filtered) pre-introduction of test solutions at different fields of view. B1-3 Series of white light images of well b2.6 postintroduction of test solutions at different fields of view. C1-3 Series of confocal images of well b2.6 in the same field of view at different focal planes. Filter: Alexa Fluor 488 and PI In both the white light optical images and confocal images of well b2.7 the presence of the phospholipids, filtered using a 0.45 micron filter, are clearly distinguishable. A span of images at three focal lengths was employed for the detection of dead cells fluorescing red. In the upper left 
corner of the confocal images in row $\mathrm{C}$, a structure unlike the cells is apparent. This structure could be representative of a black orb, made visible by the dense cell coverage.
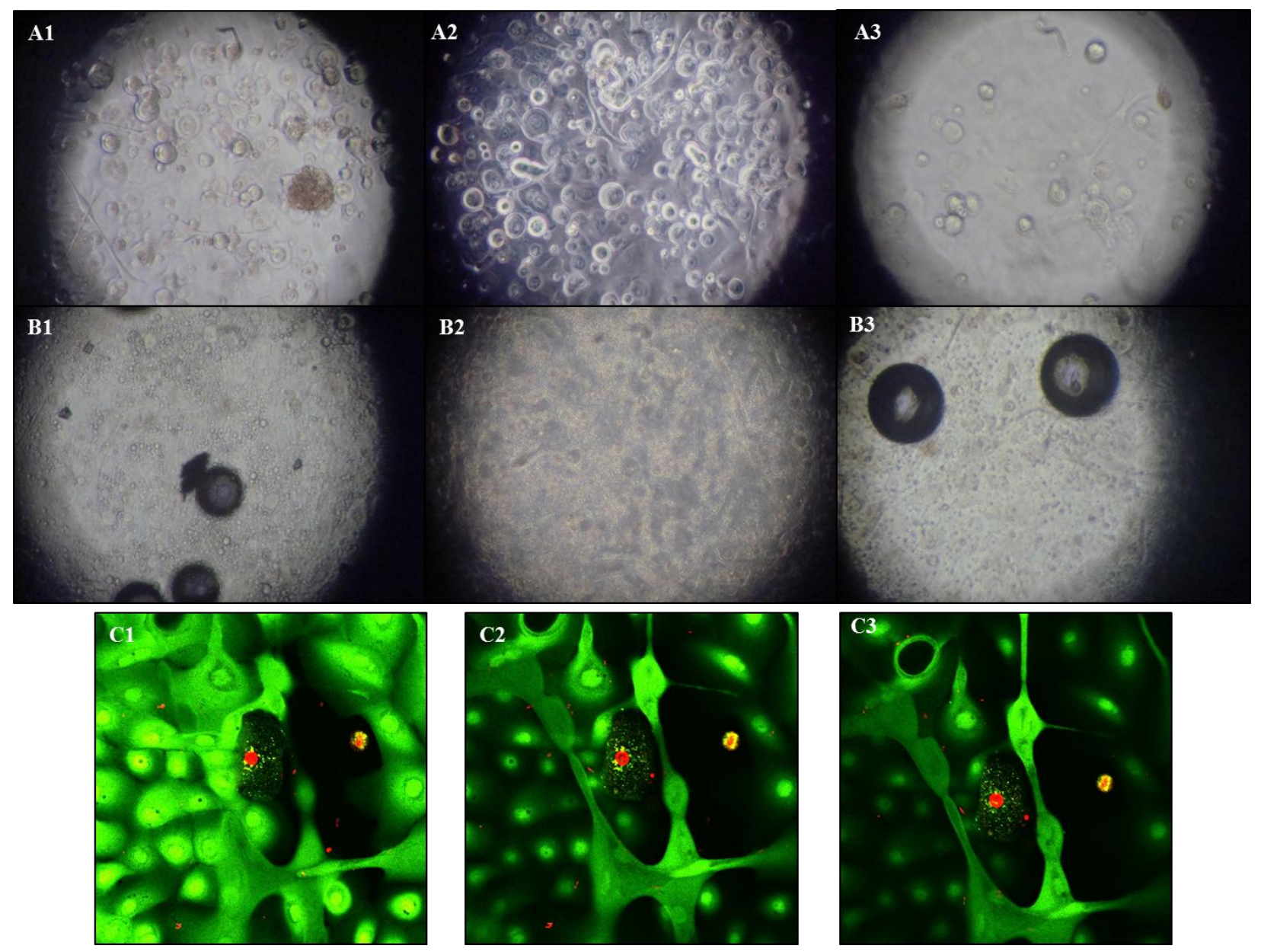

Figure 4.19 A1-3: Series of white light images of well b2.7 (PL krt NF) pre-introduction of test solutions at different fields of view. B1-3 Series of white light images of well b2.7 postintroduction of test solutions at different fields of view. C1-3 Series of confocal images of well b2.7 in the same field of view at different focal planes. Filter: Alexa Fluor 488 and PI.

Figure 4.20 gives a progression of images from the cultures exposed to the 0.45 micron filtered QD construct solution. Multiple black orbs were detected 24 hours after the introduction of the test solution. The structures identified as cells, those fluorescing both red and green with an obvious nucleus, show some evidence of interaction with the QD constructs. The wholly green spheres are 
may be the orbs and also show interaction with the QD constructs. The upper left sphere in C2 and the sphere in figure 4.20 each show a distinct QD halo indicating active interaction with the QDs.

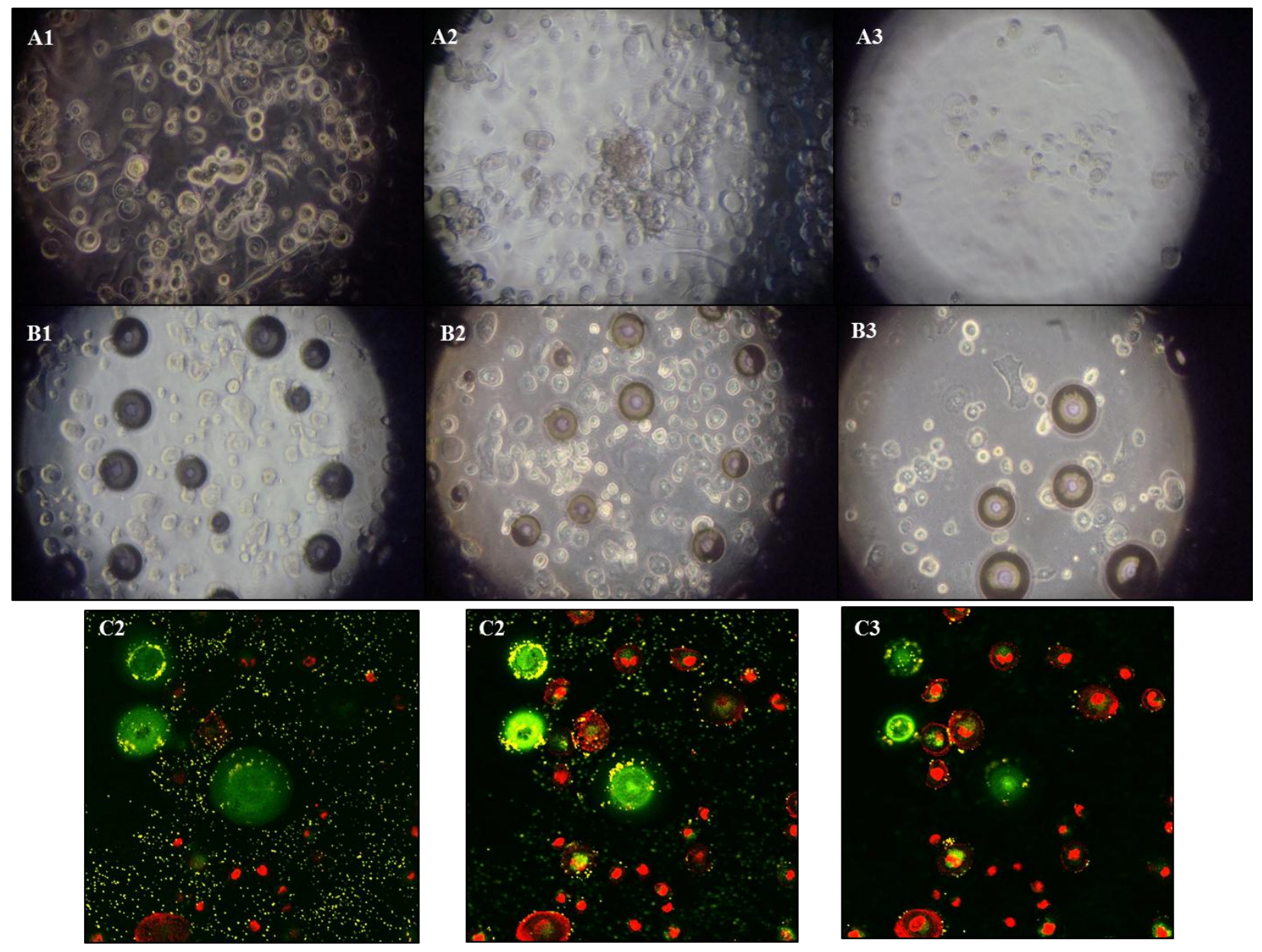

Figure 4.20 A1-3: Series of white light images of well b3.10 (QD PL krt filtered) pre-introduction of test solutions at different fields of view. B1-3 Series of white light images of well b3.10 postintroduction of test solutions at different fields of view. C1-3 Series of confocal images of well b3.10 in the same field of view at different focal planes. Filter: Alexa Fluor 488 and PI. 


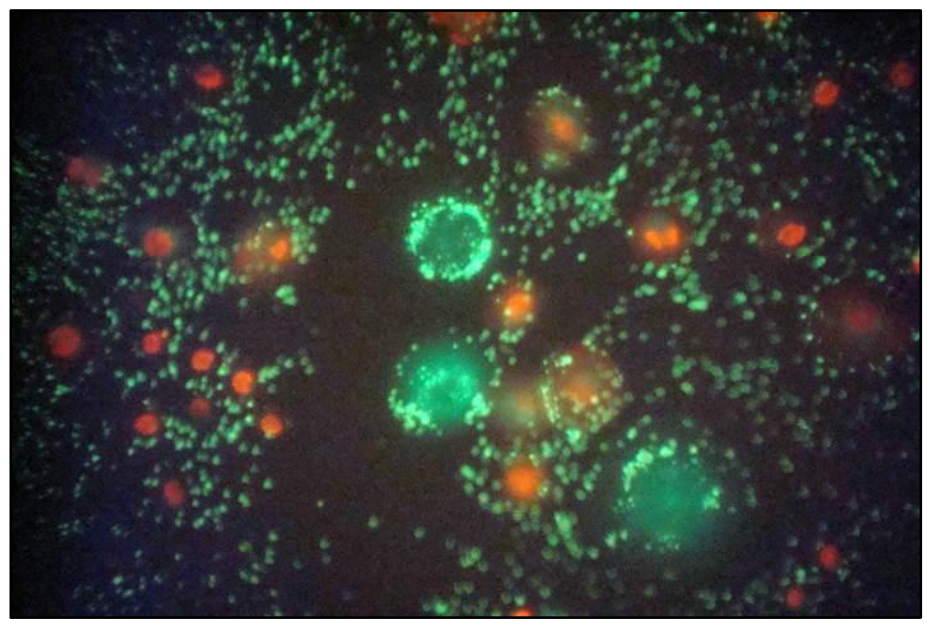

Figure 4.21 Digital image of well b3.10 as seen through the objective lens of the confocal microscope viewed with illumination from the wide-field fluorescence laser
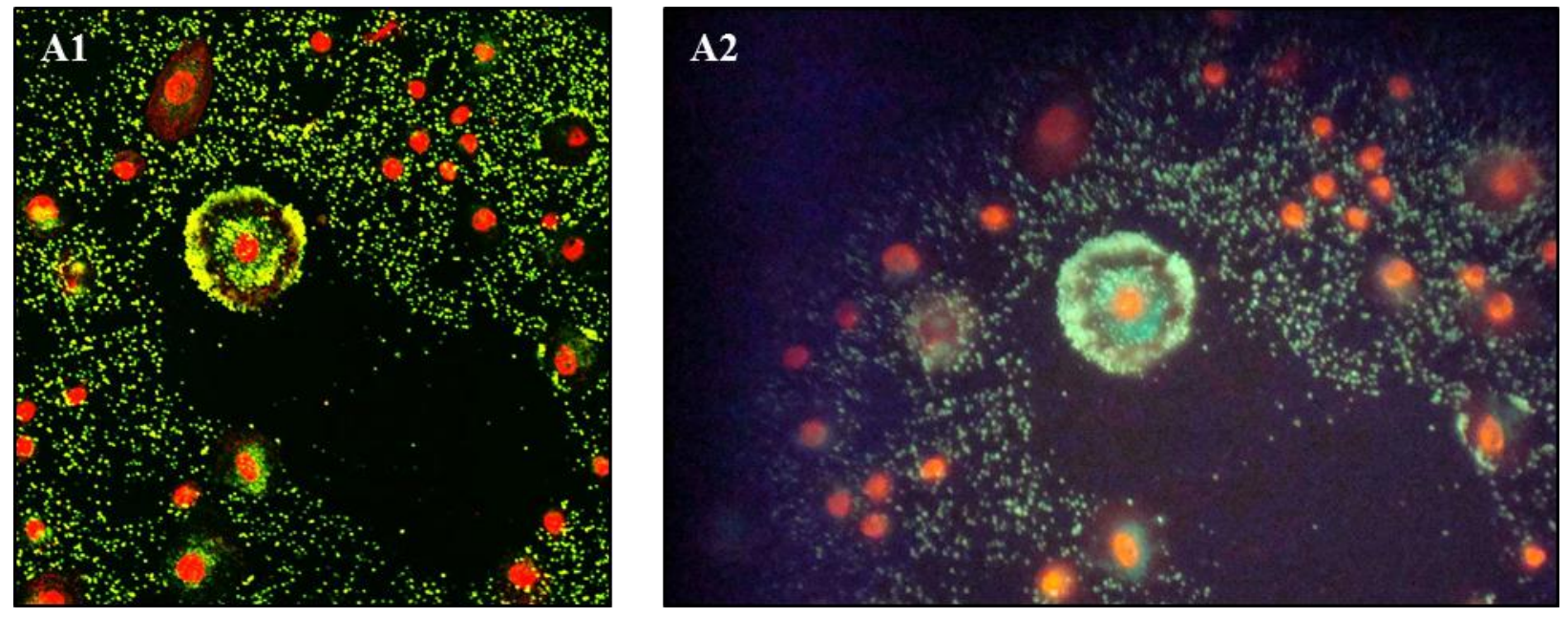

Figure 4.22 A1. Confocal image of a seemingly burst cell in well b3.10 (QD PL krt filtered) A2. Digital image of burst cell in b3.10 as seen through the objective lens of the confocal microscope viewed with illumination from the wide-field fluorescence laser. 

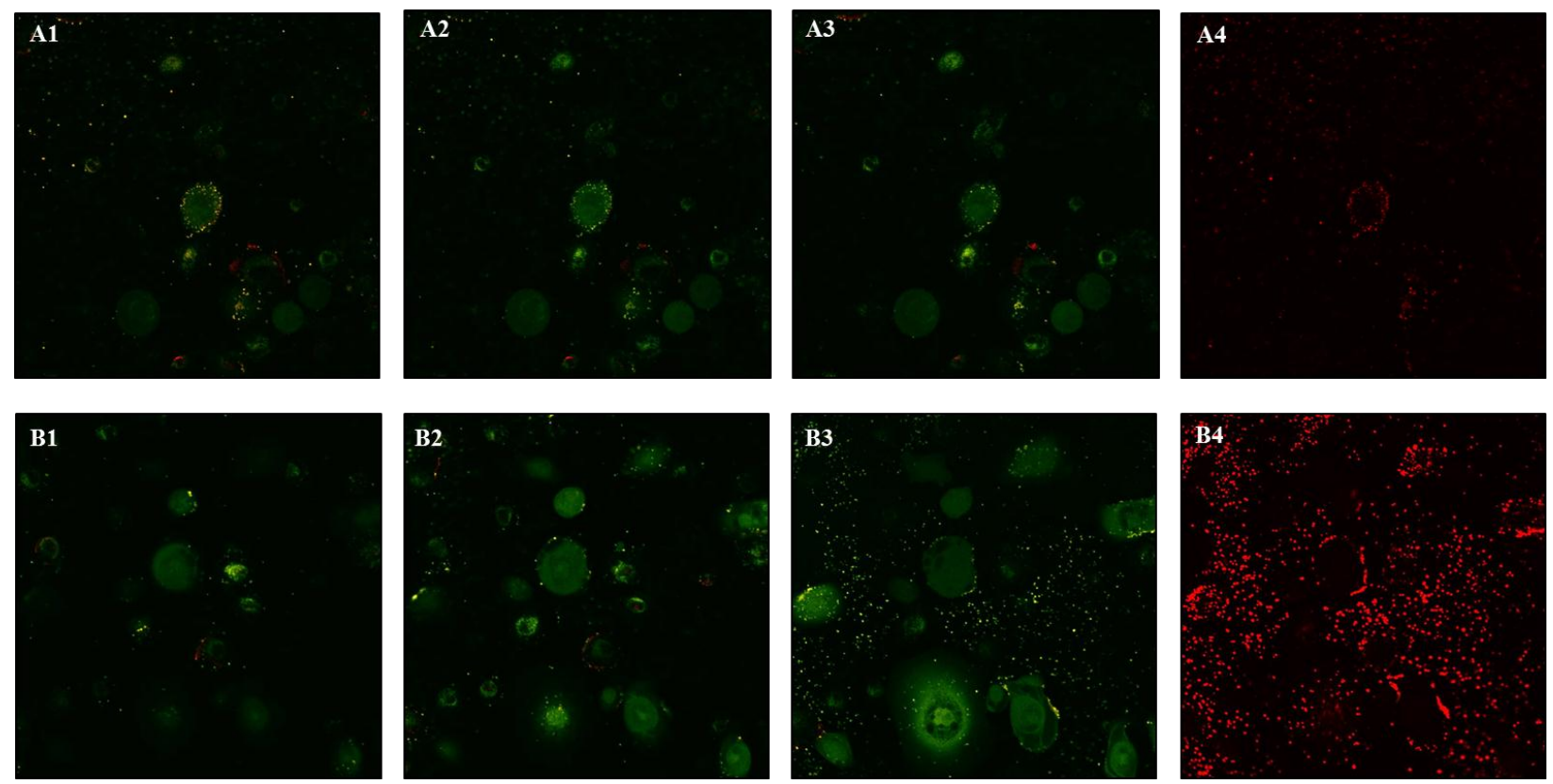

Figure 4.23 A1-3. Series of L/D filter confocal images of well a3.9 (QD PL krt filtered) A4 Qdot565 filter. B1-3. Series of L/D filter confocal images of well a3.9 (QD PL krt filtered) B4 Qdot565 filter.

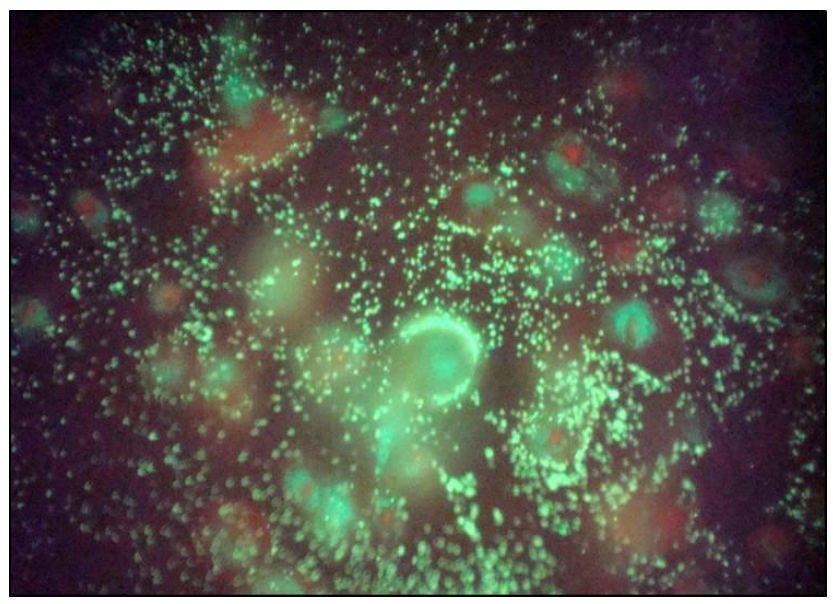

Figure 4.24 Digital image of well a3.9, representative of the series A1-4 in figure 4.23 as seen through the objective lens of the confocal microscope viewed with illumination from the wide-field fluorescence laser. 

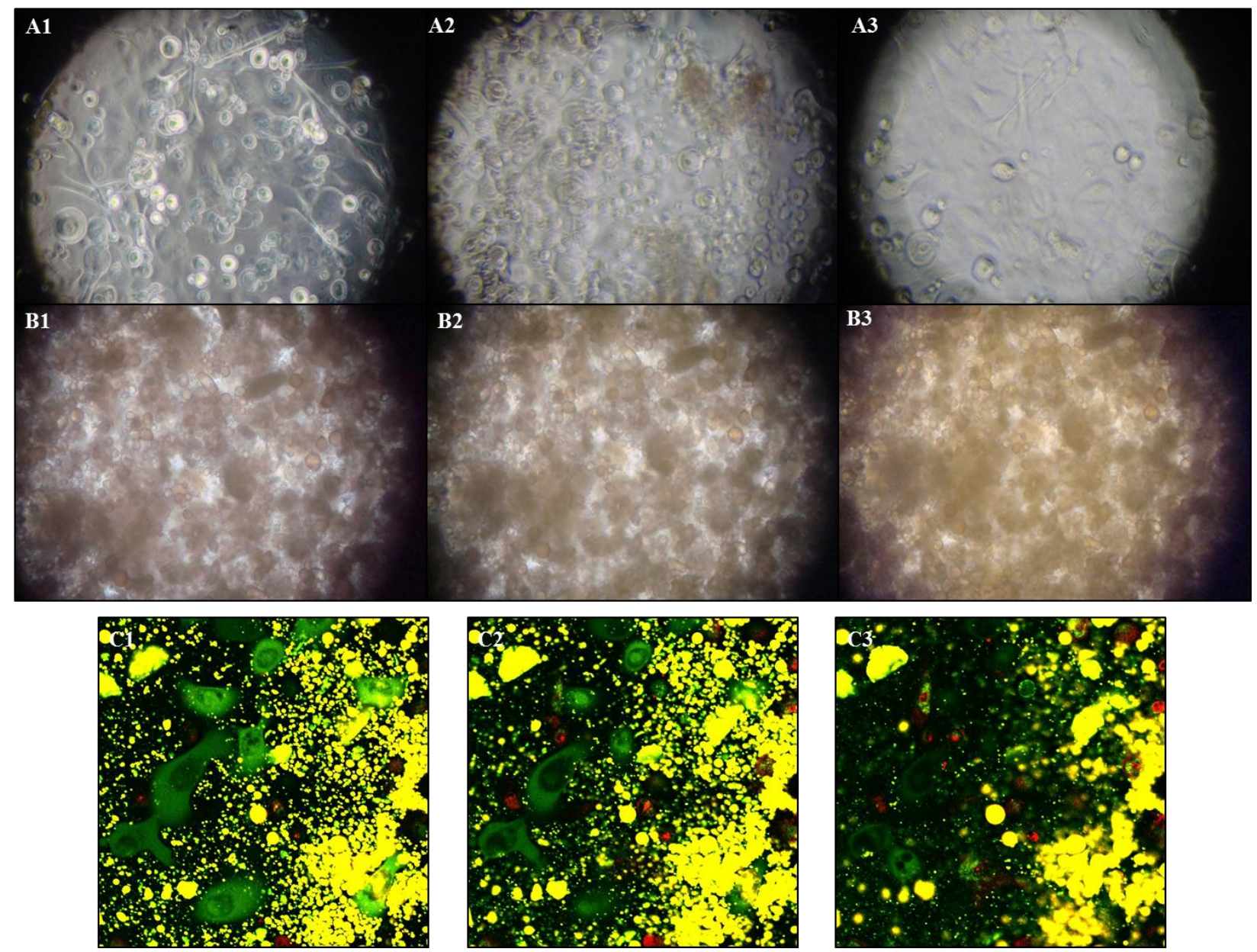

Figure 4.25 A1-3: Series of white light images of well b3.12 (QD PL krt NF) pre-introduction of test solutions at different fields of view. B1-3 Series of white light images of well b3.12 postintroduction of test solutions at different fields of view. C1-3 Series of confocal images of well b3.12 in the same field of view at different focal planes. Filter: Alexa Fluor 488 and PI 

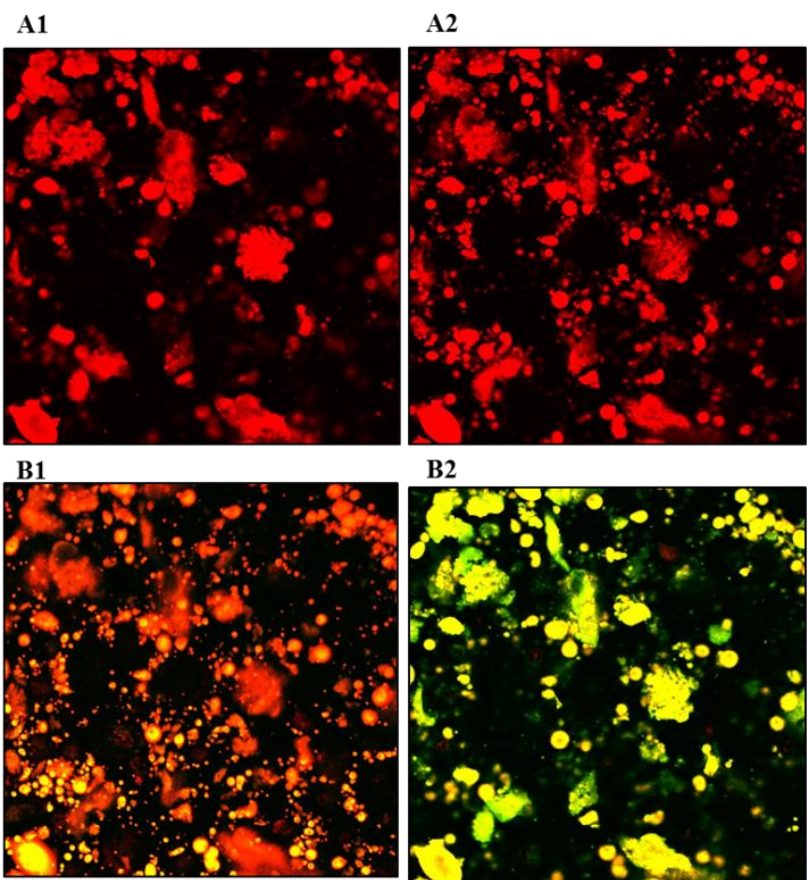

A2

A3
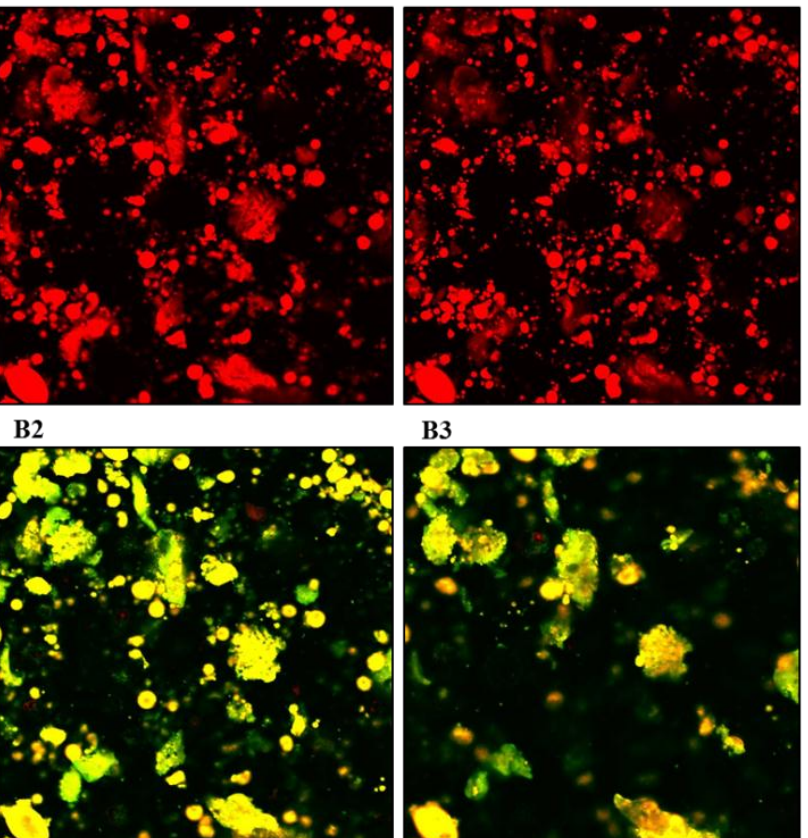

B3

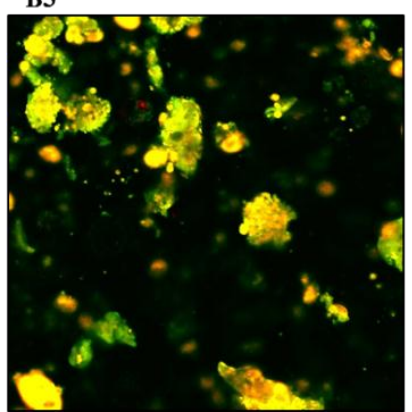

A4

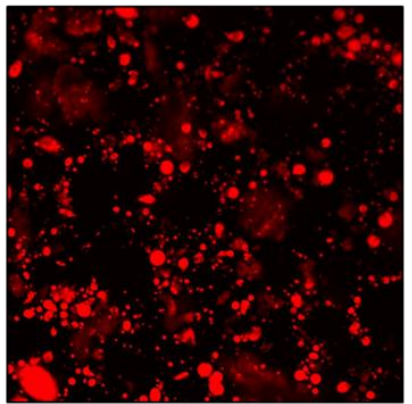

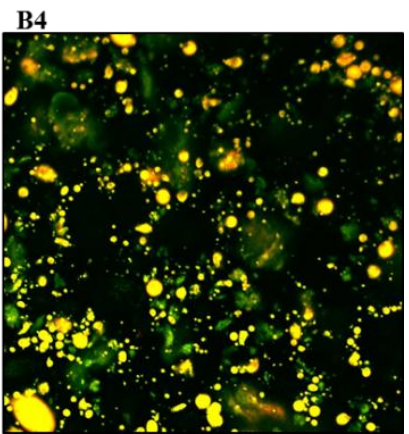

Figure 4.26 Series of confocal images of well a3.11 (QD PL krt NF) at the same field over a series of focal planes. Filters: A1-4 Qdot565, B1-4 Alexa Fluor 488 and PI

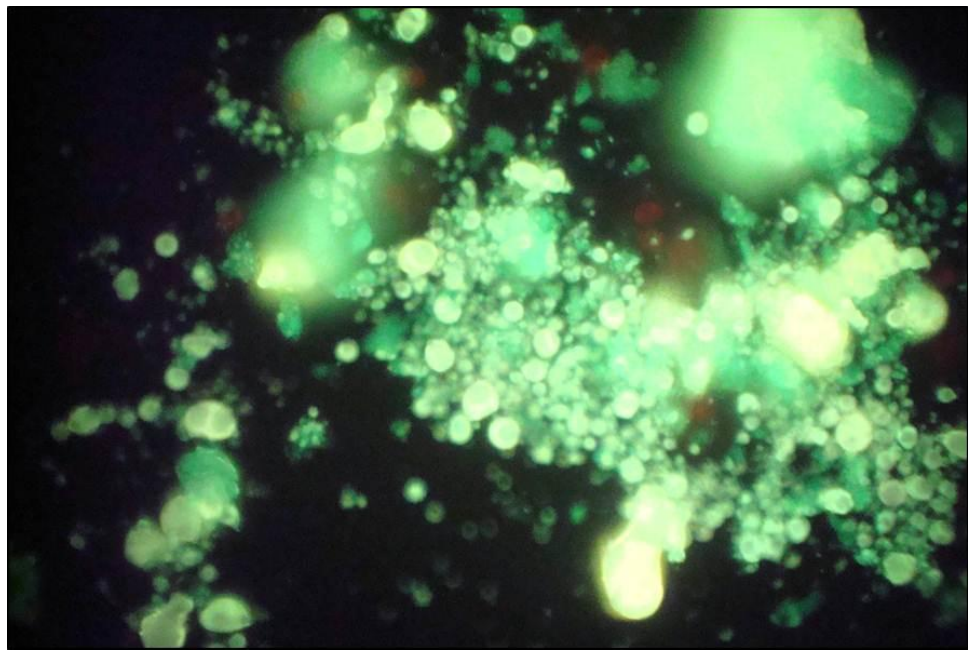

Figure 4.27 Digital image of a3.12 (QD PL krt NF) as seen through the objective lens of the confocal microscope viewed with illumination from the wide-field fluorescence laser.

\subsubsection{Imaging $-R 2$}

All of the initial cell cultures showed similar levels of minimal contamination and cell confluency, approximately $60 \%$, at the time of extraction form the primary incubator and before 
the introduction of the test solutions. Figure 4.26 gives a sampling of the culture appearance from each well-set pre-introduction of the test solutions. At one hour post-introduction of the test solutions well-set a showed, on average, two of the spherical unknowns for every three wells. There was no apparent correlation between the test solution administered to the well and the appearance of the bos. Wells that had contained controls of fresh keratinocyte media, phospholipids in keratinocyte media, QD constructs, and both levels of filtration all contained at least one black orb when all of the related wells were considered as a group. Figure 4.27 gives a sampling of the culture appearance from each well-set post-introduction of the test solutions. The sample includes images representing each of the three time points along with each of the test solutions. 

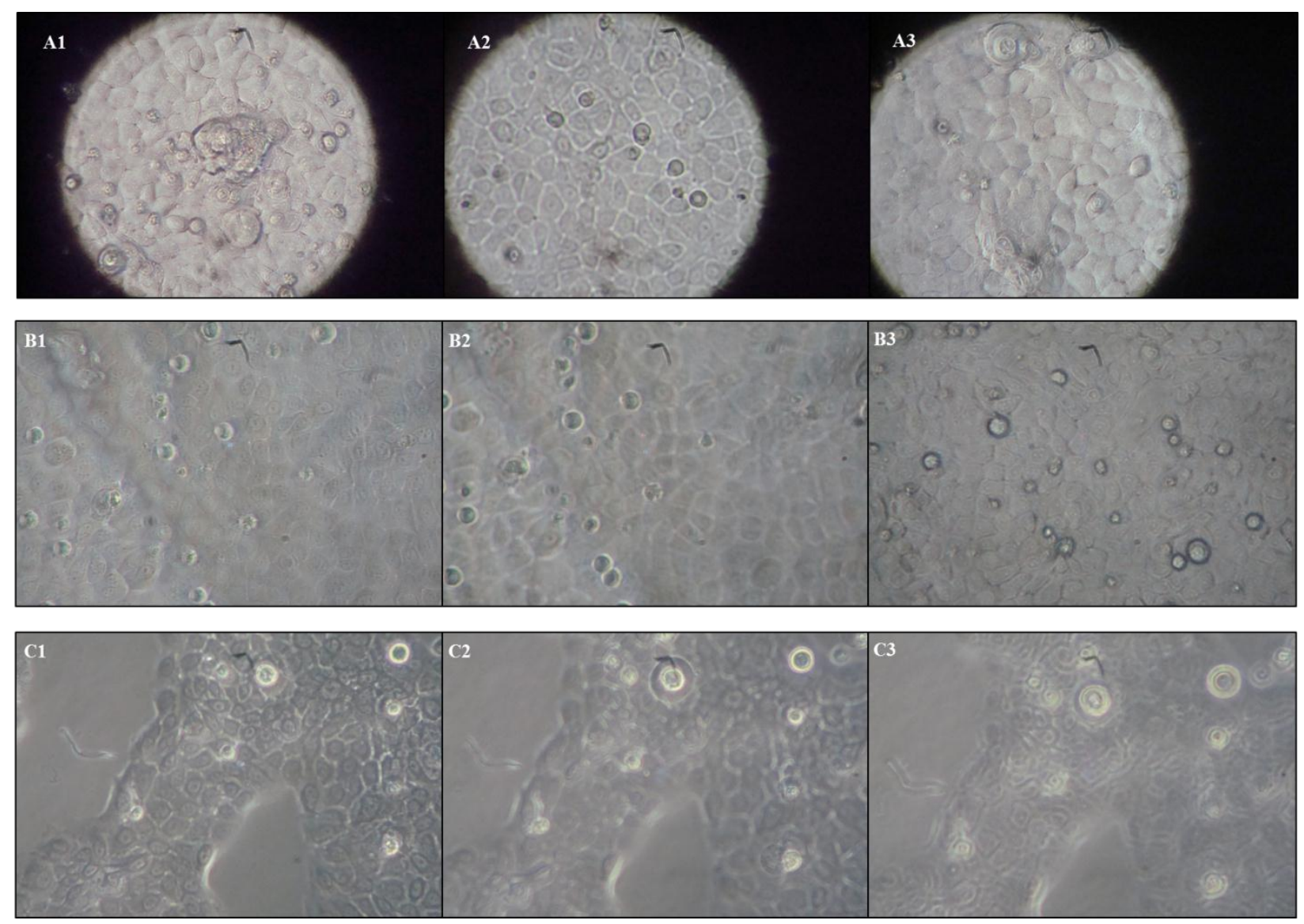

Figure 4.28 Representative images taken with a white light optical microscope pre-introduction of the test solutions. A. well-set a. B. well-set b. C. well-set c, a single field of view at three different focal planes to better illustrate the culture composition. 

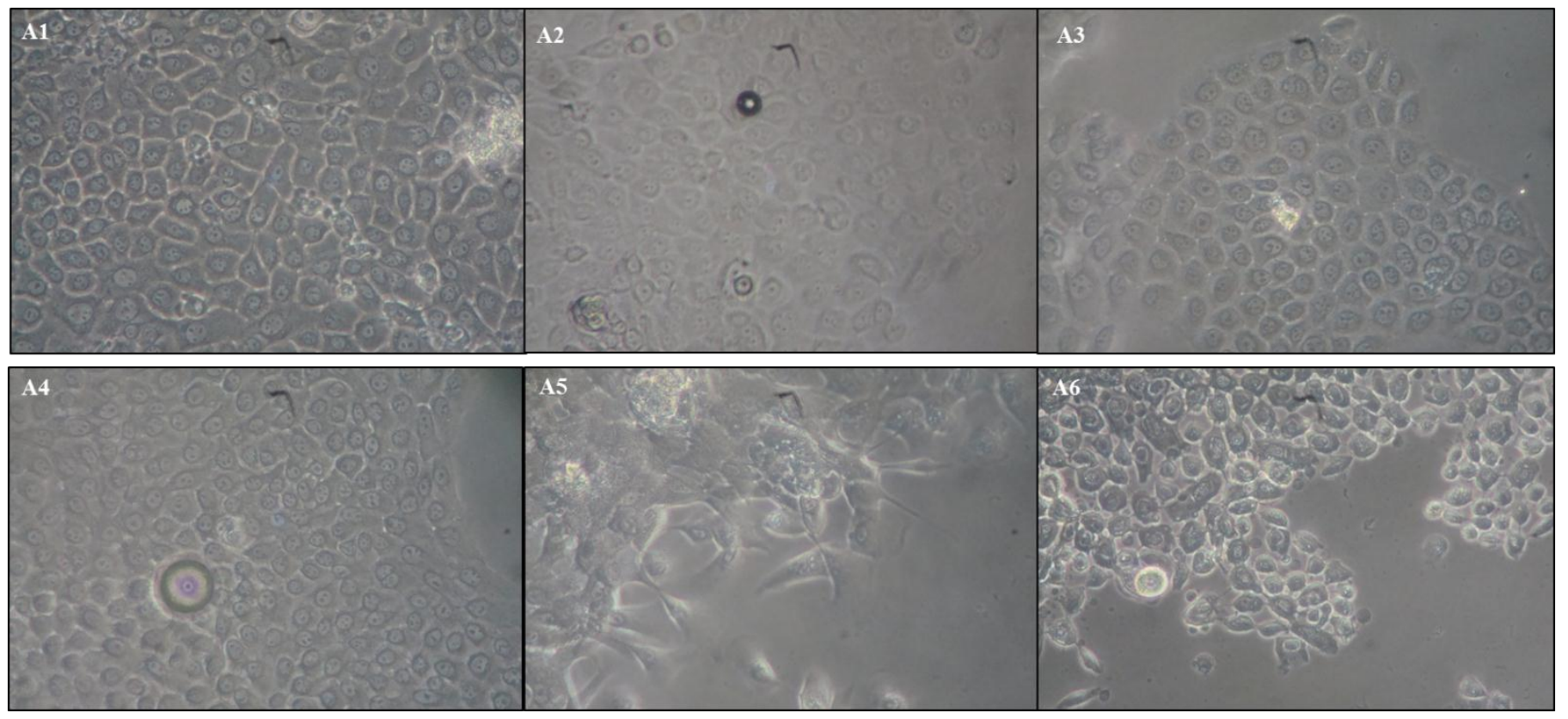

Figure 4.29 Representative images taken with a white light optical microscope post-introduction of the test solutions. A1,2. a1.4 QD PL B 0.2 micron filter. A3. a1.2 QD construct A 0.2 micron filter. A4. a2.8 PL 0.45 micron filter. A5. c1.3 PL 0.2 micron filter. A6. c1.4 QD construct B 0.2 micron filter.

\subsubsection{Keratinocyte Cell Viability and Cell-QD Construct Interaction - R2}

Confocal images of well sub-set a1.1-4 lacked clarity as a result of the first method applied in the arrangement of the cover-slip and specimen slide. The two images acquired at one hour post introduction of the test solutions are given in Figure 4.28. The images are of well a1.1, which contained the keratinocyte media control solution and was prepared with the L/D stain. An additional effect of the slide preparation issue was the delayed imaging of well sub-set a2.5-8. All images of well sub-set a2.5-8 were taken 24 hours after the test solutions had been removed; the wells were rinsed and subsequently left covered but un-incubated for 24 hours. As a result of the Hoechst stain being unavailable for use in well-set $\mathbf{a}$, there are no confocal images of well-set $\mathbf{a}$ with the DAPI filter applied; all images are of cultures exposed to the L/D stain and the imaged using the Alexa Fluor 488, PI, Qdot565, and TDI filters. 

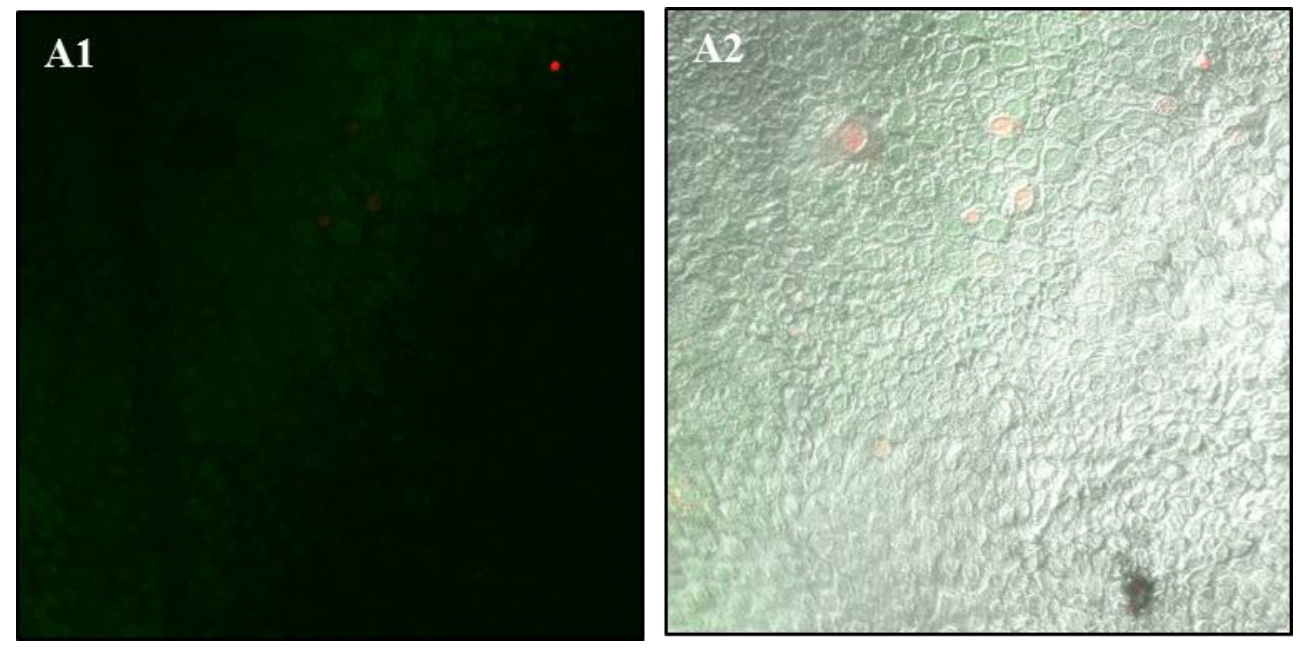

Figure 4.30 Well a1.1 (keratinocyte media control). A1. Alexa Fluor 488 and PI filter. A2. Alexa Fluor 488, PI, and TDI filter overlay.

The confocal images taken of the wells exposed to the QD construct test solutions showed a minimal presence of QD constructs in the culture. The yellow arrow in Figure 4.29 indicates the single QD construct that was visible in that field of view of well a2.5. An additional series of images using each filter was applied to well b1.4, also exposed to a QD construct test solution. 

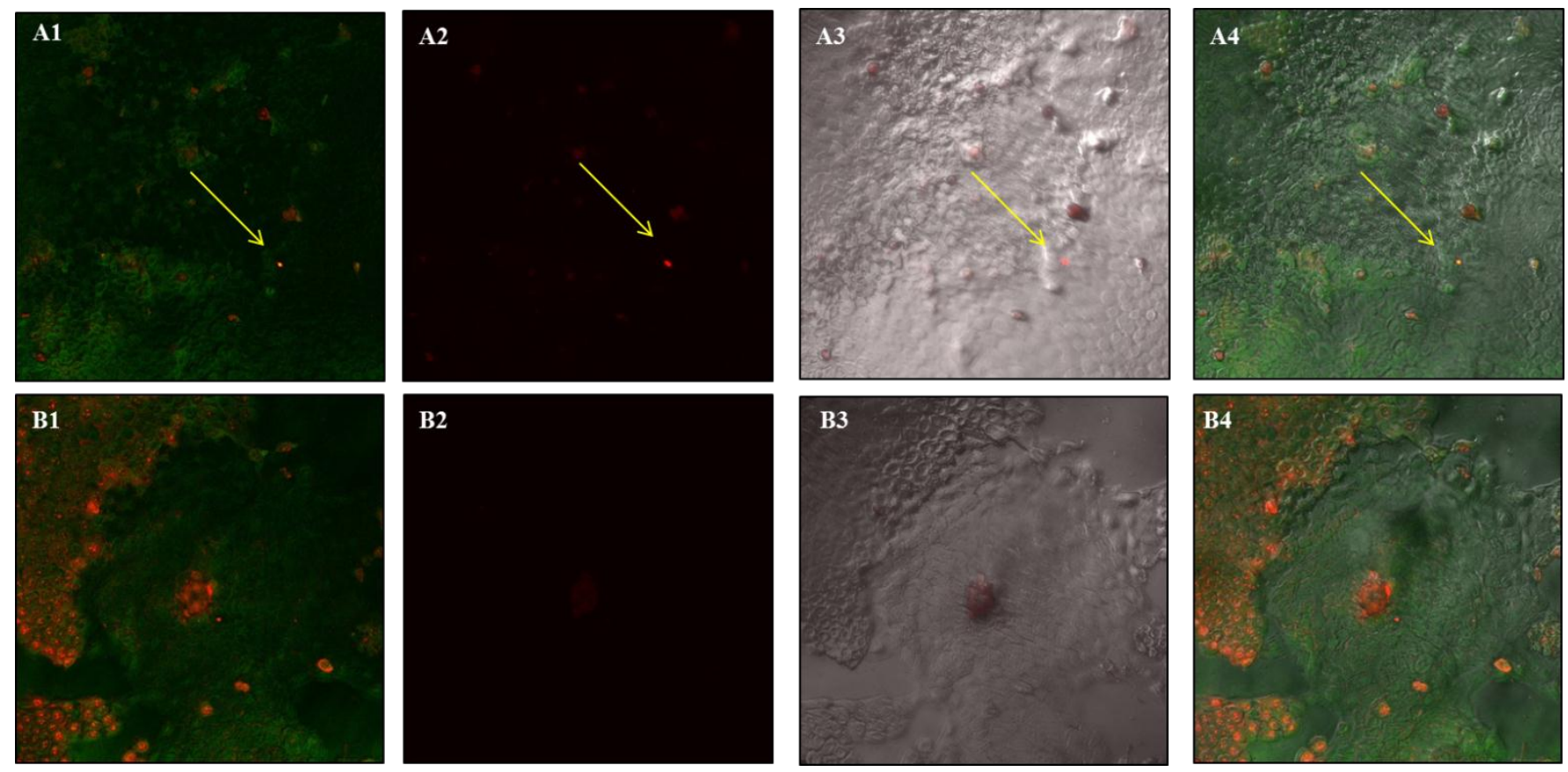

Figure 4.31 Comparison of well a2.5 (QD construct A 0.45 micron filter) in row A with well a2.6 (keratinocyte media control) in row B. Column 1 - Alexa Fluor 488 and PI filter. Column 2 Qdot565 filter. Column 3 - Qdot565 overlaid with TDI. Column 4 - Alexa Fluor 488 and PI with TDI filter overlay. The yellow arrow points to a QD construct.
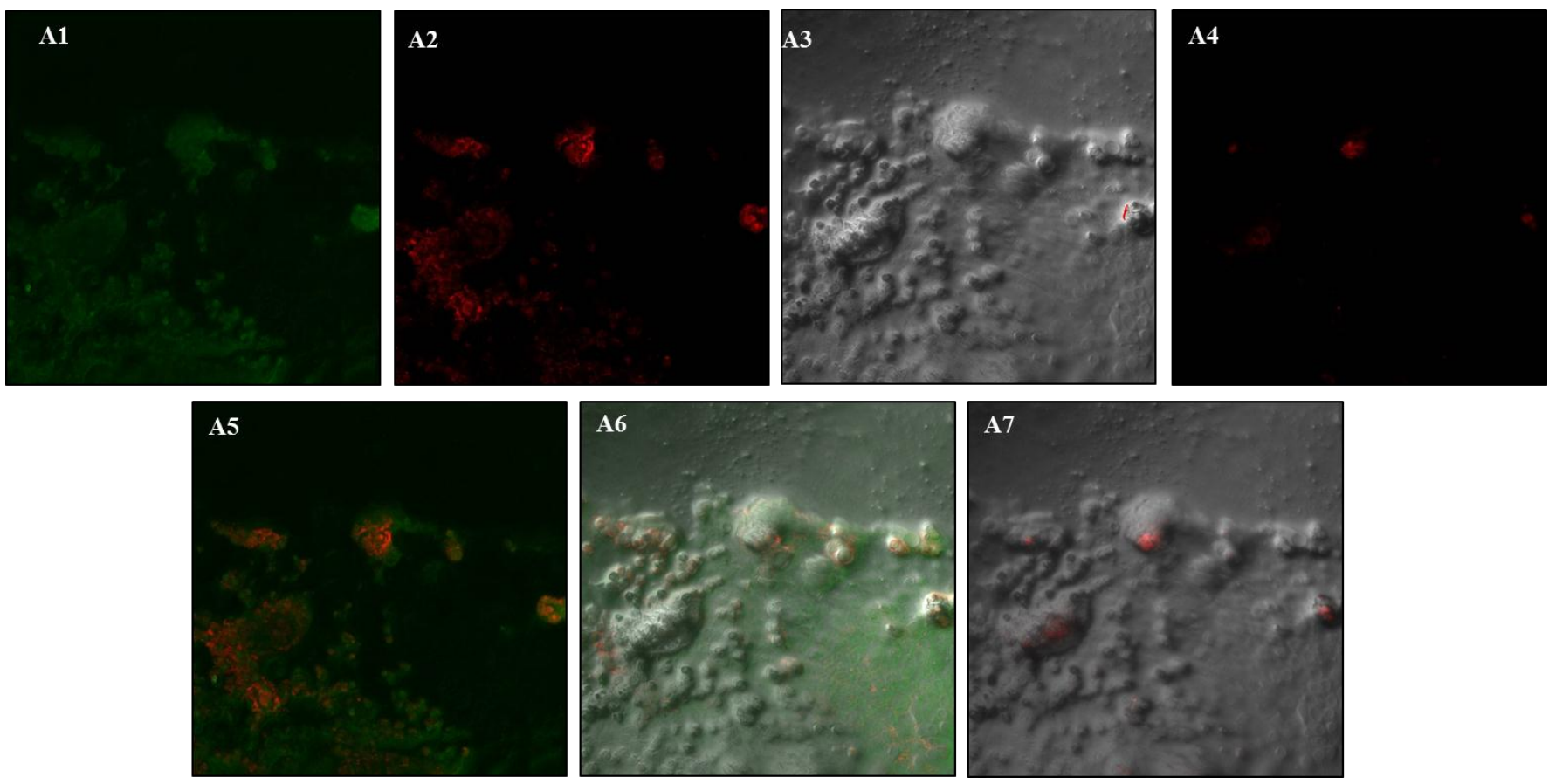

Figure 4.32 Series of confocal images taken of b1.4 (QD construct B 0.2 micron filter). Filters applied: A1- Alexa Fluor 488; A2 - PI; A3 - TDI; A4 - Qdot565; A5 - Alexa Fluor 488 and PI; A6 - Alexa Fluor 488, PI, and TDI; A7 - Qdot565 and TDI. 
The series of images in Figures 4.33 and 4.34 both indicate the identification of QD

constructs. Well b2.5 of figure 4.33 did contain cells exposed to QD constructs. Well b2.6 in Figure 4.34 did not contain QD constructs. Well b2.6 contained only keratinocyte media. The

identification of QD contructs in this well is a false identification the result of the Hoechst stain being excited by the 405 excitation wavelength and the subsequent emission form the Hoechst stain being detected by the Qdot565 filter. Figures 4.35 and 4.36 give a side by side comparison, of the same field of view, for images resulting from use of the $405 \mathrm{~nm}$ excitation source versus that of the 488nm excitation source. These images illustrate the false identification of QD constructs when the 405nm laser is applied to wells with the Hoechst stain and detected with the Qdot565 filter. Additionally these images show that an excitation wavelength of $488 \mathrm{~nm}$ does not produce a false positive for the presence of QC constructs.
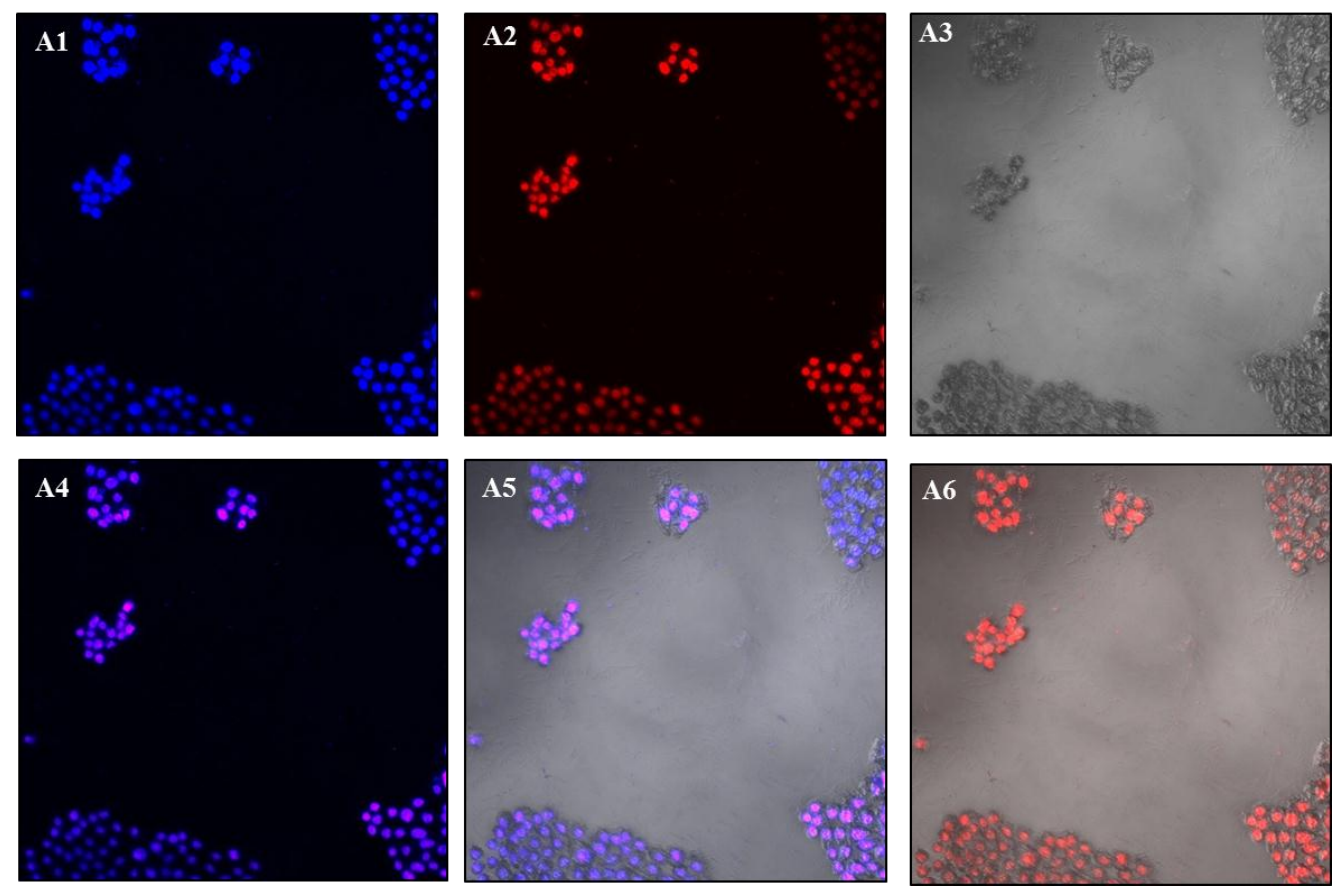

Figure 4.33 Series of confocal images taken of b2.5 (QD construct A 0.45 micron filter). Filters applied: A1- DAPI; A2 - Qdot565; A3 - TDI; A4 - DAPI and Qdot565; A5 - DAPI, Qdot565, and TDI; A6 - Qdot565 and TDI. 

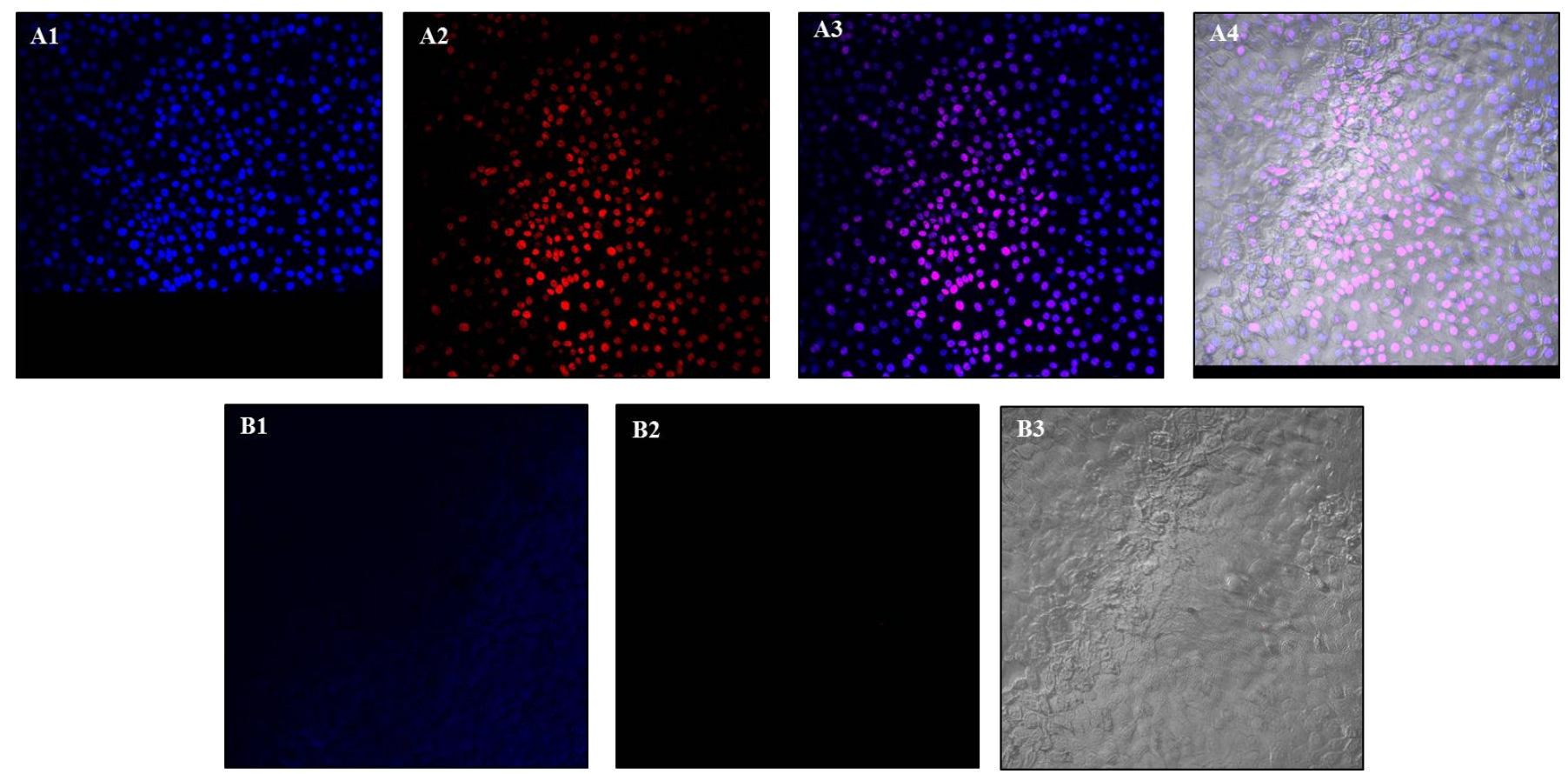

Figure 4.34 Series of confocal images taken of b2.6 (keratinocyte media control) comparing excitation wavelengths of 405 and $488 \mathrm{~nm}$. Row A utilizing 405nm and row B utilizing 488nm. Filters applied: A1- DAPI; A2 - Qdot565; A3 - DAPI and Qdot565; A4 - DAPI, Qdot565, and TDI; B1 - DAPI; B2 - Qdot565; B3 - Qdot565 and TDI.
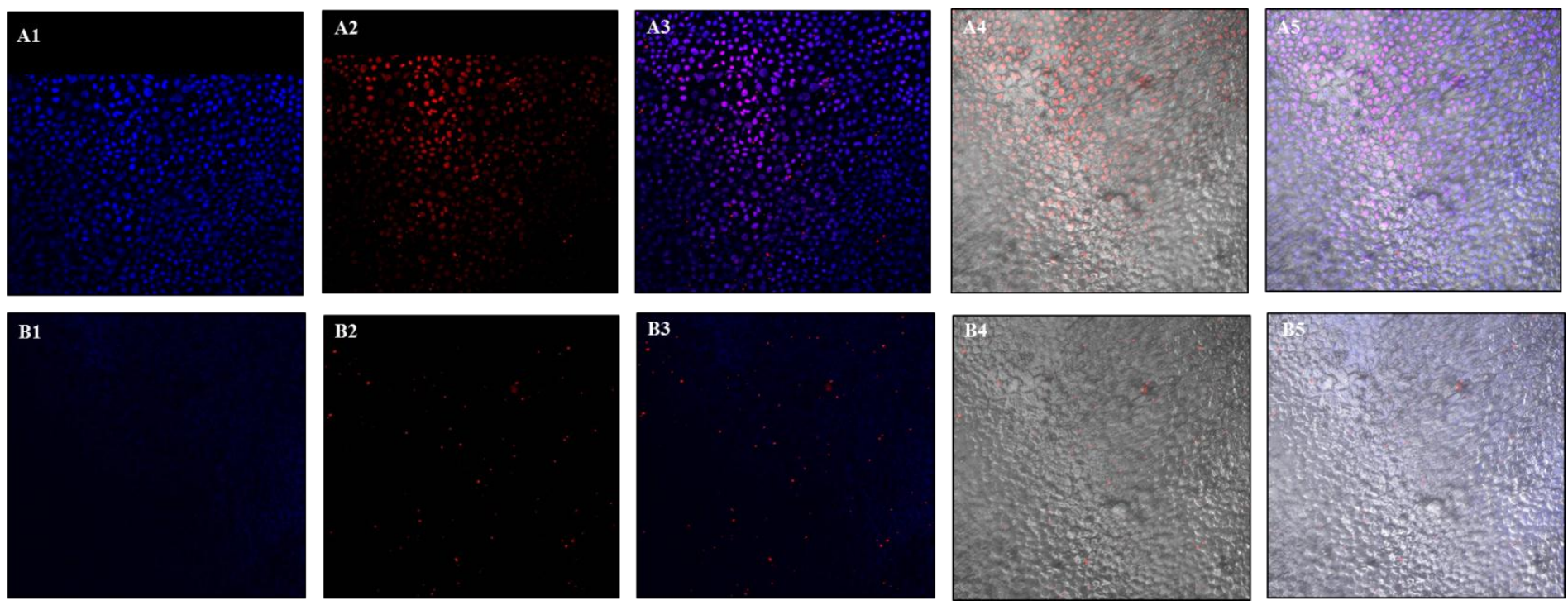

Figure 4.35 Series of confocal images taken of b2.7 (QD construct B 0.45 micron filter) comparing excitation wavelengths of 405 and 488nm. Row A utilizing 405nm and row B utilizing 488nm. Filters applied to each column: 1- DAPI; 2 - Qdot565; 3 - DAPI and Qdot565; 4 - Qdot565 and TDI; 5 - DAPI, Qdot565, and TDI. 
When comparison of the number of live to dead cells was made, comparing the effect of each test solution on cell viability, no significant difference was determined to be present. All wells, on average, had the same ratio of live to dead cells. Figures 4.36 and 4.37 give a set of images representative of the entire culture surface of each analyzed well. Figure 4.37 compares a series of images of wells, using the same application of filters, to determine the relative signatures of cells, QD constructs, and phospholipids specific to each filter: Alexa Fluor 488, PI, Qdot565, and TDI. Each image contains structures that fluoresced red. Only row B, wells exposed to QD constructs, contains a bright yellow structure in images B1 and B3, corresponding to the same position of the structure identified in $\mathrm{B} 2$ as being a QD.
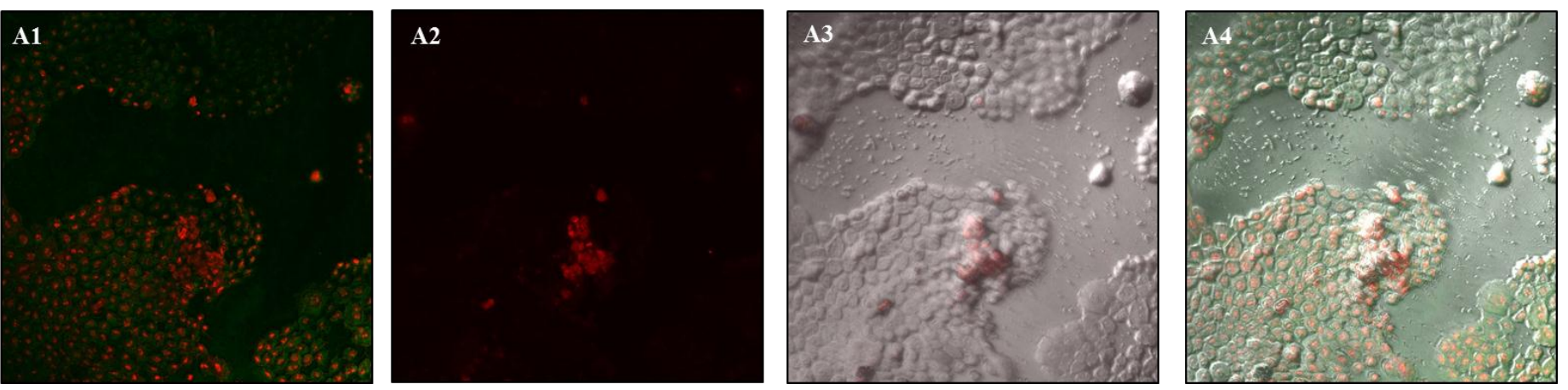

Figure 4.36 Series of confocal images taken of c2.5 (QD construct A 0.45 micron filter) Filters: A1 - Alexa Fluor 488 and PI; A2 - Qdot565; A3 - Qdot565 and TDI; A4 - Alexa Fluor 488, PI, and TDI 

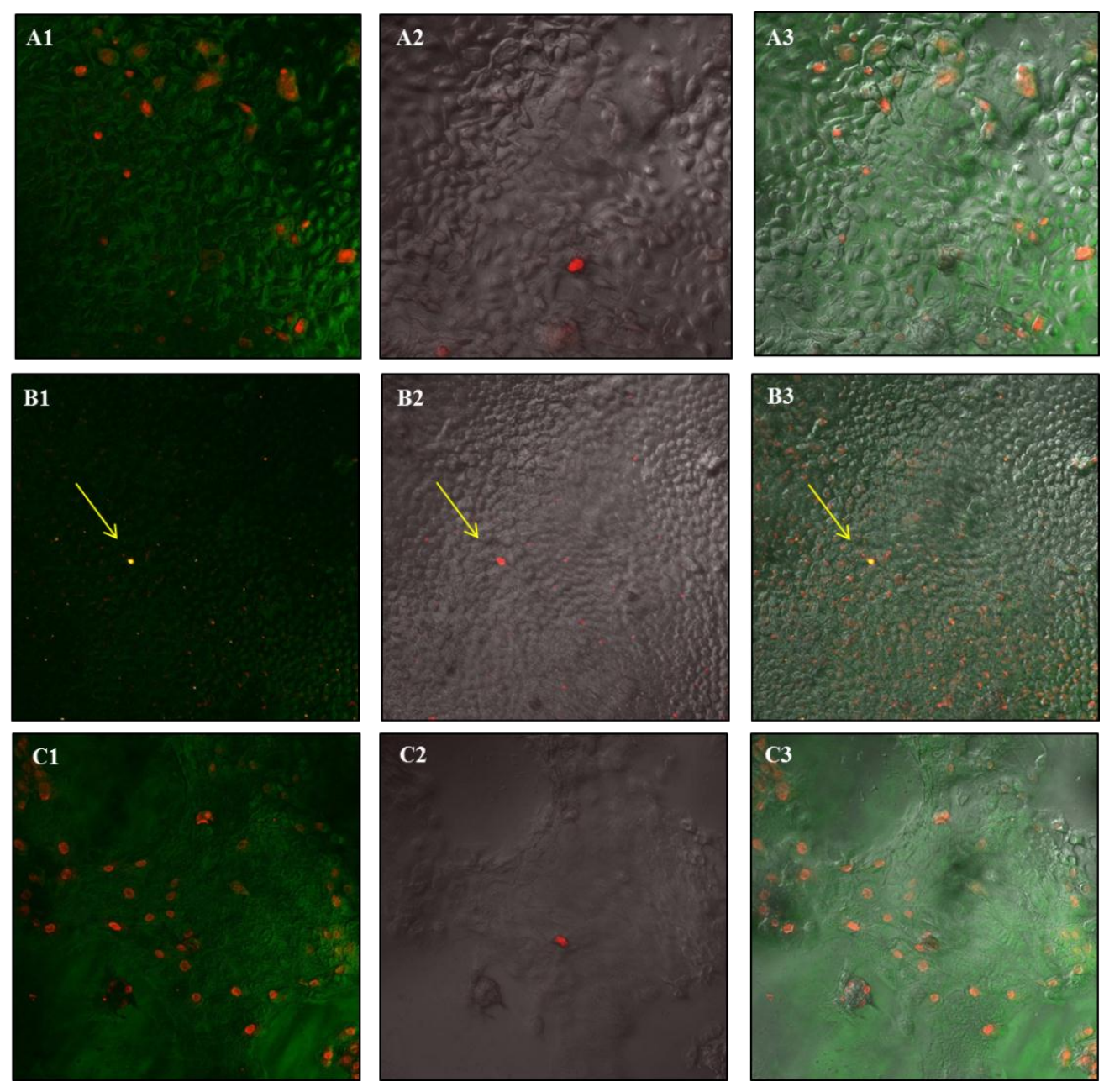

Figure 4.37 Comparison of confocal images: of c2.6, row A (keratinocyte media control); c2.7. row B (QD construct B 0.45 micron filter); c2.8, row C (phospholipid keratinocyte media 0.45 micron filter). Filters applied to each column: A1 - Alexa Fluor 488 and PI; A2 - Qdot565 and TDI; A3 - Alexa Fluor 488, PI, and TDI. The yellow arrow indicates a QD construct.

An additional series of $405 \mathrm{~nm}$ versus $488 \mathrm{~nm}$ images were conducted on the Hoechst stained cultures of well-set c. The subsequent images, given in figure 4.38 , return the same results as seen previously in the images taken for the Hoechst stained cultures of well-set $\mathbf{b}$. 

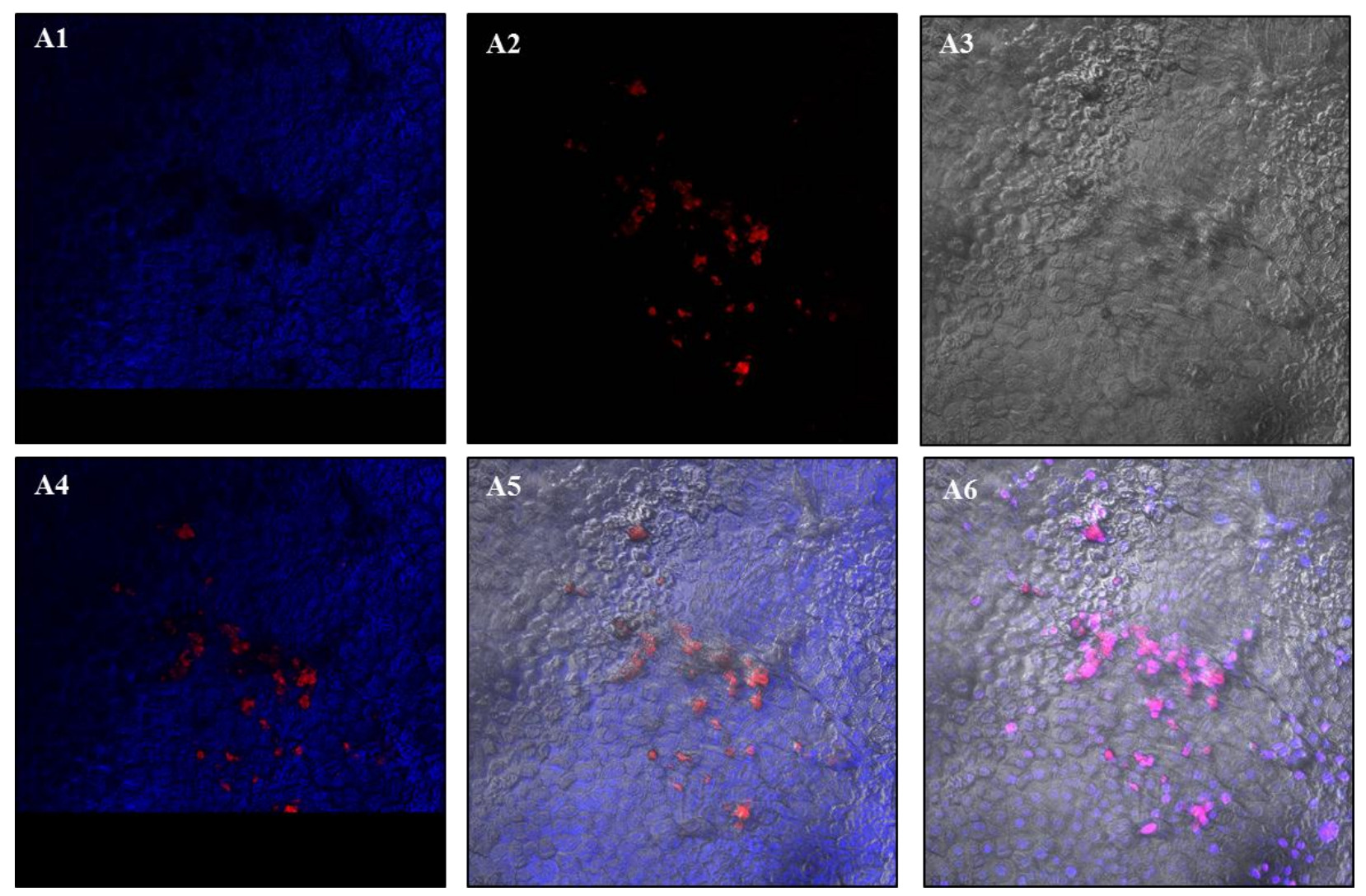

Figure 4.38 Series of confocal images taken of c1.1 (keratinocyte media control) comparing excitation wavelengths of 405 and 488nm; images A1-5 utilizing 488nm and image A5 utilizing 405nm. Filters applied to each column: 1- DAPI; 2 - Qdot565; 3 - TDI; 4 - DAPI and Qdot565; 5 - DAPI, Qdot565, and TDI; 6 - DAPI, Qdot565, and TDI.

\subsection{Discussion}

\subsubsection{QD Construct Introduction into Keratinocyte Cell Cultures}

\subsubsection{QD Construct Control and Test Solutions - RI}

Each step in the process for creating the QD constructs had an effect on the CMW of the solution. The encapsulation of the QDs in the phospholipid micelles and the filtering of the subsequent solution both resulted in a decrease CMW. There are two possible explanations for this effect: the QDs inside the micelles have a decreased diameter and/or the phospholipids absorb a portion of the energy from the excited electron in the form of phonons, thus reducing the energy of the emitted photon. As described in chapter one, a decrease in the size of QDs will result in a 
decrease in the emission wavelength. It could be possible that during the encapsulation process the QDs de-aggregate into smaller particles. However, knowing that exposure to heat, which the encapsulation process includes, typically promotes aggregation of QDs and thereby increases emission wavelengths; it seems unlikely that the decreased emission wavelength is the result of the size of the QD being diminished. The more likely reason is that the presence of the phospholipids surrounding the QDs removes energy. Filtering of the test solutions also resulted in a shift of noticeable shift in wavelength and intensity; again the result being a decrease. Filtering inevitably removes a significant proportion of the QD constructs present; the cause of the decrease in intensity. The QD constructs preferentially removed first would be the larger constructs. Removal of the larger constructs would mean removal of the larger QDs and therefore removal of the source of the larger emission wavelengths, all contributing to the decrease in CMW of the filtered solution.

\subsubsection{QD Construct Control and Test Solutions - R2}

The effect of a more coagulated encapsulation mixture, like the one produced during the encapsulations performed in R2, increased the difficulty for achieving an even distribution of QD constructs within the keratinocyte media; consequently, less of the actual QD constructs passed through the filter. The coagulation may also have led to less QDs actually being encapsulated, causing the majority of what was passed through the filter to be congealed phospholipids without any QDs. Comparison of the peaks for the filtered QD construct solutions, of R1 and R2, showed that the R2 0.45 micron solution to be five times less intense than the R1 0.45 micron QD construct solution. This difference would seem to support the conclusion that the encapsulation of the R2 solutions was not as effective as the R1 samples and that in future encapsulation proceedings, measures should be ensure a less viscous end product. The dilution of the QD construct test 
solutions, during the encapsulation process, using the native keratinocyte media would be an effective method for achieving this.

\subsubsection{Optical Imaging $-R 1$}

The identity of the orbs that appeared in the keratinocyte cell cultures 24 hours after the introduction of the test solutions remains unknown. An extensive literature and internet search of common keratinocyte cell culture contaminants, as well as typical cell anomalies, did not return any results that appeared similar in appearance or description. Based on the spherical and concentric appearance of the orbs it was proposed by Dr. Kristen O'Halloran Cardinal that they may in fact have been dead cells, but that without viewing them first hand it could be some contaminant. The confocal images of the same cultures did not reveal an obvious presence of the orbs, however, in the cultures that had been exposed to the QD construct test solutions, it was noted that some of the structures that the QD constructs had attached to, could in fact be the orbs. The image in Figure 4.39 is an example of the QD construct attachment to a structure similar to what was previously described.

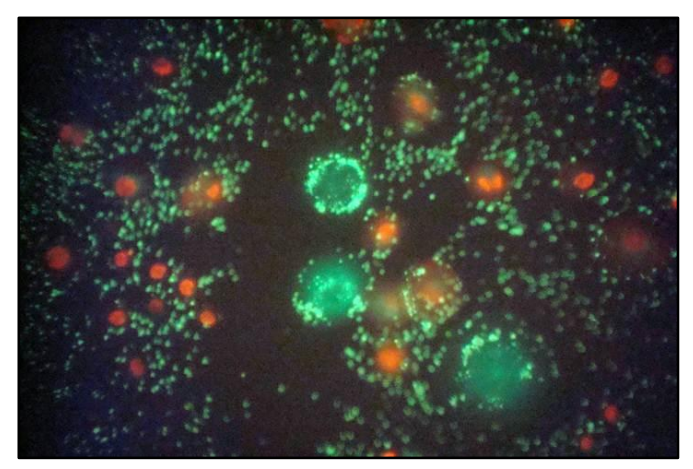

Figure 4.39 Taken with a digital camera through the eyepiece of the microscope with the wide field fluorescent laser applied to well b3.10 (QD PL krt filtered).

The images of the seemingly burst cell in Figure 4.22, whether or not the cell had actual swollen up to the point of bursting, can be positively identified as a cell. The interesting quality to notice in 
that image is that there is still a considerable amount of green visible around the cell from the presence of the Calcein stain. This indicates that the cell was alive at the time of exposure to the Calcein. The considerable amount of QD construct attachment around the periphery, and in the interior live portion of the cell, supports the hypothesis that the constructs were able to permeate the cell membrane. It does not however, provide substantial information from which to conclude when the constructs permeated the membrane. One explanation is that perhaps the constructs invade live cells causing cell death, and at the point of cell death, as enzymatic activity stops and the presence of enzymes declines, the constructs no longer have a point of attachment and therefore move out and away from the cells along with the cellular debris. This explanation could justify the lack of QD constructs within the completely red cells and the varying degree of their presence with respect to the amount of fluorescing Calcein.

The confocal images of the wells containing the unfiltered QD construct test solutions show minimal evidence of attachment of QD constructs to culture components. The difference between the images of the filtered and the non-filtered QD construct test solutions provide evidence for the benefits of filtering the solution as a method for increasing the interaction between the cells and the QD constructs. The nature of the phospholipids is such that when in large amounts they tend to aggregate forming particles too large to effectively interact with the cells. The series of images applying Qdot565 filter and the Alexa Fluor488 and PI filters for the different focal planes of the same field of view indicate that the larger aggregates of QD constructs, though not appearing to permeate any of the cells, appear to group around the cells; this is especially apparent in images A4 and B4 of Figure 4.27 .

\subsubsection{Optical Imaging $-R 2$}

The appearance of the cell cultures used in R2 were, on average, more confluent with larger numbers of cell colonies than the cultures in R1. The appearance of the cultures, with respect to 
contamination, was similar both before and after the administration of the test solutions. Taking an extended series of images of the cells as viewed through the microscope, made the process of comparing anomalies found in the confocal images with their possible source in the optical images.

\subsubsection{Keratinocyte Cell Viability and Cell-QD Construct Interaction - R1}

Confocal images with different focal depths illustrate the degree of and effect of the L/D stain, indicating that many of the cells were alive at the time of the L/D stain was introduced to the culture. Additionally different focal planes provide a method for uncovering the effects of the EthD-1 stain at the earliest stages by looking at components of the cell both proximal and distal to the nucleus. A factor worth noting is that although all of the wells in sub-set b1.1-4 were exposed to the L/D stain at the same time, b1.3 was the third well in the sub-set to be imaged and therefore had a longer exposure time than b1.1 would have. Averaging the time it took to acquire a series of images for each well, well b1.3 had a minimum exposure of 25 minutes.

Direct comparison of the confocal images acquired for b1.1 versus those of b1.3 would appear to support the hypothesis that the L/D stain may itself have a detrimental effect on the viability of the keratinocyte cells and as a result be a factor in the ratio of dead to live cells, however, the particular test solutions that each well was exposed to should also be taken into consideration. Well b1.1 contained a control solution of fresh keratinocyte media whereas well b1.3 contained keratinocyte media that had been mixed with $\mathrm{CHCl}_{3}$, heated at $55^{\circ} \mathrm{C}$, and then presumably had the $\mathrm{CHCl}_{3}$ evaporated off. It was noticed that the edges of the culture wells had a greater density of cell coverage, less cell death via EthD-1 stain, but also less cell-QD interaction.

\subsubsection{Keratinocyte Cell Viability and Cell-QD Construct Interaction - R2}

Two additional characterization techniques were employed in $\mathrm{R} 2$ that had not been used in R1: H/H staining and DIC imaging. Both of these proved to be helpful in distinguishing the presence of the cells and the interaction of the QD constructs with the cells. Unfortunately, the QD 
construct solutions of R2 were not to the standard of the solutions used in R1. Throughout all of the confocal imaging it was obvious that there was a decreased presence of QD constructs in the cell culture relative to the concentration of QD constructs in the solutions used in R1. Figure 4.31 of well a2.5 is especially effective at illustrating the difference between the two sets of QD-construct test solutions; only a single QD construct is distinguishable. Use of Hoechst stain was applied as a method for decreasing possibility for the misidentification of QD construct attachment to cells in the likelihood of a reoccurrence of the QD interaction with possible non-cell entities as seen in the R1 trials.

A difficulty encountered during the confocal imaging of cells in R2 that was not present in R1 was a result of the four-well slides the cells had been cultured in. To acquire clear images of the cells using the confocal microscope it was necessary that there only be a very thin barrier between the cells and the objective lens. The thicker specimen slide base that the four-well in R2 were attached to prevented this. Among the alterations that had to be made to image the cells was aspiration of the solution in each well. Aspiration of the solutions could have very likely compromised the environment of the cultures, with respect to imaging, relative to what was seen in $\mathrm{R} 1$. The preparation of the slide also introduced extra variables such as dehydration of the samples, removal of QD constructs that were previously interacting with the cells, and shock to the cells as a consequence of the removal process of the rubber septum. The application of the razor blade for well-sets $\mathbf{b}$ and $\mathbf{c}$, over the metal spatula used for well-set $\mathbf{a}$, greatly decreased the time and damaged, and increased the degree of complete removal of the rubber septum. This more complete removal provided a better surface for the cover slip to attach to, thereby yielding better images.

Application of the Qdot565 filter, for imaging the non-QD construct wells, aided in assessing unknown factors which might be presumed to be QD constructs in the associated wells, 
but were actually artifacts related to some other presence within the culture. A subsequent effect of using the Hoechst stain was the false registration of QD constructs. The excitation/emission wavelength ranges of the Hoechst stain and the QD constructs were very similar. As a consequence of this similarity the QD565 filter caught a portion of the emission form the Hoechst stain. This emission was then falsely registered as a QD construct signature when in fact no QD constructs were present; a circumstance that was supported by the application of the Hoechst and Qdot565 filters on wells that had not been exposed to any QD constructs. According to the image results using the DAPI and Qdot565 filters on wells that were sans QDs, QD constructs were present. Fortunately, the presence of the fluorescence in the image was not the sole factor for determining the presence of the QD-constructs; the shape and structure of the image was also a method for identification. The constructs, throughout all of the images in both sets of trials, consistently displayed a distinct structure. This structure varied depending on the degree of filtration of the QD construct solutions, but was none the less distinguishable relative to the keratinocyte cells. A Hoechst stain affect was to use a higher excitation wavelength. Using an excitation wavelength of $488 \mathrm{~nm}$, rather than $405 \mathrm{~nm}$, provided a method for exciting any QD constructs that may have been present that was outside the optimal range of excitation wavelengths needed to excite the Hoechst stain. 


\section{Chapter 5 - Discussion and Conclusions}

\subsection{Overview and Summary}

Cancer is a prevalent and devastating health issue and the second leading cause of death in the United States (2). The most common type of cancer found in the United States is skin cancer (4). Early and accurate detection is the most reliable way to ensure a positive outcome and the ultimate survival of the patient (5). Accurate determination of the presence of cancerous cells in a biopsy is especially difficult at the early stages when only a small percentage of cells in the biopsied mass show the morphological traits associated with being cancerous. This circumstance often results in a false negative (FN), delaying the necessary treatment until the cancer has reached a more developed stage (7). For this reason alternative methods for the identification of individual cancer cells within a larger population of healthy cells is required; functionalized QD constructs can be this alternative method. Already QDs have been investigated in in vitro and in vivo studies as a viable method for the detection of cancerous cells within prostate, colon, and breast tissue cultures $(9,10)$. The extensive investigations that are being conducted into the applications of QDs for use as biologic tags and vehicles for targeting cancer, demonstrate the value that they offer.

Currently the CdSe/Zn QDs being synthesized at Cal Poly generate QDs with a wide range of CMWs, intensities, and FWHMs. This ability to synthesize such a range of products demonstrates the potential for developing a procedure for the consistent optimization of multiple QDs that can then be used in specific biologic applications. By addressing some of the QD synthesis challenges encountered and described in chapter 2 , such as temporal wavelength shifts and lowered intensities post-ZnS shelling, great strides will be made toward achieving a reliable method for synthesis of QDs of consistent CMW and intensity. 


\subsection{Challenges, Limitations, and Future Work}

Of the three different sets of QD construct test solutions created, two of which are detailed in this thesis, the third solution presented challenges during the filtration process. The initial encapsulation mixture had an increased viscosity and decreases diffusion within the keratinocyte media into which it had been suspended. This along with the introduction of the 0.2 micron filter in the third set of trials resulted in QD-construct test solutions of decreased concentration. The confocal images acquired of the keratinocyte cultures associated with this set of QD construct solutions showed decrease in the presence of QDs and there interaction with the keratinocyte cells. In future experiments every trial should contain test solutions that have been filtered with $0.8,0.45$, and 0.2 micron filters to determine the degree to which each filter causes an effect on the QD construct concentration and subsequent cell-QD construct interactions.

Working with fluorescent stains such as Calcein, EthD-1, and Hoechst presented challenges for imaging of the culture trials. As a result of overlapping emission wavelengths the filters applied to acquire the confocal images could not be used in concert with one another. the QD filter had to be used separately form the Alexa Fluor488 and PI filters for detecting the L/D stain and the excitation wavelength corresponding to the Qdot565 filter had to be exchanged for a higher wavelength so as to not inadvertently excite the Hoechst stain and misidentify it as a QD. Although all of these challenges were eventually overcome, it was by trial and error. In each of the trials a new and useful characterization mechanism was added providing a more complete picture of the effects of the QD construct test solutions on the keratinocyte cells. In future trials it would be beneficial to add additional staining techniques for the determination of cell proliferation as well as cell viability.

A long term study of the keratinocyte cell-QD construct interaction was not possible as a result of the constraints related to maintaining an aseptic environment. Most of the cell cultures 
showed an initial amount of contamination pre-introduction of the test solutions and in some instances this small amount of initial contamination became a prominent presence in the cultures by the end of the 24 hour test period. Not only did the presence of contamination often cloud the view, it also clouded the conclusions as to what was the primary factor affecting cell viability; the QD test solutions or the contamination. Additionally some of the confocal images acquired of the resulting keratinocyte cell-QD construct interactions presented questions as to what the QDs were attaching to. Many of the structures that were initially assumed to be keratinocyte cells were, after further trials had been conducted, later called into question. It was concluded that there was evidence of QD construct attachment to both keratinocyte cells and the unidentified structures (USs); even more so to the unidentified structures. This called into question why the QD constructs seemed to have a greater affinity for the USs; a question that has remained unanswered. In future, if a QD construct solution can be synthesized that is capable of being filtered through the 0.2 micron filter, which is necessary to prevent contamination, without decreasing the concentration of QD constructs within the solution, it may be possible to conduct trials with longer time points and less contamination. Ultimately, once the procedure for encapsulating and filtering the QD construct test solutions has been optimized, transition in to more complex biologics, such as EpiDerm skin substitutes, will be the next adventure.

\subsection{Conclusion}

Although there are many journal articles describing and reviewing a variety of procedures for the solubilization and phospholipid functionalization of CdSe/ZnS QDs for use in a biologic, this thesis describes the first attempt of such an endeavor at Cal Poly. This method for synthesizing QD constructs has great potential for applications where cellular tagging is desired or where monitoring the migration of cellular components in real time is of interest. The current protocol 
being used at Cal Poly for the synthesis CdSe/ZnS QDs has proved to be a sufficient method for supplying QDs that are compatible with phospholipid encapsulation. Further modifications of both the $\mathrm{CdSe} / \mathrm{ZnS}$ QD synthesis and optimization of the encapsulation protocol developed here, will provide a multitude of future applications only limited by imagination. 


\section{LIST OF REFERENCES:}

1. Siegel, R., Naishadham, D. and Jemal, A. (2012), Cancer statistics, 2012. CA: A Cancer Journal for Clinicians, 62: 10-29. doi: 10.3322/caac.20138

2. Mayo Clinic. Cancer(2012). Retrieved form http://www.mayoclinic.com/health/cancer/DS01076

3. Jemal, A., Bray, F., Center, M. M., Ferlay, J., Ward, E. and Forman, D. (2011), Global cancer statistics. CA: A Cancer Journal for Clinicians, 61: 69-90. doi: 10.3322/caac.20107

4. National Cancer Institute at the National Institutes of Health. National Cancer Institute (NCI) What You Need to Know About Melanoma and Other Skin Cancers. (NIH Publication No. 10-7625) Retrieved from http://www.cancer.gov/cancertopics/wyntk/skin.pdf

5. National Cancer Institute. National Institutes of Health. (Jan. 2012). Cancer Screening Overview . Retrieved from http://www.cancer.gov/cancertopics/pdq/screening/overview/HealthProfession al/page1\#Section_15

6. Bartolome et al. The Chemokine Receptor CXCR4 and the Metalloproteinase MT1-MMP Are Mutually Required during Melanoma Metastasis to Lungs. AJP February 2009, Vol. 174, No. 2

7. Veenstra, H. J., M. J. W. M. Wouters, et al. "Less false-negative sentinel node procedures in melanoma patients with experience and proper collaboration." Journal of Surgical Oncology 104(5): 454-457.

8. Rathore, Saif S. et al. Mandated Coverage for Cancer-Screening Services: Whose Guidelines Do States Follow? American journal of preventive medicine [0749-3797] (2000), 19:2 
9. Gao, X., Y. Cui, et al. (2004). "In vivo cancer targeting and imaging with semiconductor quantum dots." Nat Biotech 22(8): 969-976.

10. Smith, R., S. L. Sewell, et al. (2008). "Proximity-activated nanoparticles: in vitro performance of specific structural modification by enzymatic cleavage." Int $\mathbf{J}$ Nanomedicine 3(1): 95-103.

11. Fan, H., E. W. Leve, et al. (2005). "Surfactant-Assisted Synthesis of Water-Soluble and Biocompatible Semiconductor Quantum Dot Micelles." Nano Letters 5(4): 645-648.

12. Carion, O., B. Mahler, et al. (2007). "Synthesis, encapsulation, purification and coupling of single quantum dots in phospholipid micelles for their use in cellular and in vivo imaging." Nat. Protocols 2(10): 2383-2390.

13. Dubertret, B., P. Skourides, et al. (2002). "In vivo Imaging of Quantum Dots Encapsulated in Phospholipid Micelles." Science 298(5599): 1759-1762.

14. A.D.A.M. Medical Encyclopedia. (2010) Tumor Mass; Neoplasm. Retrieved from http://www.ncbi.nlm.nih.gov/pubmedhealth/PMH0002287/

15. Stanford Medicine. (2012). Genetics and Cancer: How Genes Cause Cancer. Retrieved from http://cancer.stanford.edu/information/geneticsAndCancer/genesCause.html

16. Kurschat, P. et al. Identification of activated matrix metalloproteinase-2 (MMP-2) as the main gelatinolytic enzyme in malignant melanoma by in situ zymography. $\mathrm{J}$ Pathol 2002; 197: 179-187.

17. Fidler, Isaiah. The pathogenesis of cancer metastasis: the 'seed and soil' hypothesis revisited. Nature Reviews: Cancer. 2002; Vol.3

18. Folkman, Judah. How Is Blood Vessel Growth Regulated in Normal and Neoplastia Tissue? Cancer Res 1986;46:467-473. 
19. Weis et al. Endothelial barrier disruption by VEGF-mediated Src activity potentiates tumor cell extravasation and metastasis. The Journal of Cell Biology, Vol. 167, No. 2, October 25, $2004223-229$

20. Liotta, Lance. Tumor Invasion and Metastasis_Role of the Extracellular Matrix. Cancer Res 1986;46:1-7.

21. National Cancer Institute at the National Institutes of Health. (2012). Cancer Causes and Risk Factors. Retrieved from http://www.cancer.gov/cancertopics/causes

22. National Cancer Institute at the National Institutes of Health. (2012). BRCA1 and BRCA2: Cancer Risk and Genetic Testing. Retrieved from http://www.cancer.gov/cancertopics/factsheet/Risk/BRCA

23. Basal cell carcinoma. (Feb. 2008). A.D.A.M. Medical Encyclopedia online. Retrieved from http://www.ncbi.nlm.nih.gov/pubmedhealth/PMH0001827/

24. Saladin, Kenneth S. (2007) Anatomy and Physiology: the Unity of Form and Function $4^{\text {th }}$ Ed. McGraw-Hill. New York, New York

25. Squamous cell carcinoma. (July 2011). A.D.A.M. Medical Encyclopedia online. Retrieved from http://www.ncbi.nlm.nih.gov/pubmedhealth/PMH0001832/

26. Melanoma. (July 2011). A.D.A.M. Medical Encyclopedia online. Retrieved form http://www.ncbi.nlm.nih.gov/pubmedhealth/PMH0001853/

27. NASA. (2012).Measuring the Electromagnetic Spectrum. Retrieved from http://imagine.gsfc.nasa.gov/docs/science/know_11/emspectrum.html

28. Public Health. (2012). Europa. [Illustration of Electromagnetic Spectrum]. Retrieved from http://ec.europa.eu/health/opinions/en/energy-saving-lamps/figtableboxes/lightspectrum.htm 
36. NASA. (2012). The Electromagnetic Spectrum- Ultraviolet Waves. Retrieved from http://science.hq.nasa.gov/kids/imagers/ems/uv.html

37. US Environmental Protection Agency. (2012). UV Radiation. Retrieved from http://www.epa.gov/sunwise/doc/uvradiation.html

38. Gruijl, Frank R. (2002) Photocarcinogenesis: UVA vs. UVB Radiation. Skin pharmacology and physiology (15:5).[1660-5527]

39. Tortora, Gerard J., Derrickson, Bryan H. Principles of Anatomy and Physiology $13^{\text {th }}$ Ed. (n.c.) Wiley, Hohn and Sons, Inc. Retrieved from http://www.imperial.edu/ thomas.morrell/cha_5_tortora_integument.htm

40. Koeppen, Bruce M. and Bruce A. Stanton. (Eds.). (2010). Berne and Levy: Physiology (6 $6^{\text {th }}$ Ed.) Philadelphia, PA: Mosby Elsevier Inc.

41. Phospholipids. (2012). Natural Products: Lipids. Retrieved from http://www2.chemistry.msu.edu/faculty/reusch/VirtTxtJml/lipids.htm

42. Brown, Bernard S. (1996) "The Biochemistry Across the School Curriculum Group (BASC): Biological Membranes” London, The Biochemical Society.

43. Taylor, John R., Zafiratos, Chris D., Dubson, Michael A. (2004). Modern Physics for Scientists and Engineers $2^{\text {nd }}$ Ed. New Jersey, Pearson Prentice Hall.

44. Nave, Rod. (n.d.). Band Theory of Solids. Hyper Physics. Retrieved from http://hyperphysics.phy-astr.gsu.edu/hbase/solids/band.html

45. Smith, William F., Hashemi, Javad. (2006). Foundations of Materials Science and Engineering $5^{\text {th }}$ Ed. New York, McGraw Hill.

46. Bruice, Paula Y. (2007). Organic Chemistry $5^{\text {th }}$ Ed. New Jersey: Pearson Prentice Hall.

47. Taylor, John R., Zafiratos, Chris D., Dubson, Michael A. (2004). Modern Physics for Scientists and Engineers $2^{\text {nd }}$ Ed. New Jersey, Pearson Prentice Hall. 
48. Semiconductors. (Sept. 2002). Nobelprize.org [Atomic orbitals image adapted from Sally Con cartoon] Retrieved from http://www.nobelprize.org/educational/physics/semiconductors/index.html

49. Freedman, Roger A., Young, Hugh D. (2004). University Physics with Modern Physics $11^{\text {th }}$ Ed. New York, Pearson Addison Weasley.

50. Chenming-Hu. (Feb. 2009) Electrons and Holes in Semiconductors. Retrieved from http://www.eecs.berkeley.edu/ hu/Chenming-Hu_ch1.pdf

51. Rosenthal, S. J., J. C. Chang, et al. "Biocompatible Quantum Dots for Biological Applications". Chemistry \& biology 18(1): 10-24.

52. Liboff, Richard L. (2003). Introductory Quantum Mechanics $4^{\text {th }}$ Ed. New York: Addison Wesley.

53. L.O. Cinteza,. (2011). "Multifunctional nanosystems for cancer theragnostics". Biomedical Optics and Medical Imaging. SPIE Newsroom. 10.1117/2.1201103.003432.

54. Rivera, Erin M., et al., Imaging heterostructured quantum dots in cultured cells with epifluorescence and transmission electron microscopy. Journal of Colloidal Quantum Dots/Nanocrystals for Biomedical Applications. Vol. 7909, 79090N1-12. 2011.

55. Chan, W. C. W., D. J. Maxwell, et al. (2002). "Luminescent quantum dots for multiplexed biological detection and imaging." Current Opinion in Biotechnology 13(1): 40-46.

56. Cinteza, Ludmila Otilia. Quantum dots in biomedical applications: advances and challenges. Journal of Nanophotonics. Vol. 4 (042503), 2010.

57. Klostranec, J. M., Q. Xiang, et al. (2007). "Convergence of Quantum Dot Barcodes with Microfluidics and Signal Processing for Multiplexed High-Throughput Infectious Disease Diagnostics." Nano Letters 7(9): 2812-2818. 
58. Sewell, Sarah L., et al., Synthesis and enzymatic cleavage of dual-ligand quantum dots. Materials Science and Engineering C. Vol. 29, 1428-1432. 2009.

59. Smith, Adam R., et al., Proximity-activated nanoparticles: in vitro performance of specific structural modification by enzymatic cleavage. International Journal of Nanomedicine. Vol. 3, No. 1, 95-103. 2008.

60. Myles, Charles W. (Dec. 2012). Overview of Quantum Confinement. [Power Point Slides] Retrieved from www.phys.ttu.edu/ cmyles/Phys5335/Lectures/12-2-10.ppt

61. R. Mazurczyk. (n.d.).Semiconductor Superlattices. Centre for Molecular and Macromolecular Studies, Polish Academy of Sciences, Sienkiewicza 112, 90-363Lodz, Poland

62. Lee, Corissa. (April 2010). Quantum Dots. [Power Point Slides] Retrieved from http://mechse.illinois.edu/media/uploads/course_websites/lee_quantumdots.20100425.4b d4c1c5be8fe7.73485281.pdf

63. Miller, D. A. B., D. S. Chemla, et al. (1984). "Band-Edge Electroabsorption in Quantum Well Structures: The Quantum-Confined Stark Effect." Physical Review Letters 53(22): 2173-2176.

64. Drummen, G. "Quantum Dots - From Synthesis to Applications in Biomedicine and Life Sciences." International Journal of Molecular Sciences 11(1): 154-163.

65. Vuckovic, Jelena. (May 2011) Quantum Nanophotonics: Quantum Science Thought Leaders Series. Retrieved from http://www.stanford.edu/ arkam/QuantumNanophotonics.pdf

66. Physics and Chemistry Nanostructures Group. (2012). Top-down Versus Bottom-up. Retrieved form 
http://www.nano.ugent.be/index.php?option=com_content\&task=view\&id=100\&Itemid= 88

67. Junkin, M., J. Watson, et al. (2009). "Template-Guided Self-Assembly of Colloidal Quantum Dots Using Plasma Lithography." Advanced Materials 21(12): 1247-1251.

68. Malik, S., C. Roberts, et al. (1997). "Tuning self-assembled InAs quantum dots by rapid thermal annealing." Applied Physics Letters 71(14): 1987-1989.

69. Petroff, P. M., A. Lorke, et al. (2001). "Epitaxially Self-Assembled Quantum Dots." Physics Today 54(5): 46.

70. Chan, W. C. W., D. J. Maxwell, et al. (2002). "Luminescent quantum dots for multiplexed biological detection and imaging." Current Opinion in Biotechnology 13(1): 40-46.

71. Sigma Aldrich. (2007). Advanced Applications of Engineered Nano Particles. Material Matters. 2(1): 10-11. Retrieved from http://www.sigmaaldrich.com/etc/medialib/docs/Aldrich/Brochure/al_material_matters_v 2n1.Par.0001.File.tmp/al_material_matters_v2n1.pdf

72. Sengül, Hatice, and Theis, Thomas L.(2009). Life Cycle Inventory of Semiconductor Cadmium Selenide Quantum Dots for Environmental Applications. Chicago: William Andrew Inc.

73. Murray, C. B., D. J. Norris, et al. (1993). "Synthesis and characterization of nearly monodisperse $\mathrm{CdE}(\mathrm{E}=$ sulfur, selenium, tellurium) semiconductor nanocrystallites." Journal of the American Chemical Society 115(19): 8706-8715.

74. Nordell, K. J., E. M. Boatman, et al. (2005). "A Safer, Easier, Faster Synthesis for CdSe Quantum Dot Nanocrystals." Journal of Chemical Education 82(11): 1697.

75. Octadecane. (n.d.). G Love: Materials Selection. Retrieved from 
http://web.mit.edu/course/3/3.082/www/team2_s02/materials.html

76. Lipids. (n.d.). Florida International University Biochem Lab. Retrieved from

http://www2.fiu.edu/ bch3033/bch30331/pdf/LipidLab.pdf

77. Garting, Tommy. (April 2012). Isolation of CdSe Quantum Dot Nanoparticles. Retrieved from http://mrsec.wisc.edu/Edetc/nanolab/CdSe/index2.html

78. Fisher Scientific. (n.d.). Material Safety Data Sheet: Ethanol, Absolute.

79. Choloroform. (April 2000). Inchem. Retrieved from http://www.inchem.org/documents/icsc/icsc/eics0027.htm

80. Carl T. Dargitz, personal communication, April 11, 2012

81. ATCC. (2009).Primary Epidermal Keratinocytes; Normal, Human, Neonatal Foreskin Product Information Sheet for ATCC® PCS-200-010. Retrieved from www.atcc.org/attachments/7243.pdf

82. Mitotic Index. (n.d.). National Cancer Institue at the National Institutes of Health Retrieved from http://www.cancer.gov/dictionary?cdrid=386225

83. De Corte, P., G. Verween et al. (2012). "Feeder layer- and animal product-free culture of neonatal foreskin keratinocytes: improved performance, usability, quality and safety." Cell and Tissue Banking 13(1): 175-189, DOI: 10.1007/s10561-011-9247-3

84. Invitrogen. (2005). Live/Dead Viability/Cytotoxicity Kit for mammalian cells: Product Information. Retrieved from http://probes.invitrogen.com/media/pis/mp03224.pdf

85. Dr. Lily Laiho, personal communication,

86. Invitrogen. (n.d.). Calcein - High Purity Molecular Probe. Retrieved from https://products.invitrogen.com/ivgn/product/C481?ICID=search-product

87. Invitrogen. (n.d.).Ethidium homodimer-1Molecular Probe. Retrieved form https://products.invitrogen.com/ivgn/product/E1169?ICID=search-product 
88. Invitrogen. (n.d.). Hoechst 34580. Retrieved form

http://tools.invitrogen.com/content/sfs/manuals/mp21486.pdf

89. Dr. Kristin O’Halloran Cardinal, personal communication. May 9. 2012

90. Angell, Josh. (2011). Synthesis and Characterization of CdSe-Zn Quantum Dots for Increased Quantum Yield. Retrieved from Digital Commons at Cal Poly. 


\begin{tabular}{|c|c|}
\hline \multicolumn{2}{|c|}{ Appendix A - Abbreviations } \\
\hline BOs & black orbs \\
\hline $\mathrm{Cd}$ & cadmium \\
\hline $\mathrm{CdSe}$ & cadmium selenium cores \\
\hline $\mathrm{CHCl}_{3}$ & chloroform \\
\hline $\mathrm{H} / \mathrm{H}$ & Histochoice fixative and Hoechst stain \\
\hline krt & keratinocyte \\
\hline $\mathrm{L} / \mathrm{D}$ & live/dead cytotoxicity/viability Calcein/Ethidium homodimer-1 \\
\hline $\mathrm{MeOH}$ & methanol \\
\hline ODE & octadecene \\
\hline PL & phospholipids \\
\hline QD & quantum dots \\
\hline $\mathrm{R} 1$ & round one \\
\hline $\mathrm{R} 2$ & round two \\
\hline Se-TOP & seleniuom trioctylphosphine \\
\hline WFCs & white fluorescing CdSe cores \\
\hline $\mathrm{ZnS}$ & zinc sulfide quantum dots \\
\hline
\end{tabular}




\section{Appendix B - Protocols and Experimental Details}

\section{B.1 - Cal Poly Standard CdSe/ZnS Synthesis Protocol and Analysis Procedure}

The following CdSe/ZnS synthesis protocol and analysis procedure are the standard protocol and procedure documented by Josh Angell and used by Cal Poly's Biomedical Department.

\section{Appendix A: CdSe Quantum Dot Synthesis Reaction and Characterization Procedures}

The first part of this procedure outlines 3 different techniques (Purging, Syringing, \& Oil Bath Operation) which are required for quantum dot synthesis.

The second part contains instructions to create the selenium/cadmium precursors and then react them to synthesize CdSe quantum dots.

\section{Chemicals}

13 mg Cadmium Oxide Powder

$33 \mathrm{mg}$ Selenium Powder

$0.6 \mathrm{~mL}$ Oleic Acid (tech grade 90\%)

$15 \mathrm{~mL}$ Octadecene (tech grade 90\%)

$0.4 \mathrm{~mL}$ Trioctylphosphine (tech grade $90 \%$ )

Toluene and Acetone for cleanup

\section{Equipment}

2 - 50mL 14/20 1-neck or 3-neck Round Bottom Flasks

$2-1 \mathrm{~mL}$ Disposable Plastic Syringes

$2-3 \mathrm{~mL}$ Disposable Plastic Syringes

$1-5$ cc Glass Syringe, Luer Lock Tip

1 - Veterinary Tip, 18 gauge, 3" SS Needle

2 - Small Stir Bars

3 to $5-7.5 \mathrm{~mL}$ Borosilicate Vials

1 - Medium-Sized Beaker $(150 \mathrm{~mL})$

2 to 4 - Rubber Septa

Hot/Stir Plate with RTD Probe

Crystallization Dish

$\sim 200 \mathrm{~mL}$ - High Temperature Silicone Oil

Chemical Spatula

Kimwipes

Thermocouple

Analytical Balance

Stopwatch

Latex or Nitrile Gloves 


\section{Chemical Disposal}

All chemicals and equipment used during the quantum dot synthesis must be properly disposed of. Currently there is a vessel for liquid waste and a container for solid wastes such as needles, syringes, kimwipes, etc. which are stored in the yellow, hazardous chemical cabinet in the nanotech lab (Bldg. 41-205).

\section{Preparing Chemical Bottles}

1. Fill a clean dry bottle with the desired chemical.

2. Fit a rubber septum into the top of the bottle.

3. Either purge the solution if it is to be used immediately or store it for later use.

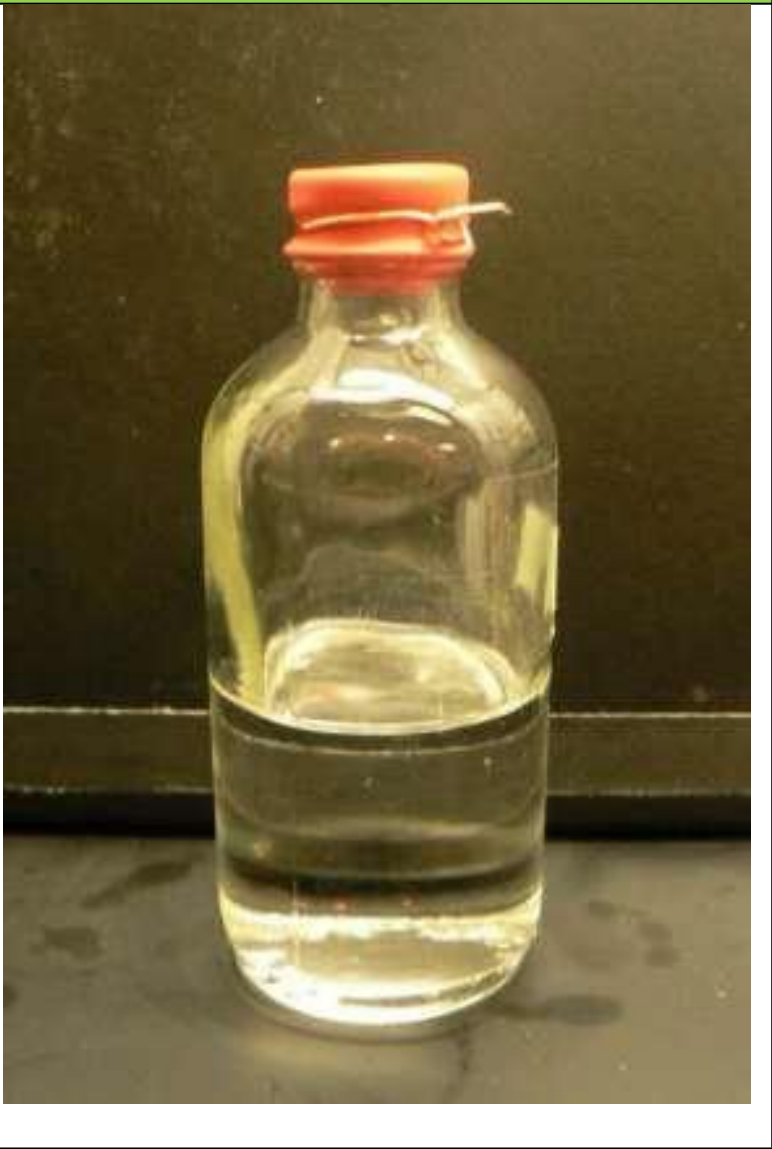




\section{Purging Procedure}

This procedure is to be used for any chemical and/or reaction vessel needing purging.

1. Fill a large beaker with water until it is $3 / 4$ full.

2. Lower $\mathrm{N}_{2}$ tank gas escape hose into beaker.

3. Fully open valve on top of tank.

4. Fully open left knob on valve.

5. Open middle knob on valve ("increase") until bubbles start to appear in the beaker. Maintain this gas flow throughout the procedure.

6. Insert $\mathrm{N}_{2}$ purge needle into center of septum. Make sure the needle is above the level of the liquid.
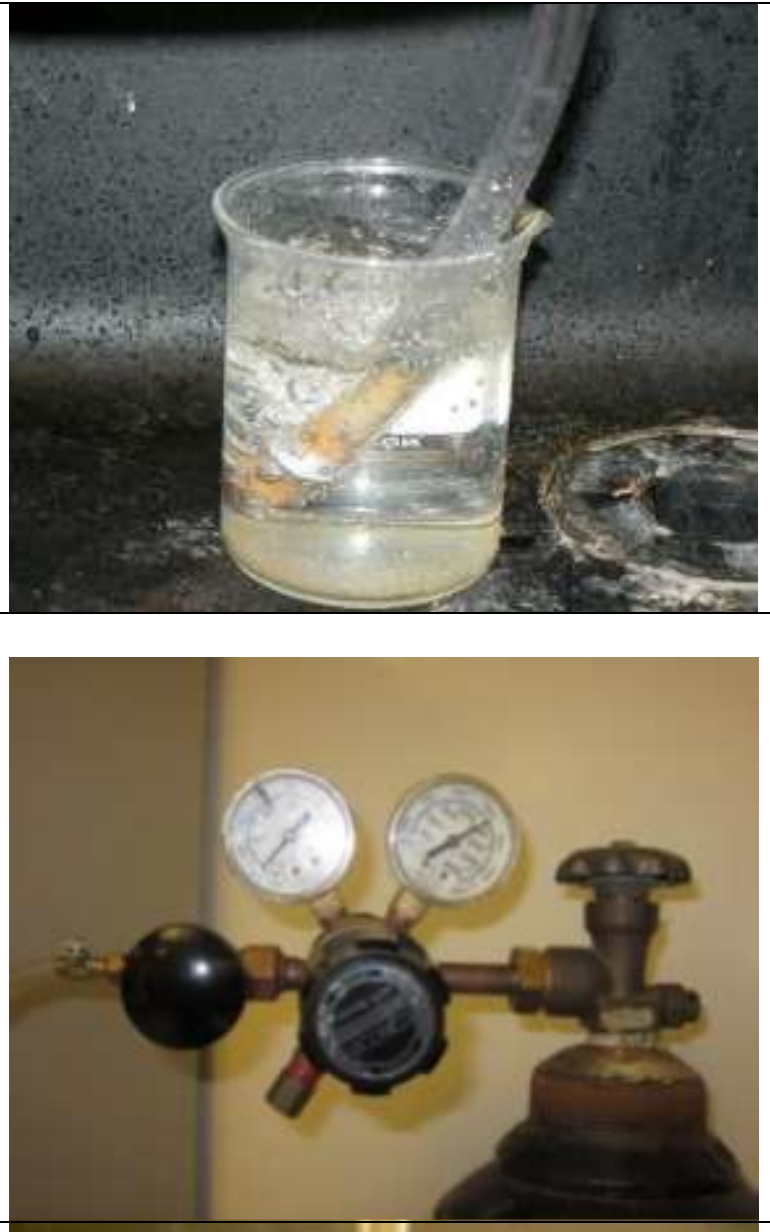


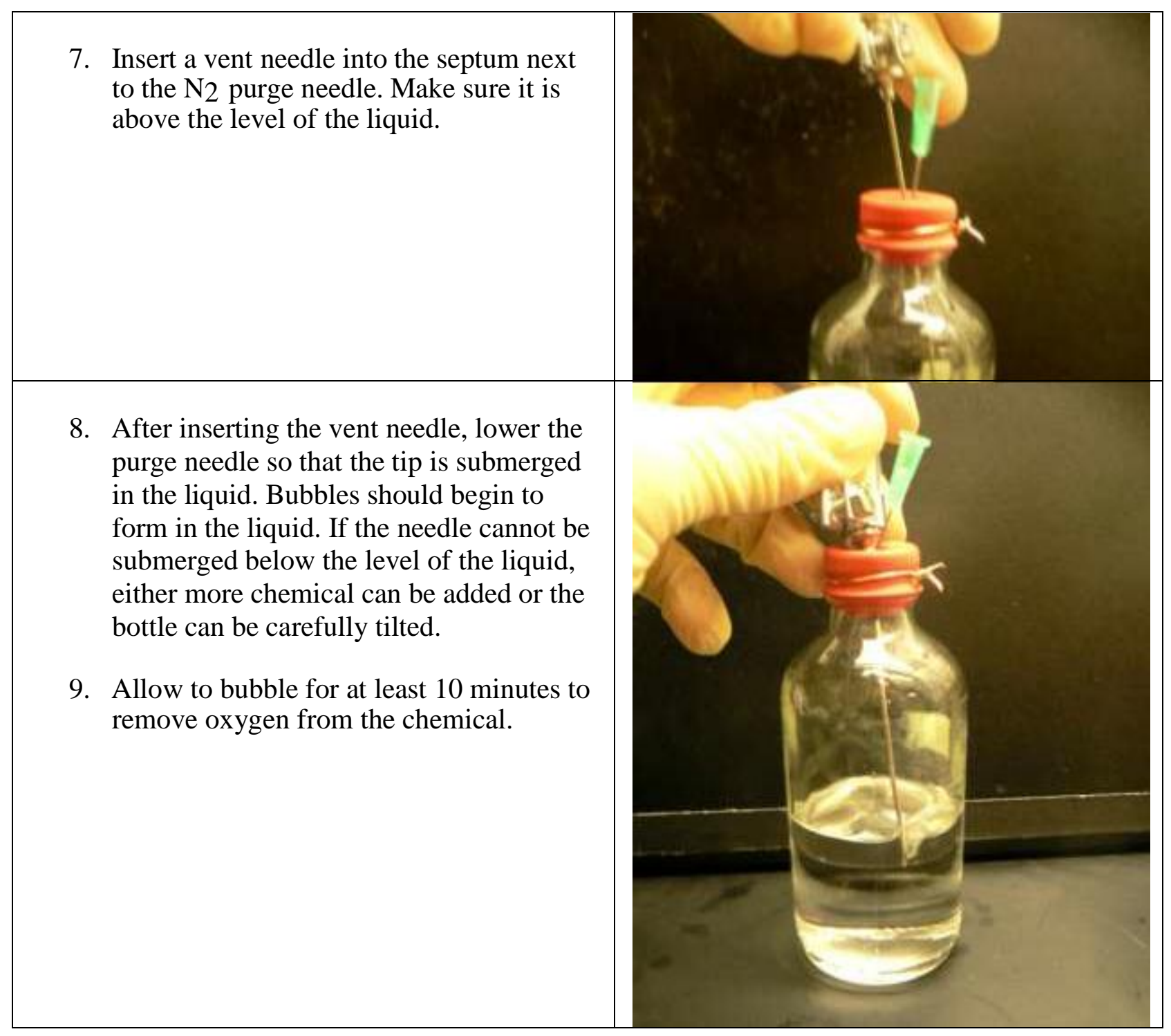




\section{Syringing Procedure}

This procedure is a method of transferring a chemical from one purged vessel into another without the introduction of oxygen. All solutions must be properly purged with $\mathrm{N}_{2}$ before using this procedure.

1. Remove a disposable plastic syringe from its packaging.

2. Pull the $\mathrm{N}_{2}$ purge needle up so that it is above the level of the liquid. No bubbles should be forming at this point.

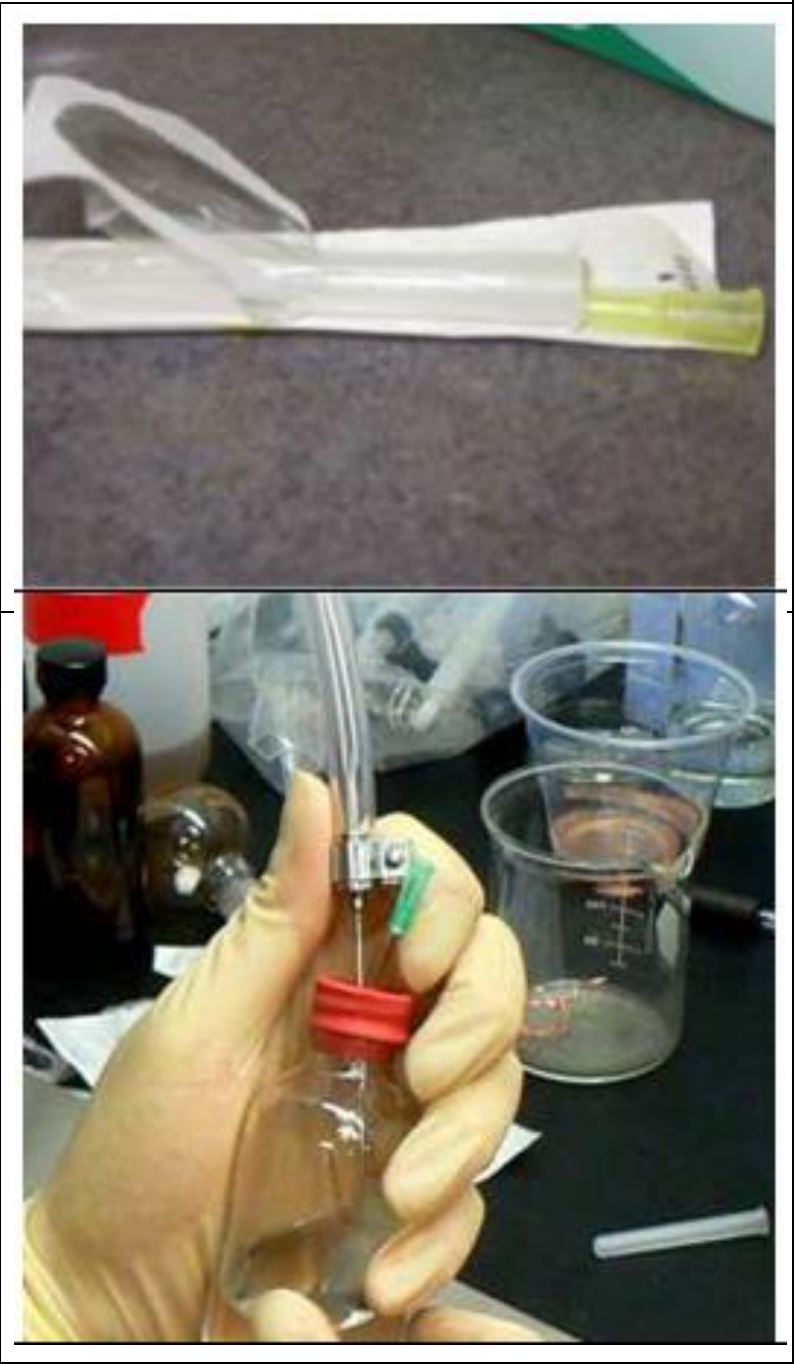




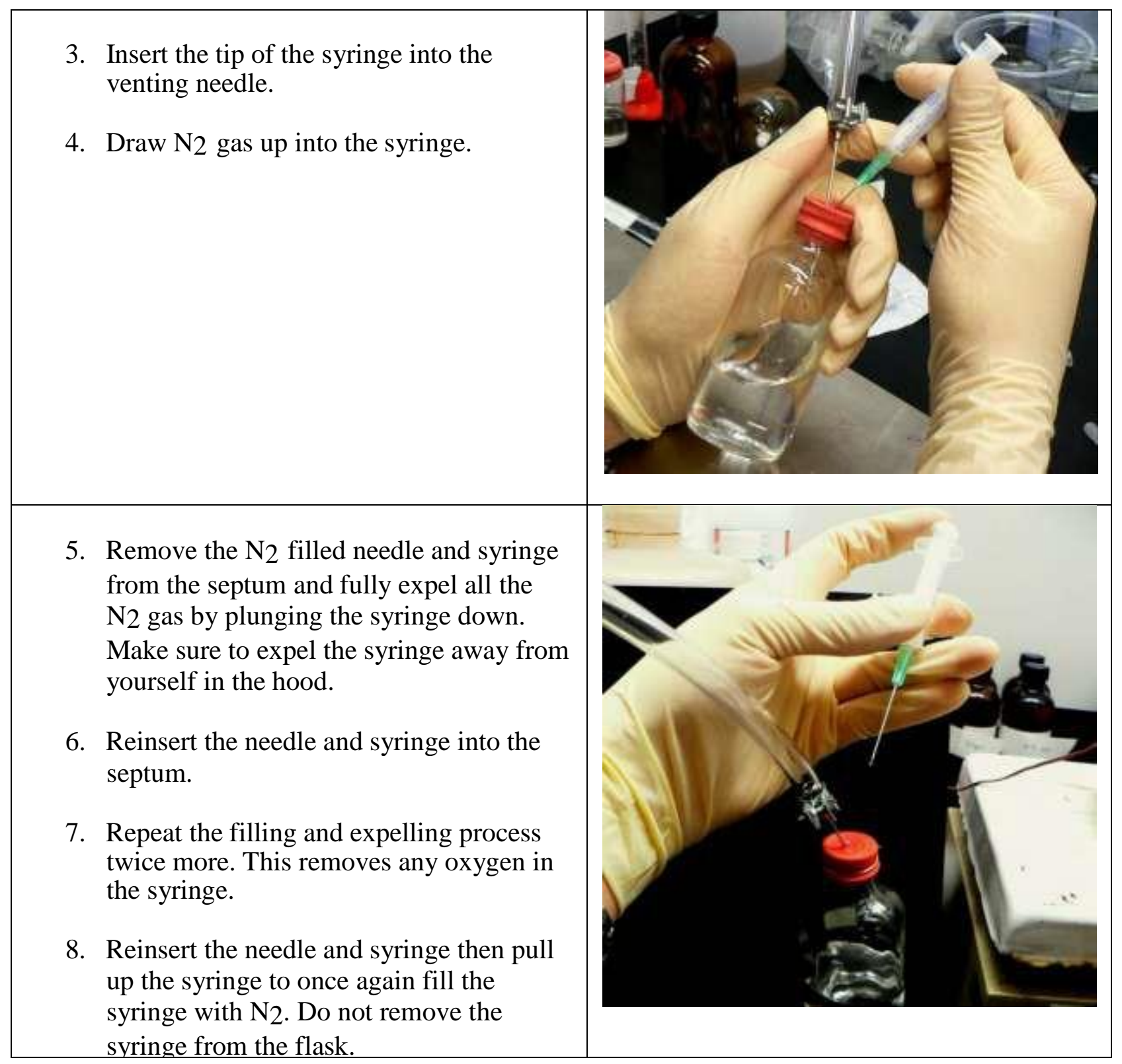




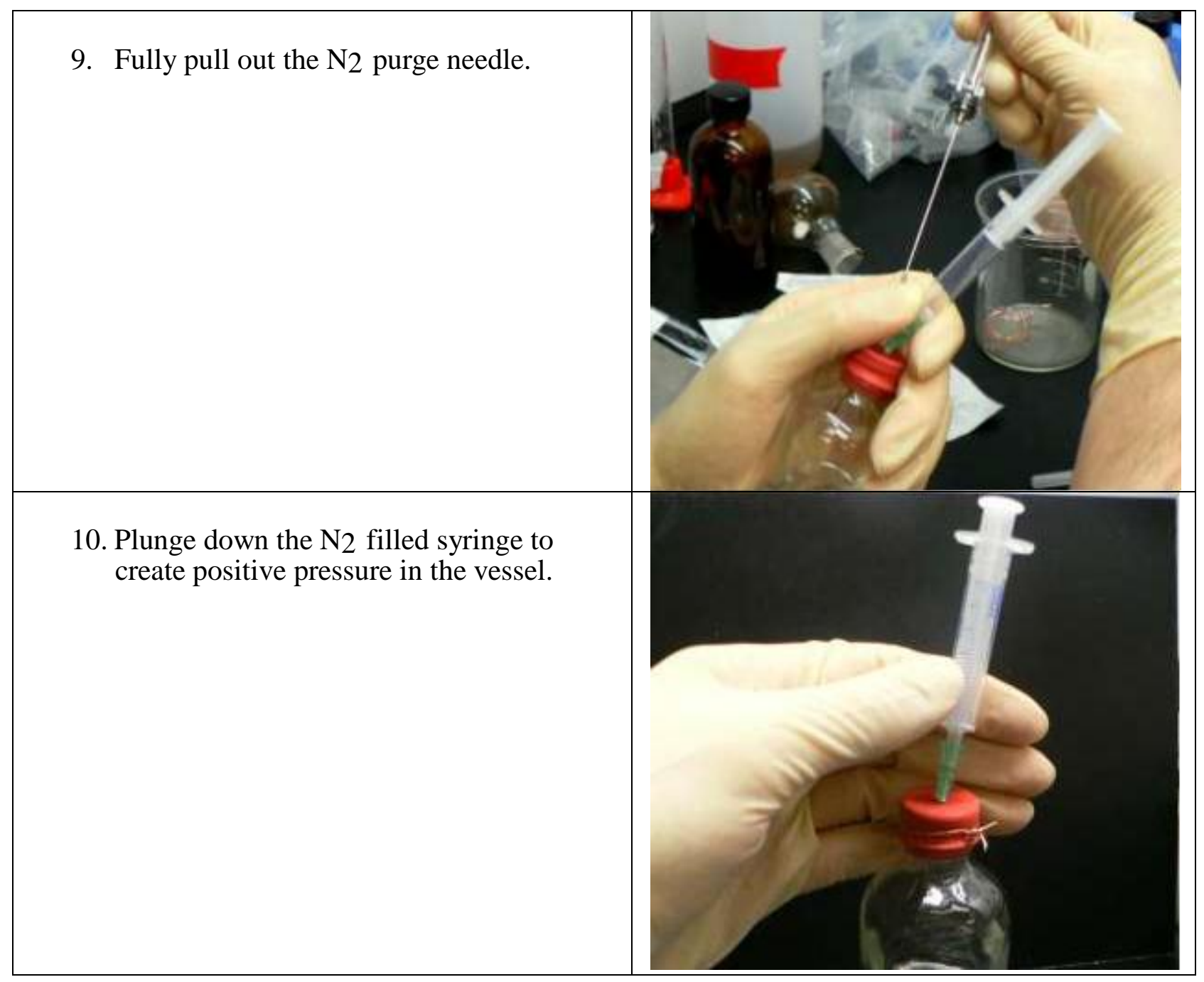


11. Invert the chemical vessel and syringe.

12. Slowly draw out the desired amount of solution into the syringe. Make sure to keep the needle tip in the liquid to prevent gas from entering the syringe.

13. Quickly and carefully insert the needle into the center of the septum of the desired vessel and slowly plunge down until all the liquid is expelled.

14. If more of the purged chemical is needed, quickly reinsert the syringe into the purged solution and draw out more chemical.

15. Chemicals must be repurged if left for longer than 10 minutes.

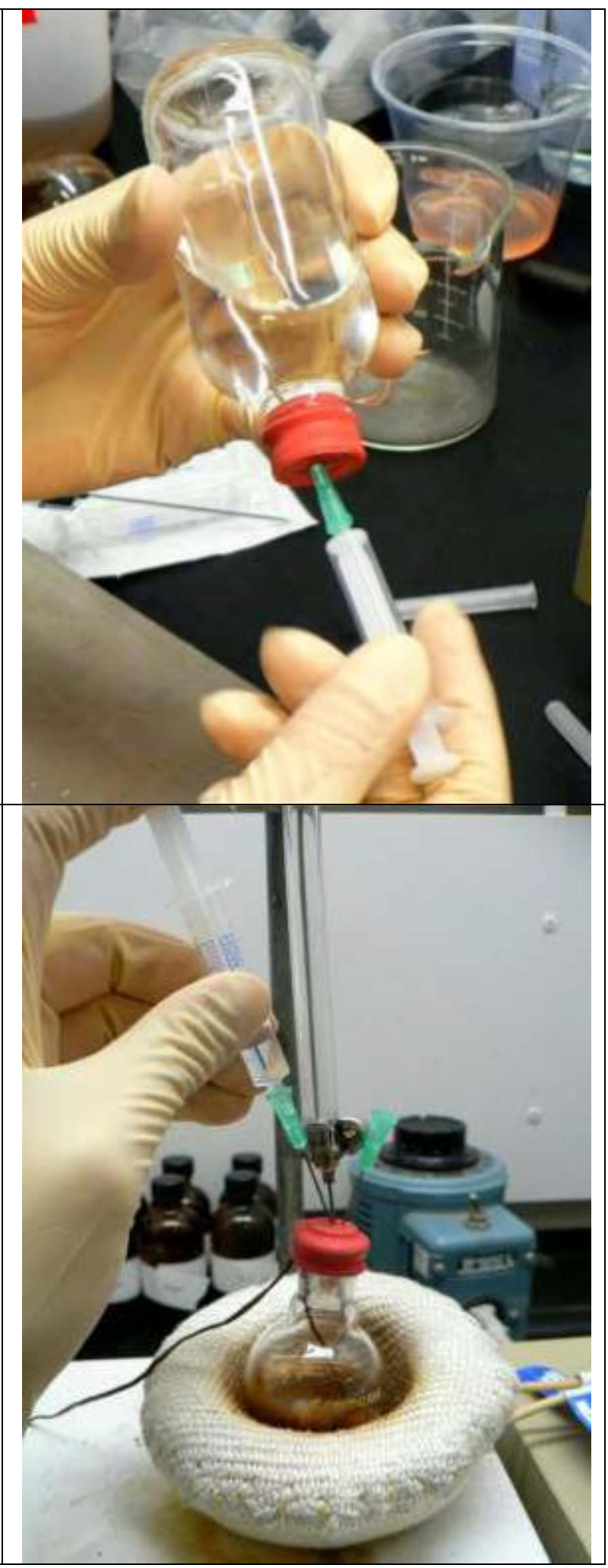




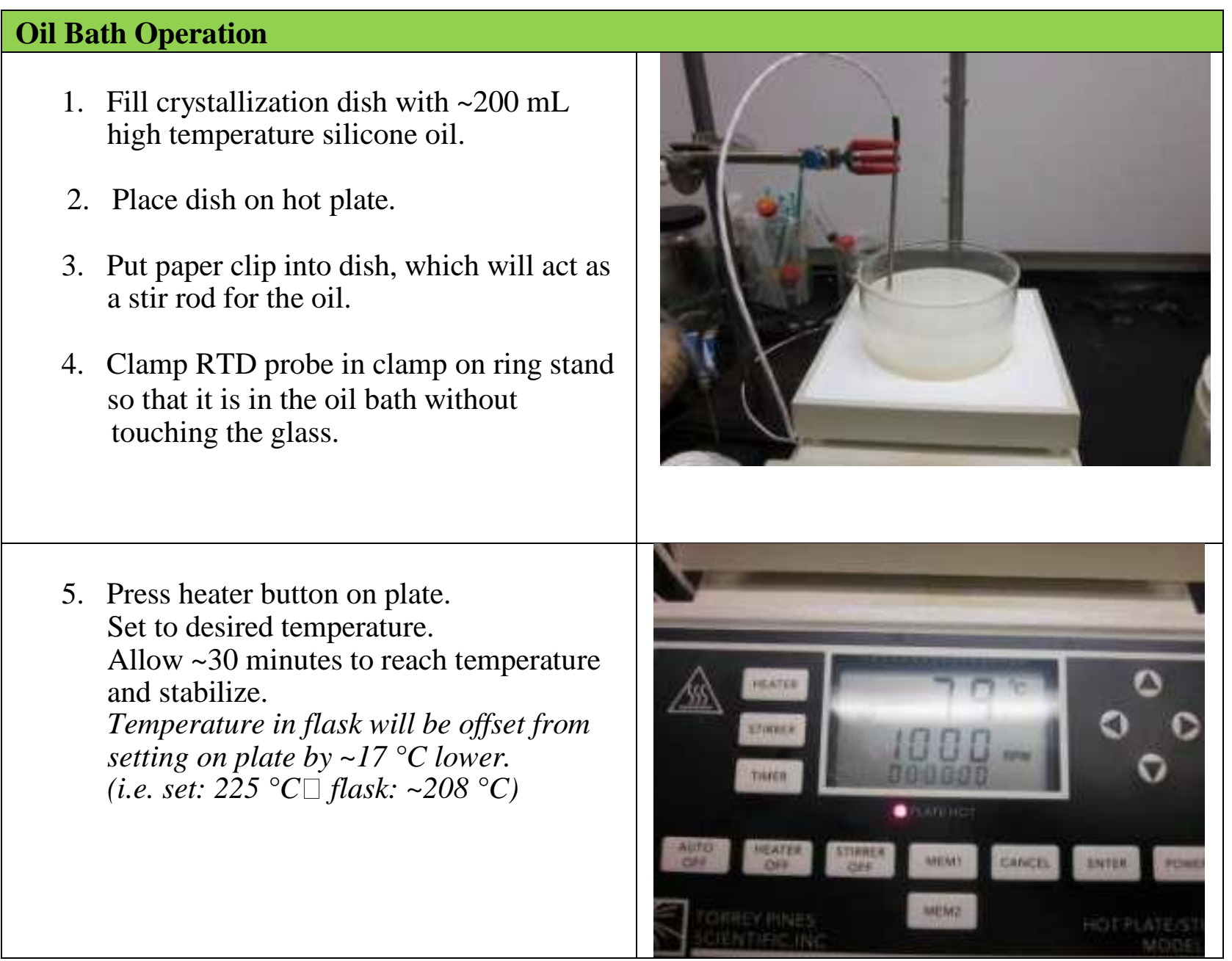


6. Put stir bar into reaction flask. Put thermocouple into the flask at this point if necessary, sealing it between the glass and the septum.

7. Put septum onto flask to seal it.

8. Clamp reaction flask in 3-finger clamp.

9. Lower the reaction flask into the oil bath. Make sure the flask is not touching the bottom of the dish.

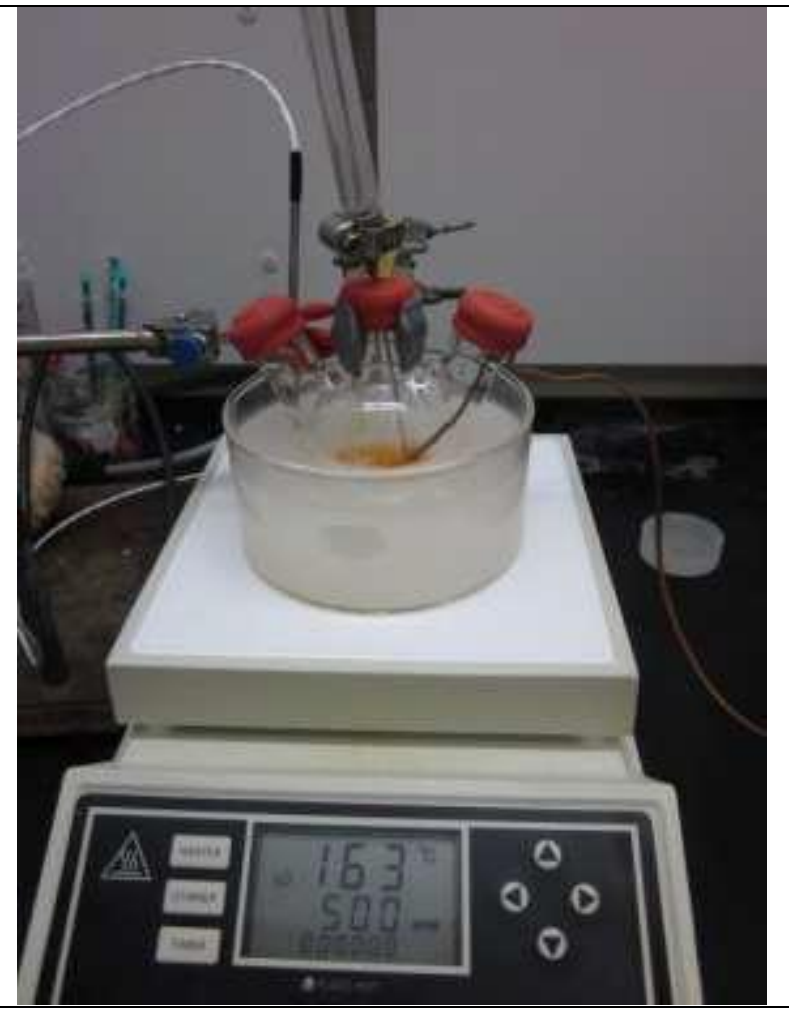

10. Press stirrer button on plate. Set to desired stir speed.

11. When finished with reaction, turn off heater and stirrer.

12. Raise clamped flask out of oil bath and clean oil off with kimwipe with toluene and then acetone.

13. Allow oil bath to come to room temperature.

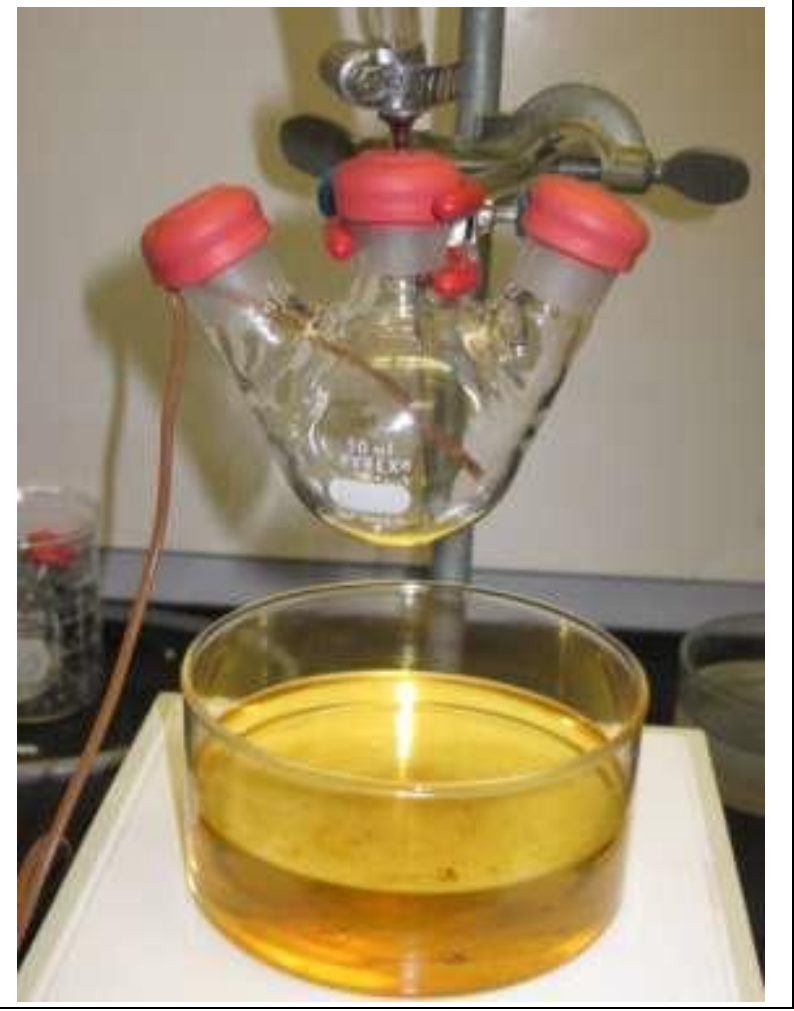




\section{Se-TOP Procedure}

1. Purge Octadecene (ODE) following the Purging Procedure for $\sim 15$ minutes.

2. Weigh out $33 \mathrm{mg}$ Se powder into a 50 $\mathrm{mL}$ round bottom flash. Make sure the flask is clean and dry.

3. Drop a small stir bar in the flask. Cap flask with a rubber septum.
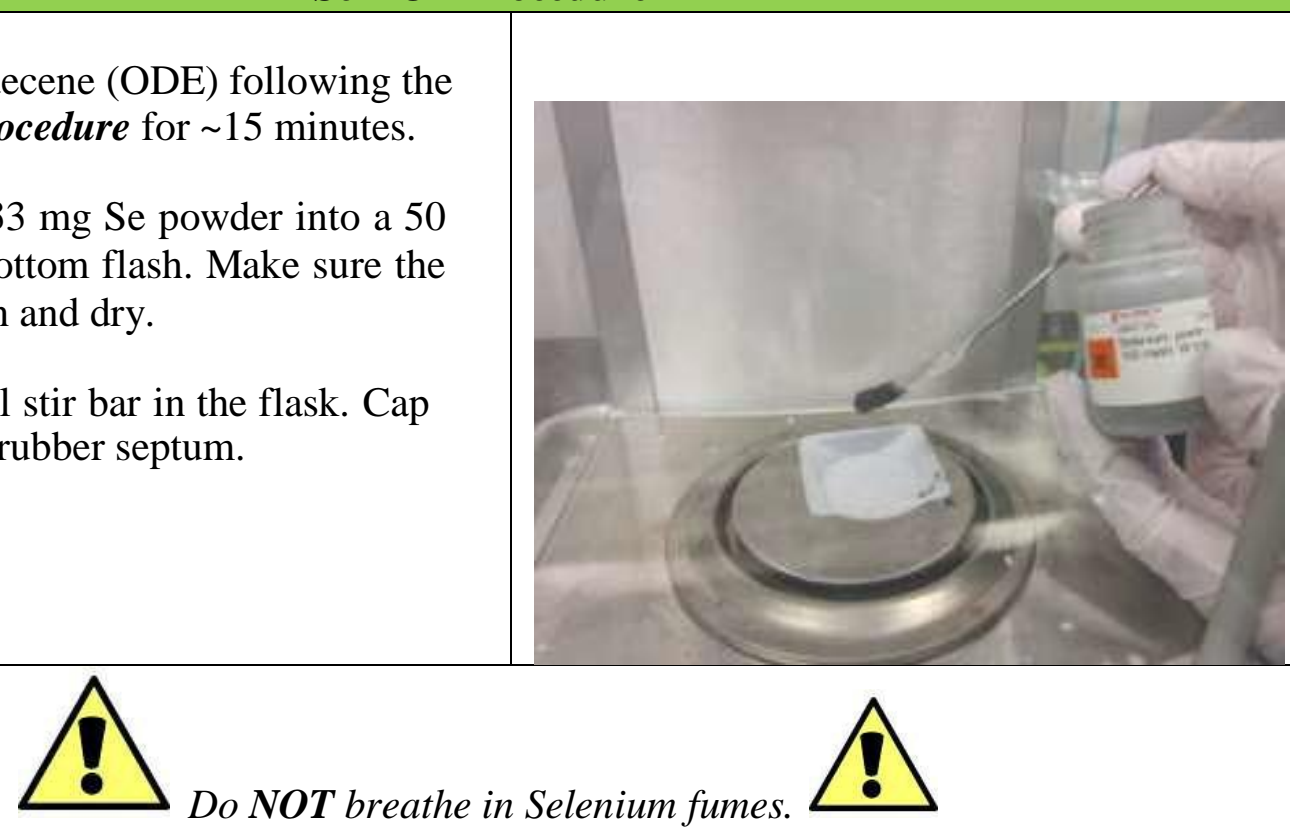

4. Clamp the neck of the flask in a 3-finger clamp on ring stand.

5. Set heat to $150{ }^{\circ} \mathrm{C}$.

6. Purge the flask with $\mathrm{N}_{2}$ gas by inserting the purge needle followed by a venting needle. Allow to purge for 10 minutes.

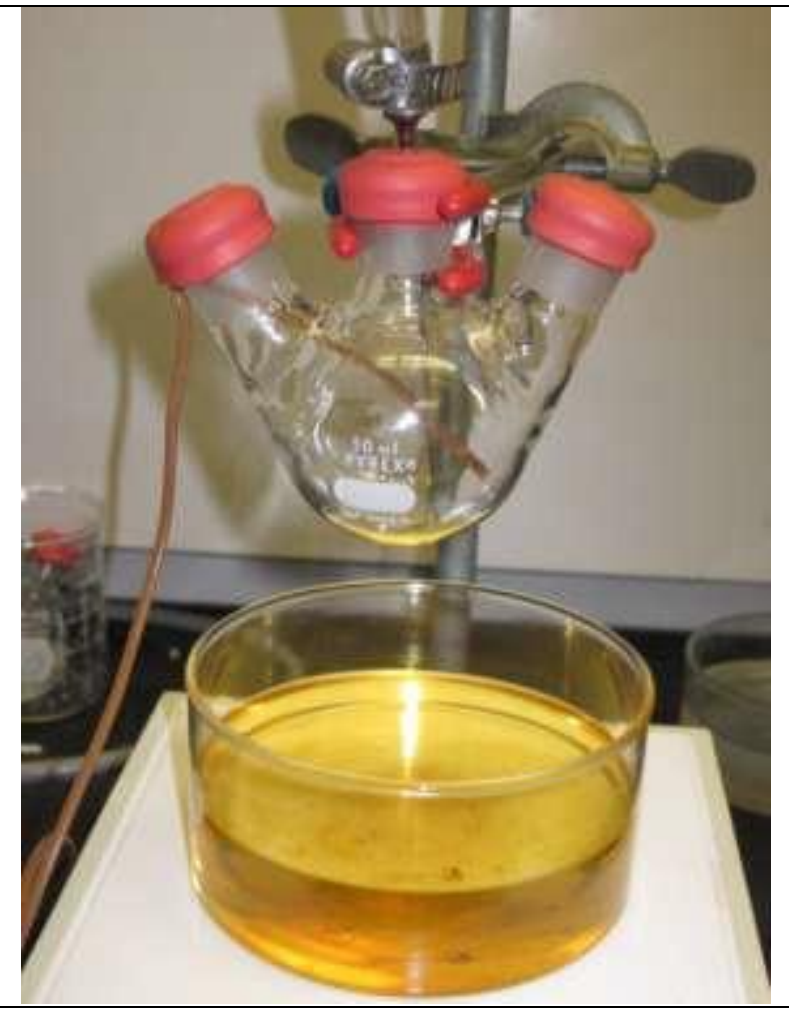


7. Transfer $5 \mathrm{~mL}$ ODE into the flask using the Syringing Procedure.

8. Lower flask into oil bath.

9. Set stir to $500 \mathrm{rpm}$.

10. Begin purging trioctylphosphine using the Purging Procedure.

Purge for $\sim 15$ minutes.

11. Using the Syringing Procedure, add $0.4 \mathrm{~mL}$ of purged TOP to the Se-TOP reaction flask. The majority of the selenium should dissolve immediately.

12. Continue stirring at $150{ }^{\circ} \mathrm{C}$ until the solution is completely clear. If all the selenium does not dissolve, it may be a sign that oxygen was introduced into the reaction and oxidize the TOP before the selenium and TOP can react.

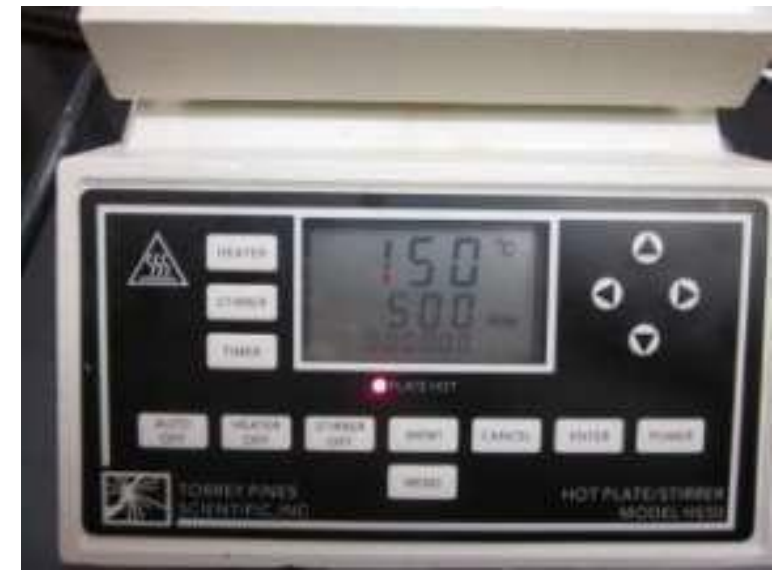

13. Remove the solution from the oil bath and pull out the $\mathrm{N}_{2}$ needle and vent needle.

14. Once it has cooled, clean the outside of the flask with toluene and acetone. This SeTOP solution can be stored for up to a week and makes enough SeTOP precursor for five QD syntheses. 


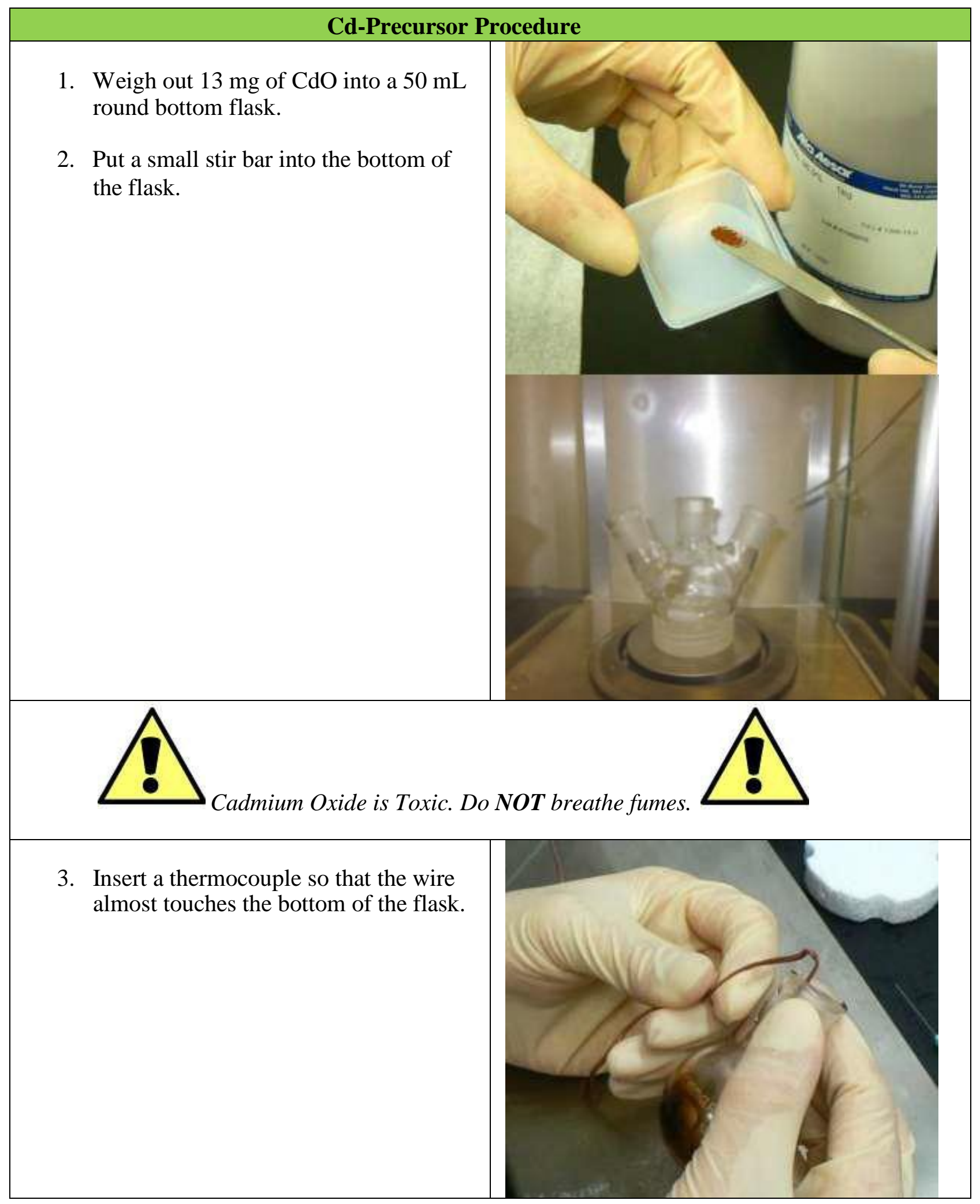




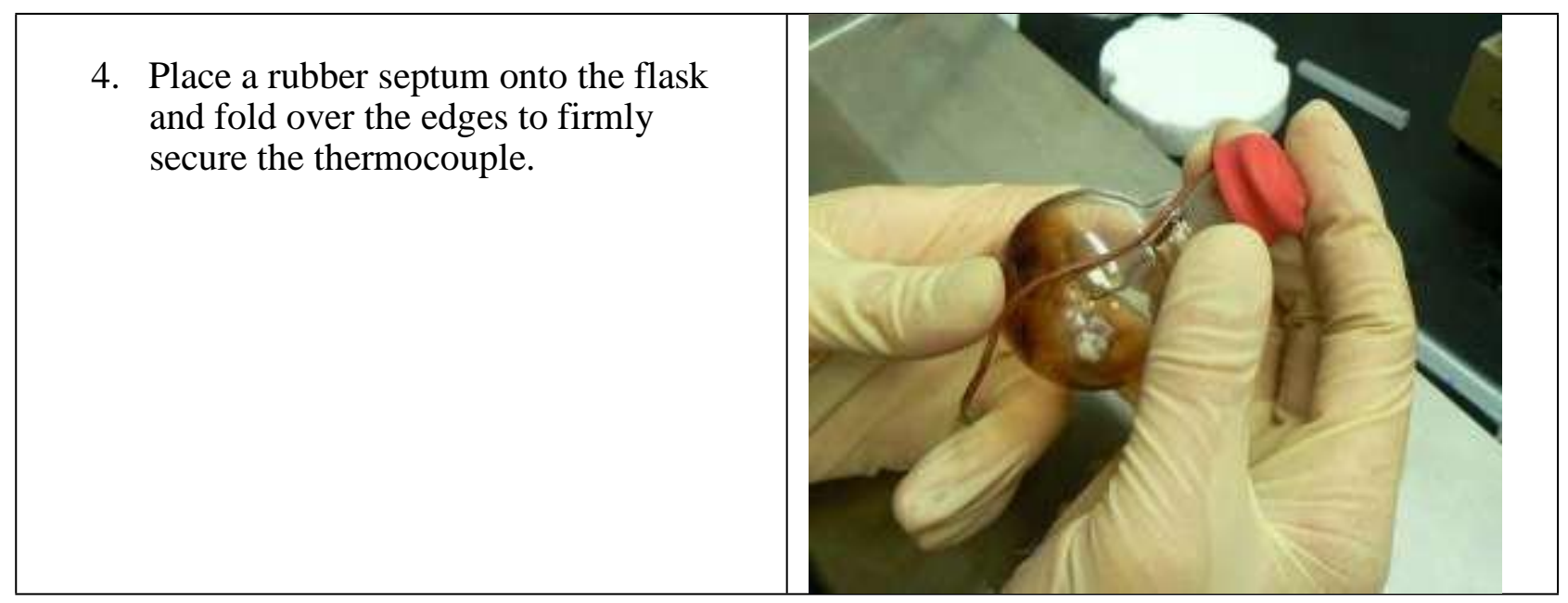




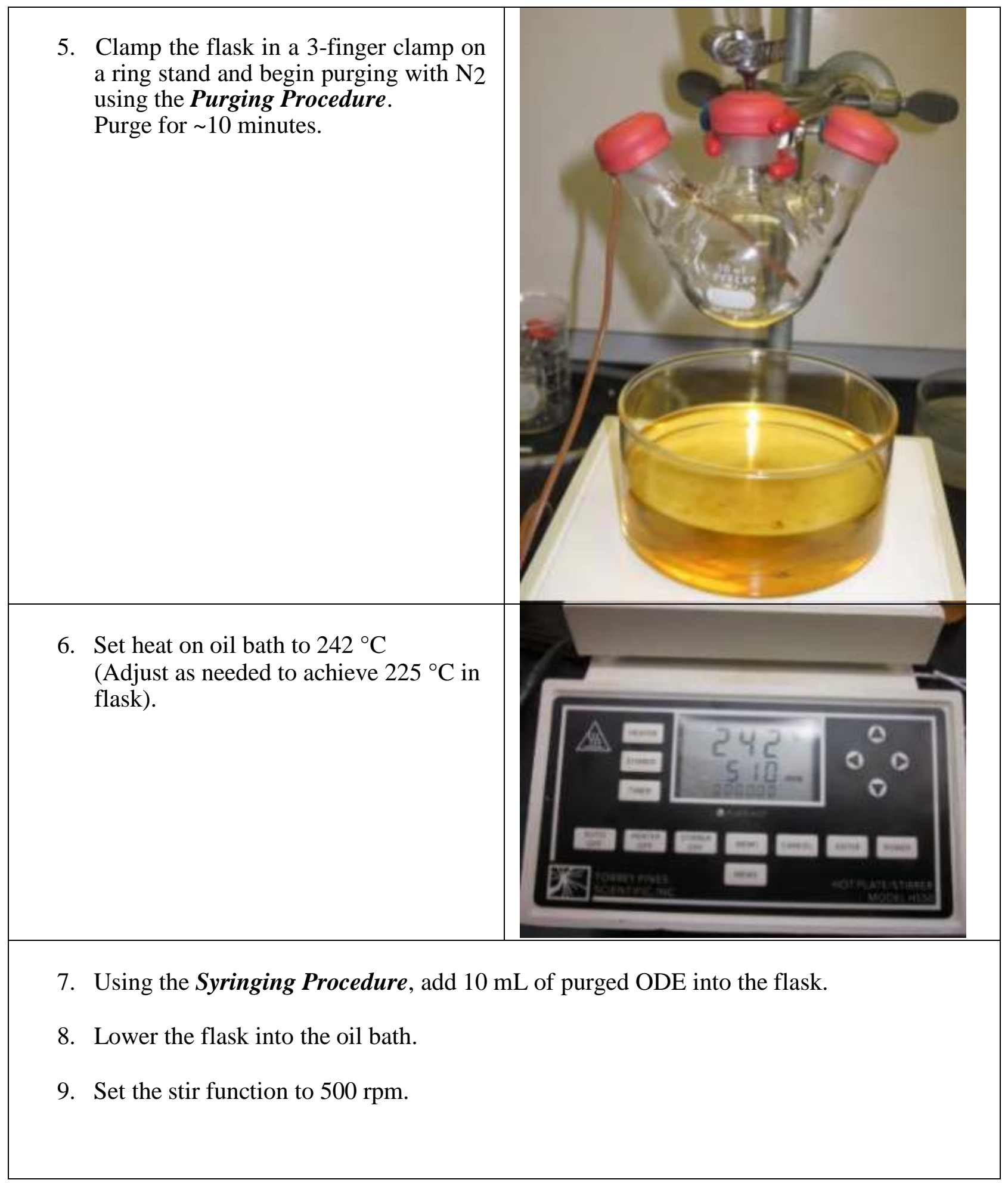


10. Purge the Oleic Acid using the Purging Procedure for $\sim 10$ minutes.

11. Using the Syringing Procedure, add $0.6 \mathrm{~mL}$ Oleic Acid to the Cd reaction flask.

12. Heat until solution becomes optically clear. $\mathrm{CdO}$ has a tendency to stick to the walls of the flask, so the flask should be agitated occasionally to prevent build up on walls.

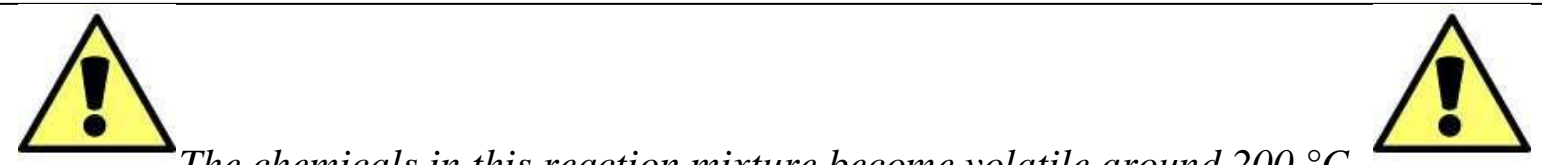

The chemicals in this reaction mixture become volatile around $200{ }^{\circ} \mathrm{C}$. Do NOT breathe fumes. 


\section{Sample Removal}

Caution: This process requires safely handling high-temperature organic, volatile chemicals. Two people must be present and all those present must wear appropriate safety equipment at all times.

Prepare syringes and vials while the $\mathrm{Cd}$ precursor is heating.

1. Attach one metal needle tip securely to a plastic $3 \mathrm{~mL}$ syringe and one to a glass $5 \mathrm{~mL}$ syringe with Luer lock.

2. Remove the caps from clean, dry vials.

3. Once the Cd precursor becomes optically clear, ensure that its temperature is stable at $225^{\circ} \mathrm{C}$ (adjust hot plate setting as needed to achieve stability at $225^{\circ} \mathrm{C}$ ).

4. Once stable at $225 \mathrm{C}$, quickly inject 1 $\mathrm{mL}$ of room-temperature Se-TOP precursor into the hot $\mathrm{Cd}$ precursor.

Start timing when the SeTOP precursor is injected.

A white fog will immediately form and the solution will begin changing color to a pale yellow.

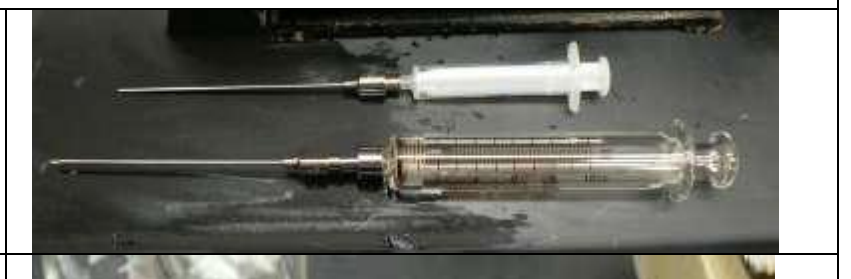




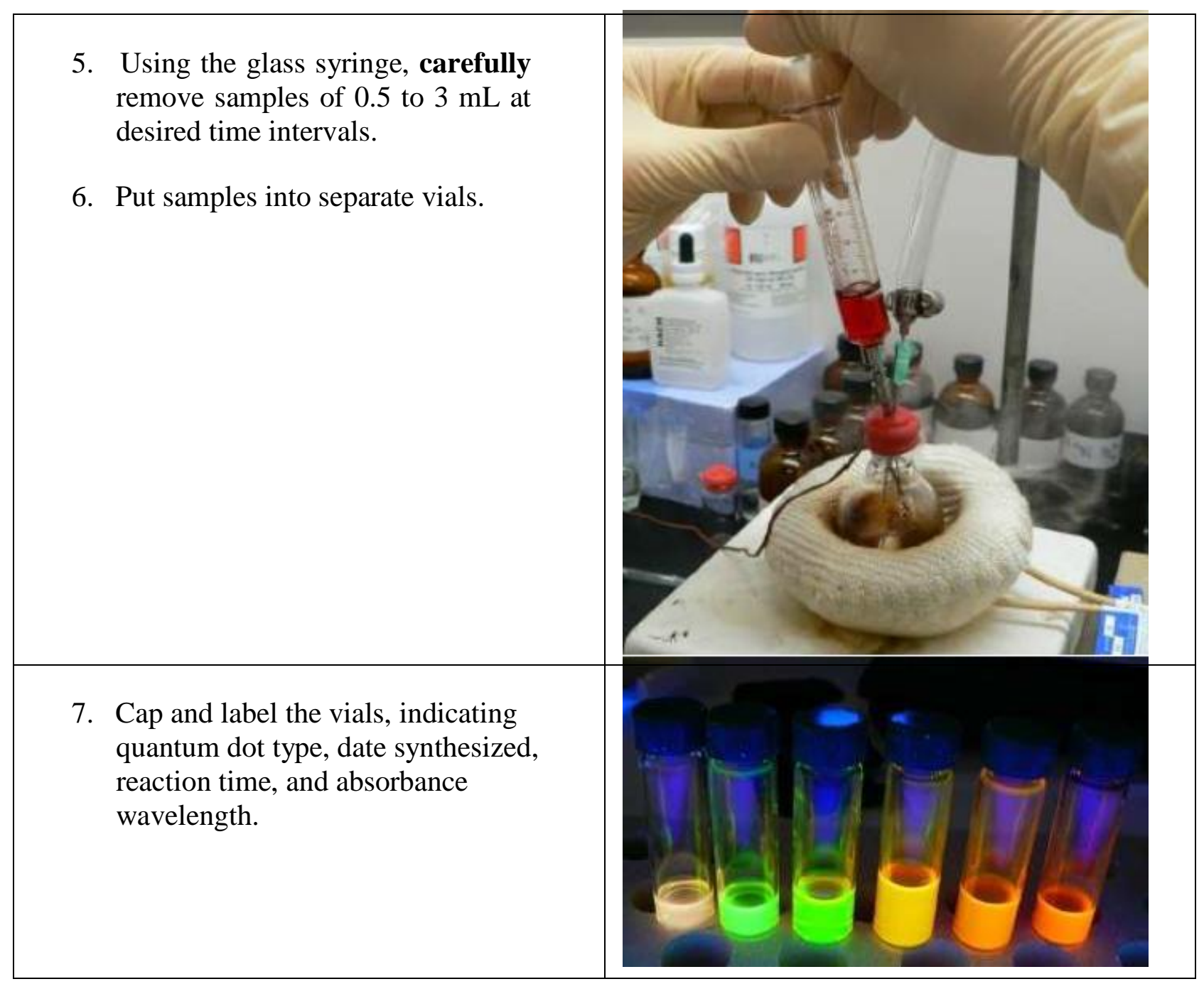




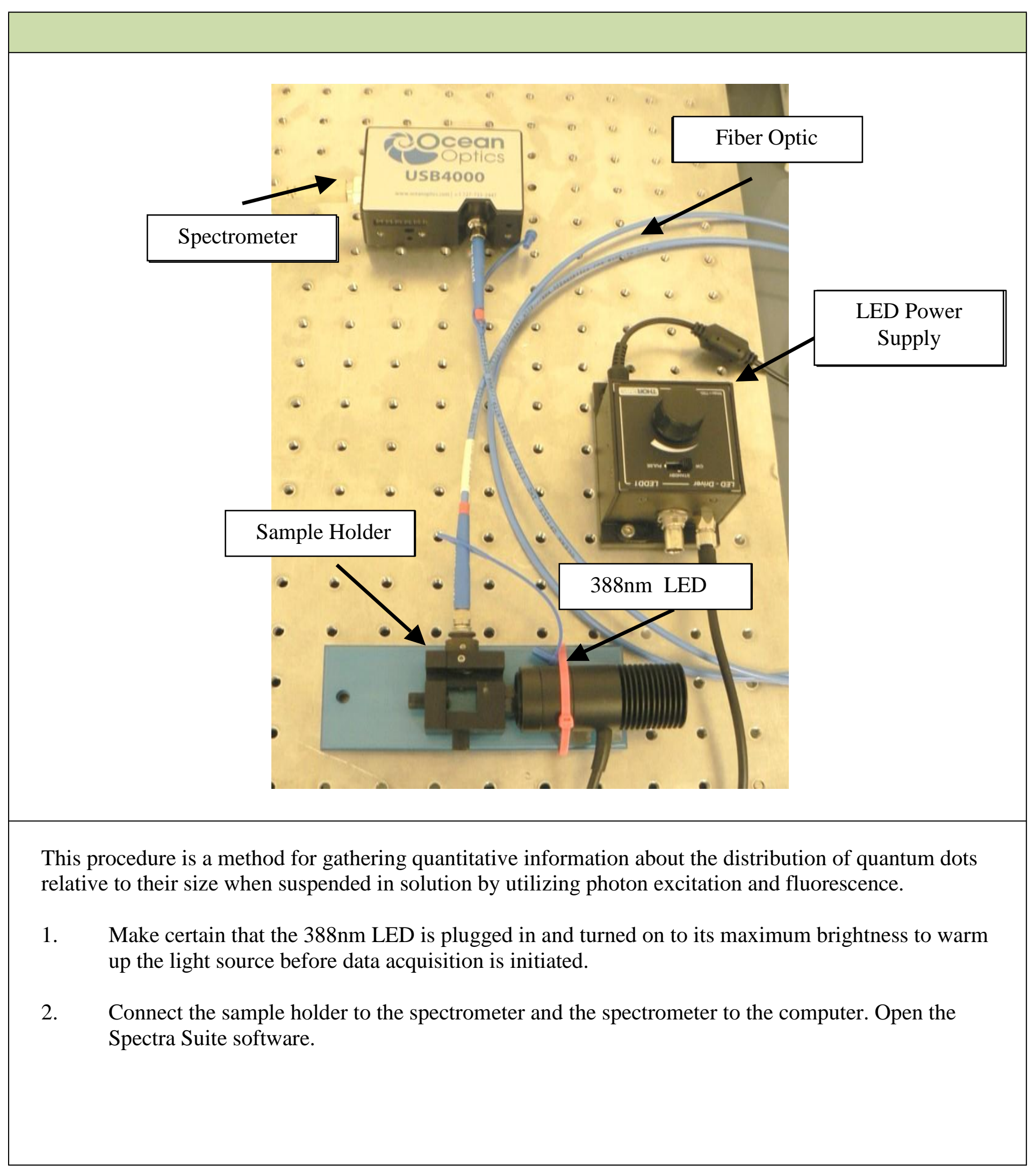




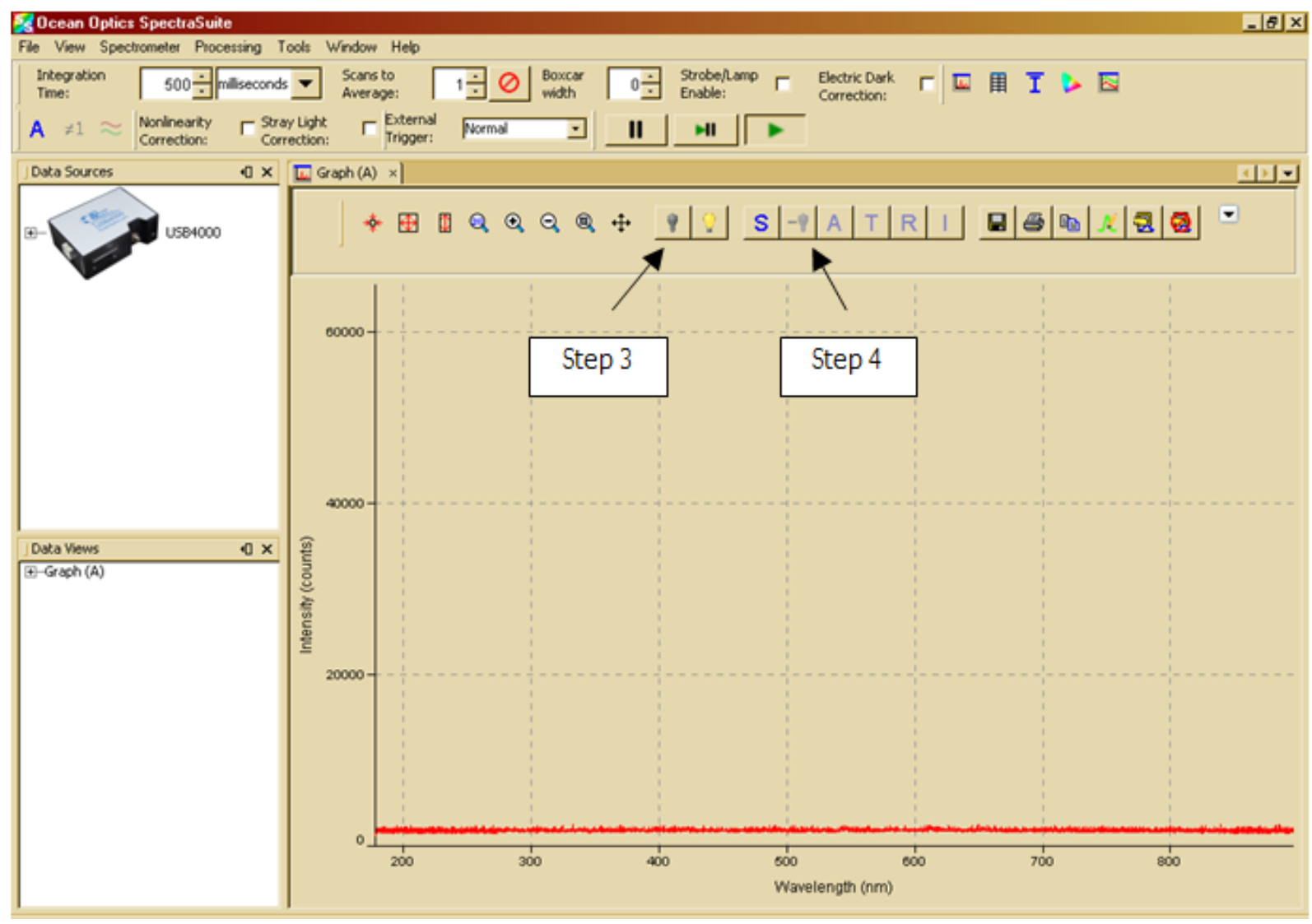

3. With the LED on, but the sample holder empty, take a dark spectrum reading by clicking on the unlit light bulb icon.

4. Click on the icon containing both a "-“" and an unlit light bulb. This subtracts the background noise preventing its influence on the $\mathrm{QD}$ readings.

5. Place a minimum of $2 \mathrm{~mL}$ of the QD solution to be tested into a clean cuvette. Place the cuvette inside the cuvette holder making certain to secure it in place.

6. Click on the icon of two line pieces of paper to copy the resulting fluorescence spectra onto the clip board, then transfer this data onto an Excel spread sheet for further analysis.

7. Using the cursor, click on the fluorescence peak at its maximum value; this is your center max wavelength (CMW). Divide the intensity value associated with the CMW by two. Find the wavelength values on each side of the peak associated with this new intensity value. The difference between the two wavelength values is the full width at half $\max$ (FWHM). 


\section{B.2 - Absorbance Measurement Procedure}

\section{Materials:}

- Spectra Suite Software

- Ocean Optics USB 4000 UV-Vis spectrometer

- DH 2000 BAL light source: combines the output of deuterium and tungsten halogen sources into a single optical path from $215-2000 \mathrm{~nm}$.

- Quartz cuvette $-1 \mathrm{~cm}$ path length (glass or disposable plastic cuvettes may also be used)

- Two 400 micron optical fibers

- Sample holder

*When not actively taking measurements turn the toggle switch on the light source to the close position to prevent inadvertent exposure to the light source.

\section{Procedure:}

1. Use two 400 micron optical fibers at $180^{\circ}$ relative to one another, one to transmit light from the source to the sample and one to transmit light from the sample to the spectrometer.

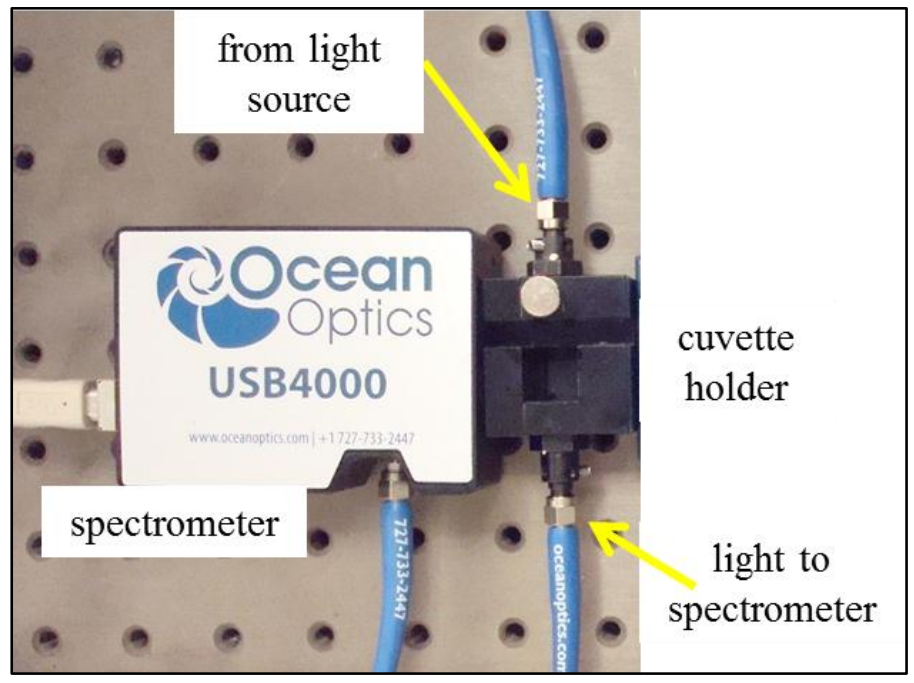


2. Load the Spectrsuite software, and then turn on the light source by flipping the power switch at the back.

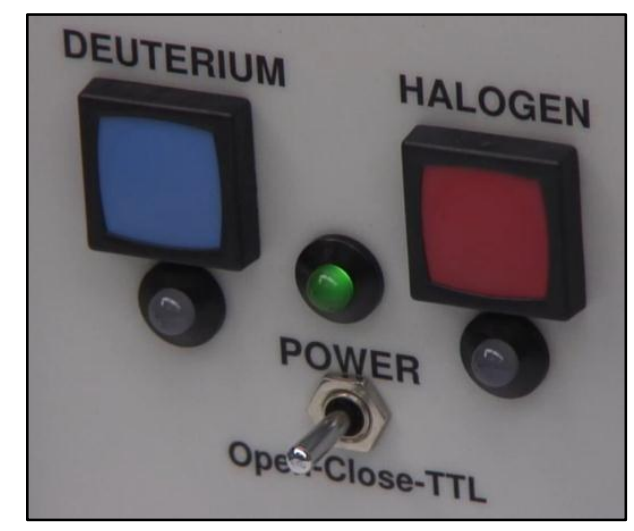

3. Press the blue button to turn on the deuterium source.

The light will blink momentarily while the source is warming up. When the light stops blinking turn on the tungsten halogen source by pressing the red button. Allow the light source 20 to 30 minutes to completely warm up.

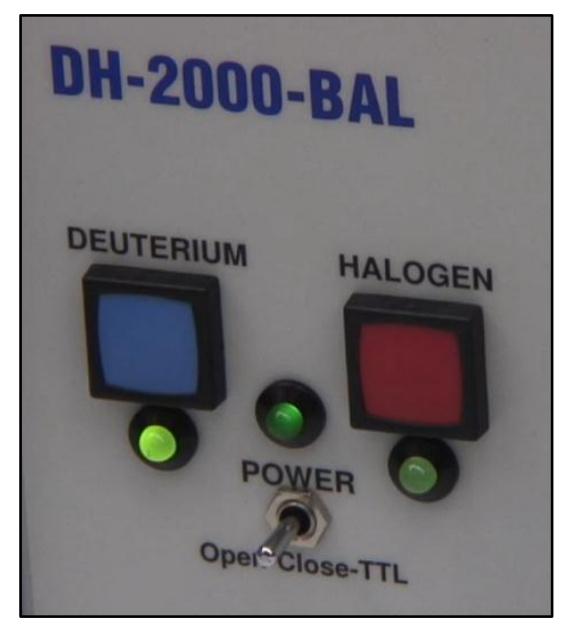

4. Moving the toggle switch to the open position will open the magnetic shutter and allow the light to move through the optic fiber and interact with the sample. 
5. To create a reference spectrum insert a cuvette with $3 \mathrm{~mL}$ of the solvent into the sample holder. When using CdSe/ZnS QDs synthesized using the standard protocol, the reference solvent will be ODE.

6. Open a new "Absorbance" file.

7. Move the toggle switch to the open position.

8. Click on the yellow light bulb icon in the control panel. This will store the reference spectrum.

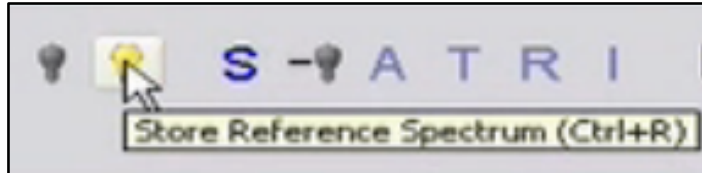

9. Move the toggle switch to the closed position.

10. Click on the grey light bulb icon in the control panel. This will store the dark spectrum. The processing mode icons will now turn dark blue indicating that they are active.

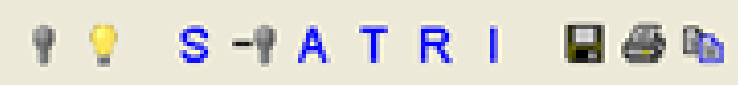

11. Click on the "A" icon in the control panel.

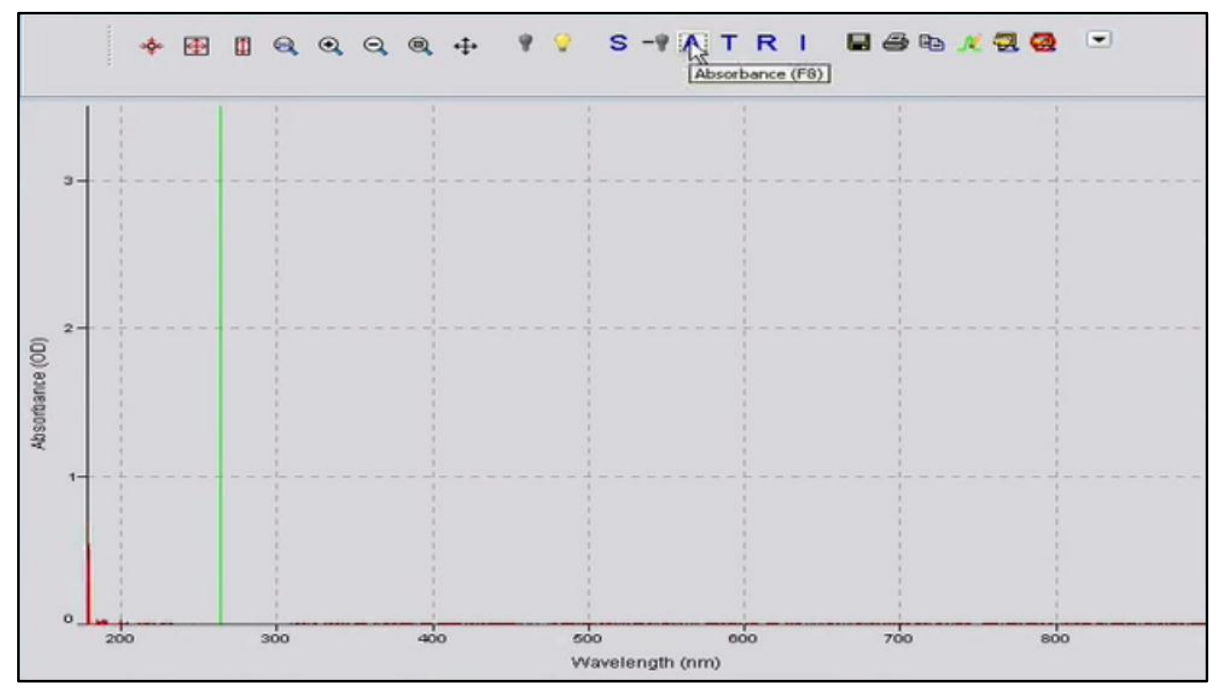

166 
Once the absorbance processing mode has been initiated, a spectrum similar to the one shown above should appear, indicating an absorbance measurement of zero.

12. Remove the reference sample cuvette of solvent.

13. Place in the test sample cuvette.

14. Set the integration time to a value where the entire spectrum can be seen. For ODE this is approximately $5 \mathrm{~ms}$.

\begin{tabular}{|c|c|c|c|c|c|c|c|c|c|}
\hline \multicolumn{10}{|c|}{ Es Ocean Optics SpectraSuite } \\
\hline \multirow[t]{2}{*}{ File } & \multicolumn{2}{|c|}{ New Spectrometer } & \multicolumn{2}{|c|}{ Processing } & Window & \multicolumn{2}{|l|}{ Help } & \multirow[b]{2}{*}{$\begin{array}{l}\text { Eoxcar } \\
\text { wideh }\end{array}$} & \multirow[b]{2}{*}{$2 \hat{F}$} \\
\hline & $\begin{array}{l}\text { Integration } \\
\text { Time: }\end{array}$ & & $35 \hat{\mathrm{Z}}$ & milliseconds & $\sim$ & $\begin{array}{l}\text { Scans to } \\
\text { Average: }\end{array}$ & $10 \hat{\sim}$ & & \\
\hline
\end{tabular}

15. Set the scanning average value to 10 . This reduces the signal to noise ratio.

16. Set the box car value to 2 . This smooths out the signal.

The absorbance spectrum for ODE should appear to be similar to the spectrum shown below.

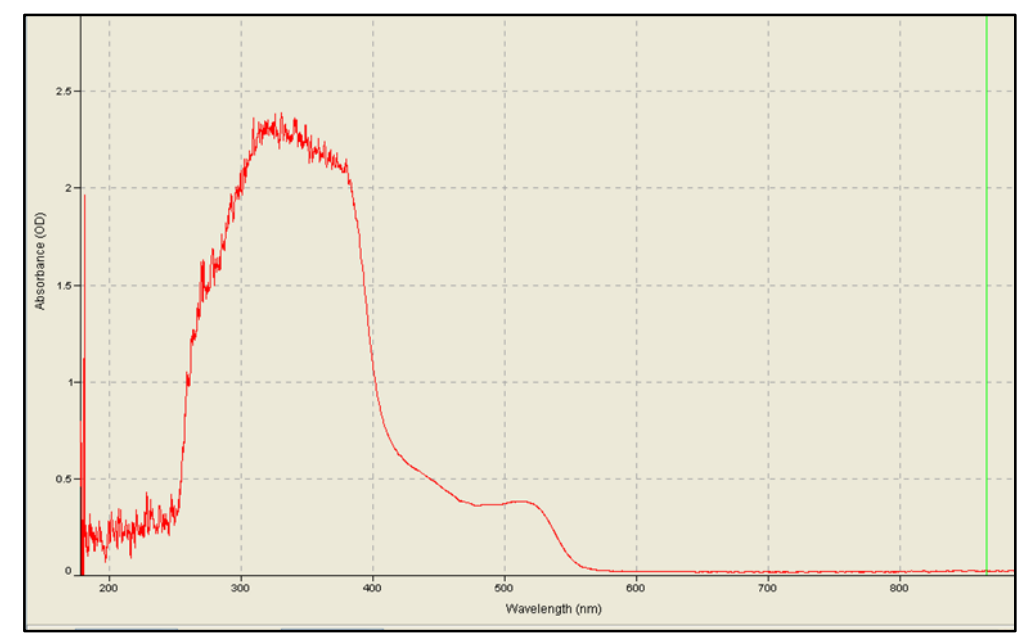

\section{$\underline{\text { Additional Information }}$}

For additional methods of acquiring absorbance measurements refer to the tutorial provided on the Ocean Optics website at http://www.oceanoptics.com/videos/absorbance.htm. 


\section{Appendix B.3 - Wavelength Specific CdSe and CdSe/Zn Synthesis}

All of the following synthesis directions are given with the assumption that the standard

$\mathrm{CdSe} / \mathrm{ZnS}$ synthesis procedure given in Appendix B.1 has been read and understood.

\section{Procedures:}

White Fluorescing CdSe Cores

1. Use single batch amounts of $\mathrm{Cd}$ and Se-TOP precursor, 10 and $1 \mathrm{~mL}$ respectively.

2. Heat $\mathrm{Cd}$ precursor to a minimum of $215^{\circ} \mathrm{C}$ and add $1 \mathrm{~mL}$ of the Se-TOP precursor.

3. Extract a $5 \mathrm{~mL}$ sample no more than 15 seconds after injection of the Se-TOP precursor and deposit the sample into a $15 \mathrm{~mL}$ glass vial.

*Temperatures greater than $215^{\circ} \mathrm{C}$ can be used.

$\underline{545 \pm 5 \mathrm{~nm} \text { CdSe Cores }}$

1. Use single batch amounts of $\mathrm{Cd}$ and Se-TOP precursor, 10 and $1 \mathrm{~mL}$ respectively.

2. Heat $\mathrm{Cd}$ precursor to $215^{\circ} \mathrm{C}$ and add $1 \mathrm{~mL}$ of the Se-TOP precursor.

3. Extract a $5 \mathrm{~mL}$ sample 60 seconds after injection of the Se-TOP precursor and deposit the sample into a $15 \mathrm{~mL}$ glass vial.

\section{$\underline{555 \pm 5 \mathrm{~nm} \text { CdSe Cores }}$}

1. Use single batch amounts of $\mathrm{Cd}$ and Se-TOP precursor, 10 and $1 \mathrm{~mL}$ respectively.

2. Heat $\mathrm{Cd}$ precursor to $225^{\circ} \mathrm{C}$ and add $1 \mathrm{~mL}$ of the Se-TOP precursor.

3. Extract a $5 \mathrm{~mL}$ sample 60 seconds after injection of the Se-TOP precursor and deposit the sample into a $15 \mathrm{~mL}$ glass vial. 


\section{$\underline{495 \pm 5 \mathrm{~nm} \mathrm{CdSe} / \mathrm{ZnS} \text { QDs }}$}

1. Heat $5 \mathrm{~mL}$ of white fluorescing $\mathrm{CdSe}$ cores to $110^{\circ} \mathrm{C}$ for 10 minutes.

2. After 10 minutes of heating add $1 \mathrm{~mL} \mathrm{ZnS} \mathrm{precursor} \mathrm{drop-wise} \mathrm{over} \mathrm{a} \mathrm{period} \mathrm{of} 2$ minutes; heat for an additional 8 minutes for a total synthesis time of 20 minutes.

3. Remove the solution from the heat and when the internal temperature reaches $60^{\circ} \mathrm{C}$, quickly add $1 \mathrm{~mL}$ of butanol.

$\underline{540 \pm 5 \mathrm{~nm} \mathrm{CdSe} / \mathrm{ZnS} \text { QDs }}$

1. Heat $5 \mathrm{~mL}$ of white fluorescing $\mathrm{CdSe}$ cores to $160^{\circ} \mathrm{C}$ for 10 minutes.

2. After 10 minutes of heating add $1 \mathrm{~mL} \mathrm{ZnS} \mathrm{precursor} \mathrm{drop-wise} \mathrm{over} \mathrm{a} \mathrm{period} \mathrm{of} 2$ minutes; heat for an additional 8 minutes for a total synthesis time of 20 minutes.

3. Remove the solution from the heat and when the internal temperature reaches $60^{\circ} \mathrm{C}$, quickly add $1 \mathrm{~mL}$ of butanol.

$\underline{560 \pm 5 \mathrm{~nm} \mathrm{CdSe} / \mathrm{ZnS} \text { QDs }}$

1. Heat $5 \mathrm{~mL}$ of $550 \mathrm{~nm}$ CdSe cores to $110^{\circ} \mathrm{C}$ for 10 minutes.

2. After 10 minutes of heating add $1 \mathrm{~mL} \mathrm{ZnS} \mathrm{precursor} \mathrm{drop-wise} \mathrm{over} \mathrm{a} \mathrm{period} \mathrm{of} 2$ minutes; heat for an additional 8 minutes for a total synthesis time of 20 minutes.

3. Remove the solution from the heat and when the internal temperature reaches $60^{\circ} \mathrm{C}$, quickly add $1 \mathrm{~mL}$ of butanol.

$\underline{570 \pm 5 \mathrm{~nm} \mathrm{CdSe} / \mathrm{ZnS} \text { QDs }}$

1. Heat $5 \mathrm{~mL}$ of $550 \mathrm{~nm}$ CdSe cores to $160^{\circ} \mathrm{C}$ for 10 minutes.

2. After 10 minutes of heating add $1 \mathrm{~mL} \mathrm{ZnS} \mathrm{precursor} \mathrm{drop-wise} \mathrm{over} \mathrm{a} \mathrm{period} \mathrm{of} 2$ minutes; heat for an additional 8 minutes for a total synthesis time of 20 minutes. 
3. Remove the solution from the heat and when the internal temperature reaches $60^{\circ} \mathrm{C}$, quickly add $1 \mathrm{~mL}$ of butanol.

\section{Additional Information}

White fluorescing CdSe cores have an emission shift over time; therefore, if they are synthesized for later $\mathrm{ZnS}$ shelling they should be used within 10 days of synthesis. ODE can be added to CdSe/ZnS QDs with low intensities as a means of increasing the intensity but care should be taken to record and tract these alterations for future sample comparisons. 


\section{B.4 - Precipitation and Resuspension of CdSe/ZnS Quantum Dots}

\section{Materials:}

- Absolute ethanol

- Chloroform

- $2-4$ capped $15 \mathrm{~mL}$ centrifuge tubes

- Premiere Centrifuge XC-2000

- $3 \mathrm{~mL}$ syringe

- Vortex

- Metal reagent spatula

\section{Procedure:}

\section{$\underline{\text { Precipitation }}$}

1. Select CdSe/ZnS QD sample of interest suspended in ODE.

2. Divide the QD sample among an even number of centrifuge tubes with equal amounts of QD solution in each tube.

3. Add an amount of ethanol to each centrifuge tube that is equal to twice the amount of QD solution in each tube.

4. Cap and vigorously shake each tube.

5. Place all tubes, evenly distributed, in the centrifuge and spin at 4000 RPMs for 10 minutes.

6. Remove the supernatant. Begin removal with the syringe's needle at the surface of the supernatant to ensure the removal of the ODE.

7. Add an additional quantity of ethanol equal to the initial amount used in step 3 . Inject the ethanol quickly to promote agitation of the solution; if available vortex the solution. 
8. Repeat step 5 through 7 until a pellet of QDs have been securely precipitated into the tip of the centrifuge tube.

9. When an adequate precipitate is achieved, pour out the remaining supernatant.I

10. Place the centrifuge tubes, uncapped, in the fume hood for complete evaporation of any remaining supernatant.

$\underline{\text { Resuspension }}$

1. Select a centrifuge tube with a completely dry precipitate in the tip.

2. Using a metal reagent spatula, carefully remove any black precipitate that may be present.

3. Add an amount of chloroform to the precipitate equal to the initial volume of QD solution; i.e. if the initial solution in each centrifuge tube was $2.5 \mathrm{~mL}$, add $2.5 \mathrm{~mL}$ of chloroform.

4. Wait for QD precipitate to be completely suspended in chloroform.

Additional Information

If after addition of the chloroform the QDs continue to precipitate out of solution it may be necessary to perform the precipitation procedure a second time. Continual precipitation of the QDs out of the chloroform indicated that ethanol may still be present. 


\section{Appendix B.5 - Quantum Yield Calculation}

\section{Materials:}

- $1 \mathrm{mg}$ of fluorescein reference standard

- $6 \mathrm{~mL}$ of ethanol

- Sonicator

- $6 \mathrm{~mL}$ of octadecane (ODE)

- $9 \mathrm{~mL}$ of a CdSe/ZnS QD sample

- 10 cuvettes (disposable plastic cuvettes may be used)

- Cuvette tray

- $\mathrm{UV}-\mathrm{Vis}$ spectrometer

- Spectra Suite software

\section{Procedure:}

\section{$\underline{\text { Fluorescein Dilution }}$}

The optimal concentration is $20 \mu \mathrm{g} / \mathrm{mL}$ of fluorescein/ethanol. As a result of the limits of measurement inherent to many analytical scales, larger amounts of fluorescein may be measured out and the appropriated concentration arrived at through a series of dilutions. For instance, $1 \mathrm{mg}$ fluorescein diluted in $5 \mathrm{~mL}$ of ethanol yields a concentration of $200 \mu \mathrm{g} / \mathrm{mL}$. Further dilution can be achieved by taking $1 \mathrm{~mL}$ of this and adding to it $9 \mathrm{~mL}$ of ethanol. The resulting solution will have the desired concentration of $20 \mu \mathrm{g} / \mathrm{mL}$ of fluorescein/ethanol.

1. Put on gloves!

2. Weigh out $1 \mathrm{mg}$ of fluorescein on an analytical balance. Place in a $10 \mathrm{~mL}$ glass vial.

3. Add $5 \mathrm{~mL}$ of ethanol and securely cap the glass vial. 
4. Lower the solution into a sonicator for 30 seconds.

5. Remove $1 \mathrm{~mL}$ of the fluorescein/ethanol solution and place it in a $15 \mathrm{~mL}$ glass vial.

6. Add $9 \mathrm{~mL}$ of ethanol and securely cap the glass vial.

7. Lower the solution into a sonicator for 30 seconds or until an even distribution is observed. This is your starting stock solution of fluorescein to be later diluted for the purpose of achieving a graphed linear line from which the slope will be used to calculate quantum yield. $\underline{\mathrm{CdSe} / \mathrm{ZnS} \text { Quantum Dot and Fluorescein Dilution }}$

Starting with a stock solution to be used as the $100 \%$ concentration standard from which all other dilutions will be made, 5 different concentrations, of both the QD sample and the fluorescein/ethanol sample, will be created: 100, 80, 60, 40, and $20 \%$.

1. Set aside $3 \mathrm{~mL}$ of the $9 \mathrm{~mL}$ stock QD solution, this will be used as your $100 \%$ concentration solution. Set $3 \mathrm{~mL}$ of this solution aside in a cuvette.

2. Take the other $6 \mathrm{~mL}$ of the QD sample and add $1.5 \mathrm{~mL}$ of ODE for a total of $7.5 \mathrm{~mL}$ of an $80 \%$ concentration. Set $3 \mathrm{~mL}$ of this solution aside in a cuvette to be used as your $80 \%$ concentration.

3. Take the other $4.5 \mathrm{~mL}$ of $80 \%$ solution and add $1.5 \mathrm{~mL}$ of ODE for a total of $6 \mathrm{~mL}$ of a $60 \%$ concentration. Set $3 \mathrm{~mL}$ of this solution aside in a cuvette to be used as your $60 \%$ concentration.

4. Take the other $3 \mathrm{~mL}$ of the $60 \%$ solution and add $1.5 \mathrm{~mL}$ of ODE for a total of $4.5 \mathrm{~mL}$ of a $40 \%$ concentration. Set $3 \mathrm{~mL}$ of this solution aside in a cuvette to be used as your $40 \%$ concentration. 
5. Take the other $1.5 \mathrm{~mL}$ of the $40 \%$ solution and add $1.5 \mathrm{~mL}$ of ODE for a total of $3 \mathrm{~mL}$ of a $20 \%$ concentration. An example of the progression of dilutions where $6 \mathrm{~mL}$ of $100 \%$ concentration $+1.5 \mathrm{~mL}$ of $\mathrm{ODE}=7.5 \mathrm{~mL}$ of $80 \%$ concentration is given in Table B.5.1.

Table B.5.1 Concentration and Dilution Calculation Table

\begin{tabular}{|c|c|c|c|c|}
\hline $\mathrm{QD}(\mathrm{mL})$ & $\%$ & $\mathrm{ODE}(\mathrm{mL})$ & $\mathrm{QD}(\mathrm{mL})$ & $\%$ \\
\hline 3 & 100 & 0 & 3 & 100 \\
\hline 6 & 100 & 1.5 & 7.5 & 80 \\
\hline 4.5 & 80 & 1.5 & 6 & 60 \\
\hline 3 & 60 & 1.5 & 4.5 & 40 \\
\hline 1.5 & 40 & 1.5 & 3 & 20 \\
\hline
\end{tabular}

6. Repeat this same series of dilutions starting with $6 \mathrm{~mL}$ of $100 \%$ concentration fluoresceing/ethanol, adding $1.5 \mathrm{~mL}$ of ethanol for a total of $7.5 \mathrm{~mL}$ of $80 \%$ concentration, etc.

\section{Excel Spreadsheet Calculation Using Fluorescence Spectrums}

1. For greater ease, line up the cuvettes containing each set of concentrations in the cuvette trays in order from 100 to $20 \%$ concentration.

2. Set the UV-Vis spectrometer to measure fluorescence.

3. Obtain a fluorescence spectrum for each of the ten samples and store the raw data in a wellorganized and labeled Excel spreadsheet.

4. Calculate the area under each fluorescence peak. This can be accomplished by using the sequence "=(SUMPRODUCT(C3:C6,B4:B7)-SUMPRODUCT(B3:B6,C4:C7)+B7*C7$\left.\mathrm{B} 3{ }^{*} \mathrm{C} 3\right) / 2$ " as it corresponds to the example data given in Table B.5.2. In this example the area under the peak would be 4100 (no units). 
Table B.5.2 Sample Data for Calculating the Area Under a Fluorescence Spectral Peak

\begin{tabular}{|c|c|c|}
\hline & $\begin{array}{c}\text { B } \\
\text { wavelength } \\
(\mathrm{nm})\end{array}$ & $\begin{array}{c}\text { C } \\
\text { intensity } \\
\text { (counts) }\end{array}$ \\
\hline 3 & 10 & 10 \\
\hline 4 & 20 & 100 \\
\hline 5 & 30 & 200 \\
\hline 6 & 40 & 100 \\
\hline 7 & 50 & 10 \\
\hline
\end{tabular}

$\underline{\text { Absorbance Spectrum Analysis }}$

1. Set the UV-Vis spectrometer to measure fluorescence.

2. Obtain an absorbance spectrum for each of the ten samples and store the raw data in a wellorganized and labeled Excel spreadsheet for later reference if necessary.

3. For each sample set pick one wavelength value, for example $388 \mathrm{~nm}$, and find the corresponding O.D. value on the y-axis as illustrated in Figure B.5.1. Use the same wavelength value for each spectrum within the set.

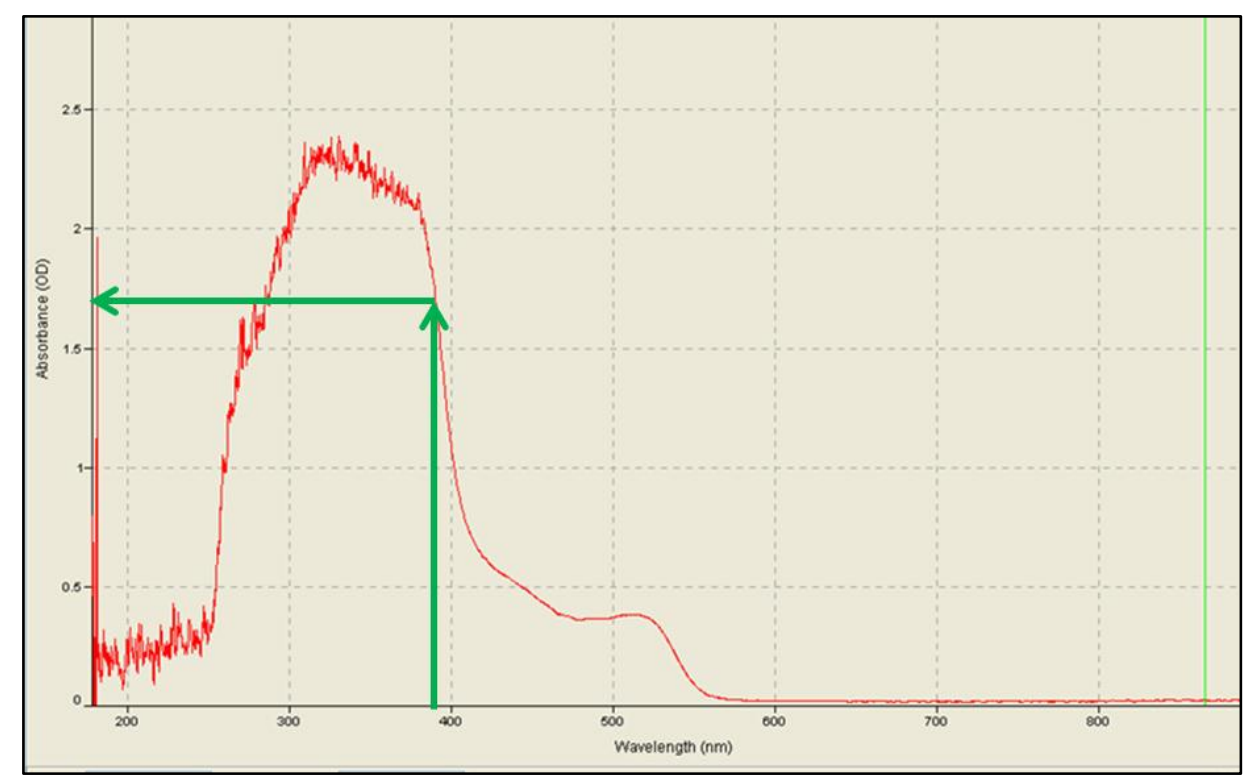

Figure B.5.1 Corresponding O.D. and wavelength values on an absorbance spectrum. 176 
4. Make a table in Excel of the paired values of each set for each concentration.

5. For each set of five concentrations, QD sample and fluorescein/ethanol sample, you will plot the fluorescence area value versus the O.D. absorbance value at each concentration. Do this for the QD data and for the fluorescein/ethanol data. Add a regression line with equation on each plot. The resulting equation will look something like $y=-4^{\mathrm{E} 07} \mathrm{x}+2.3566$ for the $\mathrm{QD}$ sample set and $y=3^{\mathrm{E} 07} \mathrm{x}-0.0596$ for the fluorescein/ethanol sample set. The value in front of the $x$, for example $-4^{\mathrm{E} 07}$, is your slope $m$ for the QD sample set and slope $m_{R}$ for the fluorescein/ethanol sample set.

\section{Quantum Yield Calculation}

Using the quantum yield value associated with the fluorescein reference standard as $Q_{R}$, the index of refraction of the QD solvent $n$, the index of refraction of the reference solvent $n_{R}$, and the corresponding QD and fluorescein slopes obtained from the previously plotted data, $m$ and $m_{R}$ respectively, use Equation B.5.1 to calculate the comparative quantum yield of the QD sample, $Q$.

$Q=Q_{R}\left(\frac{m}{m_{R}}\right)\left(\frac{n^{2}}{n_{R}^{2}}\right)$

Equation B.5.1

\section{$\underline{\text { Additional Information }}$}

When using CdSe/ZnS QD samples of less than 10mL, additional concentration and dilution scenarios can be easily calculated using the Dilution Calculator of Mass Concentration found at the website: http://endmemo.com/bio/dilution.php 
A less exact but preliminary quantum yield can be calculated for a single concentration, instead of the previous example using a series of five concentrations, using Equation B.5.2 where $I$ is the integrated fluorescence intensity of the fluorescence spectrum and $O D$ is the optical density value of the absorbance spectrum.

$Q=Q_{R}\left(\frac{I}{I_{R}}\right)\left(\frac{O D_{R}}{O D}\right)\left(\frac{n^{2}}{n_{R}^{2}}\right)$

Equation B.5.2 


\section{Appendix B.6 Solubilization and Phospholipid Encapsulation of CdSe/ZnS Quantum Dots}

The following is a standardized protocol for creating a solution of phospholipid (PL) encapsulated QDs (QD constructs) in an aqueous medium at a concentration of $10 \mathrm{mg} / \mathrm{mL}$ of phospholipids relative to the aqueous medium. The ratio of QDs to PLs and the concentration of PLs relative to the aqueous medium can be further adjusted to suit particular needs, however, it is recommended that a concentration of $10 \mathrm{mg} / \mathrm{mL}$ of PL/aqueous medium is not exceeded.

\section{Materials:}

- Fonterra 700 Phospholipid Mix

- Chloroform $\left(\mathrm{CHCl}_{3}\right)$

- Aqueous medium (AM)

- $50 \mathrm{~mL}$ glass beaker

- $15 \mathrm{~mL}$ glass vials (minimum of 2)

- Silicon oil heat bath

- Magnetic spin vane

- Analytic scale

- QDs precipitated from a $5 \mathrm{~mL}$ sample

\section{Procedure:}

1. Wear gloves!

2. Resuspend the QDs in chloroform using the procedure given in Appendix B.4. This will result in $5 \mathrm{~mL}$ of QDs suspended in chloroform.

3. Weigh out $100 \mathrm{mg}$ of phospholipids in a $15 \mathrm{~mL}$ glass vial.

4. Add $10 \mathrm{~mL}$ of chloroform to the vial containing the phospholipids. 
5. Mix the $\mathrm{QD} / \mathrm{CHCl}_{3}$ and phospholipids/ $\mathrm{CHCl}_{3}$ together in the $50 \mathrm{~mL}$ Beaker.

6. To maintain the initial concentration of PL, add an amount of AM equal to that of the chloroform used in the original suspension. In this case add $10 \mathrm{~mL}$ of the AM.

7. Set the magnetic stirrer to 200 RPM and place the beaker into the silicon oil bath after the bath has reached an equilibrium temperature of $55^{\circ} \mathrm{C}$. Position the beaker at an angle in the bath to promote agitation of the solution thereby increasing the rate of evaporation of the chloroform.

8. Monitor the solution for a change in consistency. The higher the concentration of PL the thicker the resulting solution will be.

9. Raise the beaker out of the silicon bath after 15 minutes and inspect for the presence of chloroform. When all chloroform appears to have been evaporated remove the beaker and place the solution into a clean $15 \mathrm{~mL}$ glass vial.

\section{$\underline{\text { Additional Information }}$}

Concentrations of PL/AM of $2.5 \mathrm{mg} / \mathrm{mL}$ using a 2:1 milliliter ratio of $\mathrm{PL} / \mathrm{CHCl}_{3}: \mathrm{QD} / \mathrm{CHCl}_{3}$ was seen to have the most consistent results in previous trials and should be further investigated for use in any biologic applications. 


\section{Appendix B.7 Introduction of QD Construct Test Solutions to Keratinocyte Cell Cultures}

\section{Materials:}

- Keratinocyte cells cultured on thin four-well slides

- QD construct test solutions

- Keratinocyte media

- Aspirator

- Incubator

- Optical microscope

- Digital camera or image capturing software and connected laptop

*All used with and aspirated off of the keratinocyte cells should be disposed of properly in a biohazard waste receptacle in accordance with Cal Poly guidelines and State law.

\section{Procedure:}

1. Place all test solutions in a water bath set to $37^{\circ} \mathrm{C}$ for at least 10 minutes before introducing them to the cell cultures.

2. Inspect cell cultures under an optical microscope to check confluency and to inspect for evidence of contamination. Cells should be at least $60 \%$ confluent with minimal contamination.

3. If the cells are deemed to be appropriately confluent, inspect each culture using the optical microscope; do this before removing any of the culture media. Note confluency, cell colony density, and cell shape throughout the culture as well as any abnormalities or unidentified entities that may be present. 
4. Take three images of each culture for an accurate and thorough representation of all cell and culture characteristics. *If using a digital camera, use the highest focus, no flash, and position the camera's lens just above the microscope eyepiece adjusting the aperture of the microscope as needed. Taking an initial picture of the four-well slide before commencing with taking images of the cells can aid in maintaining an accurate record.

4. Aspirate off the old culture media and add test solutions. Each round of trials should include at least one well, in every four-well set, that is kept as a control and therefore receives only fresh media.

5. Place the cells back into the appropriate incubator for the prescribed time point.

6. At the end of the experimental time point, remove the cells from the incubator and repeat steps 2 and 3, recording observations as well as taking images before removal of the test solutions.

7. Aspirate off the test solutions and rinse the cells twice with $1.5 \mathrm{~mL}$ of keratinocyte media leaving the second $1.5 \mathrm{~mL}$ of media in place. Repeat steps 2 and 3.

8. Using the procedures outlined in Appendices 8 and 9, fix and stain the cells for inspection under the confocal microscope.

Additional Information

The following are possible test solution and time point arrangements, previously described in Chapter 4, that can be used exactly as described or altered as needed:

Table 4.2 Summary of Round Two QD Construct Control and Test Solutions

\begin{tabular}{|c|c|c|c|c|c|}
\hline Well & Test Solution & Filter & $\begin{array}{c}\text { Stain } \\
\text { (a) }\end{array}$ & $\begin{array}{c}\text { Stain } \\
\text { (b) }\end{array}$ & $\begin{array}{c}\text { Stain } \\
\text { (c) }\end{array}$ \\
\hline a, b, c1.1 & Control - krt media & & $\mathrm{H} / \mathrm{H}$ & $\mathrm{L} / \mathrm{D}$ & $\mathrm{H} / \mathrm{H}$ \\
\hline $\mathrm{a}, \mathrm{b}, \mathrm{c} 1.2$ & 42612oEN.1a: PL/krt [10mg/mL] & $0.2 \mu \mathrm{m}$ & $\mathrm{H} / \mathrm{H}$ & $\mathrm{L} / \mathrm{D}$ & $\mathrm{H} / \mathrm{H}$ \\
\hline$a, b, c 1.3$ & {$[10 \mathrm{mg} / \mathrm{mL}]$} & $0.2 \mu \mathrm{m}$ & $\mathrm{H} / \mathrm{H}$ & $\mathrm{L} / \mathrm{D}$ & $\mathrm{H} / \mathrm{H}$ \\
\hline
\end{tabular}




\begin{tabular}{|l|l|l|l|l|l|}
\hline a, b, c1.4 & $52312 \mathrm{EN} 45.60 \mathrm{~b}: \mathrm{PL} / \mathrm{krt}[10 \mathrm{mg} / \mathrm{mL}]$ & $0.2 \mu \mathrm{m}$ & $\mathrm{H} / \mathrm{H}$ & $\mathrm{L} / \mathrm{D}$ & $\mathrm{H} / \mathrm{H}$ \\
\hline $\mathbf{a}, \mathbf{b}, \mathbf{c 2 . 5}$ & $42612 \mathrm{oEN} .1 \mathrm{a}: \mathrm{PL} / \mathrm{krt}[10 \mathrm{mg} / \mathrm{mL}]$ & $0.45 \mu \mathrm{m}$ & $\mathrm{L} / \mathrm{D}$ & $\mathrm{H} / \mathrm{H}$ & $\mathrm{L} / \mathrm{D}$ \\
\hline a, b, c2.6 & Control - krt media & & $\mathrm{L} / \mathrm{D}$ & $\mathrm{H} / \mathrm{H}$ & $\mathrm{L} / \mathrm{D}$ \\
\hline $\mathbf{a}, \mathbf{b}, \mathbf{c 2 . 7}$ & $52312 \mathrm{EN} 45.60 \mathrm{~b}: \mathrm{PL} / \mathrm{krt}[10 \mathrm{mg} / \mathrm{mL}]$ & $0.45 \mu \mathrm{m}$ & $\mathrm{L} / \mathrm{D}$ & $\mathrm{H} / \mathrm{H}$ & $\mathrm{L} / \mathrm{D}$ \\
\hline a, b, c2.8 & PL/krt $[10 \mathrm{mg} / \mathrm{mL}]$ & $0.45 \mu \mathrm{m}$ & $\mathrm{L} / \mathrm{D}$ & $\mathrm{H} / \mathrm{H}$ & $\mathrm{L} / \mathrm{D}$ \\
\hline
\end{tabular}

Table 4.3 Keratinocyte Trial Time Points in Round Two

\begin{tabular}{|c|c|c|c|c|c|}
\hline $\begin{array}{c}\text { well- } \\
\text { set }\end{array}$ & $\begin{array}{c}72 \text { hours post- } \\
\text { passing }\end{array}$ & hour 0 & hour 1 & hour 12 & hour 24 \\
\hline a & $\begin{array}{c}\text { image with optical } \\
\text { microscope \& } \\
\text { digital camera }\end{array}$ & $\begin{array}{c}\text { introduce } \\
\text { test } \\
\text { samples }\end{array}$ & $\begin{array}{c}\text { add L/D \& H/H } \\
\text { stains, image with } \\
\text { confocal }\end{array}$ & $\mathrm{X}$ & $\mathrm{X}$ \\
\hline b & $\begin{array}{c}\text { image with optical } \\
\text { microscope \& } \\
\text { digital camera }\end{array}$ & $\begin{array}{c}\text { introduce } \\
\text { test } \\
\text { samples }\end{array}$ & incubate & $\begin{array}{c}\text { add L/D \& H/H } \\
\text { stains, image with } \\
\text { confocal }\end{array}$ & $\mathrm{X}$ \\
\hline c & $\begin{array}{c}\text { image with optical } \\
\text { microscope \& } \\
\text { digital camera }\end{array}$ & $\begin{array}{c}\text { introduce } \\
\text { test } \\
\text { samples }\end{array}$ & incubate & incubate & $\begin{array}{c}\text { add L/D \& H/H } \\
\text { stains, image with } \\
\text { confocal }\end{array}$ \\
\hline
\end{tabular}




\section{B.8 - Preparation and Application of Histochoice and Hoechst Stain}

The following protocol was acquired and adapted from the Cal Poly BMED 515 Fluorescence Imaging Lab\#1.

\section{Materials:}

- Four-well slides of keratinocyte cells at $60 \%$ or greater confluency

- $\quad$ Phosphate-buffered saline (PBS), $\mathrm{pH} 7.4$

- Histochoice

- Hoechst Stain - Invitrogen 34580 (392/440)

- $15 \mathrm{~mL}$ glass vial

- Aluminum foil

\section{Procedure:}

\section{$\underline{\text { Fixation of Cells }}$}

1. Put on gloves!

2. Aspirate off the existing media. Gently wash cells twice with $1.5 \mathrm{~mL}$ of PBS. Aspirate off PBS after each rinse including the last.

3. Add $1.5 \mathrm{~mL}$ Histochoice to each well. Fix the sample in Histochoice for 30 minutes at room temperature and covered. Prepare the Hoechst stain while incubating the cells in Histochoice.

4. At the conclusion of the 30 minute incubation time aspirate off the Histochoice, rinse twice with PBS, and aspirate off the PBS. 


\section{$\underline{\text { Preparation of Hoechst Stain }}$}

1. Prepare a $2 \mu \mathrm{g} / \mathrm{mL}$ concentration of the Hoechst dye; $1 \mu \mathrm{L}$ of stock solution to $2.5 \mathrm{~mL}$ of PBS. The stock solution is $5 \mathrm{mg} / \mathrm{mL}$. Prepare $400 \mu \mathrm{L}$ of solution for each well. $1 \mu \mathrm{L}$ of stock solution to $2 \mathrm{~mL}$ of PBS.

2. Mix solution in $15 \mathrm{~mL}$ glass vial, securely cap, and vortex. Wrap vial in aluminum foil and store in a dark container until use.

3. Pipette the staining solution on to the surface of each well and incubate covered inside a darkened container for 15 minutes.

4. Remove the stain by gently rinsing the well twice with $1.5 \mathrm{~mL}$ of PBS, aspirate off the PBS each time including the last.

5. When finished, aspirate off all remaining solutions and dispose of the four-well slides in the designated biological waste container.

\section{$\underline{\text { Additional Information }}$}

Image the cells of each well using the confocal microscope filter setting DAPI. If using the DAPI filter in concert with a Qdot filter, set the excitation wavelength of the Qdot filter to 488nm to reduce excitation of the Hoechst stain. 


\section{B.9 - Preparation and Application of Live/Dead Viability/Cytotoxicity Stain}

The following protocol was acquired and adapted from the Cal Poly BMED 515 Fluorescence Imaging Lab\#2.

\section{Materials:}

- Four-well slides of keratinocyte cells at $60 \%$ or greater confluency

- Keratinocyte cell media

- Live Stain: Invitrogen Calcein AM (495/515nm)

- Dead Stain: Invitrogen Ethidium homodimer-1 (EthD-1) (495/635nm)

- $15 \mathrm{~mL}$ glass vial

- Aluminum foil

Procedure:

Prepare Live/Dead Viability/Cytotoxicity Stain

1. Put on gloves!

2. Add $20 \mu \mathrm{L}$ of the supplied $2 \mathrm{mM}$ EthD-1 stock solution to $10 \mathrm{~mL}$ of keratinocyte media in a $15 \mathrm{~mL}$ glass vial. This gives an approximately $4 \mu \mathrm{M}$ EthD-1 solution. Cap and mix the solution thoroughly via vortexing.

3. Combine the reagents by transferring $5 \mu \mathrm{L}$ of the supplied $4 \mathrm{mM}$ Calcein AM stock solution. Cap and mix the solution thoroughly via vortexing. This results in an approximately $2 \mu \mathrm{M}$ Calcein AM and $4 \mu \mathrm{M}$ EthD-1 working solution.

4. Wrap vial in aluminum foil and store in a dark container until use. 


\section{Cell Preparation}

1. Aspirate off the media in each well. Gently wash each well twice with $1.5 \mathrm{~mL}$ of keratinocyte media. Aspirate off the media each time. Add an additional $1.5 \mathrm{~mL}$ of media to each well.

2. Cover each four-well slide until application of the fluorescence stain.

\section{$\underline{\text { Label Cells }}$}

1. Gently add the fluorescence stain to cells. Add $1.5 \mathrm{~mL}$ of Live/Dead working solution to each well of the four-well slide no more than 15 minutes before imaging; this gives a final concentration of $1 \mu \mathrm{M}$ Calcein $\mathrm{AM}$ and $2 \mu \mathrm{M}$ EthD-1.

2. Incubate the cells for 5-15 minutes at room temperature in a covered container protected from light.

3. Image cells using the confocal microscope with filter settings Alexa Fluor488 for Calcein live stain and PI for EthD-1 dead stain.

4. When finished, aspirate off all remaining solutions and dispose of the four-well slides in the designated biological waste container.

\section{$\underline{\text { Additional Information }}$}

The effect of the L/D stain on the cells can be monitored by examining the cells at multiple intervals of time after the introduction of the stain. This temporal monitoring can aid in the determination of the initial cell viability and the influence, if any, of the stain on the viability of the cells. 